\title{
Laboratory Directed Research and Development Program FY 2002
}

March 2003 


\section{Disclaimer}

This document was prepared as an account of work sponsored by the United States Government. While this document is believed to contain correct informution, neither the United States Government nor any agency thereof, nor The Regents of the University of California, nor any of their employees, makes any wartanty, express or implied, or assumes any legill liability or responsibility for the accuracy, completeness, or usefulness of any information, apptratus, product, or process disclosed, or represents that its use would not infringe privately owned rights. Reference herein to any specific commercial produch, process, or service by its trade thame, tradematk, manufacturer, or otherwise, does not necessarily conslitute or imply its endorsement, recombentadation, or favoring by the United States Government or any ngency thereof, or The Regents of the University of California. The views and opinions of atuthors expressed herein do not necessarily state or reflect those of the United States Goverument or any agency thereof or The Regents of the University of California and shall not be used for advertising or product endorsement purposes.

Emest Orlando Lawrence Berkeley National Laboratory

is an equal opportunity employer. 


\author{
Report on \\ Ernest Orlando Lawrence \\ Berkeley National Laboratory
}

\title{
Laboratory Directed \\ Research and Development \\ Program
}

FY 2002

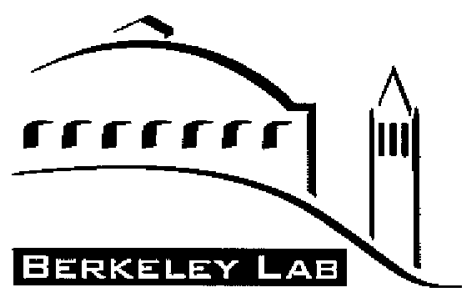

Ernest Orlando Lawrence Berkeley National Laboratory

Berkeley, California 94720

\section{Office of Science}

U.S. Department of Energy 



\section{Table of Contents}

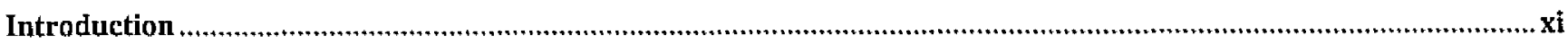

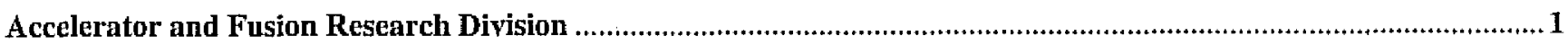

John Corlett

Kem Robinson

Alexinder Zholents

Howard Padmore

Philip Heimann

Robert Schoenlein

Richard Gough

Ian Brown

Eleanor Blakely

Wim Leemans

Jani Reijonen

Ka-Ngo Leung

Gordon Wozniak

Rick Firestone

Eleanor Blakely

Steve Kahl (UCSF)

Jasmina Vujic (UC Berkeley)

Phillip Colella

Eric Esarey

Alex Friedman

Robert Ryne

Jean-Luc Vay

Thomas Schenkel

Jeffrey Bokor

Wiltiam Turner

Max Zolotorev

Robert Schoenlein

Alexander Zholents
An Ultra-fast X-ray Soutce for Femtosecond Dynamics: Feasibility and Preconceptual Design

Large, Patterned Networks of Living Neurons 3

Novel Coherent Terahertz and Infared Source Using a Iaser Wakefield Accelerator and Applications 4

Compact Coaxial Deuterium-Deuterium (D-D) Neutron Generator and Moderator Research .5

Advanced Simulation of Complex Beam Systems 7

Solid State Quantum Computer Development with Single Ion Implantation .....9

Synchrotron and Wiggler Radiation Measurement of the Longitudinal Bunch

Distribution in Hadron Colliders.

A Source of Far Infrared Radiation Based on a Material with an Optically Switched Permittivity

Advanced Light Source Division.

M. Zahid Hasan Jonathan Denlinger James Underwood

Zhi-xun Shen

Zahid Hussain
Filling in a "Big Gap": A Novel Momentum-Resolved Inelastic Emission Soft X-Ray Spectrometer for the Study of Strongly Correlated Quantum Systems 
Michael Martin

John Byrd

Wayne McKinney

David Robin

Alexander Zholents

Robert Schoenlein

Howard Padmore

Paul Alivisatos

Raymond Jeanloz

Simon Clark

Howard Padmore

Garrison Sposito

Alain Manceau (CNRS,

U. Grenoble)

Shaul Mukamel

(U. Rochester)

Robert Schoenlein

Thornton Glover

Andreas Scholl

Aaron Lindenberg

Howard Padmore

Joachim Stöhr

Michel Van Hove

Andrew Canning

Lin-Wang Wang
Development of a Far-IR Synchrotron Radiation Source at the ALS

14

High-Pressure Research at the Advanced Light Source .16

Spatially Resolved X-ray Absorption and Diffraction Applied to Problems in Environmental Science

Simulations of Femtosecond X-ray Spectra of Photoexcited Molecules .... 19

Picosecond (P-sec) Time-Resolved Photo-Electron Emission Microscopy on Magnetic Nano-Structures 20

Bonding in Low-Dimensional Structures: Theory and Computation. 21

Chemical Sciences Division.

Corwin Booth

Harvey Gould

Hiroshi Nishimura

Stephen Leone

Daniel Neumark

Kenneth Raymond and Robert Bergman

David Shuh
Disorder and Multiple Length Scales in Non-Fermi Liquid f-electron Intermetallics.

Development of a Neutral Molecule Synchrotron Storage Ring

Coherent Control and Quantum Information in Polyatomic Molecules...........26

Photoionization and Photoelectron Spectroscopy of He Droplets.

Development of Highly Selective Catalysts in Supramolecular Nanoscale Reaction Vessels.

Scientific Investigations and Technique Development of Wet Spectroscopy, High Pressure Photoelectron Spectroscopy, and STXM for Molecular Environmental Science 


\section{Computing Sciences}

(National Energy Research Scientific Computing Center, Mathematics Department, Computational Research

Division and Information Technologies and Services Division)

David H. Bailey

Xiaye Li

Grigory Barenblatt

E. Wes Bethel

Silvia Crivelli

Oliver Kreylos

Nelson Max

Bernd Hamann

Chris Ding

Joseph Grcar

Paul Hargrove

Niels Jensen

Xiaoye Li

Leonid Oliker

Katherine Yelick

Ravi Malladi

Juan Meza

Gregory Milter

Ekow Otoo

Ali Pinar

William McCurdy

Thomas Rescigno
The Experimental Mathematician's Toolkit.

31

Nonlinear Mathematical Models of Phenomena Related to Petroleum, Mining,

and Geological Engineering

Infrastructure for Inproving Protein Structure Prediction in Computational

Biology.

New Machine Learning and Data Mining Methods for Genomics and Information

Retrieval 35

Numerical Simulation of Fuel Cells 37

High Performance System Area Networking for PC Clusters. .37

Atomic Scale Modeling of Materials Chemistry .38

Suitability of Alternative Architectures for Scientific Computing in the NERSC-5 Time Frame.

Segmentation of Mammary Gland Ductal Structure using Geometric Methods......

Parallel Methods for Robust Optimization and Uncertainty Quantification.....41

Second-order Methods for Solid-Fluid Shock Coupling with Application to Martian Meteorites.

Adaptive File Replication and Coordinated Transfer for Data Intensive Grid

Applications

Combinatorial Algorithms in Scientific Computing

Scalable Methods for Studying Collisional Breakup and Rearrangement

Processes.

Earth Sciences Division

Jillian Banfield

Microbial Controls on Metals in the Environment......

Kristie Boering

Atmospheric Chemistry and Climate on Extrasolar Bodies: Experimental Constraints for Predictions of Habitability and Spectroscopic Signatures of Life 
Jeffrey Boore

Brent Mishler

Carrine Blank

Terry Hazen

Curtis Oldenburg

Sharon Borglin

Michael Manga

Curtis Oldenburg

William Riley

Margaret Torn

Marc Fischer

John Harte

Helen $\mathrm{He}$

Glenn Waychunas

Jillian Banfield

Hoi-Ying Holman

Paul Alivisatos
The Complete Genome Sequences of Hyperthermophilic Bacteria Can Tell Us

About the Nature of the Early Earth's Environment

Aerobic Bioremediation of Landfills

Coupling of Seismologic and Hydrologic Processes

Development of Monitoring Strategies for Carbon Sequestration Verification

Using Coupled Subsurface and Subaerial Simulation

. .56

Applying a Coupled Climate-Land Surface Regional Model to Deduce Trends in

Soil Moisture from Air Temperature Data .....

.58

Reactivity of Nanoparticles in Natural Environments

.59

\section{Engineering Division}

Henry Benner

Fast Lipoprotein Identification Process

.63

Ronald Krauss

Microprojectile Accelerutor for Targeted Biological Transformation

.65

Abby Dernburg

Environmental Energy Technologies Division

Hashem Akbari

Paul Berdahl

Michael Apte

Lara Gundel

Anthony Hansen

Joeseph Eto

Chris Marnay

William Fisk

Regine Goth-Goldstein

Mark Mendell
Development of Cool Colored Shingles

.67

Miniaturized Systems for Particle Exposure Assessment ...................................68

Simulation and Interpretation of the Effects of Transmission Congestion on Restructured Wholesale Electricity Markets

Indoor Bioaerosol Detection and Quantification by Polymerase Chain Reaction (PCR)

Health Effects of Indoor and Outdoor Particle Concentrations, Assessed with Epidemiology .73 
Mary Helen Barcellos-Hoff

Damir Sudar

Bahram Parvin

Abby Dernburg

Daniel Callahan

Richard Schwartz

Anat Biegon

Mark Biggin

Abby Dernburg

Michael Eisen

Bing Jap

Carolyn Larabell

Saira Mian
Quantitative Spatial and Temporal Resolution of Multicellular Interactions ...75

Positron Emission Tomography (PET) Ligands for the N-methyl-D-aspartate (NMDA) Receptor Channel 76

A High Sensitivity $I n-V i v o$ Crosslinking Method............................................77

Dynamic Reorganization of Chromosome Architecture during Meiosis...........78

Experimental and Computational Analysis of the Mechanisms and Logic of Eukaryotic Transcriptional Regulation... 79

Structural Studies of Presenilin-1, a Membrane Protein Critical to the Onset of Alzheimer's Disease. 82

Tracking Proteins in Light and Soft X-Ray Microscopy .83

Systems Biology: Biological Input-output Devices .84

Materials Sciences Division

A. Paul Alivisatos

Inorganic Liquid Crystals. .87

Peidong Yang

Jean Frechet

Carolyn Bertozzi

Daniel Chemla

Leonid Butov

J. C. Séamus Davis

Dung-Hai Lee

Joseph Orenstein

Oscar Dubón, Jr.

Charles Fadley

Malcolm Howells

Matthew Francis

D. Frank Ogletree Miquel Salmeron

Zi Qiang Qiu
Functional Bone-Like Materials: A Biomimetic Synthetic Approach . .87

Condensation of Indirect Excitons in Coupled Quantum Well

Nanostructures.

Nanoscale Electronic Phase Separation: A New Paradigm for Complex

Impurity Overlayers in Semiconductor Epitaxy: Expanding the T_imits of Alloy Composition and Nanostructure Synthesis

Holographic Imaging with X-rays: Fluorescence and Fourier Transform Holography

Self-Assembling Artays of Nanocrystals Templated by Cytoskeletal Proteins

Investigation of High-pressure Surface Reactions with a Molecular Beam of Reaction Products .97

Photoemission Study of Magnetic Quantum Well Interaction . .99 Electronic Materials. 
Nuclear Science Division

Paulo Bedaque

I-Yang Lee

Kevin Lesko

Howard Wieman

Fred Bieser

Stuart Kleinfelder

Howard Matis
Effective Field Theory and Few-Nucleon Systems

Design of Digital Signal Processing Electronics for High-Resolution Radiation Detectors. 104

Detector Research and Development for Low Energy Solar Neutrino Detectors. 105

Research on a Next Generation Vertex Detector 107

Physical Biosciences Division.

Jay Groves

Arup Chakraborty

Thomas Earnest

Randy Moon (HHMI,

U. of Washington)

Robert Glaeser Kenneth Downing

Eva Nogales

Esmond $\mathrm{Ng}$

Ravi Malladi

Teresa Head-Gordon

John Kuriyan

Michael Marletta

Gerry McDermott

Faw Yang
Cooperative Effects Determining Fidelity in Cellular Recognition .109

Molecular Recognition and Protein/Protein Interactions in Signal

Transduction

Teraflop Challenges in Single-Particle Electron Crystatlography 112

Novel Synchrotron Experiments to Determine Hydration Forces for Molten Globules and Model Proteins for Extremophiles.

Allosteric Mechanisms in Proteins Involved in Cell Signaling.

Orphan Guanylate Cylase Receptors in C. Elegants.

Development of Techniques for Structural Analysis of Large, Multi-Subunit Eukaryotic Transcription Complexes .116

Conformation and Reaction Dynamics at the Single-Molecule Level 117

Physics Division

Ian Hinchliffe Murdock Gilchriese

Modeling of High Energy Physics Detectors

Adrian Lee

Helmuth Spieler
POLARBEAR: An Experiment to Measure Polarization Anisotropy in the Cosmic Microwave Background 
Michael Levi

Saul Perlmutter

Greg Aldering
Foundations for a SuperNova/Acceleration Probe (SNAP) 121

Sub-Project 00026: Development of an Advanced Imaging Array ., 121 Sub-Project 00027: Nearby Supernova Search with Ten-Fold Increase in Efficiency.

Cross Divisional

Andrew Canning

Steven Louie

Charles Harris

John Arnold

John Kerr

Stephen Johnson
Computational Methods for Electronic Structure Codes in Materials Science (Ground-State and Excited-State Properties)

Investigation of Charge Transfer in Organic Electronics using Ultrafast Spectroscopy and Targeted Synthesis 126 



\section{Introduction}

The Ernest Orlando Lawrence Berkeley National Laboratory (Berkeley Lab or LBNL) is a multi-program national research facility operated by the University of California for the Department of Energy (DOE). As an integral element of DOE's National Laboratory System, Berkeley Lab supports DOE's missions in fundamental science, energy resources, and environmentitl quality. Berkeley Lab programs advance four distinct goals for DOE and the nation:

- To perform leading multidisciplinary research in the computing sciences, physical sciences, energy sciences, biosciences, and general sciences in a manner that ensures employee and public safety and protection of the environment.

- To develop and operate unique national experimental facilities for qualified investigators.

- To educate and train future generations of scientists and engineers to promote national science and education goals.

- To transfer knowledge and technological innovations and to foster productive relationships among Berkeley Lab's research programs, universities, and industry in order to promote national economic competitiveness.

Berkeley Lab's research, in general, and the LDRD program, specifically, all unclassified, support DOE's important science mission, as articulated in the Secretary of Energy's FY 2003 Budget Rollout. This statement includes that "we... want to use the talents nurtured by our science program to leapfrog today's energy security problems," and that "as the irreplaceable foundation for tomorrow's security demands, we need a strong physical science program." Furthermore, "DOE's program in science is a story of immense accomplishments and vast promise...We are leaders in pursuit of the basic understanding of matter and physical science." In addressing fundamental research, the statement further notes that "our work to better understand the fundamentals of energy production and its impacts has had far-reaching applications in biology, chemistry, nuclear medicine, and materials science." The LDRD program supports these goals; and some of the research referenced in the Secretary's statement has its origins in the LDRD program.

The Berkeley Lab Laboratory Directed Research and Development Program FY 2002 report is compiled from annual reports submitted by principal investigators following the close of the fiscal year. This report describes the supported projects and summarizes their accomplishments. It constitutes a part of the Laboratory Directed Research and Development (LDRD) program planning and documentation process that includes an annual planning cycle, project selection, implementation, and review.

The Berkeley Lab LDRD program is a critical tool for directing the Laboratory's forefront scientific research capabilities toward vital, excellent, and emerging scientific challenges. The program provides the resources for Berkeley Lab scientists to make rapid and significant contributions to critical national science and technology problems. The LDRD program also advances Berkeley Lab's core competencies, foundations, and scientific capability, and permits exploration of exciting new opportunities. All projects are work in forefront areas of science and technology. Areas eligible for support include the following:

- Advanced study of hypotheses, concepts, or innovative approaches to scientific or technical problems;

- Experiments and analyses directed toward "proof of principle" or early determination of the utility of new scientific ideas, technical concepts, or devices; and

- Conception and preliminary technical analyses of experimental facilities or devices.

The LDRD program supports Berkeley Lab's mission in many ways. First, because LDRD funds can be allocated within a relatively short time frame, Berkeley Lab researchers can support the mission of the Department of Energy (DOE) and serve the needs of the ration by quickly responding to forefront scientific problems. Second, LDRD enables Berkeley Lab to attract and retain highly qualified scientists and supports their efforts to carry out worldleading research. In addition, the LDRD program also supports new projects that involve graduate students and postdoctoral fellows, thus contributing to the education mission of Berkeley Lab.

Berkeley Lab has a formal process for allocating funds for the LDRD program. The process relies on individual scientific investigators and the scientific leadership of Berkeley Lab to identify opportunities that will contribute to scientific and institutional goals. The process is also designed to maintain compliance with DOE Orders, in particular DOE Orders 413.2 dated March 5, 1997, and 413.2A, dated January 8, 2001. From year to year, the distribution of funds among the scientific program areas 
changes. This flexibility optimizes Berkeley Lab's ability to respond to opportunities.

Berkeley Lab LDRD policy and program decisions are the responsibility of the Laboratory Director. The Director has assigned general programmatic oversight responsibility to the Deputy Director. Administration and reporting on the LDRD program is supported by the Directorate's Office for Planning and Strategic Development. LDRD accounting procedures and ftnancial management are consistent with the Laboratory's accounting principles and stipulations under the contract between the University of California and the Department of Energy, with accounting maintained through the Laboratory's Chief Financial Officer.

In Fiscal Year (FY) 2002, Berkeley Lab was authorized by DOE to establish a funding ceiling for the LDRD program of $\$ 14.5 \mathrm{M}$, which equates to about $3.2 \%$ of Berkeley Lab's FY02 projected operating and capital equipment budgets. This funding level was provided to develop new scientific ideas and opportunities and allow the Berkeley Lab Director an opportunity to initiate new directions. Budget constraints limited available resources, however, so only $\$ 11.9 \mathrm{M}$ was expended for operating and $\$ 0.5 \mathrm{M}$ for capital equipment ( $2.6 \%$ of actual Berkeley Lab FYO2 costs).

In FY02, scientists submitted 152 proposals, requesting over $\$ 24.1 \mathrm{M}$ in operating funding. Eighty-six. projects were funded, with awards ranging from $\$ 15 \mathrm{~K}$ to $\$ 1200 \mathrm{~K}$. 


\section{Accelerator and Fusion Research Division}

\section{An Ultra-fast X-ray Source for Femtosecond Dynamics: Feasibility and Pre-conceptual Design}

Principal Investigators: John Corlett, Kem Robinson, Alexander Zholents, Howard Padmore, Philip Heimann, and Robert Schoenlein

Project No.: 01003

\section{Project Description}

Ultra-short pulses of visible light produced by fsec laser techniques have revolutionized many areas of physical, chemical and biological science. These techniques are however limited in not being able to directly probe atomic motion. X-ray techniques though, through $\mathrm{x}$-ray diffraction and spectroscopy are capable of exquisite sensitivity to atomic motion. Combining $x$-ray techniques with short pulses is being pioneered at the Advanced Light Source (ALS), but it is clear alreaty that although of huge potential, we need to be planning for a much more intense source to attack the most exciting areas of science.

We have identified a method of producing ultra-short x-ray pulses with an intensity of up to $10^{7}$ photons per pulse, $0.1 \%$ bandwidth, at $10 \mathrm{keV}-10^{6}$ times higher than currently used at the ALS; use of this source would revolutionize the study of ultra-fast dynamics across a broad range of scientific areas.

The purpose of this proposal is therefore to develop critical aspects of the proposed technique in continuation of the prior year's work.

The design of a new type of $x$-ray light source, based on a superconducting linear accelerator, will be intensively studied. The machine will produce very short, intense x-ray pulses from an electron beam accelerated from a photocathode gun through a linear accelerator, the bunches will then be compressed, and then rotated to enable further optical compression to a bunch length of 50 femtoseconds (fs) full width at half maximum (FWHM). X-rays will be generated by wiggler or undulator magnets, as in the ALS. This project will look at pre-conceptual design and R\&D on critical components and systems. Initial work has verified the tremendous scientific potential of a national facility, and the practicality of all aspects of the project.

\section{Accomplishments}

We have developed design concepts for a novel synchrotron radiation facility for $\mathrm{X}$-ray studies of ultrafast dynamic processes. A recirculating superconducting linac is used for acceleration of electrons produced by a highbrightness photocathode radio frequency (RF) gun, at a bunch repetition rate of approximately $10 \mathrm{kFz}$. Electrons emitted from the source are carefully manipulated in tailored magnetic fields to produce a small vertical emittance, which is key to producing the -60 femtosecond $\mathrm{x}$-ray pulses from the electron pulses of $-2 \mathrm{ps}$ duration. After acceleration by four passes through a superconducting recirculating accelerator, a specialized technique is employed to produce the ferntosecond $\mathrm{x}$-ray pulses by first imprinting on the electron bunches a transverse momentum in the direction of the small emittance that is correlated to longitudinal position within the bunch. This action results in an emitted $x$-ray pulse with a time-correlated spatial and angular distribution. The correlated $x$-ray pulse is then compressed by crystal optics to achieve the ultra-fast photon pulse.

Our approach for producing a short $x$-ray pulse relies on the availability of an electron beam source with a flat aspect ratio so that one transverse dimension ( $y$, vertical) is small. This principle has been successfully demonstrated at the Fermilab/NICADD Photoinjector Laboratory (FNPL). An $\mathrm{x} / \mathrm{y}$ emittance ratio of order $10^{2}$ may be achieved by this procedure, with a normalized enittance of $\gamma\left(\varepsilon_{x} \times \varepsilon_{y}\right)^{0.5} \approx 10^{-6} \mathrm{~m}-\mathrm{rad} / \mathrm{nC}$ at the cathode. The successful demonstration at the FNPL of flat beams with emittance ratio 50:1 is highly encouraging and we have become major participants in the experimental and computational studies collaboration to help further work in this regard.

Conventionally, photocathode RF guns employ a halflength pillbox cell for the cathode cavity followed by a full cell for rapid acceleration of emitted electrons. For continuous wave (CW) or high duty factor operation, thermal limitations may prevent such a design from operating at sufficiently high gradient. We have produced a conceptual design with optimized cavity geometry to allow cooling of the cavity surfaces, and operation at high gradient and high repetition rate. In this design the first cell is modified by the inclusion of a re-entrant nose-cone, on the end face of which the photo-cathode is mounted. This nose-cone serves two purposes: it increases surface area to reduce deposited power density, and it enhances the accelerating electric field at the cathode. 
Our linac design is based on superconducting RF technology developed for the TESLA project. Our design considerations here are for a peak accelerating gradient of $20 \mathrm{MV} / \mathrm{m}$. Identical cryomodules are used for the main linac and the injector linac. In addition to the $1.3 \mathrm{GHz}$ linacs, we require $3.9 \mathrm{GHz}$ superconducting cavities to provide the deflecting voltage along the bunch, and third harmonic cavities to linearize the longitudinal phase space in the injector. We have developed concepts for $\mathrm{CW}$ operation of these demanding RF systems.

Synchronization and timing of the $-60 \mathrm{fs} x$-ray pulse to the optical pulse of the experimental lasers is important in studies of ultra-fast dynamics. For our scheme we have found that this timing jitter is dominated by the phase jitter of the deflecting cavities with respect to the experimental laser pulse. We have begun to develop a scheme for timing signal distribution and phase and amplitude control of the superconducting cavities that is expected to provide $\mathrm{x}$-ray pulse to laser pulse stability of order \pm 50 femtoseconds.

Emittance control and understanding and mitigation of collective effects is critical to a successful machine design, and we have addressed key aspects of accelerator physics involved in beam break-up, coherent synchrotron radiation, the influence of resistive wall wakefields, and other effects. For example, with typical misalignment errors of $0.5 \mathrm{~mm}$ for individual cavities and $0.25 \mathrm{~mm}$ for cryomodules, simulation results show that by controlling the betatron phase advance between passes through the linac, and the initial offset of the beam, the resilting projected emittance growth from cavity wakefields is only a few percent.

Longitudinal dynamics have been modeled from the RF gun through all passes of the linac, and emittance dilution due to non-linearities in the RF waveform have been assessed. In the injector, we propose to use harmonic cavities to linearize the longitudinal phase-space. An energy spread of $\pm 200 \mathrm{keV}$ in the final arc requires a bunch of a less than few piscoseconds length.

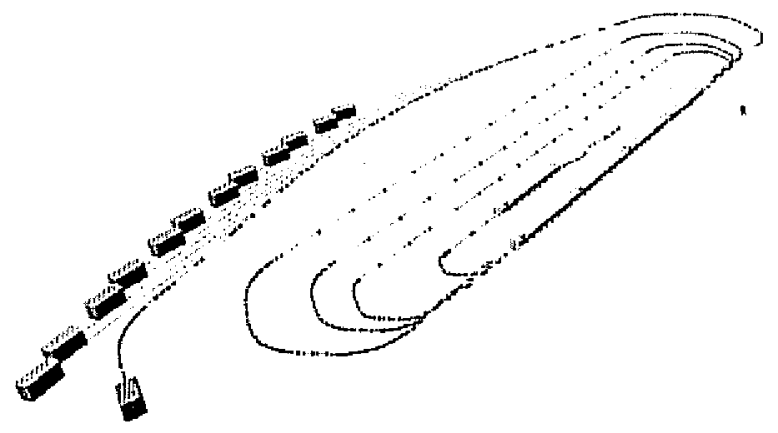

Figure 1: Machine layout of a facility for production of ultra-fast hard $x$-ray pulses, based on a recirulating superconducting linac. The proposed facility would provide $x$-ray pulses with time structure optimized for study of dynamic processes occuring within the fundamental timescale of atomic motion. A high-brighthess electron beam generated in a radiofrequency photocathode gun is manipulated in a combination of solenoidal and quadrupole magnetic fields to produce a large $x / y$ enittance ratio. This specially formed beam is then accelerated in the injector linac, transported to the main linac, and guided through four passses of the main linac and transport arcs. In the final pass, the bean reaches an energy of $2.5-3.0 \mathrm{GeV}$. On entering the final arc, the hightbrightness beam is further manipulated in deflecting cavities, such that the $x$-ray beams emitted in undulators may be conpressed in optical systems to time durations of less than 100 femtoseconds. The machine footprint is approximately $150 \times 50 \mathrm{~m}$, and provides multiple beamlites. After radiating in the photon production section (the final arc), the beam is absorbed in a shielded beam dtump.

\section{Publications}

A. Zholents, I. Reichel, et al., "Initial Lattice Studies for the Berkeley Femtoseçond X-ray Light Source," European Particle Accelerator Conference, Paris, June 2002, Submitted for publication.

http://accelconf.web.cern.ch/AccelConf/e02/

S. De Santis, and A. Zholents, "Beam Break-Up analysis for the Berkeley Femtosource," European Particle Accelerator Conference, Paris, June 2002, Submitted for publication. http://accelconf.web.cern.ch/AccelConffe02/

R.A. Rimmer, N. Hartman, S. Lidia, and S.H. Wang, "A High-Gradient High Duty-Factor RF Photocathode Electron Gun," European Particle Accelerator Conference, Paris, June 2002, submitted for publication. http://accelconf.wel.cern.ch/AccelConfleO2/

D. Li, and J.N. Corlett, "RF Deflecting Cavity Design for the Berkeley UItrafast X-ray Source," European Particle Accelerator Conference, Paris, June 2002, Submitted for publication. http://accelconf.web.cern.ch/AccelConf/e02/

J.N. Corlett, W.C. Barry, J.M. Byrd, R. Schoenlein, and A. Zholents, "Synchronization of X-ray Pulses to the Pump Laser in an Ultrafast X-ray Facility," European Particle Accelerator Conference, Paris, June 2002, Submitted for publication. hittp://accelconf.web.cem.ch/AccelConffe02/

J.N. Corlett, W.C. Barry, et al., "A Recirculating Linac Based Synchrotron Light Source for Ultrafast X-ray Science," European Particle Accelerator Conference, Paris, June 2002, Submitted for publication. http://accelconf.web.cern.ch/AccelConfle02/

E. Thrane, C. Bohn, et al., "Photoinjector Production of a Flat Electron Beam," Linac 2002, Korea, August 2002, Submitted, http://linac2002.postech.ac. $\mathrm{k} /$ 
J.N. Corlett, S. Lidia, R. Schoenlein, and A. Zholents, "An RF Photocathode for a Recirculating Linac-based UItrafast Dynamics Facility," Workshop on Laser Issues for Electron RF Photoinjectors, SLAC, October 2002, Submitted for publication. http://www-conf.slac.stanford.edu/li-erp/

D. Li, and J. Corlett, "Preliminary Coupler Design for a Seven-Cell SC Deflecting Cavity," Workshop on HighPower Couplers for Superconducting Accelerators, Jefferson Lab, October 2002, in preparation for submission. http://wwww.jlab.org/hpc2002/

J.N. Corlett, W. Barry, et al., "Feasibility Study for a Recirculating Linac-Based Facility for Femtosecond Dynamics," LBNL publication, December 2002, In preparation for submission.

\section{Large, Patterned Networks of Living Neurons}

Principal Investigators: Richard Gough, lan Brown and Eleanor Blakely

Project No.: 01001

\section{Project Description}

While techniques such as magnetic resonance imaging and positron emission tomography have provided insight into the location of neural activity within the brain, the minimum resolvable volume $\left(\sim 1 \mathrm{~mm}^{3}\right)$ contains $-10^{3}-10^{4}$ neurons. In order to learn the details of how large systems of neurons communicate, we need first to develop methods for growing networks of large numbers of live neurons whose dendrite and axon connections can be controlled in pre-determined ways, and second to develop means for stimulating and monitoring excitation. Success with this challenge will be of great importance to our understanding of the working of the human brain and peripheral nervous system, and to novel kinds of computer architecture. Large in-vitro networks could show, for example, the emergence of stable patterns of activity, and could lead to an understanding of how groups of neurons learn after repeated stimulation.

Our approatch to patterning was selective plasma deposition on the substrate on which the neural array was subsequently grown, using ion species that enhance or inhibit neural growth. We have explored growth-stimulating and growthinhibiting surface modification methods so as to learn the most effective method (highest "contrast"). Lithographic masks of various feature sizes and network patterns were used to form the arrays.

\section{Accomplishments}

The goal of the work was to discover and develop plasmabased methods for selectively enhancing or inhibiting neuron growth, both by energetic ion implantation and by plasma deposition, and then to use these methods to form large patterned arrays of live neurons on suitable substrates. We have demonstrated that high energy metal ion implantation and also low energy plasma deposition of ultra thin films both provide the kind of tool we were seeking.

Neuron cultures were grown, monitored, and quantified on substrates that had been ion implanted or plasma deposited using a range of plasma / ion beam parameters, particularly variation of the ion species. There are substantial differences in the effects of these methods on neuron growth due to different ion species - this approach provides a valid approach for altering the neuro-compatibility of the substrate on which the neural culture is to be grown. We determined that the best plasma processing, in terms of enhancing selective neuron growth, was the formation of a super-thin (of order 100 angstrom) layer of diamond-like carbon (DLC) atomically mixed into the substrate surface using filtered cathodic arc plasma deposition. Some test patterns of diamond-like carbon were made and we were able to subsequently grow neurons (PC-12 rat neurons) on the pattern. The neuron growth "patterning contrast ratio" (ratio of neuron density on the plasma-treated regions to neuron density on the untreated regions) was very high, with prolific growth on the plasma-treated regions and virtually none on the untreated regions.

Figure 2 below provides an example of the application of the technique for neural array patterning. Neuron growth was on a glass substrate processed by plasma deposition of -150 angstrom diamond-like carbon film. Prior to deposition, "LBNL" was written on the glass slide using a fine marker pen, and then the DLC was deposited. After DLC deposition, the ink was removed with alcohol, thus lenving "LBNL" patterned in negative in the DLC film. The slide was then coated with Type I Collagen and seeded with PC-12 rat neurons. The neurons were allowed to grow for 3 days, at which point Nerve Growth Factor (NGF) was added. The micrographs shown in the micrograph were taken after a growth period of 6 days after initiation of the cultures.

This work has demonstrated the suitability of filteredvacuum-arc plasmes deposition of diamond-like carbon for forming patterned arrays of large numbers of live neurons. The energetic plasma deposition process provides a means for selective neuron attachment, growth, and differentiation on the surface. The neuron growth contrast ratio (ratio of neuron density on plasma-treated regions to neuron density 
on untreated regions) can be very high, adequate for the fabrication of large patterned arrays of neurons.

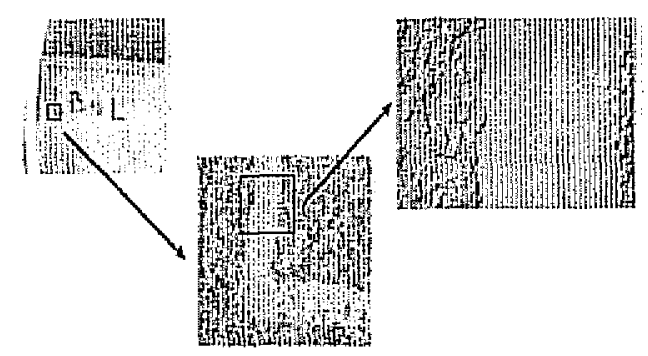

Figure 2: Patterned growth of neurons to form "LBNL" A diamond-like carbon thin film with " $L B N L$ " patterned into it (in negative) was plasma deposited on a glass slide. The slide was then coated with collagen and seeded with PC-12 rat neurons. The micrograph shown was taken after a growth period of 6 days after initiation of the ctilture

\section{Publications}

I.G. Brown, K.A. Bjornstad, E.A. Blakely, J.E. Galvin, O.R. Monteiro and S. Sangyuenyongpipat, "Surface Modification for Large Patterned Arrays of Neurons using Plasma Methods," Invited Paper, $3^{\text {rd }}$ International Conference on Surface Engineering, Chengdu, China, Oct 10-13, 2002.

I.G. Brown, K.A. Bjornstad, E.A. Blakely, J.E. Galvin, O.R. Monteiro and S. Sangyuenyongpipat, "Growth of Large Patterned Arrays of Neurons using Plasma Methods," Invited Paper, $11^{\text {tht }}$ International Congress on Plasma Physics, (ICPP'02), Sydney, Australia, July 15-19, 2002. To be published in Plasma Physics and Controlled Fusion, in press.

I.G. Brown, O.R. Monteiro, E.A. Blakely, K.A. Bjornstad, J.E. Galvin and S. Sangyuenyongpipat, "Applications of Vacuum Arc Plasmas to Neuroscience," NATO Advanced Research Workshop on Emerging Applications of VacuumArc-Produced Plasma, Ion and Electron Beams, Lake Baikal, Russia, June 24-27, 2002, proceedings in press.

E.A. Blakely, K.A. Bjornstad, J.E. Galvin, O.R. Monteiro, I.G. Brown and S. Sangyuenyongpipat, "Selective Neuron Growth on Ion Implanted and Plasma Deposited Surfaces," 29th IEEE International Conference on Plasma Science (ICOPS'02), May 26-30, 2002, Banff, Alberta, Canada. IEEE Conference Record, Cat. No. 02CH37340, p. 253.

\section{Novel Coherent Terahertz and Infared Source Using a Laser Wakefield Accelerator and Applications}

\author{
Principal Investigators: Wim Leemans \\ Project No.: 02001
}

\section{Project Description}

This proposal is aimed at (1) the development of a source of high peak flux, coherent, ultra-short pulse ("single" cycle) infrared (IR) and terahertz (THz) radiation from a laser-driven accelerator and (2) investigating the interaction of this radiation with atomic media (which includes gaseous and condensed media.) Source development will comprise the design and implementation of the THz radiator, collection optics and diagnostics, calibration of figures of merit (flux, temporal, spatial and spectral properties), and systematic characterization of the source response to electron beam and laser parameters. Detailed theoretical calculations in support of these measurements will be performed. Coherent $\mathrm{THz}$ pulses with peak source strengths several orders of magnitude beyond current technology should be obtainable, thus allowing for applications to be performed in new nonlinear regimes. Practical application of the THz source will be demonstrated through nonlinear and time-resolved experiments such as high field transport in semiconductors.

\section{Accomplishments}

In our experiments, electron bunches were generated by focusing laser pulses from a Ti:Al2O3 laser operating at $800 \mathrm{~nm}$ onto a supersonic Helium gas jet with a length of 2 $\mathrm{mm}$, mounted inside an evacuated vacuum chamber. The laser pulses were focused, and the total charge per bunch and spatial profile of the electron beam were measured using an integrating current transformer (ICT) and phosphor screen imaged onto a 16 bit CCD camera, respectively.

Far-infrared measurements were carried out at several different frequencies. The electron beam charge per bunch was varied by changing either the laser pulse duration or, for fixed laser pulse duration, by changing the position of the gas jet with respect to the laser beam focal position. Quadratic dependence of the peak amplitude of the $94 \mathrm{GHz}$ microwave detector vs. bunch charge was observed, consistent with coherent emission of radiation. 
A Si-based bolometer (manufacturer Infrared Laboratories) cooled at $4.2 \mathrm{~K}$ with a $100 \mathrm{~cm}-1$ cut-on filter is now being used to measure radiation in the $0.015-1 \mathrm{~mm}$ range. Quadratic dependence of the bolometer signal with charge per bunch has been observed. We are currently in the process of quantifying the amount of radiated energy per pulse, within the collection solid angle as well as measuring the spectrum.

In parallel, we have also started the implementation of an electro-optically sampled system where the far-infrared radiation (FIR) from the electron beam is made to propagate collinearly with a 50 fs laser pulse through a nonlinear crystal. The birefringence induced in the crystal by the FIR causes the polarization of the incident laser beam to rotate. Using an analyzing polarizor then enables measuring the shape of the electric field of the FIR. This method will be implemented on the laser accelerator during FY03 and will enable us to characterize the puIse shape of the radiation.

Theoretical modeling of the expected performance has been carried out as well. Based on analytical calculations, an optimized source has the potential to exceed the energy per pulse produced by conventional sources by nearly three orders of magnitude enabling a new regime for experiments relying on excitation of physical processes with a laser and probing them with FIR. In parallel, particle-in-cell simulations ate being carried out to aid in optimizing the source performance.

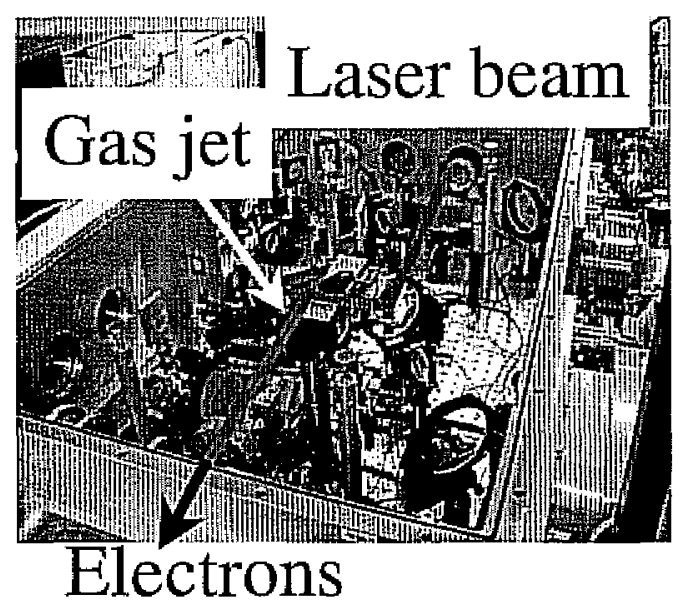

Figure 3: Photograph of the inside of the vacuum chamber housing the laser driven accelerator. The main drive laser beam is focused above a gas jet where the electrons are born through ionization and subsequently accelerated in the wakefield excited by the intense laser beam.

\section{Publications}

W.P. Leemans et al., "Generation of THz Radiation Using Laser Accelerated Electron Bunches," Science, in preparation.

B. Marcelis et al., "Coherent Radiation in the $\mathrm{GHz}$ and $\mathrm{mm}$ wave Regime from Laser Wakefield Accelerated Ultrashort Electron Bunches," Advanced Accelerator Concept Workshop 2002, Oxnard CA, June 25, 2002.

B. Marcelis et al., "Coherent Radiation in the $\mathrm{GHz}$ and mmwave Regime from Laser Wakefield Accelerated Ultrashort Electron Bunches," Annual Meeting of the American Physical Society-Division of Plasma Physics 2002, Orlando FL, November I I-15, Bull. Am. Phys. Society.

J. van Tilborg et al., "Coherent Radiation in the $\mathrm{GHz}$ and mm-wave Regime from Laser Wakefield Accelerated Ultrashort Electron Bunches," Particle Accelerator Conference 2003, May 2003, in preparation.

\section{Compact Coaxial Deuterium- Deuterium (D-D) Neutron Generator and Moderator Research}

Principal Investigators: Jani Reijonen, Ka-Ngo Leung, Gordon Wozniak, Rick Firestone, Eleanor Blakely, Steve Kahl (UCSF) and Jasmina Vujic (UC Berkeley)

Project No.: 00002

\section{Project Description}

Several areas of basic research and applied neutron research require an intense source that is compact, inexpensive, and safe to operate. The current options for neutron sources are reactors and high-energy accelerators. The Plasma and Ion Source Technology group of the Accelerator and Fusion Research Division has developed a unique technology that provides a neutron tube producing intense neutron flux.

For the neutron tube testing and development a test capability was needed. This same capability could be used for various research activities, like neutron induced Prompt Gamma Activation Analysis (PGAA) and various studies involving ${ }^{10} \mathrm{~B}$ delivery agents for the Boron Neutron Capture Therapy (BNCT). 


\section{Accomplishments}

During FY02 three major aspects of the test capability were developed and fabricated, namely the high flux, coaxial neutron generator, moderator/shielding structure and the equipment necessary to run the generator, HV shielding, yacuum systems and radiation safety monitoring systems. The experiments consisting of PGAA and cell radiations will be also discussed.

\section{Neutron Generator}

The previously tested and developed novel concept of coaxial neutron generator was utilized here. The co-axial neutron generator consists of internal $\mathrm{RF}$-driven plasma generator (ion source) and co-axially placed cylinder-shape target assembly. This design provides enough target area for high power (high yield) operation of the generator, still in relatively compact outer dimensions. The size of the generator is approximately 14" height and 12 " in diameter. The efficiency of the generator is increased by efficient secondary electron suppression and low operational pressure. The generator is currently being tested at moderate power levels to ensure the voltage holding and beam power handling capabilities. With the beam power of only $500 \mathrm{~W}$ the generator produces $2 \times 10^{8} \mathrm{n} / \mathrm{s} \mathrm{D}-\mathrm{D}$ neutrons. The beam power will gradually be raised to the currently available, high voltage power supply limited level of $6 \mathrm{~kW}$. This beam power will give neutron yield of approx. $2-3 \times 10^{9}$ $\mathrm{n} / \mathrm{s}$ D-D neutrons, which corresponds to the radiation safety limit of the facility at current shielding thicknesses.

\section{Moderator/Shielding Structure}

The moderator structure functions as a radiation safety shielding as well as it has a port for thermalized neutrons. The shielding structure consists of 12" of polyethylene surrounded with $20 \mathrm{~mm}$ of lead. The polyethylene works as a moderator for the neutrons and the lead acts as a shield for the low energy $\mathrm{x}$-rays produced in the generator as well as the gamma rays produced in the moderator. The current shielding enables the generator to operate continuously at the level of $10^{\circ} \mathrm{n} / \mathrm{s}$ and it is certified and approved by the Environment Health and Safety group. The shielding opens to enable access to the neutron generator.

\section{Power, Utilities and HV Shielding}

The neutron generator is evacuated using a turbo molecular pump, which is situated outside the radiation shielding enclosure to minimize the activation of the pump components. The high voltage safety includes an interlocked safety cage that surrounds the radiation safety shield. The operation of the generator is done from a control rack, which houses the RF- and HV-power supplies as well as the vacuum instrumentation and various temperature monitors. There are also different gamma and neutron radiation monitors that are connected to a computer enabling a remote monitoring of the facility via local Ethernet. In figure 4 a 3D layout drawing of the neutron generator test stand is shown.

\section{Prompt Gamma Activation Analysis}

Construction of a PGAA and Neutron Activation Analysis (NAA) experimental station consisting of a portable 8centimeter-diameter neutron generator and polyethylene moderator was completed. Necessary RWA plans have been developed to ensure safe experimental operation, and an induced radiation checklist has been developed for experimental approval. Our participation in an International Atomic Energy Agency Coordinated Research Project (IAEA CRP) for "The Characterization of Legacy-Material Inventories and Wastes by Prompt and Delayed Gamma Activation Analysis (PGAA/NAA)" with the neutron generator has been approved. An HPGe detector is being provided by collaborators at LLNL for performing the measurements at the experimental station required for the IAEA CRP.

\section{Cell Studies}

Based on the development of a small diameter neutron probe by Dr. Leung, a collaboration was begun with the Department of Radiation Oncology at UCSF to explore the feasibility of neutron brachytherapy for the treatment of prostate cancer. An interdisciplinary team of physicians, physicists, engineers and radiobiologists from UCSF and LBNL have been meeting on a regular basis to forge this collaboration, and the grant-writing effort it will require for funding. In addition, the available neutron beam will allow the completion of feasibility experiments for grant applications to DOE and NASA. Recent calls for proposals from each of these agencies have interest in the occupational risks associated with exposures to low doses of low flux neutrons from neutrons at power-generating reactors, or from neutron exposures in space flight. Finally, there is the possibility of investigating the use of neutrons for analyzing the elemental composition and intracellular localization of elements within individual cells.

\section{Neutron Laboratory}

The new type of neutron generator offers an opportunity to set up low-cost neutron laboratories for science and teaching. A conceptual study was performed and a White Paper titled "Multi-Purpose, Low-Cost Laboratory for Neutron Science" was written and submitted to the DOE Department of Basic Energy Sciences. Combined with neutron scattering instrumentation, specifically designed to take advantage of the unique properties of the neutron 


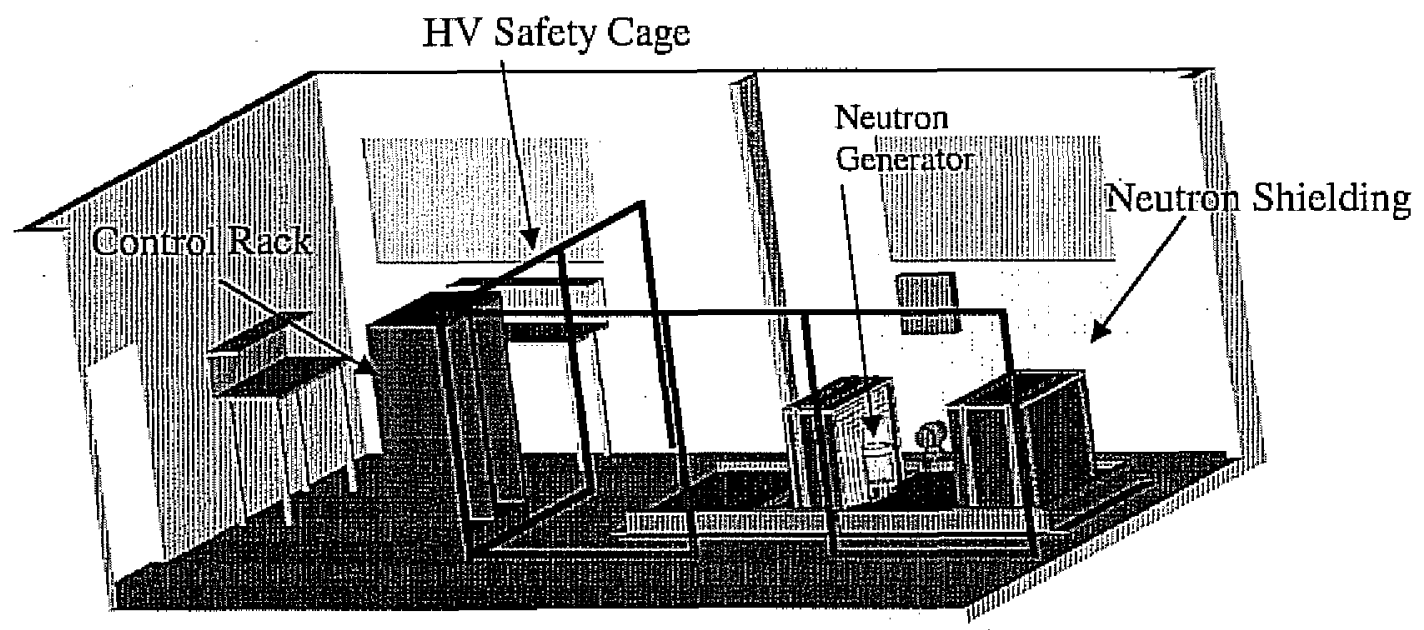

Figure 4: The layout of the neutron facility, the neutron generator and the radiation shielding on right surrounded with the HV safety cage and on the left high voltage rack and some work benches.

source, such a laboratory could be used for research in materials science, structural biology, and other areas. Applications other than neutron scattering include instrumentation development, activation analysis, radiography, radiation biology, and medicine. Such neutron laboratories would be ideal for teaching purposes and could be built at research universities across the country.

\section{Publications}

K.N. Leung, B. Ludewigt, J. Vujic, P. Peterson, "MultiPurpose, Low-Cost Laboratory for Neutron Science," White Paper to be submitted to DOE Department of Basic Energy Sciences.

\section{Advanced Simulation of Complex Beam Systems}

Principal Investigators: Phillip Colella, Eric Esarey, Alex Friedman, Robert Ryne, and Jean-Luc Vay

Project No.: 01002

\section{Project Description}

This work has two important strategic goals:

- First, to provide a computational foundation for the two key scientific areas critical to the development of the next generation of particle and laser-beam based accelerators: (I) wave-particle interaction in the full six-dimensional phase-space of multi-particle, nulti-mode particle-wave systems; and (2) coulomb and electromagnetic space-charge-dominated transport of highintensity, charged particle beams. These will have immediate applications in the studies of laserplasma acceleration, acceleration and transport of heavy ions for fusion, and simulations of intense bunches in circular accelerators including threedimensional (3D) space-charge effects.

- Second, to build a capability that combines accelerator physics with applied mathematics, numerical algorithms, and advanced computing, to address the nation's next-generation programs in accelerator science and technology.

This project brings together theoretical and experimental beam physicists, computational scientists, and computer scientists to address some of the most critical problems in beam science. Although all the accelerator/beam systems have unique features that require special techniques for simulation, there are many computational tools and techniques that are common to all these systems and to 
many others. Our approach is to identify and develop tools that can be adopted to meet the needs of a broad spectrum of particle beam simulation projects. The primary focus will be on tools that significantly enhance code performance, permit codes to be readily ported to the highest performance machines at the National Energy Research Scientific Computing (NERSC) Center and elsewhere, and allow accelerator scientists to use advanced visualization techniques to understand complex beam systems. These tools will be turned into components that can be used by a number of simulation projects. Some of the techniques to be developed are general, and will have applicability to a broad range of emerging research areas at Berkeley Lab and elsewhere.

Particle-in-cell simulations of laser and plasma-based advanced accelerators will be carried out based on the code XOOPIC. Originally, XOOPIC was developed for a variety of non-accelerator applications (e.g., plasma processing, plasma display panels, microwave tubes). Under this program, XOOPIC will be modified to allow efficient simulation of advanced accelerators. This will include implementation of a moving window, i.e., a simulation domain that moves with the beam at the speed of light; generalization of the electron beam and laser pulse launchers; inclusion of collisional models valid for highly relativistic electrons; and further optimization for efficient performance on massively parallel platforms. Simulation of relevant advanced accelerator configurations will be carried out.

We will complete the integration of the WARP particle-incell beam simulation code with the Adaptive Mesh Refinement (AMR) package Chombo, via a scripting level interface based on the Python language. This requires significant modification of both code packages, and development of the data-passing and parallel domaindecomposition management. Introducing AMR into codes for accelerator modeling has the potential for reducing by an order-of-magnitude or more the amount of computer time required to perform such calculations. Using the new capability, we will carry out simulations of space-charge dominated beams in injectors and accelerating structures with greater fidelity and efficiency than has heretofore been possible, with relevance to a number of applications for advanced accelerators.

\section{Accomplishments}

On the numerical simulation of laser-plasma accelerators, progress has been made in three areas.

- A fully nonlinear Maxwell-fluid code, the first of its kind that does not use simplifying approximations (e.g., time-averaging over the fast laser frequency, quasi-static approximations, mildly relativistic limit), was developed to model ultra-intense laser-plasma interactions. This code is being used to study the propagation of laser pulses in plasma channels and to design an optimized single-stage of a laser wakefield accelerator.

- Ionization models, both tunneling (field-induced) and collisional, have been integrated into the code Object-Oriented Particle In-Cell (OOPIC) code in collaboration with Tech-X Corp. and the U. Colorado. This code has been used to model jonization-induced blue shifting of a laser pulse propagating through a gas jet. Good agreement has been obtained between the numerical results and the laser Optics and Acceleration Systems Integrated Studies (I'OASIS) lab experimental results.

- A semi-analytical code has been developed to model the effects of space charge on the evolution of ultra-short electron bunches with large energy spreads. This code sorts the longitudinal momentum distribution into narrow bins, calculates the space charge force in the rest frame of each bin by assuming an ellipsoidal spatial charge distribution, and then sums the space charge force from each bin to update the motion of the electrons. This code is being used to study the evolution of electron bunches in vacuum after they emerge from the plasma in the l'OASIS laser wakefield accelerator experiments.

Progress was made on two fronts in the development of an AMR-based modeling capability for intense beams in accelerators:

- A complete set of routines were written to give the Chombo AMR field solver access to the WARP database containing the description of the conductor geometry. The information is used by Chombo for modeling intemal conductors via the Shortley-Weller approximation, and is provided for a given grid patch upon Chombo's demand. This scheme provides a clear separation of tasks between WARP and Chombo: WARP manages the geometry of internal conductors, while Chombo manages the hierarchy of grids used by the Poisson solver. We have also written a specification for particle-in-cell calculations for Chombo, and are implementing it.

- A prototype axisymmetric $(r, z)$ Poisson solver was built on the foundations of WARP's existing $(r, z)$ multigrid Poisson solver. While the productionlevel general three-dimensional AMR-Poisson solver is being developed as part of the Chombo 
package, this prototype is allowing us to explore the benefits of AMR on injector simulation. In this problem, $_{*}$ very fine resolution is needed around the emitting surface, as well as at the beam front and edges, while a coarser grid can be tolerated elsewhere. The benefits of mesh refinement were demonstrated on a steady-state axisymmetric calculation of a beam diode. A one-dimensional longitudinal (z) solver with full AMR capability was also implemented in WARP and the first highfidelity time-dependent simulation of a beam generation and propagation with zero rise-time was obtained.

In the area of modeling high intensity beams in circular accelerators, we have successfully combined the capabilities of two important beam dynamics codes: IMPACT, a particle-in-cell code for modeling high intensity linacs, and MaryLie, a nonlinear beam optics code based on Lie algebraic methods. To accomplish this, it was necessary to develop "sliced" versions of all thick beamline elements. In particular, a sliced version of combined function dipole magnets was developed in order to model accelerators such as the Fermi National Accelerator Laboratory (FNAL) booster and the Brookhaven National Laboratory (BNL) booster which contain this type of magnet. We also developed and implemented a procedure based on a split-operator symplectic integration algorithm to include the effects of space charge. The resulting code combines the physics of both computer models, and represents a single, comprehensive code for modeling low and high intensity beams in a wide range of linear and circular of accelerators.

\section{Publications}

B.A. Shadwick et al., "Fluid Simulations of Intense Laserplasma Interactions," IEEE Trans. Plasma Sci. 30, 38, 2002.

D.A. Dimitrov et al., "Simulations of Laser Propagation and Ionization in l'OASIS Experiments," AIP Conference Proceedings on Advanced Accelerator Concepts, 647 (Amer. Inst. Phys., 2002), pp. 192-202, 2002.

G. Fubiani et al., "Semi-analytical 6D Model of Space Charge Force for Dense Electron Bunches with a İarge Energy Spread," AIP Conference Proceedings on Advanced Accelerator Concepts, 647 (Amer. Inst. Phys., 2002), pp. 203-212, 2002.

J.L. Vay et al., "Mesh Refinement for Particle-In-Cell Plasma Simulations: Application to Heavy Ion Fusion," Laser and Particle Beams, 2002, in press.

J.L. Vay et al., "Progress in the Study of Mesh Refinement for Particle-In-Cell Plasma Simulations: Application to
Heavy Ion Fusion," 7th International Computational Accelerator Physics Conference proceedings, 2002, in press.

\section{Solid State Quantum Computer Development with Single Ion Implantation}

Principal Investigators: Thomas Schenkel and Jeffrey Bokor

Project No.: 02002

\section{Project Description}

The focus of this project is experimental studies of fundamental device physics critical for the development of a prototype solid state quantum computer (SQC). Several promising schemes for the realization of scalable SQC's are based on arrays of single donor atoms (e. g. ${ }^{31} \mathrm{P}$, or ${ }^{125} \mathrm{Te}$ ) embedded in a solid matrix (e. $\mathrm{g} ., \mathrm{Si}$, or $\mathrm{Si}_{\mathrm{x}} \mathrm{Ge}_{\mathrm{y}}$ heterostructures). To date, theoretical studies by far outnumber experimental programs in solid state quantum computing. Our approach is based on the formation of qubit arrays by single ion implantation. The development of integrated qubit arrays is aimed at gaining experimental data that enable the leup from device proposals to the design and testing of prototype solid state quantum computers.

Arrays of qubits are formed by single ion implantation of ${ }^{31} \mathrm{P}^{15+}$ ions at the Electron Beam Ion Trap facility. Using highly charged ions for implantation allows the detection of single ion impacts with $100 \%$ efficiency. This enables the formation of non-Poissonian array structures, where exactly one, rather than on average one ion is implanted into a given device area. A combination of focused beam and mask technology is used for pattern definition at the 100 nanometer level. Through detailed characterization of the electrical properties of qubit arrays, we determine defect levels and optimize processing steps in relation to requirements for functional device elements. These studies provide data for the design of a processing scheme for the integration of qubit arrays into a functional, prototype solid state quantum computer test structures. Here, pairs of single electron transistors will be formed in silicon on insulator substrates and integrated with control gates and pairs of ${ }^{31} \mathrm{P}$ atoms.

\section{Accomplishments}

In our first year, we have moved from a conceptual design stage into experimental studies of device and processing modules that are vital for the development of state quantum 
computer structures for ${ }^{31} \mathrm{P}$ qubits in group IV semiconductor (such as $\mathrm{Si}$ and $\mathrm{SiGe}$ ). We have begun with the detailed characterizution of very low dose, low energy ( 2 to $15 \mathrm{keV}$ ) phosphor implants in silicon wafers as a function of annealing conditions. The goal here is to identify annealing conditions that maximize the electrical activation of dopant atoms while keeping diffusion at a minimum. Initial results indicate a strong dose dependence of the electrical activation fraction during rapid thermal annealing (e. g., at $1000^{\circ} \mathrm{C}$ for $10 \mathrm{~s}$ ). A qubit spacing of about 20 nanometers corresponds to a dose of only $2 \times 10^{11}$ ${ }^{31} \mathrm{P} / \mathrm{cm}^{2}$, and we have identified co-implantation of silicon ions as a means to achieve high electrical activation fractions. Based on these first results we have developed a processing scheme for the integration of gate and readout structures with ion implanted single atom arrays. The first test structures will be pairs of ${ }^{3 \mathrm{P}} \mathrm{P}$ qubits with pairs of single electron transistors. We have identified a processing protocol for single electron transistor formation that is compatible with ion implantation and annealing. Single electron transistors will be used as highly sensitive electrometers for the detection of spin dependent charge transfer events (an indirect way to single spin detection). We have formed the first single electron transistor pairs in silicon on insulator wafers with Electron Beam Lithography (Nanowriter in Center for X-Ray Optics, Materials Sciences Division) (see Figure 5). We are now working on optimization of the processing modules needed to complete the SET processing. This work is makes use of the processing capabilities at Berkeley Lab and at the UC

\section{Publications}

T. Schenkel, A. Persaud, S.J. Park, J. Meijer, J.R. Kingsley, J.W. McDonald, J.P. Holder, J. Bokor and D.H, Schneider, "Single Ion Implantation for Solid State Quantum Computer Development," Journal of Vacutum Science and Teclmology $B, 20,2819$ (2002) in press.

T. Schenkel, J.P. Holder, J.W. McDonald, J. Meijer, A. Persaud, D.H. Schneider, "Single Ion Implantation for Solid State Quantum Computer Development," Quantum Dot Devices and Computing, Proceedings of SPE, V4656, 2002.

T. Schenkel, E. Stach, A. Persaud, and S.J. Park, "Formation of Nanometer Scale Holes in Thin Membranes with a Dual Beam FIB," Applied Physics Letters, in preparation.
Microlab. The field of quantum computer development is booming and this project positions us well to contribute to the many daunting problems on the path to the realization of the tantalizing possibilities of quanturn computation.

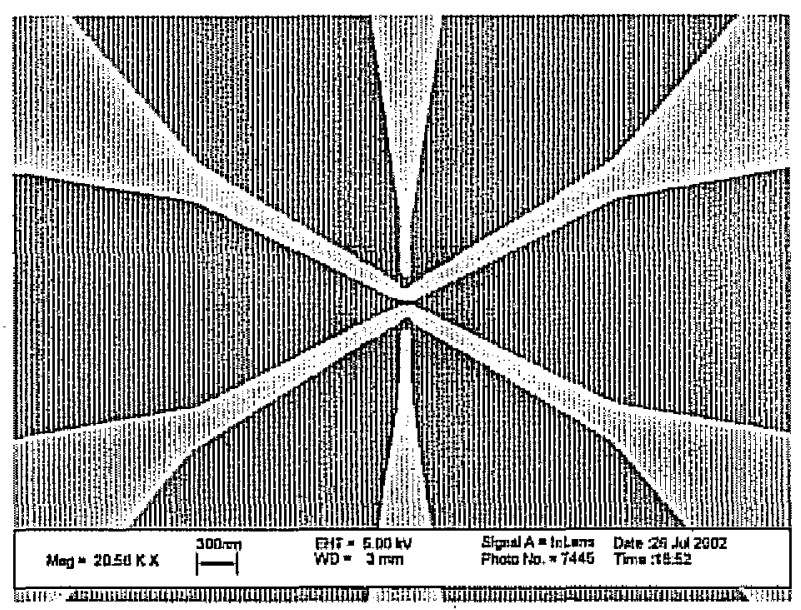

Figure 5: Layout of a pair of single electron transistors formed in silicon-on-instlator by electron beam lithography and plasma etching (A. Liddle, J. Bokor and T. Schenkel).

\section{Synchrotron and Wiggler Radiation Measurement of the Longitudinal Bunch Distribution in Hadron Colliders}

\author{
Principal Investigators: William Turner
}

Project No.: 02003

\section{Project Description}

The purpose of this proposal is to develop a concept for the measurement of the longitudinal bunch distribution of protons and heavy ions in hadton colliders. Emphasis will be placed on developing a concept that can operate over it wide range of operating conditions from injection to top energy from commissioning to full luminosity and for protons and heavy ions. Such a device would find a wide range of applications that provide essential information for machine operation including measurements of: (1) the untrapped beam fraction, (2) the intensity of ghost bunches, (3) the fraction of particles in the beam abort gap, (4) the longitudinal bunch shape and (5) coherent multi bunch 
modes. The information provided by such a device is necessary to provide information for protecting the collider from extensive damage by the large stored energy of the circulating beam [ 360 megajoules per proton beam in the case of the Large Hadron Collider (LHC) under construction at the European Laboratory for Particle Physics near Geneva, Switzerland (CERN)].

The approach that will be investigated has two parts; (1) utilization of the synchrotron and wiggler radiation emitted by highly relativistic proton and heavy ion bunches in strong magnetic fields and (2) cross correlation of the synchrotron and wiggler radiation with a laser pulse that is phase locked to the storage ring radio frequency (rf) system. A prototype system will be assembled and tested at the Advanced Light Source (ALS) electron storage ring at Berkeley Lab.

\section{Accomplishments}

The method of longitudinal density measurement uses the non-linear mixing the synchrotron radiation from the bunched beam of the ALS with a mode locked solid-state laser oscillator and detection of the up-converted radiation with a photo-multiplier tube. The laser operates at $71.428 \mathrm{MHz}$ (14 natnoseconds) with pulse width 50 femtoseconds, wavelength $800 \mathrm{~nm}$ and power $100-200 \mathrm{~mW}$. The circumference of the ALS has 328 if buckets separated by 2 nanoseconds so that after 7 revolutions each if bucket has been sampled once. The laser is phase locked to the ALS if frequency. The laser frequency is phase modulated with a mirtor mounted on a piezo-electric crystal and arranged to sample the profile of each if bucket with 32 time slices separated by approximately 4.8 picoseconds. Nominally 276 consecutive buckets are filled with $\sim 1.2 \mathrm{~mA}$ per bunch and the 52 bucket gap has a single "cam shaft" bunch - 6mA near the center of the gap for purposes of synchronization. Our main objective in FYO2 was to develop the electronic hardware and the software necessury to measure the longitudinal density profile of the ALS that has just been described.

The primary accomplishment was the development of the electronic hardware and software for a proof of principle experimental measurement of longitudinal density. A data acquisition board was designed and fabricated to accumulate the number of photon counts per time slice per bunch passage. The data acquisition board contains an address generator and bunch accumulation memory that keeps the data sorted according to position in the circumference of the ALS. Data accumulation stops and a trigger is generated for readout by a personal computer (PC) after a certain number of revolutions of the ALS. The software that has so far been developed allows for display of electron density profile with 4.8 picoseconds resolution over the full circumference of the ALS, peak and integrated intensity per if bucket versus bucket position and ms pulse width per rf bucket versus bucket position.

The data acquisition board and software described in the previous paragraph have been successfully tested in initial experiments on the ALS. Fig. 6 shows the density profile of the "cam shaft" pulse in photon counts per bunch passage for a typical fill of the ALS. The rms pulse width is approximately 18 picoseconds. Similar density profiles of the complete if bucket train of the ALS have been obtained. A number of interesting effects observed including deviations in bunch intensity, systematic increase of $\mathrm{rms}$ bunch length toward the bunch gap, leakage of electrons from the "cam shaft" bunch into the trailing if bucket and decrease of bunch length when the third harmonic cavity is dropped out. The power of the method is clearly indicated in Figure 6. The time resolution exceeds that possible by purely electromagnetic pickup devices and comparable time resolution has previousiy been possible only with streak cameras. The advantage of the present technique is the ease of routine digital signal analysis of a very large number of buckets $(3,564$ in the case of the future LHC).

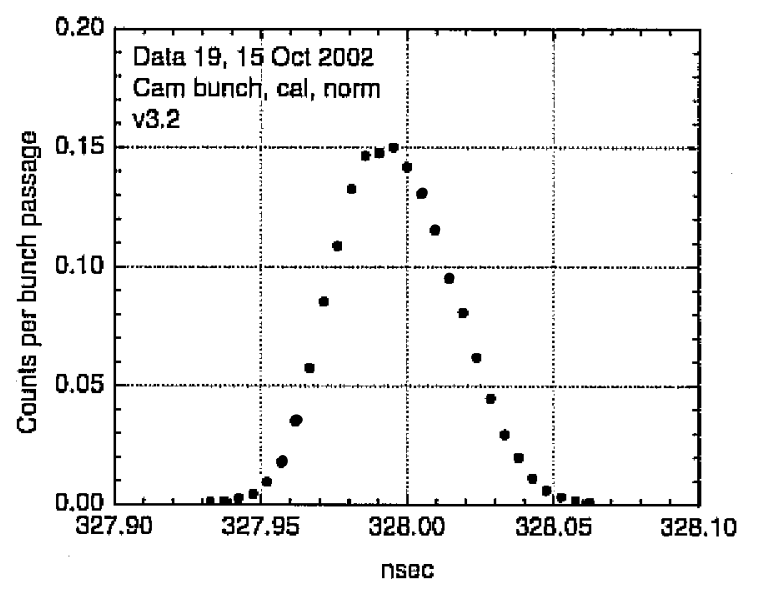

Figure 6: Beam intensity profile of a single bunch in the $A L S$ measured by correlation of synchrotron light emission with a mode locked solid-state laser oscillator.

\section{Publications}

M. Zolotorev, J.F. Beche, P. Datte, S. De Santis, P. Denes, M. Placidi, A. Ratti, V. Riot, R. Schoenlein, and W.C. Tumer, "Measurement of Longitudinal Beam Density via the Non-linear Mixing of Synchtrotron Radiation with a Mode Locked Laser Oscillator," 2003 Particle Accelerator Conference, May 12-16, 2003, in preparation. http://www-conf.slac.stanford.edu/pac03/ 


\section{A Source of Far Infrared Radiation Based on a Material with an Optically Switched Permittivity}

Principal Investigators: Max Zolotorev, Robert

Schoenlein and Alexander Zholents

Project No.: 01005

\section{Project Description}

The purpose of this work is the design and demonstration of a laser-assisted source for electromagnetic radiation in the far-infrared region based on a material with a high threshold for breakdown under electric field and optically switched permittivity. The goal is to reach $\sim 1$ gigavolt per meter $(\mathrm{GV} / \mathrm{m})$ of the radiation field near the surface of the material. This radiation field can be used for high-energy gain acceleration in a miniature, pencil-size accelerating structure-the electromagnetic wave undulator-and as an electron source with an ultra-low electron beam emittance. The long-term goal foresees the accelerator as a highenergy physics and/or a compact tabletop source of femtosecond $\mathrm{x}$-ray pulses, utilizing self-amplified, spontaneous emission of electrons in interaction with the above-mentioned electromagnetic wave undulator.

This research will include three components: (1) development and characterization of a material capable of radiating a field of $-1 \mathrm{GV} / \mathrm{m}$, (2) study of the radiation mechanism based on the abrupt change in material permittivity, and (3) a proof-of-principle experiment. Theoretical and computational tools will be developed with an aim to design, model, and analyze a new source of farinfrared radiation based on an optically switched permittivity of the material. The proof-of-principle experiment will be conducted using a colored glass filter as a model of the desired material. Ways to design a new material will be explored.

\section{Accomplishments}

The second year of this project had additional highlights:

- New THz radiator design was implemented. Small gap (5 micron) radiators were manufactured with the help of UC Berkeley Microfabrication Laboratory and Xiaofan Meng. Gold electrodes were deposited on a Gallium Arsenide (GaAs) wafer using photolithography.

- Experimental THz generation and detection setup to test new GaAs small gap radiators is nearly complete. Once complete this setup will enable testing $\mathrm{THz}$ properties of new materials (i.e. absorption, index of refraction).

- High voltage electrodes were designed and manufactured with the help of Design Works group and Dan DeBoer from Design and Fabrication group of Engineering Division.

- The high voltage of up to $10 \mathrm{kV}$ was applied without the electric break up on a gap of 125 microns when the gap between electrodes was filled with epoxy.

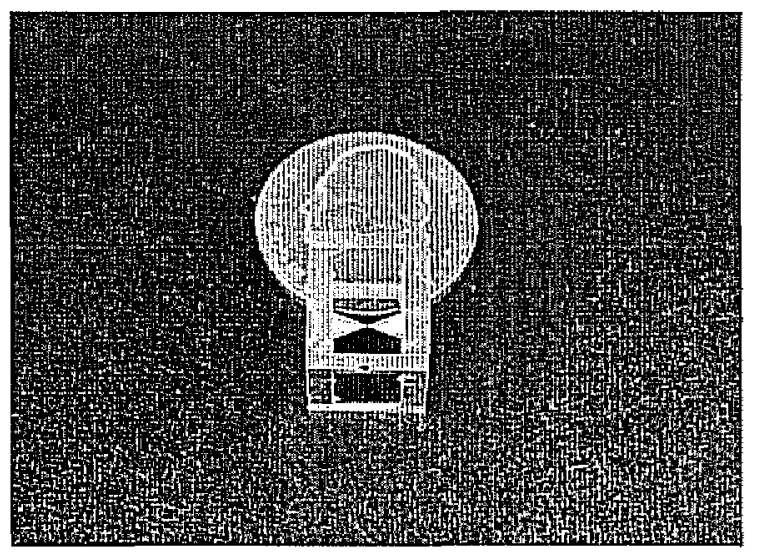

Figure 7: The electrodes shown in the figure were designed and manufactured with the help of DesignWorks group and Dan DeBoer from Design and Fabrication group of Engineering Division. The high voltage up to $10 \mathrm{kV}$ was applied without the electric break-up on a gap of 125 microns when electrodes were filled with the epoxy. 


\section{Advanced Light Source Division}

\section{Filling in a "Big Gap": A Novel Momentum-Resolved Inelastic Soft $X$ - Ray Emission Spectrometer for the Study of Strongly Correlated Quantum Systems}

Principal Investigators: M. Zahid Hasan, Jonathan Denlinger, James Underwood, Zhi-Xun Shen, and Zahid Hussain

Project No.: 01006

\section{Project Description}

Despite extensive research efforts, the electronic structure of strongly correlated quantum systems continues to be a major class of unsolved problems in physics. The essential physics of these systems are believed to be dominated by the strong Coulomb interactions among relatively localized valence electron (d-electrons in the case of, for example, transition metal oxides). Well-developed momentumresolved spectroscopies such as photoemission and neutron scattering cannot directly probe valence charge-charge correlation (fluctuation) spectrum in a momentum-resolved manner, as angle-resolved photoemission probes the singleparticle occupied states and neutrons do not couple to the electron's charge directly. Optical Raman and infrared spectroscopies measure charge-fluctuation spectrum (occupied to unoccupied) but they are confined to the zone center ( $q-0$ ) hence not momentum-tuned. A good understanding of momentum-resolved charge-charge correlation function is of paramount importance to gain insights into the charge-transport mechanisms in correlated systems. In addition, there is no momentum-resolved bulk spectroscopy to study the unoccupied states. To fill in this gap in electronic spectroscopies we are building a novel soft x-ray spectrometer that is 100 to 1000 times more efficient than previous designs.

Previous hard $\mathrm{x}$-ray $(-10 \mathrm{keV})$ resonant inelastic experiments at the transition metal $K$ edges have demonstrated its applications for the study of momentum structure of the upper Hubbard band in cuprates. Exciting results have also been obtained regarding excitons in correlated systems and their dispersion. However, the resonant scattering at the transition metal $L(2,3)$ edges promise to be very spectacular and should also, according to the theory, have at Ieast several orders of magnitude more scattering intensity in the inelastic channel. It would be invaluable to be able to look at the charge, lattice, or spin excitation in highly correlated materials with resolution limited only by the finite temperature of the sample.

\section{Accomplishments}

As briefly described in last year's report, the prototype soft $x$-ray emission spectrometer has been manufactured. The test results for individual component are:

- The spherical mirror has been measured to have the correct radius 11.026 meters. The roughness and slope error of the mirror are minimized to achieve better focusing. These mirrors are currently coated with $\mathrm{Ni}$.

- The variable line spacing (VLS) plane grating, with ruling density 1200 lines/mm for higher energy application, was obtaitued. The atomic force microscope (AFM) image reveals high quality ruling pattern and the efficiency, measured at beam line 6.3.2, is between 5-10\%. The peak efficiency is around $800 \mathrm{eV}$, which is optimized for studying late $3 \mathrm{~d}$ transition metal oxides. The master grating is currently coated with Au. The lower energy grating, with 600 lines/mm ruling density, has been designed and ordered.

- The in-vacuum, back-illuminated CCD camera has been tested to achieve the designated sensor temperature, $-75^{\circ} \mathrm{C}$. The minimum dark current (thermal noise) Ievel is lower than 0.002 electrons/pixel/s, which is critical for enhancing signal/noise ratio for such photon-hunger experiments.

The spectrometer chamber, coupling vacuum chamber section, pumping section, test stand and test chamber have been manufactured and assembled at beam line 6.3.2 for alignment test. The source used in the test is the specular reflection from a $38 \mathrm{fm}$ wire. The alignment was done with zeroth order light of the spectrometer. The results are summarized as follow:

- According to the ray-tracing program (SHADOW) and analytical calculations, the produced image width should be approximately 110 um (the demagnification ratio is -3 ). By varying the mirror angles, the minimum width obtained in the test is about $160 \mu \mathrm{m}$ (FWHM), which is only $50 \%$ larger than the "perfect" value. 
- The grating angle is relatively insensitive and the image is well focused on the detector.

- The focal depth, at the zeroth order, can be up to $+/-3 \mathrm{~cm}$. This is consistent with the SHADOW simulation.

The test chamber, under the upgrade process, will have an extra $10 \mu \mathrm{m}$ slit installed between the spectrometer and the photon beam. With the slit acting as the source, the first order light of the spectrometer can be tested. Such test will tell us the resolving power of the spectrometer, as well as the calibration between grating angles and photon energies, which can then be used in data analysis.

\section{Development of a Far-IR Synchrotron Radiation Source at the ALS}

Principal Investigators: Michael Martin, John Byrd, Wayne McKinney, David Robin, Alexander Zholents, and Robert Schoenlein

Project No.: 02004

\section{Project Description}

The purpose of this project is twofold: (1) to further our understanding of the generation of coherent synchrotron radiation through experiments at the Advanced Light Source (ALSS) using unique modes of operntion of the ring, and (2) to consider a pre-conceptual design of a dedicated infrared storage ring to be located at the ALS. A source in the far infrared (IR) that is much brighter than what is available at present will open up new possibilities for research in this traditionally difficult energy range. We intend to characterize super-radiant far- $\mathbb{R}$ radiation from various novel modes of ring operations, including further experiments with femtosecond laser slicing. This research will be carried out with the goal of understanding how to create stable, coherent far-IR radiation from a storage ring. This will also lead to generation of a pre-conceptual design for a possible future infrared storage ring.

\section{Accomplishments}

Super-radiant, temporally coherent synchrotron radiation (CSR) could significantly enhance the available photon flux at far-infrared wavelengths, a traditionally difficult frequency range. At wavelengths longer than the electron bunch length in a storage ring the individual electrons radiate in phase. This means that the radiative power in this long-wavelength regime is proportional to the square of the number of electrons, as opposed to the linear relationship that is well known with conventional (incoherent) synchrotron light sources. Even at very low beam currents, the number of electrons is quite large so the resultant flux enhancement is huge. In FY02, work was carried out in order to more fully understand the CSR effect in storage rings. We have achieved several significant results.

In order to demonstrate the very high powers possible from $\mathrm{CSR}$ in the $\mathrm{THz}$ frequency range, we collaborated with researchers at Jefferson Lab (Virginia) and Brookhaven National Lab (New York) to make measurements of the CSR power and spectrum from a new energy recovery linac (ERL) located at Jefferson Lab. This ERL is capable of accelerating -500 fs electron bunches with a relatively high $75 \mathrm{MHz}$ repetition rate therefore relatively high average current due to the use of superconducting RF accelerating cavities. The spectral output of the CSR coming from this source compared to a conventional thermal $\mathbb{R}$ source is shown in Figure 1, panel (a). At the low wavenumber (long wavelength) end of the spectrum, one can see that the gain by using a CSR source is about six orders of magnitude in intensity, in good agreement with predictions. The high power of this source was, however, hampered by poor noise qualities probably due to the fact that each new electron bunch is slightly different from the previous one. These results were recently published in Nature.

Since noise is a primary concern for IR spectroscopy, we believe a storage-ring based CSR source would be inherently a cleaner source. However one needs to understand just how short electron bunches can be compressed for stored bearn, and how much current one can then obtain. At quite high single-bunch currents the electron bunches become unstable and begin to emit bursts of CSR. In this project, we have made detailed characterizations of the beam parameters for when this bursting regime occurs, and we compare these measurements to a model predicting the onset of self-amplified microbunching in stored electron bunches in Figure 1, panel (b). The excellent agreement with no adjustable parameters shows that we really understand the mechanism behind this bursting instability threshold and can now determine optimized parameters for achieving stable high-power CSR in a storage ring source. These results were recently published in Physical Review Letters. 

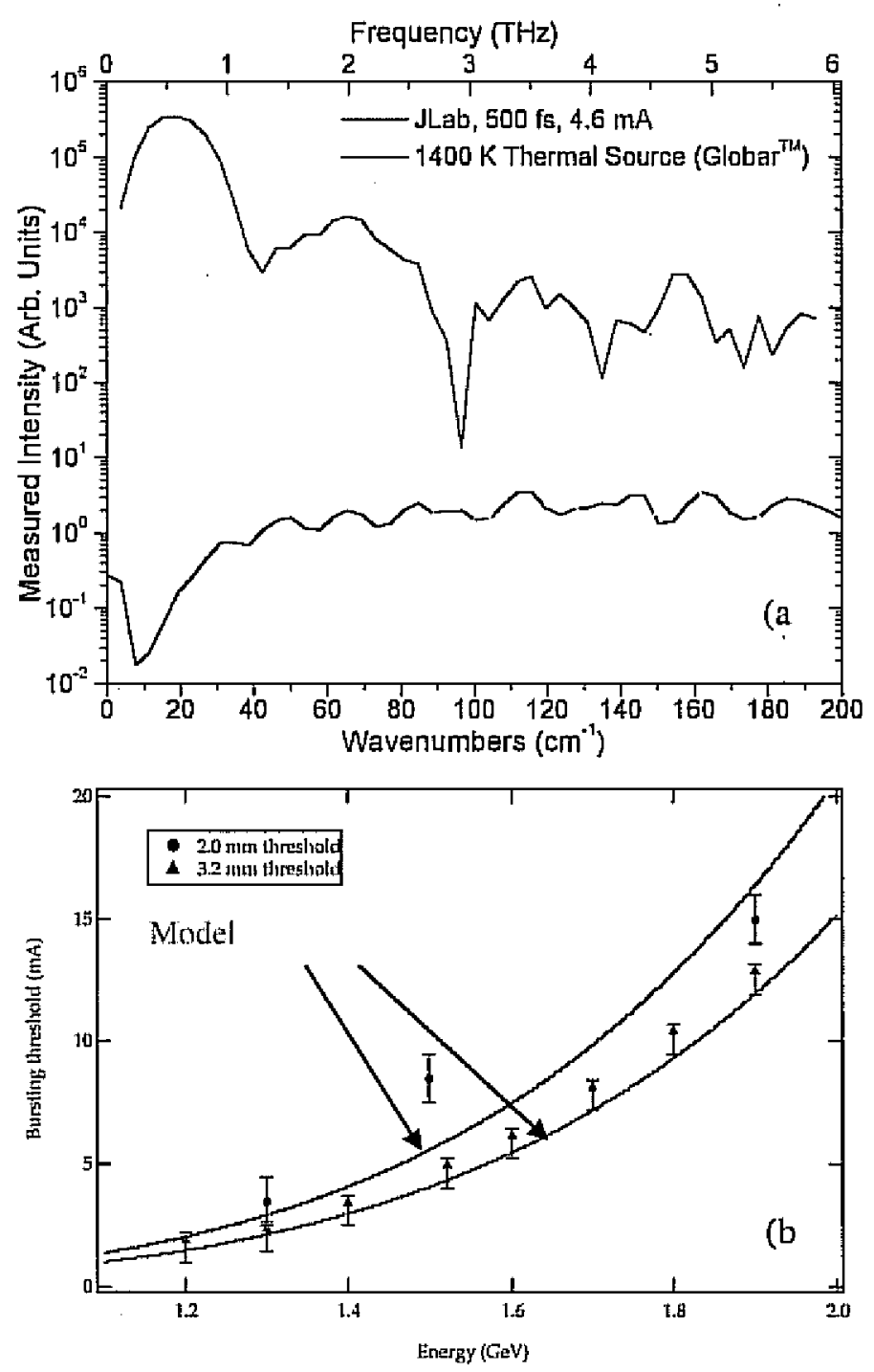

Figure 1: (a) Measured CSR intensity versus frequency from, JLab ERL source compared to a conventional themal IR source. Note the log intensity scale. (b) CSR bursting threshold as a function of electron beam energy at 3.2 and $2 \mathrm{~mm}$ wavelengths. Data are shown as points with error bars. Theoretically calculated threshold using nominal ALS parameters at 3.2 and 2 mm wavelengths are shown as solid lines with no adjustable parameters.

\section{Publications}

G.I. Carr, Michael C. Martin, Wayne R. McKinney, K. Jordan, George R. Neil and G.P. Williams, "High-Power Terahertz Radiation from Relativistic Electrons," Nature 420, 153-156, (2002), November 14, 2002.

http://dx.doi.org/10.1038/nature01175

G.L. Carr, Michael C. Martin, Wayne R. McKinney, K. Jordan, George R. Neil and G.P. Williams, "Very High Power THz Radiation at Jefferson Lab," Physics in Medicine and Biology 47, 3761-3764 (2002), November 7, 2002. http://stacks.iop.org/0031-9155/47/3761
J.M. Byrd, W. Leemans, A. Loftsdottir, B. Marcelis, M.C. Martin, W.R. McKinney, F. Sannibale, "Observation of Broadband Self-amplified Spontaneous Coherent Terahertz Synchrotron Radiation in a Storage Ring," Phys. Rev. Lett. 89, 224801 (2002), November 2002. http://ink.aps.org/abstract/PRL/v89/e224801

W.C. Barry, et al., "A Dedicated Storage Ring for Far-IR Coherent Synchrotron Radiation at the ALS," 2002 EPAC (European Particle Accelerator Conference) proceedings, June 2002.

http://infrared.als.lbl.gov/pubs/EPAC_2002_IR_Ring.pdf 


\section{High-Pressure Research at the ALS}

Principal Investigators: Howard Padmore, Paul Alivisatos, Raymond Jeanloz, and Simon Clark

Project No.: 01008

\section{Project Description}

The goal of this work is to perform technical demonstrations using the Advanced Light Source (ALS) for high-pressure physics, geophysics and chemistry. The research will be centered on understanding structural and chemical dynamics in nanocrystalline materials, and examining the chemical reactions that occur at high pressure and temperature inside the Earth and planets. This work will also strengthen the support of the highpressure research community for a dedicated superbend beamline for this type of research at the ALS.

Our approach is to use an existing beamline at the ALS and equip it with the necessary hardware to perform high pressure and temperature experiments, using the probes of x-ray absorption spectroscopy to deduce chemical state, large-angle scattering to determine atomic packing, and small-angle scattering to determine the shape of molecular assemblies. This is being made possible by the move of another research group onto a dedicated beamline, therefore freeing space and beamtime. We also intend to make use of the adjoining laser lab for a high-power laser for sample heating, and we already have much of the infrastructure, such as a ruby fluorescence system for pressure measurement and diamond anvil cells for application of pressure. A particular thrust of this program will be to develop $x$-ray spectroscopy at energies $<13$ $\mathrm{keV}$, requiring thin diamonds or the use of beryllium gaskets in transverse mode.

\section{Accomplishments}

We have established a prototype high-pressure capability on beamline 7.3.3. This included developing highly automated data collection procedures, expanding the user community, and preparing papers for submission. Studies include:

- Earth Science: Studies of solid oxygen, mantle rocks and hydrous minerals.
- Chemistry: Studies of phase transitions and bulk modulii of nano-particles.

- Physics: Studies of strengths of materials.

We started a high pressure (HP) infrared program by completing demonstration experiments, and by designing dedicated HP end station with Lawrence Livermore National Laboratory. Furthermore, we started a HP single crystal diffraction program with demonstration experiments to begin early 2003.

We have also established a high-pressure support laboratory. This provides the necessary cell loading equipment, and the building up of a laser heating system.

Considerably more details of the scientific accomplishments will be in the journal articles now being prepared. For follow-on activities, we attracted funding for a dedicated superbend beamline (12.2.2) scheduled for first user beam in fall 2003. This project was pivotal in obtaining National Science Foundation (NSF) funding to support Earth Science HP science at the ALS, paying for a laser heating system, a beamline scientist and associate beamline scientist (appointments start early 2003). We also attracted additional funding from Livermore $(\$ 0.5 \mathrm{M})$ to complete superbend end station and one beamline scientist over five year period to support HP research at the ALS, as well as additional support for an expert in laser beating of diamond anvil cells for 1 year.

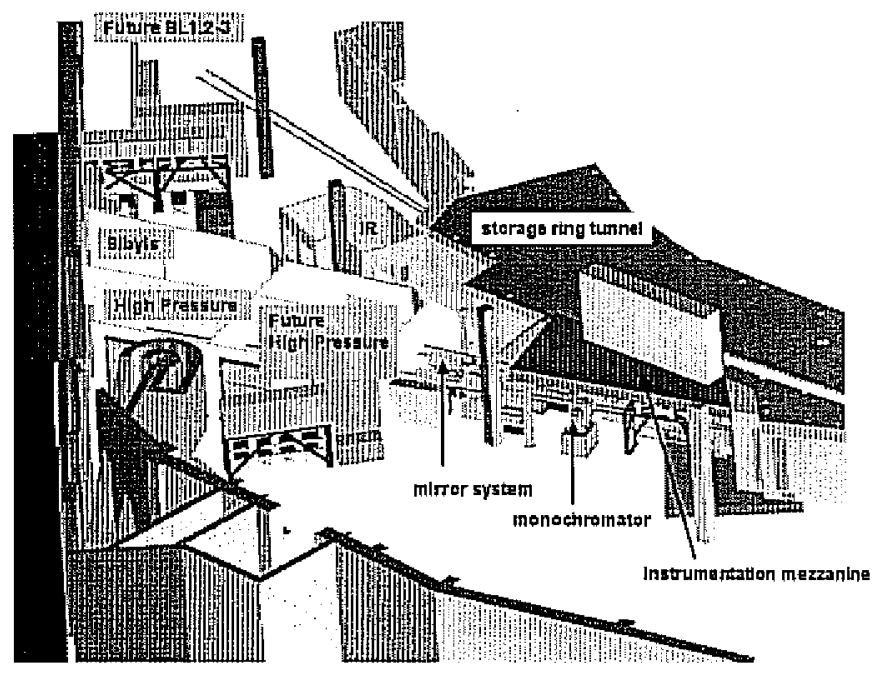

Figure 2: The dedicated high-pressure beamline 12.2.: under construction. 


\section{Publications}

Bernedetti et al., "Solid Oxygen at STP: a Dense Phase of Oxygen Formed at High Pressures and Temperatures,"

Science, 2003, in preparation.

Zaziski et al., "Kinetics of CdSe Nanorod Transformations Under Pressure," Physical Review Letter, 2003, in preparation.

S.M. Clark et al., "A New High Through-put High-pressure Diffraction Facility at the Advanced Light Source," $J$. of Synch. Rad., 2003, in preparation.

Prillman et al., "Size Dependence of the Phase Transition in Nanoparticle Fe203," Physical Review Letter, 2003, in preparation.

Prillman et al., "Nature of the Phase Transition in Nanoparticle Fe2O3," Physical Review Letter, 2003, in preparation.

Prillman et al., "Bulk Modulus of TiN Nanoparticles," Physical Review B., 2003, in preparation.

Bertone et al., "Enhanced Bulk Modulus in Quartz Nanoparticles," Physical Review B., 2003.

\section{Spatially Resolved X-ray Absorption and Diffraction Applied to Problems in Environmental Science}

Principal Investigators: Howard Padmore, Garrison Sposito, and Alain Manceau (CNRS, U. Grenoble)

Project No.: 02005

\section{Project Description}

The purpose of this project is to demonstrate the application of micro-focused $x$-ray absorption spectroscopy (XAS) and $\mathrm{x}$-ray diffraction (XRD) to problems of significance in environmental and geosciences. The project will concentrate on problems related to toxic cleanup, and in particular to the mechanisms of sorption of transition metals by natural systems. Successful demonstration of this type of work should address a new community of users. Control of the mobility of contaminants in a natural environment requires a detailed knowledge of the molecular mechanisms of trace element reactions with mineral phases.

Progress in this area requires use of a probe that can give local chemical-state information of a contaminant and substrate, as well as local structure information on the mineral phase present. This has to be achieved on a relevant length scale, in this case the scale of mineral grains, or inhomogeneous components in soil, typically a few microns. This can be done using a combination of microfocusing and XAS-XRD. The aim here is to demonstrate that worthwhile information can be extracted that will be relevant to remediation. We will work with Professor A. Manceau, who has pioneered use of XAS and XRD for these problems, and will extend this work to the micron spatial domain in this project.

\section{Accomplishments}

The long-range goal of the work carried out by Alain Manceau's team at the Advanced Light Source (ALS) is to understand and control the form of metal contaminants in the environment. Human activities have introduced large concentrations of metals in soils and sediments, and the design of appropriate clean-up actions and the improvement of our current technology rest on the detailed characterization of the fate of metals in these formations. Indeed, the toxicity of metals largely depends on their forms, the rule being that the less soluble form is also the less toxic. Therefore, the potential human and ecological impacts of hazardous heavy metals can be addressed by transforming soluble species to sparingly soluble forms, either in-situ or in landfills after excavation. Our main effort at the ALS has been to develop the synergetic use of three of the most powerful non-invasive techniques, namely synchrotron $x$-ray fluorescence analysis (SXRF), scanning $x$-ray diffraction (SXRD), and extended $x$-ray absorption fine structure (EXAFS), all applied at the micrometer-scale of resolution so that we can measure and correlate the speciation of contaminants in a natural environment, ie. measure the local chemistry at work in a complex medium of mixed minerals. The following 4 subtopics have been pursued;

- Natural sequestration of trace contaminants ( $\mathrm{Ni}$, $\mathrm{Zn}$, As...) in soils,

- Transfer and speciation of zinc in a dredged sediment deposit and in the subsoil,

- Attenuation of the environmental mobility of $\mathrm{Zn}$ by phytostabilization and mineral amendments,

- Speciation of $\mathrm{Zn}, \mathrm{Mn}$ and $\mathrm{Fe}$ in a soil-root system.

Under this project, we have made excellent progress in addressing some of the key questions above, and gone a long way in establishing ALS as a center for microstructural and chemical measurements applied to problems in environmental science. This has helped to establish a vigorous user community, and will be critical in establishing the scientific and technical case for moving the activity to a superbend beamline. One example is given 
here from the first subtopic, illustrative of the general type of work that was performed in this project.

\section{Molecular-scale Speciation of $Z n$ and $N i$ in Soil Ferromanganese Nodules from Loess Solls of the Mississippi Basin}

Determining how environmentally-important trace metals are sequestered in soils at the molecular scale is critical to developing a solid scientific basis for maintaining soil quality and formulating effective remediation strategies. The speciation of $\mathrm{Zn}$ and $\mathrm{Ni}$ in ferromanganese nodules from soils of the Mississippi basin was determined by a synergistic use of three non-invasive synchrotron-based techniques: $\mathrm{X}$-ray microfluorescence; $\mathrm{X}$-ray microdiffraction; and extended $\mathrm{X}$-ray absorption fine structure spectroscopy. We show that $\mathrm{Ni}$ is distributed between goethite $(\alpha-\mathrm{FeOOH})$ and the Mn oxide, lithiophorite, whereas $\mathrm{Zn}$ is bound to goethite, lithiophorite, phyllosilicates, and the Mn oxide, birnessite. The selective association of $\mathrm{Ni}$ with only $\mathrm{Fe}$ and $\mathrm{Mn}$ oxides is an explanation for its higher partitioning in nodules over the soil clay matrix reported from soils worldwide. This could also explain the observed enrichment of $\mathrm{Ni}$ in oceanic manganese nodules. Using micro-SXRF, the distribution of $\mathrm{Fe}, \mathrm{Mn}$, and $\mathrm{Ni}$ in a soil ferromanganese nodule were determined. Two-dimensional XRD patterns were characterized by the presence of point diffraction spots from sub-micron crystals, including quartz, feldspars, titanium oxides, illite and kaolinite, and continuous Debye rings arising from nanometer-sized particles, including phyllosilicate, goethite ( $\alpha-\mathrm{FeOOH})$, and three Mn oxides, lithiophorite, birnessite, and Fe-vernadite. The mineral abundance maps of the first four finely divided (nanometersized) species were produced by integrating at each pointof-inalysis diffracted intensities of ( $h k l)$ reflections along Debye rings. The comparison of the $\mu$ SXRF and $\mu S X R D$ maps clearly shows that nickel and lithiophorite have the same contour maps, therefore suggesting that $\mathrm{Ni}$ is bound to this particular nanophase. However, it should be noted that an association between an element $E$ and a mineral $M$ does not necessarily imply that $E$ is chemically bound to or included in the structure of $M$. Still, $\mu$ EXAFS spectroscopy showed that $\mathrm{Ni}$ is indeed included in the $\mathrm{MnO}_{2}-\mathrm{Al}(\mathrm{OH})_{3}$ mixed-layer oxide lithiophorite structure and substitutes for $\mathrm{Mr}^{3+}$ in the manganese layer. Our systematic screening of ferromanganese nodules already resulted in the identification of two predominant $\mathrm{Zn}$ and $\mathrm{Ni}$ species: $\mathrm{Zn}-$ birnessite, and (Ni,Zn)-lithiophorite. Ni has a high affinity for lithiophorite, and this association has become a standing observation independently of the origin of samples. We intend in the future to elucidate the reason for this high selectivity. In contrast to nickel, $\mathrm{Zn}$ can be bound to several mineral species (essentially $M n$ oxides), and this multiplicity can be explained by the electronic structure of $\mathrm{Zn}^{2+}$ which, owing to the completeness of its d orbitals $\left(\mathrm{d}^{10}\right.$ configuration), has no preferred crystal field stabilization energy in either tetrahedral or octahedral coordination. $\mathrm{Zn}^{2+}$ may fill in a variety of crystallographic sites with birnessite and lithiophorite. More on this work can be found at http://www-lgit.obs.ujf-grenoble.fr/users/manceau.
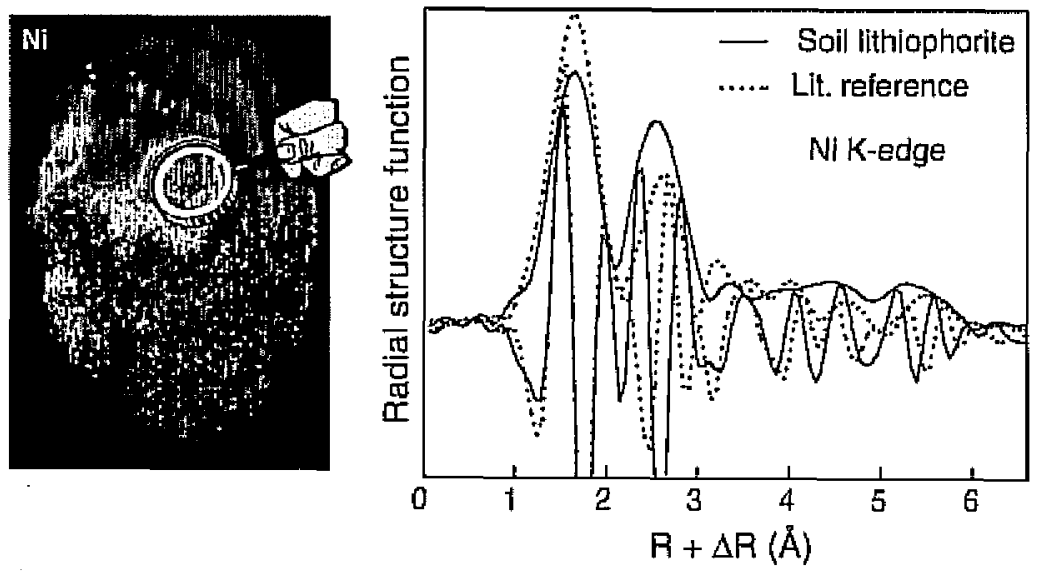

Lithiophorite reference
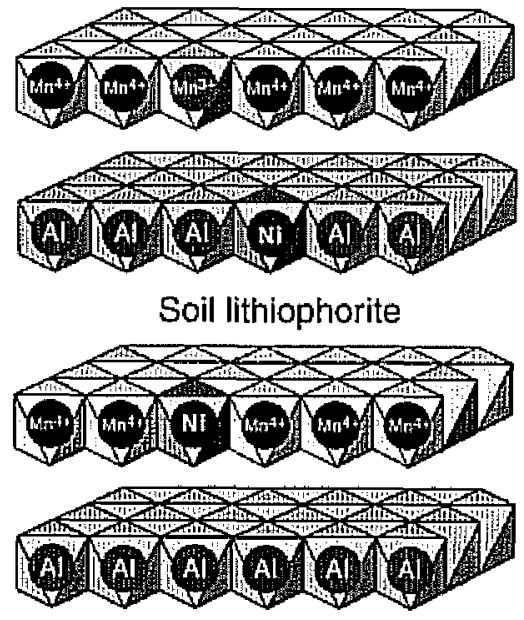

Figure 3: Molecular-level incorporation mechanism of nickel in soil lithiophorite by Ni K-edge $\mu E X A F S$ spectroscopy. Shown is the Fourier transform (modulus plus imaginary part) from a 'hot spot' of the core of a soil ferronanganese nodule, compared to the Ni-edge data from a Ni-containing lithiophorite reference, in which Ni substitutes for $\mathrm{Li}$ in the Al(OH) layer. 


\section{Publications}

S. Hlawatsch, M, Kersten, C.D. Garbe-Schb̈nberg, F. Lechtenberg, A. Manceau, N. Tamura, D.A. Kulik, J. Harff, and E. Suess, "Trace Metal Fluxes to Ferromanganese Nodules from the Western Baltic Sea as a Record for LongTerm Environmental Changes," Chemical Geology, 182, 697-710, 2002.

M.P. Isaure, A. Laboudigue, A. Manceau, G. Sarret, C. Tiffreau, P. Trocellier, G. Lamble, J.L. Hazemann, and D. Chateigner, "Quantitative Zn Speciation in a Contaminated Dredged Sediment by $\mu \mathrm{PLXE}, \mu \mathrm{SXRF}$, EXAFS

Spectroscopy and Principal Component Analysis," Geochimica et Cosmochimica Acta, 66, 1549-1567, 2002.

M.P. Isaure, A. Laboudigue, A. Manceau, G. Sarret, C. Tiffreau, and $\mathrm{P}$. Trocellier, "Characterisation of Zinc in Slags Issued from a Contaminated Sediment by Coupling $\mu \mathrm{PIXE}_{+} \mu \mathrm{RBS}, \mu \mathrm{EXAFS}$ and Powder EXAFS Spectroscopy," Nuclear Instruments and Methods in Physics Research 181, 598-602, 2001.

A. Manceau, N. Tamura, R.S. Celestre, A.A. MacDowell, N. Geoffroy, G. Sposito, and H.A. Padmore, "Molecularscale Speciation of $\mathrm{Zn}$ and Ni in Soil Ferromanganese Nodules from Loess Soils of the Mississippi Basin," Environimental Science \& Techology, 2002 in press.

A. Manceau, N. Tamura, M.A. Marcus, A.A. MacDowell, R.S. Celestre, R.E. Sublett, G. Sposito, and H.A. Padmore, "Deciphering Ni Sequestration in Soil Ferromanganese Nodules by Combining X-ray Fluorescence, Absorption and Diffraction at Micrometer Scales of Resolution," American Mineralogist, 87, 1494-1499, 2002.

G. Sarret, P. Saumitou-Laprade, V. Bert, O. Proux, J.L. Hazemann, A. Traverse, M.A. Marcus, and A. Manceau, "Forms of Zn Accumulated in the Hyperaccumulator Arabidopsis Halleri," Plant Plysiology, 2002, in press.

N. Tamura, R. Spolenak, B.C. Valek, A. Manceau, N. Meier Chang, R.S. Celestre, A.A. MacDowell, H.A. Padmore, and J.R. Patel, "Submicron X-ray Diffraction and its Applications to Problems in Materials and Environmental Science," Review in Scientific Instrumentation, 73, 1369-1372, 2002.

A. Manceau, M.A. Marcus, and N. Tamura, "Quantitative Speciation of Heavy Metals in Soils and Sediments by Synchrotron X-ray Techniques. In Applications of Synchrotron Radiation in Low-Temperature Geochemistry and Environmental Science, P. Fenter, and N.C. Sturchio, Eds. Reviews in Mineralogy and Geochemistry, Mineralogical Society of America, Washington, DC, vol. 49, 341-428, 2002.
More on this work can be found at http://www-lgit.obs.ujfgrenoble.fr/users/manceau.

\section{Simulations of Femtosecond X-ray Spectra of Photoexcited Molecules}

Principal Investigators: Shaul Mukamel (U. Rochester), Robert Schoenlein, and Thornton Glover

Project No.: 02006

\section{Project Description}

The goal of this project is to develop a theoretical framework for simulating and interpreting ultrafast $x$-ray absorption spectra on the 100 -femtosecond time scale. This theoretical work is driven by new experimental capability for generating ultrashort $x$-ray pulses from the Advanced Light Source (ALS). Time-resolved spectroscopy with femtosecond $x$-ray pulses will open new areas of research in physics, chemistry, and biology by allowing the motion of atoms in materials to be disectly probed on the time scale of a vibrational period, which is the fundamental time scale for structural dynamics. At present, there is a strong need for theoretical support to interpret time-resolved $x$-ray absorption spectra.

Nonlinear spectroscopy in the visible regime is successfully described using the well developed machinery of nonlinear response functions (NRF), which are expressed as combinations of multiple-time correlation functions of the dipole operator and provide the most compact and general formulation of optical signals. This research will focus on extending this approach to coherent nonlinear ultrafast $x$ ray spectroscopies. The various signals in terms of the NRF will be represented as combinations of multiple-time correlation functions of the current and the charge-density. $\mathrm{X}$-ray techniques thus probe the dynamics of charge and current densities rather than of the dipole. Since charge distributions are directly related to chemical bonding and structure, the creation and breaking of bonds and other electronic and nuclear motions can be monitored with femtosecond time resolution.

\section{Accomplishments}

This project helped establish a collaboration between the theory group of Prof. Shaul Mukamel at the University of Rochester, and the ultrafast $\mathrm{x}$-ray spectroscopy group at Berkeley Lab. A highly-qualified postdoc (from the theory 
group of J. Rehr at the University of Rochester) has been recruited. His background is in x-ray absorption theory and modeling, and during this first part of the LDRD he has been at U. Rochester learning the machinery of nonlinear response functions and the corresponding computer codes that have been developed in the Mukamel group.

In particular, density functional theory (DFT) potentials have been applied with the coherent electronic oscillator (CEO) method of finding excited state potentials. The aim of this work is to use this CEO routine to make a molecular dynamics model of optically excited states for carrying out simulations of the $x$-ray spectra of these optically excited molecules. The ground state DFT potentials are being taken from existing quantum chemistry codes (e.g. Gaussian).

In addition, the suitability of existing $\mathrm{x}$-ray spectroscopy codes is being tested. Of particular interest is the near edge region of molecules on which experiments of optically excited states have already been performed, and whose geometry is known in both the ground and excited states. So far, the spectra of Nickel Tetraphenylporphyrin (both with and without axial piperidine ligands) have been computed.

\section{Picosecond (P-sec) Time-Resolved Photo-Electron Emission Microscopy on Magnetic Nano-Structures}

Principal Investigators: Andreas Scholl, Aaron Lindenberg, Howard Padmore, and Joachim Stöhr

Project No.: 02007

\section{Project Description}

The purpose of this work is to pioneer the study of ultra-fast magnetic processes with a combination of temporal and spatial resolution hitherto inaccessible to the magnetic materials research community. Understanding the dynamics of the magnetization process is one of the fundamental issues in magnetism research and it is of great importance for technological applications. Of key interest is the dynamics of magnetization-precession and magnetizationdamping which govern the speed of magnetization reversal in magneto-electronic devices.

This work will also provide a foundation for nextgeneration microscopes such as PEEM3. We will use an existing photoelectron microscope, PEEM2, for imaging, and use a combination of the native bunch structure of the Advanced Light Source (ALS) (50-picosecond x-ray pulses) and a picosecond rise-time magnetic pulse system for pump-probe magnetization studies. The fast magnetic pulse for the "pump" will be generated by the interaction of a picosecond laser with a semiconductor photo-switch, and this will provide current to a lithographically defined microcoil to provide the field. The system will also be deposited on the semiconductor surface, at the center of the microcoil. The laser system will be locked to the revolution frequency of the ALS, and a programmable delay between magnetic field pump and $x$-ray probe will allow us to map out the spatially resolved dynamics of the system.

\section{Accomplishments}

During the year we have successfully tested and evaluated a novel experimental technique for the investigation of processes on a p-sec time scale and a sub-micron. We have carried out first proof-of-principle experiments. A postdoc with experience in magnetization dynamics research was hired who developed the technique, designed samples and conducted benchmark experiments. During the first phase of the project, an existing Ti-sapphire laser system was synchronized with the synchrotron radiation source (Advanced Light Source). External control of the delay between the laser pulse, which serves as a trigger or pump. and the $\mathrm{x}$-ray pulse, which serves as a probe, was demonstrated on a fast $x$-ray diode. Samples incorporating photoconductive switches, wave-guide structures, and magnetic layers and patterns were fabricated in collaboration with Stanford Linear Accelerator Center (SLAC). The ALS photoemission electron microscope PEEM-2 was modified for time-tesolved experiments and a control software was developed. A detail of the wave-guide structure is shown below.

The operation and potential of the technique was evaluated in three stages: (1). Measure synchronization and jitter of pump and probe using a fast oscilloscope, (2). Determine time-zero and magnetic pulse shape using PEEM imaging, and (3). Image magnetization processes in micron-sized magnetic patterns. The synchronization of the pump and probe pulse was evaluated by correlating the optical and the $x$-ray pulse on a fast $x$-ray diode. Furthermore the occurrence of the laser pulse was compared to a reference signal, provided by the ALS. In both cases we determined very low jitter, better than the desired time resolution of 100 picoseconds. The shape and temporal position of the magnetic field pulse, generated by the photoconductive switch and the wave-guide was determined in-situ using the PEEM microscope. The electric and magnetic field generated by the current pulse leads to an image deflection. The result of the data analysis is plotted below (open dots), demonstrating a time resolution of currently about 150 picoseconds. 
The magnetization dynamics of CoFe was studied, demonstrating the capability of the technique to determine fast magnetization processes. The presence of the field pulse led to a fast reorientation of the CoFe layer magnetization parallel to the applied field when the pump and the probe pulse overlapped. The evolution of the local magnetization in a sub-micron area is compared to the field pulse in the graph below. In summary, we have successfully demonstrated the potential of a novel time-resolved x-ray imaging technique, which allows the investigation of nanoscale objects with p-sec time resolution.

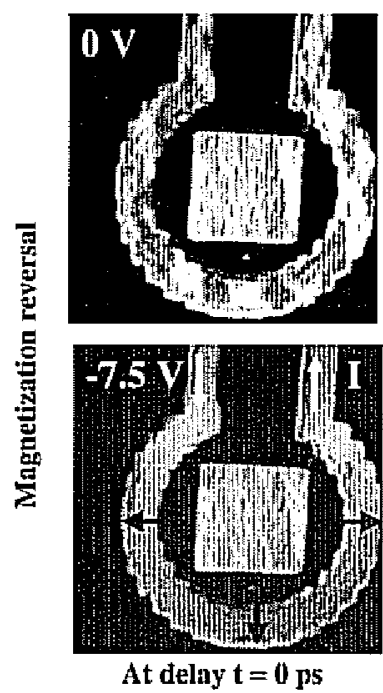

First x-ray microscopic measurements demonstrating p-sec magnetization relnxation

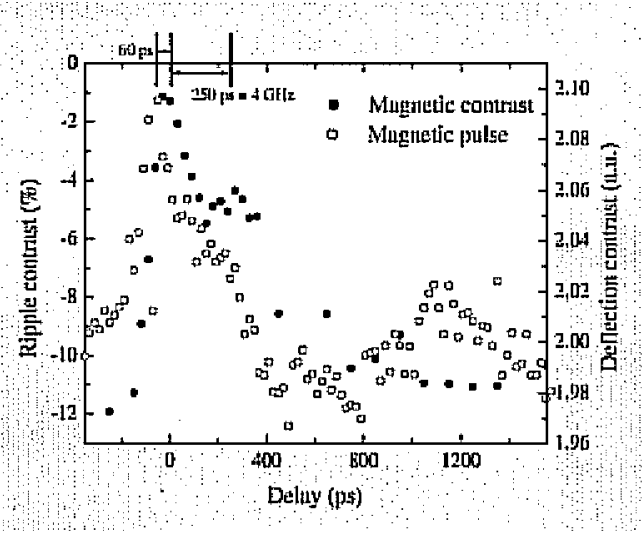

Figure 4: Inaging p-sec magnetization processes. The images on the left show the domain structure of a loop (20 $\mu$ diam.) without (top) and with (bottom) a magnetic field pulse. The graph on the right shows the time dependence of the field pulse (open dots) and the magnetization (dots).

\section{Publications}

A. Scholl, S.B. Choe, Y. Acremann, A. Lindenberg, C. Stamm, S. Andrews, H.C. Siegmann, J. Stöhr, and H. A. Padmore, "A New Time Resolved Experiment for the Microscopic Study of Magnetization Dynamics Using XPEEM," Workshop on New Opporturities in Ultrafast Science using X-rays, April 14-17, 2002, conference presentation.

\section{Bonding in Low-Dimensional Structures: Theory and Computation}

Principal Investigators: Michel Van Hove, Andrew Canning, and Lin-Wang Wang

Project No.: 02008

\section{Project Description}

This theoretical and computational project will interpret and analyze data to understand the bonding between atoms in low-dimensional structures. The results, based on experiment, will be fundamental to the design and exploitation of novel nanoscale structures and processes. In its first year, the work will focus on three state-of-the-art experiments: (1) quantum-well states relevant to magnetism at metallic interfaces; (2) structural studies of nanoparticlemolecule interactions that control nanoparticle growth mechanisms. The long-term benefit will be theoretical and 
computational capabilities that greatly enhance the value of a broad range of namoscale studies aimed at designing new devices.

The project joins theoretical and computational expertise in the Materials Sciences Division and the National Energy Research Scientific Computing Center with experimental programs in several Berkeley Iab divisions. Large-scale, first-principles calculations will be applied to complex structures to model and understand their bonding and properties. Advanced multiple-scattering calculations will be used to extract bonding and related electronic and magnetic information from experiments conducted at the Advanced Light Source (ALS), primarily with photoemission and $x$-ray absorption. The two sets of calculations will feed into each other at the fundamental level of quantum wavefunctions, forming a closely coherent program.

\section{Accomplishments}

During the first year of this project, we have achieved very significant results in our first study. It addressed recent experimental observations (made at the ALS by Qiu, Rotenberg et al) on quantum wells important for understanding and improving state-of-the-art magnetic storage capabilities. The goal here is to make it possible to theoretically "engineer" electronic states in a wide variety of nanoscale structures of practical relevance.

The specific system studied is an ultrathin layer of copper grown on another ultrathin layer of cobalt, which is in turn deposited on a copper substrate. The two layers exhibit the type of electronic states that govern the so-called giant magnetoresistance effect used in magnetic recording media. We have calculated the electronic states as a function of film thickness: they include states localized at the interfaces or within each layer, as well as states that extend across both layers. We are also including the local interface structures: the difference between these and the "bulk" structures of thicker samples can have important consequences on the physical properties of these films.

As a result, we can reproduce quite well the energies of the experimentally detected quantum-well states. This allows us to improve on the existing heuristic "Phase Accumulation Model" to explain the observations. In turn, this permits generalizing and offering the capability to "engineer" electronic states with desired properties.

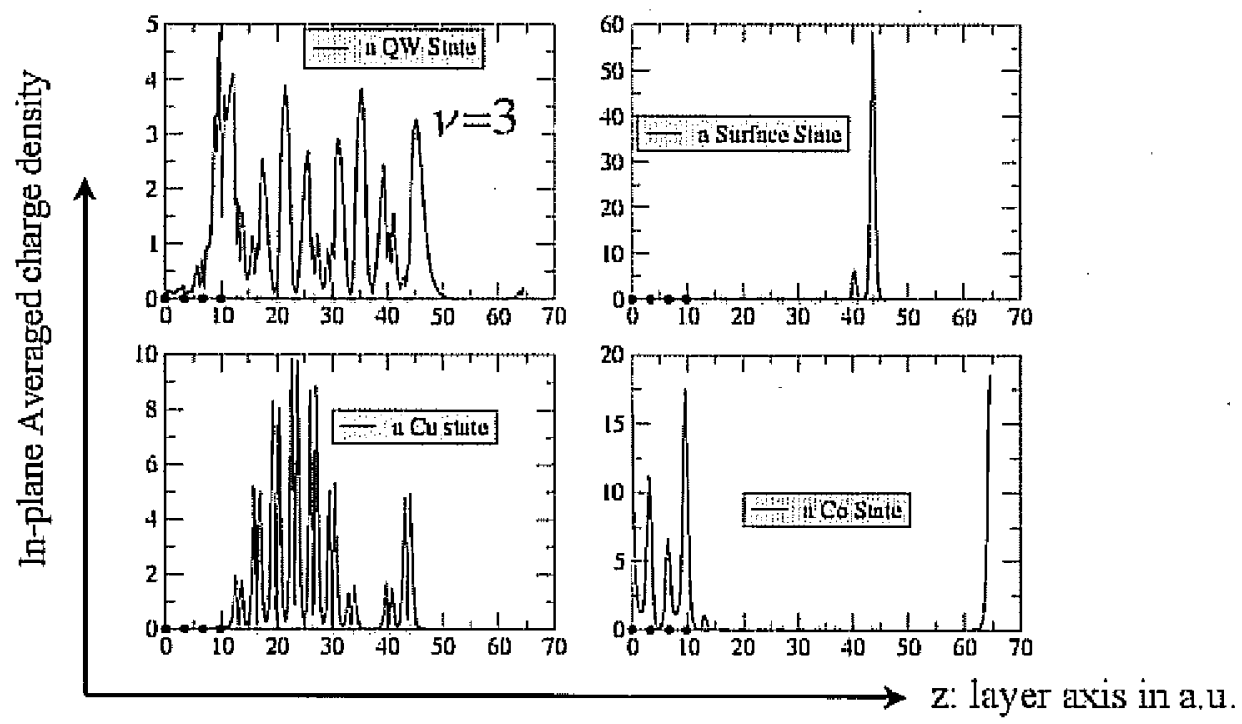

Figure 5: Theoretical quantum well states of different character for a Co4Culo film (4 atomic planes of Co and 10 atomic planes of $\mathrm{Cu}$, repeated after a slice of vacaum). Plotted is the plane-averaged electronic valence density across the quantum wells, showing where the bonding electrons are most likely to be found. Quanthm well states at left are contrasted with surface and Co substrate states at right.

\section{Publications}

J.M. An, D. Raczkowski, M. Leung, L.W. Wang, A. Canning, M.A. Van Hove, Z.Q. Qiu, Y.Z. Wu, and E. Rotenberg, "The Quantization Condition of Quantum-Well States in $\mathrm{Cu} / \mathrm{Co}(100)$, , in preparation. 


\section{Chemical Sciences Division}

\section{Disorder and Multiple Length Scales in Non-Fermi Liquid f-electron Intermetallics}

\author{
Principal Investigators: Corwin Booth
}

Project No:: 02009

\section{Project Description}

Local lattice disorder has been shown to affect the properties of Kondo systems, as well as non-Fermi liquids. Long-range disorder likewise effects magnetic systems, and therefore also affects non-Fermi liquids that are near a magnetic/non magnetic quantum critical point. Under this proposal, we will measure lattice disorder at multiple length scales from nearest-neighbor ( $\sim 0.2$ nanometers), to the $1-2$ nanometer range, as well as considering long-range order. These data should help determine whether clusters exist with long-range magnetic interactions, and thus allow for direct statements about the applicability of certain types of disorder-based models of non-Fermi liquid behavior.

There are two primary goals for this proposal. The first and most important for the first year is to develop skills in $\mathrm{x}$-ray scattering, and in particular in pair-distribution function (PDF) analysis of powder diffraction data, for measuring length scales in the 0.2-2 nanometer regime. This technique will then be used as a compliment to the shorter range information available from the $x$-ray absorption fine structure (XAFS) technique, and the average crystal structure data obtained from Rietveld refinement. The second primary goal is to further our understanding of how lattice disorder, and in particular how clustering of impurity and other disorder centers, affects the electronic and magnetic properties in non-Fermi liquid $f$-electron intermetallics. In FYO2, we focused on developing our expertise in PDF by analyzing neutron diffraction data on the spin glass $\mathrm{Y}_{2} \mathrm{Mo}_{2} \mathrm{O}_{7}$, collecting and analyzing $\mathrm{x}$-ray PDF data on a variety of $f$-electron intermetallics at the Advanced Photon Source, performing and analyzing neutron diffraction experiments for Rietveld analysis on $\mathrm{Y}_{2} \mathrm{Mo}_{2} \mathrm{O}_{7}$ and others at the Chalk River (Canada) reactor, and performing various XAFS experiments at the Stanford Synchrotron Radiation Laboratory. As well as providing information at all of the various inferesting lengths scales, these experiments are in preparation for performing the more difficult anomalous PDF experiments in FY03.

\section{Accomplishments}

A large part of our focus for FYO2 has been on the pyrochlore systems, in particular $\mathrm{Y}_{2} \mathrm{Mo}_{2} \mathrm{O}_{\%}$. We are using $\mathrm{Y}_{2} \mathrm{Mo}_{2} \mathrm{O}_{7}$ mostly as a model to explore the various experimental techniques, in part because we could obtain data quickly on this system. Powder neutron diffraction measurements were performed on the geometrically frustrated pyrochlores $\mathrm{Y}_{2} \mathrm{Mo}_{2} \mathrm{O}_{7}$ and $\mathrm{Tb}_{2} \mathrm{Ti}_{2} \mathrm{O}_{7}$. A spinglass-like behavior has been observed from $\mathrm{Y}_{2} \mathrm{Mo}_{2} \mathrm{O}_{7}$. Previous studies have shown that there is large disorder in Mo-Mo bonding and short-range antiferromagnetic spinordering occurring as high as $210^{\circ} \mathrm{K}$. Our neutron diffraction study of $\mathrm{Y}_{2} \mathrm{Mo}_{2} \mathrm{O}_{7}$ agrees with the previous studies up to $300^{\circ} \mathrm{K}$. However, we have observed a new feature around $375^{\circ} \mathrm{K}$, as shown in Fig. (c). We believe that this feature is relevant to the spin interaction. Although the measurement is statistically significant, we want to confirm this new transition with a time-of-flight neutron source, and other techniques. $\mathrm{Tb}_{2} \mathrm{Ti}_{2} \mathrm{O}_{7}$ is known as a spin-liquid system, in which spin fluctuations slow down continuously despite the short-range antiferromagnetic correlations even at the lowest measured temperatures. Such behavior should only be possible in a system that is very well ordered structurally as well as chemically. With temperature dependent powder neutron diffraction measurements from $\mathrm{Th}_{2} \mathrm{Ti}_{2} \mathrm{O}_{7}$, we found that it has a well-ordered crystalline structure and zero static disorder at all atom sites within uncertainty. We have therefore confirmed that $\mathrm{Tb}_{2} \mathrm{Ti}_{2} \mathrm{O}_{7}$ is a good system to study spin-liquid behavior. In the temperature range of $10-250^{\circ} \mathrm{K}$, we did not observe any structural transition (previous studies have reported that there is short-range antiferromagnetic order as high as 50 $\left.{ }^{\circ} \mathrm{K}\right)$. Our neutron diffraction measurements imply that the spin coupling is not strong enough to create any structural disorder.

In order to better understand the lattice disorder in the nonFermi liquid $f$-electron intermetallic UC. $u_{+} P d$ system, we have carried out high-resolution $x$-ray diffraction measurements with the fixed incident $x$-ray energy $98 \mathrm{keV}$ on 11-ID-C at Basic Energy Sciences Synchrotron Radiation Center Collaborative Access Team (BESSRCCAT), Advanced Photon Source. The data were taken at several different temperatures from $10^{\circ} \mathrm{K}-300^{\circ} \mathrm{K}$. After a background removed from raw data, the data were normalized and Fourier transformed to obtain the pairdistribution function (PDF). Data were fit with two different models: the nominal crystal structure (C15b) with $U$ on $4 \mathrm{a}$ 
sites, $\mathrm{Pd}$ on $4 c$, and $\mathrm{Cu}$ on $16 e$ sites, and a model that include some fraction of the Pd atoms, $s$, on the nominally $\mathrm{Cu} 16 e$ sites. The site interchange model has been shown from XAFS studies to best describe the structure. However, we could not draw any conclusion based solely on these fits to the PDF data regarding which model was more appropriate, at least when we only considered a single temperature. This inability is due to a strong correlation between the $\mathrm{Pd} 16$ e (the nominal $\mathrm{Cu}$ site) occupancy fraction and the displacement parameters $\left(u^{2}\right)$ of the Pd and Cu sites. Since these fits could not distinguish between the nominal and the site-interchange models at one temperature, temperature dependent displacement factors were fit with a Debye model, as shown in Fig. (a). The fits of the Debye model to the U and Pd $u^{2 \gamma}$ s implies that the site-interchange model is slightly better than the normal model. However, we have found that the Cu site displays a large amount of disorder from similar fits. We checked the Cu site in $\mathrm{YbAgCu}_{4}$ (an isostructural Fermi liquid), and found similar amount of disorder. This result suggests that the observed disorder in the Cu site is probably not relevant to non-Fermi liquid behavior, or may indicate a systematic error. We are still exploring this issue. For instance, because the measurements were performed with high energy $x$-rays, the PDF analysis might be less sensitive to $\mathrm{Cu}$ than the Pd and $\mathrm{U}$ atoms at this energy. In order to obtain better measurements of the local environment around a Pd-on-16e site, anomalous PDF analysis will be useful. By subtracting PDF data from below and above Pd $K$-edge, only those peaks in the PDF that involve Pd will remain. Such data should clarify the $\mathrm{Pd} / \mathrm{Cu}$ site-interchange, and possibly allow the determination of important details about the environment around the $\mathrm{Pd}$ atoms on the $16 e$ sites. We also studied a CeColn ${ }_{5}$ system with PDF analysis and found $\mathrm{CeCoIn}, \mathrm{h}_{j}$ having well-ordered crystalline structure that agrees well with previous studies.

X-ray absorption fine siructure meusurements at $\mathrm{Ce} L_{3^{-}}, \mathrm{Ru}$ $K$ - and $\mathrm{Rh} K$-edges and analyses were performed on CeRhRuSi 2 . In the data analysis, it was assumed that Ru or Rh are randomly distributed at the Ru/Rh site. These data are unable to discern between Ru and Rh (neighbors in the periodic table) and we did not observe any disorder around either the Ru or the Rh site. The Debye-Waller factors relative to Ce are shown in Fig. (b). The solid curve is a best fit to the Debye model and shows that the intrinsic disorder of all atom sites, $\mathrm{Ce}, \mathrm{Ru} / \mathrm{Rh}$ and $\mathrm{Si}$, relative to $\mathrm{Ce}$ are negligible. The results of the XAFS analyses of the Ru and $\mathrm{Rh} \mathrm{K}$-edges also show that the disorder of this system at $20 \mathrm{~K}$ is quite small, apart from the mixture of Rh and $\mathrm{Ru}$ on the $4 d$ site. Annealing the system from one to five weeks produced, at most, a very small ordering of the crystal, in contrast to the effect on $\mathrm{UCu}_{4} \mathrm{Pd}$. PDF data have been obtained on this system, but have yet to be analyzed.

In summary, we have made progress in all of the major areas of this proposal, namely applying new experimental techniques to the relevant materials. Further analyses of these data, as well as complementary measurements to better elucidate the intermediate length scales, will be the target of FY03.
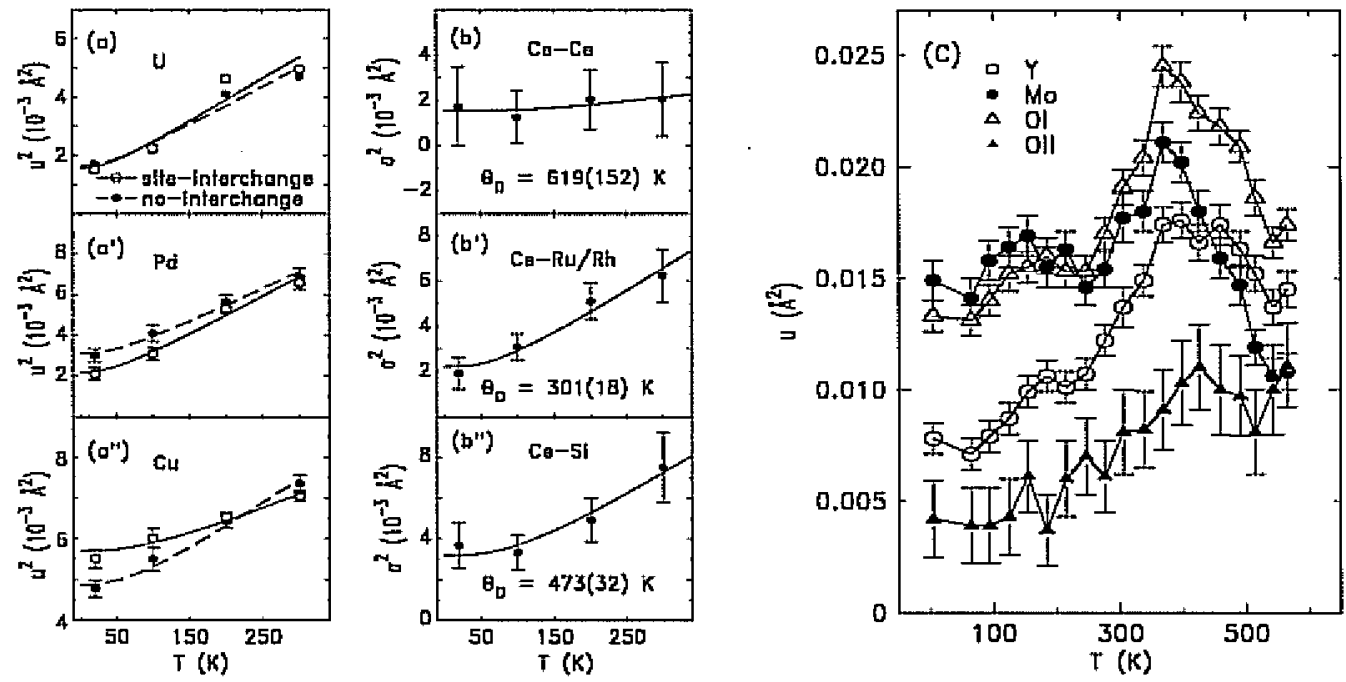

Figure 1: Displacement factors, $(a)$ for $U_{x}\left(a^{\prime}\right)$ for $P d$ and $\left(a^{\prime \prime}\right)$ for Cu from $U C u_{4} P d$, and the open circle is for the siteinterchange and closed circle is for the normal. The curves are best fits with a Debye model. (b), $\left(b^{\prime \prime}\right)$ and $\left(b^{\prime \prime}\right)$ are the DebyeWaller factors of $C e, R u / R h$ and Si respectively determined by EXAFS at Ce L3-edge. The curves are best fits and $\theta_{D}$ is Debye temperature. (c) shows the displacements factors of $\mathrm{Y}_{2} \mathrm{Mo}_{2} \mathrm{O}_{7}$ determined by powder neutron diffraction meastirenents. 


\section{Publications}

S.W. Han, C.H. Booth, E.D. Bauer, and M.B. Maple, "Temperature-dependent X-ray Diffraction Study of $\mathrm{Pd} / \mathrm{Cu}$ Site Interchange in non-Fermi Liquid $\mathrm{UCu}_{4} \mathrm{Pd}_{\text {, " SCES } 2002}$ conference proceeding, accepted for publication.

S.W. Han, J.S. Gardner and C.H. Booth, "Powder Neutron Diffraction Study of Spin-liquid Pyrochlore $\mathrm{Tb}_{2} \mathrm{Ti}_{2} \mathrm{O}_{7}$," Phys. Rev. B, in preparation.

S.W. Han, C.F. Booth, J.L. Sarrao, and J.D Thompson, "Local Order and Disorder in non-Fermi Liquid CeRhRuSi ${ }_{2+}{ }^{*}$ to be submitted to Plys. Rev. $B$, in preparation.

S.W. Han, C.H. Booth, E.D. Bauer, and M.B. Maple, "Xray Scattering Studies of the Local Pd-on-Cu Environment in non-Fermi Liquid $\mathrm{UCu}_{4} \mathrm{Pd}$, to be submitted to Phys. Rev. B, iл preparation.

\section{Development of a Neutral Molecule Synchrotron Storage Ring}

Principal Investigators: Harvey Gould and Hiroshi Nishimura

Project No.: 01010

\section{Project Description}

Our goal is to determine the feasibility and practicality of constructing molecular synchrotrons to store, slow, and cool neutral polar molecules in a way that makes it easy to use them in novel new experiments, including the formation of molecular Bose-Einstein condensates.

Laser slowing, cooling, and trapping, have not been effective for molecules because the closely spaced (vibrational and rotational) levels prevent efficient cycling transitions. This makes it possible to apply accelerator and specifically synchrotron physics and technology to polar molecules. Molecular synchrotrons have the potential to be the first widely applicable and effective method to store, slow, and cool molecules in vacuum.

Static and dynamic traps have been considered for trapping molecules. The molecular synchrotron is effectively a dynamic ( $\mathrm{rf}$ ) two-dimensional trap. It has fewer constraints than a three-dimensional trap or a static trap. A molecular synchrotron's beam energy is independent of temperature and can be varied over several orders of magnitude. It preserves a beam geometry for experiments, can have fieldfree regions, and can be scaled to accept molecules with large kinetic energies. It is well suited for evaporative cooling of the molecules. It maty be possible to use a ring as an accunnulator to increase the stored intensity.

\section{Accomplishments}

Using calculations and mathematical modeling, we have demonstrated the feasibility of constructing a synchrotron storage ring for neutral polar molecules in weak-field seeking states. The lattice is a racetrack type $3.6 \mathrm{~m}$ in circumference consisting of two of 180-degree arcs, six bunchers, and two long straight sections. Each straight section contains two triplet focusing lenses and space for beam injection and experiments.

The modeling uses deuterated ammonia molecules in a weak-field seeking state. Beam that survives 400 turns (15 s), has horizontal and vertical acceptances of $35 \mathrm{~mm}$-mr and $70 \mathrm{~mm}-\mathrm{mr}$ respectively, and an energy acceptance of $\pm 2 \%$.

Up to 60 bunches can be loaded and simultaneously stored in the ring. The molecules are injected at $90 \mathrm{~m} / \mathrm{s}$ but the velocity of the circulating beam can be decelerated to 60 $\mathrm{m} / \mathrm{s}$ after injection

The design also includes a matched injector and a linear decelerator.

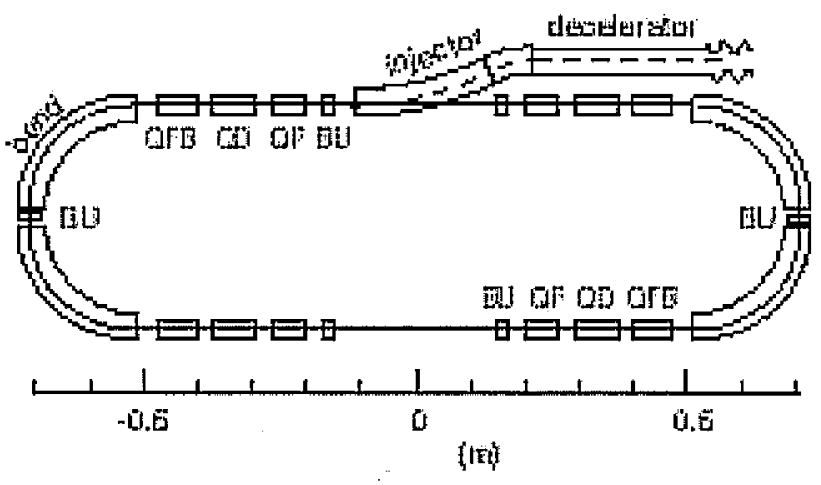

Figure 2: Schematic diagram of the synclmotron storage ring modeled in our study. The bend radius is $0.2 \mathrm{~m}$ and the circumference is $3.36 \mathrm{~m} . Q F$ and $Q B F$ are horizontal focusing (vertical defocusing) lenses, $Q D$ are horizontal defocusing (vertical focusing) lenses and $B U$ are bunchers. The injector and a portion of the decelerator are also shown. 


\section{Publications}

J. Kalnins, G. Lambertson, and H. Gould, "'Improved Alternating Gradient Transport and Focusing of Neutral Molecules," Rev. Sci. histr 73, 2557 (July, 2002).

H. Nishimuta, G. Lambertson, J.G. Kalnins, and H. Gould, "Feasibility of a Synchrotron Storage Ring for Neutral Polar Molecules," LBNL -51597, Nov, 2002, Submitted for publication to Rev. Sci. Instr.

\section{Coherent Control and Quantum Information in Polyatomic Molecules}

Principal Investigators: Stephen Leone

Project No.: 02043

\section{Project Description}

The advent of the ultrafast femtosecond laser as a tool for controlling processes such as branching ratios in chemical reactions and manipulating the evolution of vibrational wavepackets with tailored femtosecond laser pulses has provided significant insight into the complex interplay between the electronic, vibrational and rotational degrees of freedom both in diatomic and simple polyatomic systems. The present study involves stimulated Raman pumping of multimode wavepackets in the ground state of polyatomic molecules. The impulsive nature of these excitations eliminates the need for accessing any resonances and as a result will prove a general and powerful technique for looking at vibrational coherences in the ground state. Previous collaborative work has addressed the issue of multimode vibrational wavepackets in simple polyatomic systems. This project provides an extension to this work with more refined set of experiments both in state selection and probe control.

\section{Accomplishments}

In a recent collaborative effort, multimode vibrational wavepacket dynamics were observed in $\mathrm{CCl}_{4}$ using stimulated Raman excitation with the broad bandwidth output of a Ti-Sapphire laser. The present study intends to build and extend these ideas by state selection and probe control. A custom modified Spectra-Physics Regenerative Amplifier system capable of producing sub 40 femtosecond pulses with a bandwidth of 32 nanometers has recently been installed in the principal investigator's laboratory. The large bandwidth will ensure significant excitation of multiple ground state vibrational modes which can be manipulated using shaped femtosecond laser pulses. A much simpler and cost effective approach to pulse manipulation will be used based on a thin film deformable membrane mirtor. In addition, a pulsed molecular beam apparatus has been designed and is under fabrication at the chemistry workshops of the University of California, Berkeley. The pulsed molecular beam uses a slightly modified piezo pulsed valve to the one widely used. The maximum jet cooling that will result will significantly reduce the number of rotational states in the superposition and thus reduce effects such as rotational dephasing. The principal investigator's laboratory was the first to successfully implement high harmonics to perform ultrafast pump-probe photoelectron experiments. High order harmonic generation will be developed here to produce an ultrafast photoionization source to be used in both the Raman wavepacket and coherent control experiments.

Initial studies will involve using the Ti-Sapphire laser to Raman pump multimode vibrational wavepackets in the ground electronic state. Prototypical molecules to be studied will be $\mathrm{CCl}_{4}$ and other Chlorinated Methanes where several vibrational modes can be simultaneously excited with the broad bandwidth of the Ti-Sapphire laser. Jet cooling these samples or preparing a launch state with a diode laser will greatly reduce the number of rotational states accessed and therefore avoid effects such as rotational dephasing. By phase manipulating these laser pulses, effects such as mode selectivity and wavepacket localization on multidimensional potential energy surfaces can be investigated. The vibrational coherences will be probed using vacuum ultraviolet ionization, greatly improving the detection sensitivity.

Recent theoretical work by Cina et. al. suggests that phase locked sequences of optical pulses are a powerful tool for measuring and mapping out non-stationary nuclear wavefunctions on electronically excited states. The next generation of experiments to be carried out here will be to adapt this approach to multimode Raman wavepackets in the ground state with the goal of mapping out the wavepacket on a multidimensional potential in real time. 


\section{Photoionization and Photoelectron Spectroscopy of He Droplets}

Principal Investigators: Daniel Neumark

Project No.: 01011

\section{Development of Highly Selective Catalysts in Supramolecular Nanoscale Reaction Vessels}

Principal Investigators: Kenneth Raymond and Robert Bergman

Project No.: 02011

\section{Project Description}

Homogeneous catalysis is of major economic importance to the energy and chemical industries. With many of today's catalysts though, a variety of problems exists, such as rapid catalyst decomposition, the need to use toxic organic solvents for the catalytic transformations, low substrate selectivity and in stereoselective reactions low enantio- or diastereoselectivities.

We have in hand a number of self-assembled, cage-like host molecules built through application of rational design. These assemblies are homoconfigurational with respect to the metal centers, and we are able to resolve these two configurations ( $\Delta \Delta \Delta \Delta$ and $\Lambda \Lambda \Lambda \Lambda$ ), which are then indefinitely stable to rearrangement. The ability of these container molecules to encapsulate, and even stabilize, a variety of monocationic molecules in aqueous solution is well established.

The major focus of this effort is to encapsulate cationic organometallic complexes that are known to be catalytically active, with the goal of carrying out highly substrate specific and stereoselective organic transformations within the assembled cavity. The use of these reaction "nanovessels" will allow non-aqueous catalytic transformations to be carried out in aqueous solution. Stereoselectivity may be achieved by utilizing the cavities imposed chiratity on the achiral catalyst and the substrates rather than working with chiral catalysts.

\section{Accomplishments}

A large variety of monocationic organometallic complexes has successfully been encapsulated into the cavity of the Ga4L6 tetrahedral assembly. If the organometallic species is water soluble, encapsulation can be achieved through simple displacement of the weakly bound NMe4+ cation in aqueous solution. Non water-soluble guest molecules can be included into the hydrophobic cavity under biphasic reaction conditions through a phase transfer exchange procedure. All of these reactions proceed within one hour. 
It appears that the specific, chiral environment of the cavity has a large impact on the encapsulated guest. Interestingly, the encapsulation of the planar chiral complexes of the general formula [CpRu(diene) $]+$ (diene $=$ isoprene, 2ethylbutadiene, 2-propylbutadiene, 2-isopropylbutadiene) into the chiral assembly leads to the formation of diastereomers in about a 1:1 ratio. This ratio can be changed if the sterically more demanding $\mathrm{Cp}^{*-\text {-complexes }}$ ( $\mathrm{CP}^{*}=$ pentamethylcyclopentadienyl) are used as guest molecules. Diastereomeric excesses of up to $70 \%$ are observed, illustrating that chiral recognition by the host cavity occurs if the cation is large enough.

In addition, iridium complexes of the general formula $\left[\left(\mathrm{CP}^{*}\right)\left(\mathrm{PMe}_{3}\right) \mathrm{Ir}\left(\mathrm{CH}_{3}\right)\left(\mathrm{C}_{2} \mathrm{H}_{4}\right)\right]^{+}$have also been encapsulated in the host assembly. At elevated temperatures, this complex reacts to give the decarbonylation of aldehydes. This reactivity has been shown to be affected by the chiral assembly. Diastereomeric ratios of the final product in excesses of up to $90 \%$ are observed. In addition, the host also provides steric selectivity, with the iridium complex being inert to larger aldehydes.

We now wish to investigate the possible reaction chemistry that can be conducted inside this assembly and eventually expand our research to larger sized nanovessels.

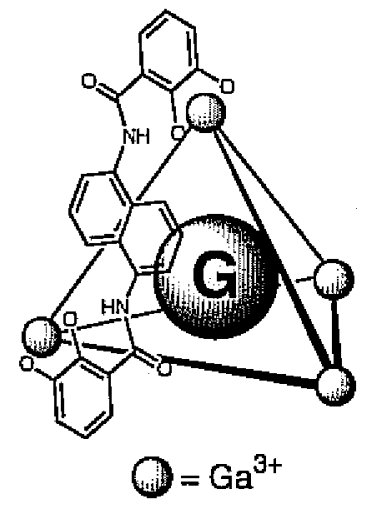

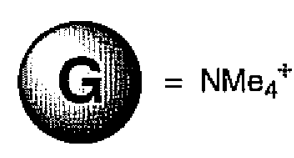

1

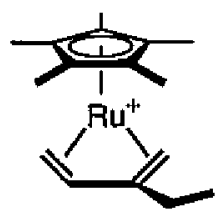

2

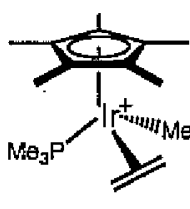

3
Figure 3: Supramolecular encapsulation of catalytic guest cations.

\section{Scientific Investigations and Technique Development of Wet Spectroscopy, High Pressure Photoelectron Spectroscopy, and STXM for Molecular Environmental Science}

Principal Investigators: David Shuh

Project No.: 02044

\section{Project Description}

Three end stations at the Advanced Light Source are the wet spectroscopy, high pressure photoelectron. spectroscopy, and the scanning transmission $x$-ray microscope (STXM) systems, respectively. The external experimental chambers are the soft $\mathrm{x}$-ray end station for environmental research, a wet spectroscopy surface science system, and a microjet system for the investigation of liquid surfaces. One common experimental theme and capability of all the end stations that we will apply is the ability to perform investigations of interfacial systems in the presence of water or under high pressure gas environments.

Research topics to be investigated are broadly defined in a range of scientific areas that include surface science, materials chemistry, interaction of biomaterials and organics at interfaces, and catalysis. A new potential area for study is the investigation the interfacial chemistry of manganese oxide materials with several contaminant and gas phase species. Investigation of this materials system would have substantial impact in the catalysis field. Similarly, there are numerous opportunities to investigate the interfacial chemistry of several gas phase and liquid species, including water and small molecules, at the surfaces of metal oxides and sulfides.

\section{Accomplishments}

A primary focus has been the scientific development and fabrication of end stations for use on the Advanced Light Source Molecular Environmental Science Beamline. This includes the two facility end stations, the Wet Spectroscopy and High Pressure Photoelectron Spectroscopy systems. Each has a hemispherical analyzer for electron detection and the former also has an $\mathrm{x}$-ray emission spectrometer. These end stations have been assembled, undergone 
preliminary testing, and have been mounted on the rotating, experimental platform of the beamline. Beamline commissioning was initiated and beamline development will continue for the next few months. The initial monochromator alignment and energy calibration have been performed.

A new, collaborative effort involves moving a liquid jet spectroscopy experiment onto the ALS-MES beamline for greatly improved investigations of liquid interfaces. This has necessitated the full redesign of the interaction chamber, the differential pumping section, and a host of other considerations to meet the experimental objectives made possible by the spectral characteristics of the ALSMES beamline. These developmental efforts will allow an improved set of experiments to be performed in the future. Another new set of experiments that is being explored will study aerosols and the reactions of aerosols in collaboration with the Chemical Dynamics Beamline. The Chemical Dynamics Beamline is developing an end station for the study of aerosols and a suite of experiments involving higher photon energies is ideal for the ALS-MES beamline. Finally, the novel use of the scanning transmission $x$-ray microscope (STXM) to characterize the properties of polymeric photoresistive materials with collaborators from IBM is being considered and evaluated.

During this initial period, considerable progress has been made establishing a modicum of research capabilities in a nearby laboratory and in the experimental area of the beamline, including implementing state-of-the-art researchbased gas handling manifold systems at the beamline for all end stations. 



\title{
Computing Sciences
}

\author{
(National Energy Research Scientific Computing Center, Mathematics Department, \\ Computational Research Division and Information Technologies and Services Division)
}

\section{The Experimental Mathematician's Toolkit}

Principal Investigators: David Bailey and Xlaoye Li

Project No.: 02012

\section{Project Description}

This project will develop a "toolkit" for doing experimental mathematics. This capability, which will be hosted at the National Energy Research Scientific Computing (NERSC)

Center, will enable a much broader range of mathematicians, physicists, and other researchers to utilize these emerging tools. This toolkit will be developed in collaboration with researchers at Simon Fraser University in Canada. During the past years, numerous computer programs for computational mathematics have been developed by the Principal Investigator and others. These include facilities for high precision arithmetic, integer relation detection, high-precision numerical quadrature, and automatic constant recognition. These programs however require specialized expertise to use, including expertise in Fortran-90, C++, message passing interface (MPI) paralleI programming, and numerical analysis. We will polish some of the prototype software now available, so that they are much more robust and do not require highly technical adjustments to obtain meaningful results. We will then encapsulate these programs into a highly usable interface, hosted on NERSC Center computers, accessible via the world wide web.

\section{Accomplishments}

During the past year, we have completed the Arbitrary Precision (APREC) computation package and teleased it for use by other researchers via the Internet. This software permits one to perform scientific computations with an arbitrarily high level of numeric precision, ranging from 100 digits to many thousands of digits as needed. This software package is written in $\mathrm{C}++$ for portability and efficiency, but it includes $\mathrm{C}+$ and Fortran-90 translation modules, which permit ordinary existing computer programs written in either of these languages to utilize the high-precision library routines by making only very minor changes to existing source code. The package includes the usual arithmetic operations, but also many transcendental functions, such as log, exp, sqrt, as well as integer relation detection and numerical integration.

We also completed a prototype of the Experimental Mathematician's Toolkit, based on the ARPREC package mentioned in item \#1. It permits one to perform many of these high-precision computations using an interactive tool, without even needing to write a computer program. One enters expressions in a Mathematica-like syntax, and the indicated computations are performed interactively using a high level of numeric precision, which can be set from 100 to 1000 decimal digit accuracy. Ordinary arithmetic expressions are recognized, together with many well-known constants and transcendental functions. PSLQ and numierical integration operations can also be performed.

Some specific scientific findings of our papers include: (a) the binary digits of pi and numerous other impottant constants of mathematics can be very accurately generated by means of simple recursive pseudorandom number generators; (b) several sequences have sums given by simple mathematical expressions; (c) the definite integrals of several functions are given by simple mathematical expressions; and (d) bound state spectra can be calculated very accurately using the ARPEC software, allowing Coulomb three-body systems to be effectively studied numerically rather than experimentally.

We gave numerous talks on various aspects of this work: (a) LBNL Summer Lecture, (b) UCLA mathematics colloquium, (c) U.C. Berkeley number theory seminar, (d) U.C. Berkeley numerical analysis seminar, (e) University of Maryland mathematics colloquium, (f) Institute for Defense Analysis, Center for Communications Research West (San Diego), and Sun Microsystems (Mountain View). The work on the normality of pi and related mathematical results continues to attract worldwide attention. In addition to articles in Science, Science News, Nature Online and Neue Zurcher Zeitung (Zurich) that appeared during FY2001, during FY2002 articles have appeared in BBC World News (London) and Investia (Moscow). Press coverage and follow-up articles in scientific journals have continued in FY2003. 


\section{Publications}

D.H. Bailey and R E. Crandall, "Random Generators and Normal Numbers," Experinental Mathematics, accepted for publication, March 2002. www.nersc.gov/-dhbailey/dhbpapers/bcnormal.pdf

D.H Bailey and D.J. Rudolph, "An Ergodic Proof that Rational Times Normal is Normal," LBNL Report 51142 , February 2002.

www.nersc.gov/-dhbailey/dhbpapers/ratxnormal.pdf

D.H. Bailey, D. Broadhurst, Y, Hida, X.S. Li and B. Thompson, "High Performance Computing Meets Experimental Mathematics," Accepted for publication. Proceedings of SC2002, September 2002. www.nersc.gov/ $\sim$ dhbailey/dhbpapers/expmath-sc2002.pdf

D.H. Bailey, "A Reclusive Kind of Science," Computing in Science and Engineering, August 2002. www.nersc.gov/ -dhbailey/dhbpapers/dhb-wolfram.pdf

D.H. Bailey and A.M. Frolov, "Advanced Variational Approach for High-Precision Bound-State Calculations in Three-Body Systems," Joumal of Physics B: Atomic, Molectular and Optical Physics, accepted for publication June 2002.

Www.nersc.gov/-dhbailey/dhbpapers/accura.pdf

D.H. Builey, Y. Hida, X.S. Li and B. Thompson, "ARPREC: An Arbitrary Precision Computation Package," IEEE Conference on Computer Arithmetic, submitted for publication, September 2002. www.nersc.gov/ dhbailey/dhbpapers/arprec.pdf

\section{Nonlinear Mathematical Models of Phenomena Related to Petroleum, Mining and Geological Engineering}

Principal Investigator: Grigory Barenblatt

Project No:: 01012

\section{Project Description}

The overarching purpose of this project is to study the mathematical models of the flow of complex fluids in complicated collectors. This study can be of importance for evaluation of the safety and reliability of proposed nuclear waste disposal schemes. More specifically, we will study of the fluid flows in diatomite rocks forming collectors of basic oil fields in California (Lost Hills, Belridge). A set of asymptotic and numerical methods will be used for solving the equations of non-equilibrium two phase flows in porous and fissurized-porous media and damage accumulation in aggressive environments and fissurized rocks.

\section{Accomplishments}

\section{Diffusion of $\mathrm{CO}_{2}$ in a Gas-filled Porous Medium with Immovable Water}

Diffusion of $\mathrm{CO}_{2}$ plays an important role in subsurface sequestration of this greenhouse gas, tertiary oil recovery by miscible and immiscible $\mathrm{CO}_{2}$ flooding, and in underground gas storage. First, we consider a simplified case of isothermal, countercurtent vertical diffusion of carbon dioxide and methane in a horizontally homogeneous reservoir. Initially, the bottom half of a uniform, homogeneous rock with no flow boundaries at the top and bottom is filled with $\mathrm{CO}_{2}$ and the upper half with $\mathrm{CH}_{4}$. Both gases are treated as ideal. At time equal zero, the two gases are allowed to diffuse. Under these restrictive assumptions, we obtain the exact solution to the initial and boundaryvalue problem using Fourier series method.

Second, we obtain a solution to the same initial and boundary-value problem in the integral form. The latter solution has a particularly simple structure and provides a good approximation of the exact solution. Its simplicity allows one to perform calculations that are tedious using the full Fourier serjes solution. The integral solution retains the important features of the exact solution and it can be used to test numerical algorithms.

Third, we consider a nonlinear diffusion problem admitting a self-similar solution. The latter solution provides a good approximation to the exact one before the diffusing gases reach considerable concentrations at the boundaries of the reservoir.

Fourth, we consider diffusion of $\mathrm{CO}_{2}$ with partitioning into connate water. We show that that at elevated pressures, the $\mathrm{CO}_{2}$ retardation by water cannot be neglected. The diffusion-retardation problem is reduced to a non-linear diffusion equation whose self-similar solution is found.

\section{Nonequilibrium Co- and Countercurrent Imbibition}

Spontaneous water imbibition is a complex process whose understanding is far from complete. It plays a crucial role in transport of soluble contaminants, including radionuclides from a leaking nuclear storage facility. In the oil industry, imbibition proceeds by water squeezing oil out of the rock matrix into a network of natural fractures or water-swept layers. We propose a mathematical model of the nonequilibrium counter-current imbibition that invokes two kinds of water saturation: time-dependent instantaneous 
saturation evaluated at each point of the block of a porous medium, and "effective" saturation, which is introduced to account for non-equilibrium effects. The model also includes a characteristic relaxation time, which is a nonlinear function of the saturation. We obtain a selfsimilar solution to the problem, which is valid at early times. We also perform scaling analysis of water saturation profile near the tip of the propagating front.

The investigation is in progress in FYO2 and will be continued in FY03. A working model has been developed; first analytic solutions have been obtained. The comparison of these solutions with experimental data has shown an instructive agreement.

\section{Dependence of Relaxation Time in the Non- equilibrium Imbibition on Water Saturation and Capillary Number}

Horizontal imbibition can be constructed in terms of two dimensionless numbers: the viscosity ratio and capillary number, and two descriptors of pore and flow geometry, e.g., the average ratio of radit of pore bodies and pore throats (aspect ratio), and the presence or absence of continuous water filaments in pore corners. The rock-fluid interactions can be quantified with the average hysteresis between the advancing and receding contact angle.

From mercury porosimetry it has been established that the advance of main terminal menisci is a complex dynamic process which does not attain equitibrium for very long times. The menisci are metastable and may relax to stable positions for several hours. Here we attempt to quantify the saturation relaxation time in non-equilibrium imbibition near the end-points of water saturation: vicinity of connate water saturation and residual oil saturation.

We find that close to connate water saturation the water-oil meniscus redistribution time should scale as with the proportionality constant depending on the medium permeability and applied pressure gradient. Near the residual oil saturation, the redistribution time goes to infinity for low capillary numbers.

\section{Does the KPZ Equation Model Dynamics of Moving Water-Air Interfaces?}

We demonstrate that the combined mass and momentum balances of water imbibing into a porous medium filled with air, whose one-dimensional version is known as the Richards equation, is the correct predecessor of the unrealistic Kardar-Parisi-Zhang (KPZ) equation used by many physicists to scale the dynamics of water imbibition. We show that the KPZ equation is insufficient to describe water imbibition, especially when gravity is involved. Consequently the interface-scaling relationships based on the KPZ equation, cannot reflect the true dynamics of water-air interface. This statement is only amplified in vertical water imbibition where an additional gradient term should appear in the governing equation.

We invert the governing Richards equation in one, two and three dimensions into a nonlinear partial differential equation governing the propagation of an interface associated with a fixed value of capillary pressure. This novel equation is then scaled to obtain self-similarity solutions.

\section{Investigation of Fluid Flow in Low-permeability Fragile Porous Rocks: A New Challenge for Subterranean Mechanics}

We face nowadays a new period in the development of subterranean mechanics: the science of flow, deformation and fracture in natural rock-fluid systems. Important practical problems with oil and gas recovery, water supply and, more recently, with the disposal of nuclear and chemical wastes, are forcing researchers to reconsider and modify the established theories of fluid flow, which dominated earth sciences with relatively minor changes since the late thitties.

To describe the fluid flow in fragile, prone to damage, and practically impermeable in pristine state rocks, we use a fundamental approach that appeared in the last four decades. In this approach, the microstructural properties of the material, directly observed or computed from the fundamental conservation laws, are explicitly introduced as a part of the model. In particular, the damage parameter understood as the average fraction of broken bonds in the rock is considered explicitly. The macroscopic fluid flow equations and the equations governing the kinetics of microstructural rock transformation are solved simultaneously. This micromechanical approach is the essence of our work on the flow and damage in fragile rocks.

We have obtained the first version of the mathematical model of the flow in the diatomites.

\section{Publications}

T. W. Patzek, D. B. Silin, S. M. Benson and G. I. Barenblatt, "On Vertical Diffusion of Gases in a Horizontal Reservoir" LBNL_48909, Journal Transport in Porous Media, in press.

G. I. Barenblatt, T. W. Patzek, and D.B.Silin, "The Mathematical Model of Non-equilibrium Effects in Wateroil Displacement," LBNL-49484, Society of Petroleam Engineers (SPE) paper \#SPE75169, April 2002. 
D. B. Silin and T. W. Patzek, "Spontaneous Countercurrent Imbibition: Two Asymptotic Solutions," Journal Transport in Porous Media, submitted for publication.

\section{G. I. Barenblatt, T. W. Patzek, D. B. Silin and V. M.} Prostokishin, "Oil Deposits in Diatomics: A New Challenge for Subterranean Mechanics," LBNL-49509, has been published as SPE paper SPE75230, April 2002.

\section{Infrastructure for Improving Protein Structure Prediction in Computational Biology}

Principal Investigators: E. Wes Bethel, Silvia Crivelli, Oliver Kreylos, Nelson Max, and Bernd Hamann

Project No.: 02013

\section{Project Description}

This project is to create a software-based environment for improving protein structure prediction in computational biology. The environment consists of high-performance protein structure optimization code coupled with disciplinecentric visualization and human interface technology. We will enhance an existing computational code developed at Berkeley Lab by creating a visualization software tool that makes it possible to generate a better set of initial conditions for the optimization and solver code, as well as to better understand the results and analyze different energy functions. Successful completion of the proposed work will result in: (1) a high-performance code for computing the tertiary structure of a protein; (2) a visual front end that can be used to create an initial condition for the optimization code with a graphical user interface (GUT) for human specification of biologically plausible initial configurations; (3) visual display of computed energy and locally minimized energy of a protein.

Our effort focuses on four primary areas: (1) making improvements to the computational engine that performs global energy optimization; (2) increasing the throughput of the local minimizations by parallelization of the local minimization algorithm and parallelization of the energy evaluation functions; (3) performing development of a visual front-end software tool that is used for display of geometry and energy of tertiary structures; (4) coupling these software components and deploying them in a way that promotes ease-of-use, and leveraging existing computational resources at the National Energy Research Scientific Computing (NERSC) Center.

Our first-year effort has focused on the design of a software infrastructure for improving the efficiency of the setup phase and dealing with complex structures. We have created a visual tool to quickly generate and directly manipulate protein structures. The tool forms $\alpha$-helices and $\beta$-strands based on ideal geometric definitions of the two types of local secondary structure, and then manipulates those structures to form tertiary structure. Interactive display of the energy value associated with the structure guides the selection of the best configurations for the next phase.

\section{Accomplishments}

The overall objective of the setup phase is to create initial protein configurations that are subsequently used as input to the optimization phase. Previous to our work, the setup phase was time consuming because it built initial configurations through expensive local minimizations with soft constraints. These soft constraints, derived from secondary structure predictions servers, encouraged the formation of $\alpha$-helices and $\beta$-sheets through the use of penalty (reward) functions. Two biasing functions were used to encourage formation of $\alpha$-helices.

In our work, we streamlined the process of creating initial configurations using a combination of a build up procedure that creates the protein structures by adding one amino acid at a time and an inverse kinematics procedure that easily manipulates those structures. This new setup phase allowed us to generate configuration at a much greater rate than before, and attempt to solve much larger problems than before. The new setup phase is depicted in Figure 1. It begins with a secondary structure prediction file and it is composed of two modules. The geometry generator module is used to ensily build $\alpha$-helices and $\beta$-strand structures and the interactive manipulator module is used to bring $\beta$ strands together to form $\beta$-sheets. The geometry generation module creates a "pre-configuration" consisting of fully formed secondary structures, but not tertiary structure.

We have tested our tool in the recent CASP5, a competition in which groups from around the world submit blind predictions of protein structures. Its use was instrumental in our CASP5 participation as it allowed for the fast creation of a diverse population of initial configurations regardless of the size and topology of the targets. The tool allowed our group to submit predictions of 20 targets considered to be a new fold or a difficult fold recognition target by the CAFASP servers. These targets ranged in size from 53 to 417 amino acids and contained more than ten pieces of $\beta$ strands. The tool was highly regarded by the community of 
biologists that recently gathered in Asilomar for the CASP5 meeting.

Recently, we coupled the interactive manipulation tool with the energy calculation code in order to perform energy visualization simultaneous with manipulation. This combination will allow for better initial configurations that have lower energy, and are more likely to converge. Next, we plan to couple the interactive manipulation tool with the Iocal energy minimization code to achieve better initial configuration (software-assisted design). In addition, we plan to change the energy code to improve local energy calculation by constraining local minimization computation to coil regions, which are those that change most during interactive manipulation. This will allow for faster energy computation by considering only the terms that change when the coil atoms move.

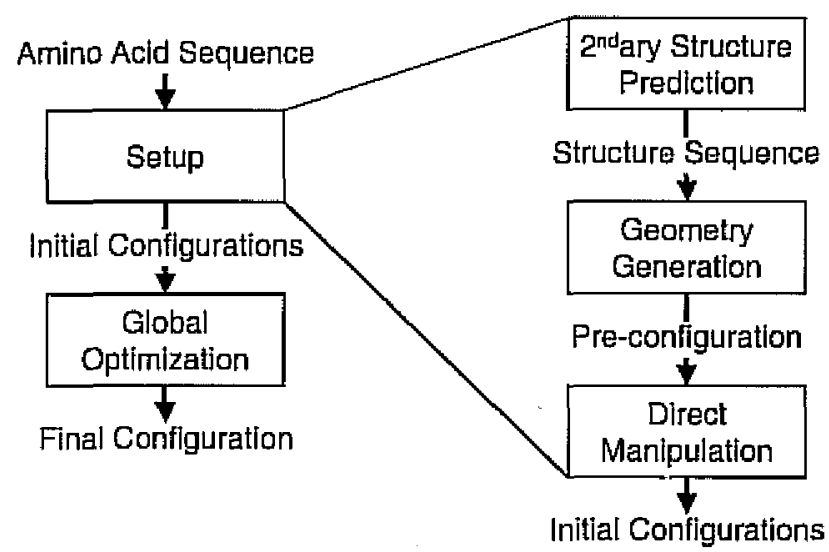

Figure I: Block diagram of protein configuration and optimization.

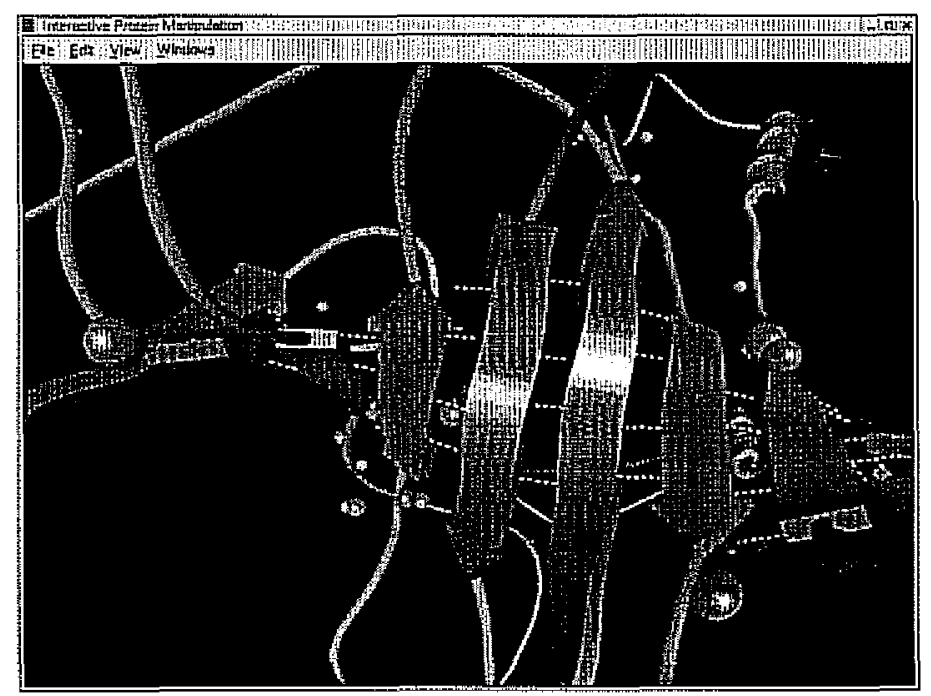

Figure 2: Creating beta sheets with the Interactive Visualization Software.

\section{New Machine Learning and Data Mining Methods for Genomics and Information Retrieval}

\author{
Principal Investigators: Chris Ding \\ Project No.: 02014
}

\section{Project Description}

Organizing observed data into groups or clusters is the first step in discovering coherent patterns and useful structures. This data clustering process is frequently encountered in scientific and engineering research. The goal of this project is to develop new and effective data clustering methods to discover unknown patterns in genomics and text datusets.

Existing clustering methods, such as hierarchical clustering method widely used in genomics applications, are often ad hoc by nature. We take a principled approach and introduce a MinMax clustering principle to minimize the overlaps between clusters and maximize the cluster self-similarities. Such an objective function optimization approach cast data clustering into a clear mathematical framework so that wellknown mathematical analysis can be effectively adapted. We develop this approach into a comprehensive cluster analysis framework, and apply to computational genomics and information retrieval problems. We study the critical issues in hierarchical clustering, cluster merging and splitting, and the dimension reductions widely used in data mining problems.

DNA micro-array gene expression technology revolutionalizes genomic research. A single micro-array chip can track thousands of genes of tissue samples, monitoring their pathological states. New technologies are being developed to analyze the gene expression profiles. We apply the clustering methods to these microarray gene profiles. Another explosive growth area is information in text form. Effective methods must be developed for efficiently sweep through the document databases. We develop methods taking into account the sparse representations of text information, viewed in vector space model.

\section{Accomplishments}

We have made a number of significant steps in cluster analysis of DNA micro-array gene expression profiles. We developed MinMaxCut algorithm for clustering tissue samples of Lymphoma produced by a Stanford group. The algorithm automatically discovers phenotypes using 
information solely embedded in the data. The results match human expertise by $92 \%$. Our work also show the usefulness of principal component analysis, a linear transformation based on singular value decomposition (SVD). Furthermore, we also devised a distance-sensitive method for ordering the genes in profiles, such that adjacent genes are similar while genes far-away in the order are dissimilar. This ordering method can also preserve the cluster structure. Several quantitative measures verify the effectiveness of the method. The ordering automatically and clearly exhibits interesting patterns in the expression profiles. Biological and clinical studies are often performed in the context of this order. These results are presented in RECOMB 2002 (the top conference in computational biology).

Selecting "marker genes" which correlates strongly with phenotypes is another important issue in micro-array gene expression profiles analysis. Standard methods make use of known phenotype information. We developed an unsupervised method that does rely on such prior knowledge. The method automatically discards "irrelevant" genes based on a two-ordering. This approach provides a new direction in gene selection.

We also developed a new framework for cluster analysis, based on the scaled principal components. The key feature of this framework is a new phenomenon we call as "selfaggregation", i.e., members of the same cluster aggregate automatically in this space. This feature and a number of theorems are proved vigorously using a perturbation analysis methodology borrowed from theoretical physics.

One of the most widely used methods in data mining is hierarchical clustering ( $\mathrm{HC}$ ). Using objective function optimization approach, we provide a comprehensive study of the key issues in $\mathrm{HC}$ : cluster merging and split, the selection methods and linkage function. Based on these studies, a new variant of HC is proposed and shown to be very effective.

Finally, most practical problems are of high dimension which are difficult to deal with (so-called "curse of dimension"). Dimension reduction methods are therefore very widely adapted in data mining. However, most if not all existing methods perform dimension reduction as data preprocessing. But the subspace obtained in preprocessing are not always the optimal subspace. We developed an adaptive dimension reduction clustering method that performs dimension reduction based on current clustering results, to a more suitable subspace. The subspace is gradually and adaptively updated towards the optimal subspace.
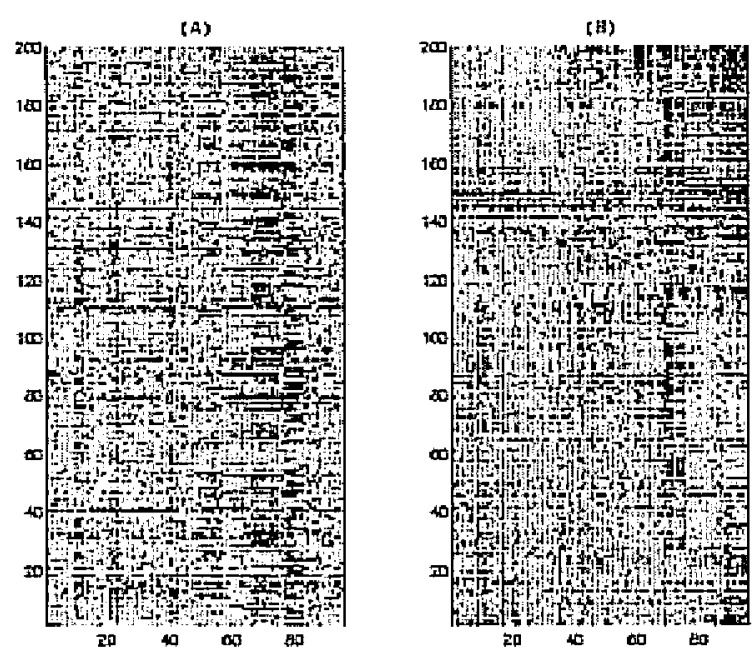

Figure 3: Optimal ordering of gene expression profiles of lymphoma dataset by Alizadeh et al. (Stanford U). Shown are 200 genes (rows) on 88 tissute samples (columns). (A) Data are displayed in the order provided by Alizadeh et al. (B) Both genes and tissues samples are ordered such that adjacent genes are similar while far-away genes are dissimilar. One can see clear pattems of low and high expressions.

\section{Publications}

C. Ding "Analysis of Gene Expression Profiles: Class Discovery and Leaf Ordering," Proc. $\sigma^{\text {th }}$ Int'l Conference on Computational Biology (RECOMB 2002), pp.127-136., April 2002.

C. Ding, "Unsupervised Feature Selection via Two-way Ordering in Gene Expression Analysis," Bioinformatics, April 2002, submitted for publication.

\section{Ding, $X . H e, H$. Zha, H.D. Simon, "Unsupervised} Learning: Self-aggregation in Scaled Principal Component Space," Proc. of Gth Eturopean Conference on Principles of Data Mining and Knowledge Discovery (PKDD 2002), pp. 112-124, August 2002, Helsinki, Finland.

C. Ding and X. He, "Cluster Merge and Split in Hierarchical Clustering," Proc. 2nd IEEE International Conference on Data Mining, pp. 146-153, December 2002, Maebashi, Japan.

C. Ding, X. He, H. Zha, H. Simon, "Adaptive Dimension Reduction for Clustering High Dimensional Data," Proc. 2nd IEEE International Conference Data Mining, pp. 154161, December 2002, Maebashi, Japan. 


\section{Numerical Simulation of Fuel Cells}

Principal Investigators: Joseph Grcar

Project No.: 02015

\section{Project Description}

The purpose of this project is to study the mathematical issues that arise in combining the most accurate models of fuel cell components and materials into a comprehensive, highly accurate computer simulation capability. The goal is to develop the numerical algorithms to bring the state-ofthe-art in fuel cell simulation to the high fidelity that will support more detailed fundamental studies of fuel cells for scientific or engineering design purposes.

The approach is to decompose the complex electro- and thermochemical system represented by a fuel cell into its constituent physical phenomena, and then to select or develop the numerical methods that are best suited to each component and to its interaction with the other components. This will result in simulation algorithms that are highly accurate, efficient, and yet robust with respect to extreme or unusual combinations of conditions in the underlying physical models.

\section{Accomplishments}

The first year's work has been devoted to acquiring an understanding of the range of physical models that have been developed to simulate fuel cells over the past decade.

Solid oxide fuel cells have been the primary focus because of their relative simplicity compared to other fuel cell technologies. Basically, fuel cell models can be grouped into three types: thin-film models, resistor-network models, and porous electrode flow models. The simplest, thin-filin models, are essentially phenomenological and treat the structural members of the fuel cell stack as circuit elements. The second, network models, add spatial parameterizations to the properties of the circuit elements, and represent the physical arrangement of the device's components through interconnected networks of the simpler models. The third type of model describes the cell's operation in terms of macroscopic conservation laws in the flow channels and porous membranes. The transport of reactants is modeled through the use of effective transport coefficients that take into account the volume fractions and tortuosities of the flow paths through the ceramic materials. These parameters can be estimated, and the chemical kinetics of many types of fuels are well known. As a result, these models are a suitable target for algorithm development. There remains a degree of uncertainty in the modeling of electrochemistry at the reactive interfaces, but this is being improved as a result of the experimental investigations. A reasonably complete theoretical model has been obtained from collaborating academic researchers. The mathematical algorithms needed to efficiently integrate the model's equations in 3-d simulations are being investigated. In addition, a preliminary survey has been made of similar models for polymer electrolyte fuel cells.

\section{High Performance System Area Networking for PC Clusters}

Principal Investigators: Paul Hargrove

Project No.: 02016

\section{Project Description}

At Berkeley Lab, across DOE, and throughout the scientific computing community, clusters of PCs built from commodity components are replacing traditional supercomputers. However, PC clusters can still benefit greatly from improved performance of the communications network. The network performance impacts many important areas: message passing [such as scientific applications based on message processing interface (MPI)], network I/O to and from a cluster, data storage and data staging, and specialized applications such as real-time visualization.

The InfiniBand Trade Association (ITBA) has specified InfiniBand ${ }^{S M}$, the next generation of commodity network. While targeted primarily at Enterprise Computing applications, we believe InfiniBand can greatly improve the performance of scientific applications on Linux clusters. This project is working as follows to ensure that InfiniBand will be a suitable message passing network in Linux clusters used for scientific computing, with an emphasis on MPL. In particular, we are testing and evaluating available InfiniBand hardware and software to understand how much of its theoretical performance InfiniBand cin deliver to applications.

\section{Accomplishments}

This project began with participation in the newly formed DAT Collaborative. This group formed largely from the memberships of the IBTA and the Virtual Interface Developers Forum (VIDF). On June 21, 2002 the DAT Collaborative ratified the $v 1.0$ specifications of the kernel- 
level and user-level Direct Access Provider Libraries (kDAPL and UDAPL). An initial Open Source reference implementation of uDAPL was released on September 9 , 2002.

The version 1.0 uDAPL APIs includes all of the functionality presently used in MPI implementations over VIPL, a similar API for hardware based on older the Virtual Interface Architecture. This is sufficient to access most of the features of InfiniBand that are valuable when implementing MPI. Implementations of MPI could benefit from additional features of InfiniBand, such as connectionless protocols. APIs covering these features have been deferred to the vl.1 specifications, anticipated in December 2002.

As a result of this project, L.BNL has signed nondisclosure agreements with most of the leading InfiniB and hardware and software vendors. Through the interactions we had with vendors it is evident that InfiniBand is not as mature as the industry had projected one year earlier. However, multiple vendors are developing very high-density compute servers based on InfiniBand backplanes. Such servers could make ideal building blocks for clusters used in scientific computing.

Installation of a test cluster to perform performance measurements was greatly delayed by vendors' slips in InfiniBand product cycles. However, we were able to complete a performance characterization of both $1 \mathrm{X}$ and $4 \mathrm{X}$ InfiniBand hardware. The results were not available in time for publication in FY02. The results suggest that $4 X$ IntiniBand is fully capable of delivering application-level bandwidth well in excess of the popular Myrinet and Quadrics networks. Small-message latency and software overhead are superior to those obtained with Myrinet 2000 on the same hardware. However, they are still slower than Quadrics timings obtained elsewhere. Software and firnware have not yet been optimized for performance. High CPU densities are achieved with blade-based designs which utilize an InfiniBand backplane for inter-processor communication. These designs deserve investigation as components for building Linux clusters used for scientific computing.

\section{Atomic Scale Modeling of Materials Chemistry}

\author{
Principal Investigators: Niels Jensen \\ Project No.: 01014
}

\section{Project Description}

Shock physics, materials chemistry, and non-equilibrium statistical mechanics are all integrated components in studying detonation of chemically active atomic-scale materials. The purpose of the proposed work is to model chemically active materials at the atomic scale and to answer fundamental questions such as: what reaction pathways are likely to exist in ' $n$ '-component reactions; shock-front speed and width; the detailed relationship between shock front and reaction front during the process; and the threshold values for sustained reaction propagation in different materials and structures with and without disorder. Our atomic-scale results can be compared directly to results of continum models describing chemical reactions at the macroscopic level.

This topic has wide interest in many scientific and industrial contexts, such as ion implantation of wafers where chemical reactions occur due to the deposited energy. We will apply lorge-scale molecular dynamics simulations of material composites consisting of several different types of atoms, all interacting with Brenner-Tersoff type potentials. The model material can be initiated in a number of different metastable states, after which a perturbation can provoke a sustained (or unsustained) chemical reaction of local structural atomic reorganization. This type of simulation is ideally suited for large-scale molecular dynamics, since we may easily require simulations of tens of millions of atoms, while the simulation time is limited by the fast timescales of active chemical reactions.

\section{Accomplishments}

We have investigated the effects of defects in explosive crystals on the initiation of a sustained reaction front. For the clissic two component model explosive, modeled by the Brenner type bond order potential, we have mapped the critical initiation shock strength necessary for ignition when an elliptical void is present at a given depth. The results show that the ignition is always lowered when defects are present and that defect position, size and orientation (if non-spherical) are strong factors in the initiation process. The study suggests that materials defects and disorder are 
critical components to understanding the behavior of high explosives.

We have further begun investigating the critical chemical parameters, such as energy release per molecule during chemical reaction, and molecular activation energy, for which sustained detonation can be obtained. This investigation provides a multi-dimensional phase-diagram of sustained detonation states as a function of molecular chemical reaction energy, activation energy, material dimension, molecular mass, molecular density, etc.

Finally, we are conducting detailed simulations to address the relationships between the model atomic scale explosive and corresponding continuum model descriptions. Particularly, boundary conditions at the initiation are currently being investigated for obtaining a direct connection to much of the known properties of continuum models of reactive materials.

\section{Publications}

T. Germann, B. Holian, P. Lomdahl, A. Heim, N. Gronbech-Jensen, J-B, Maillet, "Molecular Dynamics Simulations of Detonation in Defective Explosive Crystals," Proceedings from the $12^{\text {th }}$ Intemational Detonation Symposium, San Diego, CA, August 11-16, 2002, in press.

A. Heim, N. Gronbech-Jensen, T. German, B. Holian, P. Lomdahl, "Initiation and Probagation of Reaction Fronts in Chemically Active Materials," in preparation.

\section{Suitability of Alternative Architectures for Scientific Computing in the NERSC-5 Time Frame}

Principal Investigators: Xlaoye Ll, Leonid Oliker, and Katherine Yelick

Project No.: 01015

\section{Project Description}

As we look beyond NERSC-4, important questions arise regarding how key scientific applications will perform on future generation supercomputers. Currently any high performance applications run well below the peak arithmetic performance of the underlying machine, with inefficiencies often attributed to a lack of memory bandwidth. A new class of microprocessors designed to address the memory gap is emerging from several leading academic and industrial institutions. We plan to evaluate the feasibility of using large-scale multiprocessor systems based on this technology for key scientific computations.

To accomplish this, we will evaluate how key computational codes now in use at the National Energy Research Scientific Computing (NERSC) Center and the Department of Energy (DOE) will perform on future generation architectures. We will incorporate the novel media architectural research being developed at University of California (UC), Berkeley and Stanford University. In addition to porting codes onto prototype systems, we will use simulation and analytic modeling to predict the expected performance of these applications on large-scale systems. Based on detailed simulations and executions, we will modify the algorithms to better fit the architecture and improve the compiler and performance tools. We plan to isolate the set of application characteristics best suited for each architecture and study the potential of interfacing leading-edge media processor technology with high-end scientific computing.

\section{Accomplishments}

This work examined two emerging media microprocessors, VIRAM and Imagine, and evaluated their potential efficacy for addressing the growing memory-gap of high-end numerical simulation. The VIRAM of UC Berkeley is an example of a processor-in-memory (PIM) or intelligent random access memory (IRAM) design. A set of memoryintensive benchmarks where used to compare the performance of conventional cache-based microprocessors to the mixed logic and dynamic random access memory (DRAM) processor of VIRAM. Our experience with these benchmarks suggest that VIRAM is significantly faster than conventional processors for problems that are limited only by DRAM bandwidth and latency, and because VIRAM achieves high performance through parallelism tather than a fast clock rate, the advantages are even larger if one is interested in building a power-efficient multiprocessor system. Although we have concentrated on the memory systems within a single node, we believe these results indicate that IRAM would be a reasonable building block for large-scale multiprocessors. More work is needed to understand how well a system of IRAM processors would be balanced, given current networking technology.

We then compared VTRAM with the Imagine architecture. Imagine is a programmable streaming microprocessor currently being developed at Stanford University. Stream processors are designed for computationally intensive applications characterized by high data parallelism and producer-consumer locality with little global reuse. First, we developed a scalable synthetic probe that allowed us to parameterize key features of the architectures. By varying a 
small set of parameters we explored performance in the context of: computational intensity, vector/stream length, memory access patterns, kernet overheads, producerconsumer locality, and hierarchical memory structure. We then evaluated sparse matrix vector multiply (SPMV) and QR decomposition (QRD), two important scientific kernels with distinct program behavior, and demonstrated which architecture is best suited for a given class of computational requirements.

We also studied the complex interactions between programming paradigms, architectural support at the industry standard architecture (ISA) level and the underlying micoarchitecture of the VIRAM and Imagine systems. Some benchmarks were able to leverage the multiword record support of the streaming architecture. Although VIRAM's compiler was able to vectorize these multi-word codes, it was restricted to using the native vector instructions which only operate on basic-data types. As a result, VIRAM was forced to incur the overhead of strided memory accesses. However, program development was more challenging in Imagine than in the well-known vectorization paradigm, because the programmer is exposed to the memory hierarchy and cluster organization of the Imagine architecture. Improvement in the quality of the compiler and software development tools, and abstracting lower level details of the hardware will be essential in bringing the stream programming model to the wider scientific community. In this work we successfully demonstrated the overlap between emerging high-end media processors and scientific computations. We gained key insight into the salient features of VIRAM and Imagine in the context of numerical kernels, and quantified the computational space best suited for each processing paradigm.

\section{Publications}

M. Narayanan, L. Oliker A. Janin, P. Husbands, and X. Li, "Scientific Kernels on VIRAM and Imagine Media Processors," International Parallel \& Distributed Processing Symposium (IPDPS), submitted for publication in April 2003. http://www.nersc.gov/ oliker/papers/IPDPS_03.pdf

S. Chatterji, M. Narayanan, J. Duell, and L. Oliker, "Performance Evaluation of Two Emerging Media Processors: VIRAM and Imagine," Workshop on Parallel and Distributed Computing in Image Processing, Video Processing, and Multimedia (PVIDM), submitted for publication in April 2003.

B.Gaeke, P. Husbands, X. Li, L. Oliker, K. Yelick, and R. Biswas, "Memory-Intensive Benchmarks: IRAM vs. CacheBased Machines," International Parallel \& Distributed
Processing Symposium (IPDPS), April 2002. http://www.nersc,gov/-oliker/papers/ipdps02.pdf

\section{Segmentation of Mammary Gland Ductal Structure using Geometric Methods}

Principal Investigators: Ravj Malladi

Project No.: 00217

\section{Project Description}

The goal of this project is to develop image analysis algorithms for automatically detecting, segmenting, and visualizing three-dimensional tissue structure from series of two-dimensional histological sections. This requires highly optimized segmentation algorithms, able to perform massive computations in a short period of time. Such a unique capability will have a tremendous impact in the scientific community, opening a new avenue for adding the morphological parameter to the already existing molecular and cellular investigation. We aim to use the geometric Partial Differential Equation (PDE) methods, and the level set framework to build schemes both for image registration and for segmentation of mammary gland and ductal structures.

\section{Accomplishments}

We made progress on many fronts. First, in order to visualize large data sets and surfaces in motion, we built a Visualization tool kit (VTK) based visualization framework that works in tandem with our PDE solvers. This has been built to operate on both Linux and Solaris platforms.

Second, to increase the contrast in the images, we built a transform domain technique to eliminate the background squares that are often present in scanned sections. Without this step, it is very likely that the edges due to the 'grid' will interfere with the analysis.

The main thrust has been in perfecting the fast Additive Operator Split (AOS) methods for non-linear PDEs such as Beltrami flow and other reaction-diffusion equations. We now have a first order accurate, unconditionally stable, semi-implicit method to solve a host of PDEs both in two and three dimensional (2D and 3D). We are obtaining speed-up factors in the range 50-150 on typical images. 
This has drastically reduced our compute times on the image denoising step. As before, we are using a combination of marching methods (to move interfaces) and the level set geometric PDE methods to segment the ductal structure. We have built schemes to automatically 'filter out' certain structures as a post-processing step. The 2D segmentations are pieced together to realize a 3D structures; one such example is shown in the enclosed figure. According to our plan, we have started doing the calculations directly in 3D by artificially generating a 3D volume from a set of $2 \mathrm{D}$ images.

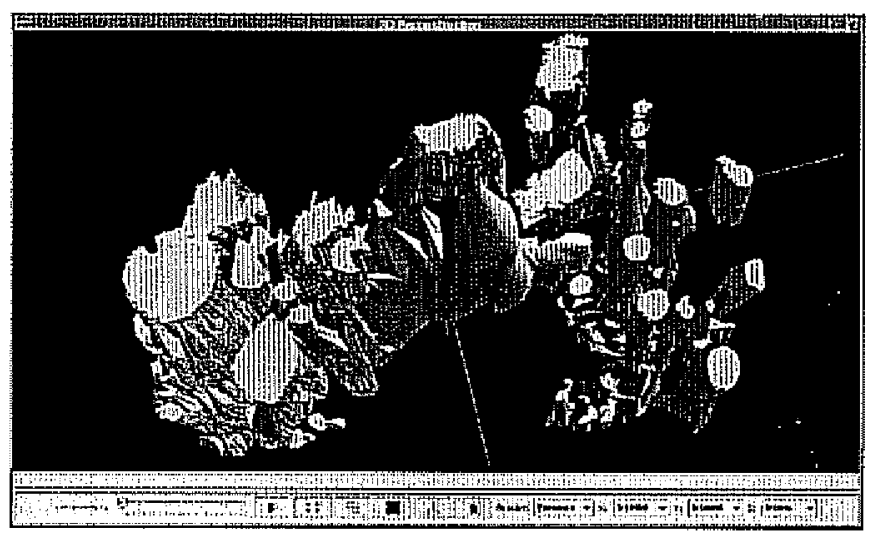

Figure 4: 3-D surface rendering of a human case. Tumor masses are represented as gray volumes.

\section{Publications}

R.F. Gonzales, U. Adiga, A. Idica, T. Deschamps, R. Malladi, and C.O. Solorzano, "Automatic Segmentation of Structures in Normal and Neoplastic Mammary Gland Tissue Sections," Proc. of SPIE, Vol. 4964, to be published January 2003.

T. Deschamps, R. Malladi, and I. Ravve, "Fast Evolution of Image Manifolds and Application to Filtering and Segmentation in 3D Medical Images," Special isaue of IEEE Transactions of Visualization and Computer Graphics, on Mathematical Visualization, October 2002, in press.

R. Malladi and I. Ravve, "Fast Difference Schemes for Edge-enhancing Beltrami Flow and Subjective Surfaces," Visualization and Mathematics, Ed. Hege \& Polthier, Springer-Verlag, September 2002.

\section{Parallel Methods for Robust Optimization and Uncertainty Quantification}

Principal Investigators: Juan Meza

Project No.: 02045

\section{Project Description}

Optimization of functions derived from the modeling and simulation of some physical process constitutes an important class of problems in many scientific applications. The main characteristic of these types of problems is that the function evaluation is computationally expensive and dominates the total cost of the optimization problem. It is desirable that the optimal solution be robust in the sense that small changes in the model parameters or data do not generate large perturbations in the optimal solution. This research will focus on two major areas: (1) the development of parallel algorithms for robust optimization under uncertainty and (2) the development of parallel methods for uncertainty quantification in simulation models.

We propose to focus on new methods that combine the parallel direct search method and the trust-region method into a new class of algorithms called Trust-Region Parallel Direct Search (TRPDS), which takes advantage of the best properties of both algorithms. TRPDS has been shown to have rapid convergence rates typical of Newton-type methods while gaining the advantage of parallelism inherent in the parallel direct search methods. This research will extend that earlier work to increase the robustness of TRPDS through the use of interior point methods. We also propose to investigate the use of filter methods for nonlinear constraints in the setting of interior point methods. As part of the uncertainty quantification problem, we will also develop methods for model reduction and surrogate functions.

\section{Accomplishments}

There were two major accomplishments in the initial period of this project, (1) the incorporation of a parallel genetic algorithm into the OPT+ software package and (2) the implementation of a new set of test problems for robust optimization. The new genetic algorithm (GA) is an example of a parallel derivative-free optimization method that is useful for solving nonlinear optimization problems where there are no derivatives available. Although the GA 
algorithm does not have rapid convergence properties, it is nonetheless useful in certain cases, for example where the optimization parameters are discrete. We envision this algorithm as a first step in the development of a suite of derivative-free optimization methods within the OPT + framework.

We are also currently working on the inclusion of 300 new test problems from the Schittkowski set of problems. These new test problems will allow us to rapidly compare the various new algorithms we have proposed across a wide range of problems. We are also developing a new test problem for robust optimization based on an inverse problem for computing the diffusion coefficients in a fluid flow problem. The inverse problem itself is modeled using the pVARDEN code for solving variable density incompressible Navier-Stokes problems. This model problem can be used to represent various problems of interest including combustion and fluid flow.

\section{Publications}

Presentations: ACTS (Advanced Computational Software) Workshop, NERSC/LBNL, September 2002.

\section{Second-order Methods for Solid-Fluid Shock Coupling with Application to Martian Meteorites}

Principal Investigators: Gregory Miller

Project No.: 01016

\section{Project Description}

We are developing new numerical methods do model impact problems that include multiple materials separated by complex time-dependent boundaries. Our goal is an accurate, robust solver that can be used for very large threedimensional problems by exploiting parallelism and adaptive mesh refinement.

We are applying our new methods to impact problems on planetary surfaces. The specific scientific application we are concerned with is the mechanism by which meteorites have been sent from Mars to the Earth, presumably by impact, but without showing evidence of having experienced large shock pressures. Two qualitative models have been suggested. In one, jets emitted in an impact propel rocks from the surface - like a fire hose sweeping pebbles off pavement. In the other, an interaction between the compressive shock and zero-pressure surface boundary condition is imagined to provided large accelerations by smalt pressures. We plan to model realistic scenarios with sufficient fidelity that the feasibility of these proposed mechanisms may be assessed.

The approach we adopt consists of three major components. First, we are developing a new numerical framework for solving multiphase dynarnics on Eulerian grids, with adaptivity, and in parallel. The second step consists of constructing new hyperelastic equations of state and constitutive models. This is necessury to accurately model the response of rocks and ice to high velocity impact. Two key concerns are phase change (melting and vaporization), and fracture (breakup of a solid into a rubble with no strength in tension). Third, there are adjustments to the numerical method that may improve its robustness and accuracy. This involves substantially new algorithm. development.

\section{Accomplishments}

As reported last year, we have developed an implementation of an Eulerian multimaterial schock capturing code in 3D. This code uses the Chombo toolkit (http://seesar.lbl.gov/anag/chombo/) to exploit parallelism and to implement adaptive mesh refinement. We are early adopters of the Chombo framework, and have provided feedback and error reports to guide its development.

With this new code we have continued to improve modeling of simple impact problems, as shown in the attached figure 5. This figure shows a snapshot in time of an initially spherical impactor having penetrated the surface of the target material. The initial trajectory was oblique at 45 degrees and $20 \mathrm{~km} / \mathrm{s}$ velocity. The materials are rock-like elastic-plastic solids. A preliminary analysis of this result, and of other similar computations, suggests a compelling mechanism for the Martian meteorite problem. The impactor flow approximately stagnates, and spreads radially relative to the impact vector. This flow propels target materials at high velocity in an approximately isentropic (shockless) manner. This mechanism, if correct, will be very important because it calls for no special geology or special impact characteristics: merely oblique impact of sufficient velocity. Work is underway to address certain deficiencies of our method that have cropped up in the high velocity impact calculations. The most pressing is an interplay with a new subgrid spall model (a mechanical constitutive model for material in tension) with the interface reconstruction and mass redistribution algorithm. To rectify this problem we are looking at dynamic free stream preservation mechanisms. In parallel with the development of our new numerical methods, this LDRD has supported a 
UC Davis graduate student who has been working to extend the capabilities of the ChomboVis visualization tool being developed by the ANAG group. The key features student Chris Coe has worked on concern the visualization of material interfaces described by a volume fraction field. Results of his work have been integrated into ChomboVis and were used to present our computational results at the 2002 Supercomputing meeting.

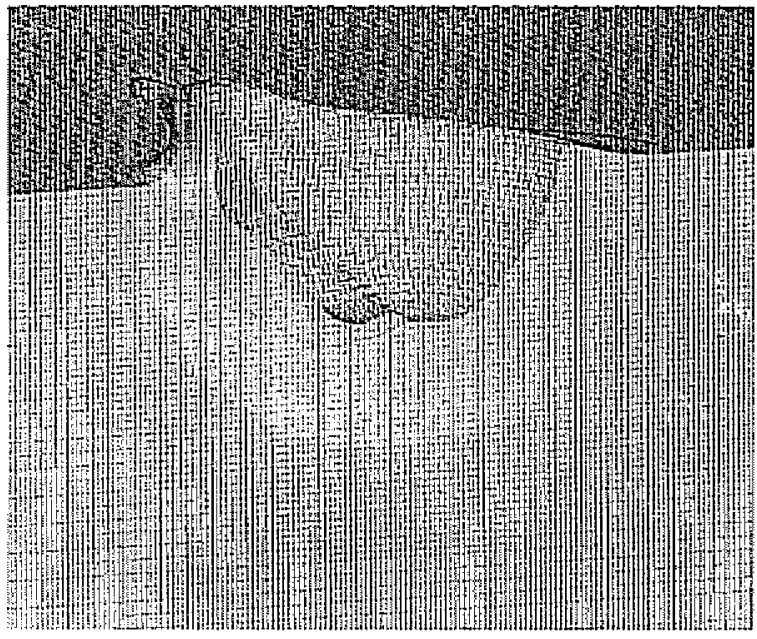

Figure 5: Material ejected from Mars in a 20kn/sec., 45 degree impact, via shockless stagnation-driven flow.

\section{Publications}

G.H. Miller and P. Colella, "A High-Order Eulerian Godunov Method for Elastic-Plastic Flow in Solids," J. Comp. Phys., 167, 131-176, (LBNL-45647), 2001.

G.H. Miller and P. Colella, "A Conservative ThreeDimensional Eulerian Method for Coupled Fluid-Solid Shock Capturing," J. Comp. Phys., (LBNL-50932) in press.

\section{Adaptive File Replication and Coordinated Transfer for Data Intensive Grid Applications}

\section{Principal Investigators: Ekow Otoo}

Project No.: 01017

\section{Project Description}

The objective of this work is to develop adaptive distributed algorithms that coordinate and compute optimal strategies for accessing required subsets from very large datasets on a data-grid. The envisioned environment is a high-speed network that interconnects hierarchical data storage systems, distributed disk caches and computational nodes. The datasets are maintained as collections of files and are distributed over some specialized nodes/sites that have tertiary storage. Computations make requests for files that are subsets of the datasets. The goal then is to develop an optimal strategy for caching and replicating files in distributed caches so that requests are processed efficiently over the network.

Related problems in this area are known to be NP-hard (an optimization problem on a particular complexity class of decision problems referred to as nondeterministic polynomial time algorithms), and hence our goal would be to strive for good heuristic algorithms. The algorithms to be developed would exploit knowledge of the network bandwidth, statistics of data access pattern and information from meta-data and replica catalogues to generate an optimal access plan for any applications' data requests. Based on the knowledge of availability of disk cache space, access pattern and the query classes, the algorithms determine policies for retaining and purging file replicas from the disk caches. The results of this research are applicable to other research projects in scientific computing that are ongoing at DOE and other similar agencies. Examples include: Particle Physics Data Grid (PPDG), Earth Science Data Grid (ESDG) and National Aeronautics and Space Administration's (NASA) Earth Observation System Data and Information System (EOSDIS). The work was extended to address:

- The use of real workloads from trace data of requests for datasets in scientific experiments such as Solenoidal Tracker at RHIC (STAR).

- Multi-tier caching of data files where the datasets resides on node with implied hierarchy designated as National, Regional and Local centers have different bandwidth and latency for data transfers and accesses.

- Prioritized control of file retention in the caches.

\section{Accomplishments}

The main accomplishments in this project include:

- The development of two new performance metrics for evaluating disk cache replacement policies termed the average cost of retrieval per reference (ACPR) and the cache availability respectively. Given a workload, ACPR is defined as the ratio of the total cost, in time, of all file accesses made into the cache to the number of successful references. The availability is defined as the percentage of references that were successfully satisfied by a caching policy for a specific cache size. The 
significance of the ACPR performance measure is that it is able to provide a good indication of how much cache size is required for a given traffic of file accesses at a particular site. In figure 6 below which shows the graph of ACPR for varying cache sizes of a workload generated at the National Energy Research Scientific Computing (NERSC) Center, the minimum cache size required to handle the file references for the caching activities monitored at NERSC Center is about 500 Gigabytes.

- The definition of a utility function $\phi_{i}(t)$ for determining the file to be replaced from the cache at time $t$, and an algorithm for computing a replacement in time $O\left(\log _{2} N\right)$ in a cache of $\mathrm{N}$ file objects. Given that a file $i$ in cache is of size $s_{j}$ and has a cost $c_{i}(t)$ of retrieving it into the cache. If $g_{i}(t)$ is the accumulated number of references to the file and the time of the $k_{\mathrm{f}}(t)$ backward reference is $t_{\text {-k }}(t)$, up to a maximum of $K$. Then the utility function is defined as

$$
\phi_{i}(t)=\frac{k_{i}(t)}{t-t_{-k}(t)} \times \frac{g_{i}(t) \times c_{i}(t)}{s_{i}(t)}
$$

The candidate for replacement at time $t$ is the file with the minimum value of $\phi_{i}(t)$

- The development of a discrete event simulator for estimating the time it takes to replicate, transfer, cache and process files at a specific site in a given topology of distributed data.

The algorithms and data structures that govern the service, caching and cache replacement policies are now being used in the redesign and implementation of a policy advisory module (PAM), as the intelligent component of storage resource managers of data grids under the Scientific Discovery through Advanced Computing's (SciDAC) Scientific Data Managenent (SDM) Center activities.

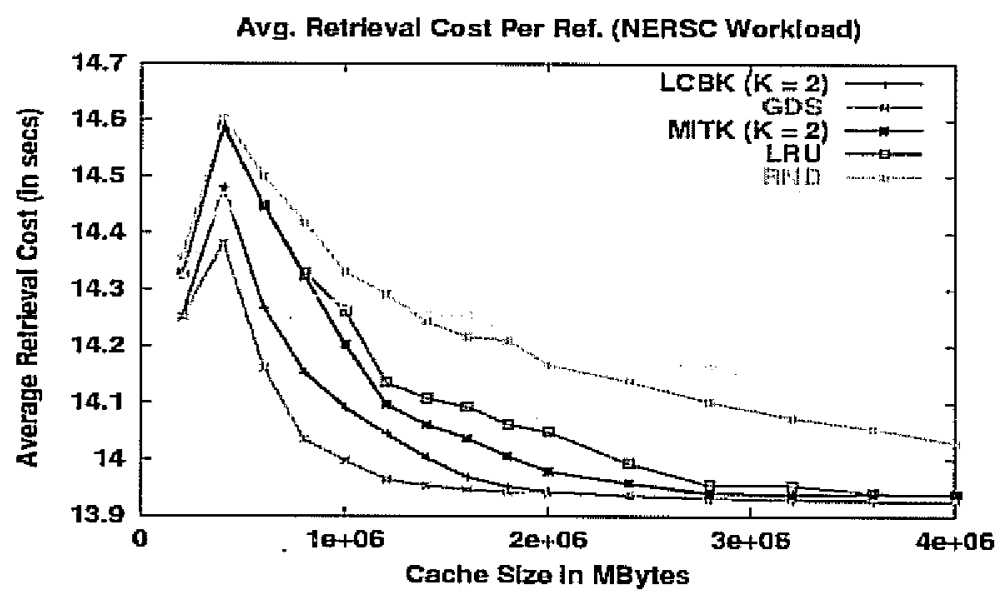

Figure 6: Graphs of average cost per retrieval for real workload from NERSC Center.

\section{Publications}

E.J. Otoo, F. Olken, and A. Shoshani., "Disk Cache Replacement Algorithm for Storage Resource Managers in Data Grids," The $15^{\text {th }}$ Annual Super Computer Conference, SC2002, Baltimore, Maryland, November 2002.

E.J. Otoo and A. Shoshant, "Accurate Modeling of Cache Replacement Policies in a Data Grid," 11 th NASA Goddard Conference on Mass Storage Systems and Technology/20 ${ }^{\text {th }}$ IEEE Symposium on Mass Storage Systems, San Diego, California, April 2003, to appear.
E.J. Otoo, D. Rotem, D. and A. Shoshani, Discrete Event Simulator of Adaptive File Replication and Coordinated Transfers in Data Grids, in preparation.

E.J. Otoo, D. Rotem, A. Shoshani and T. Moscovitz, The Architecture of a Disk Resource Manager Using a Policy Advisory Module: Design Doctment and Reference Mantal, in preparation. 


\section{Combinatorial Algorithms in Scientific Computing}

Principal Investigators: All Pinar

Project No.: 02018

\section{Project Description}

This project aims at applying combinatorial techniques to improve performance of computation-intensive applications. This makes the project inherently multidisciplinary: computer science and discrete mathematics on one hand and the applications on the other. Under this project, we are addressing this broad goal by studying several problems, which come from different fields and require different solution techniques.

Our efforts focus on three major fields: (1) parallel scientific computing, where we are investigating new load balancing techniques and parallel algorithms for some graph algorithms (e.g. strongly-connected components, graph matching), (2) data storage management, where we work on organizing a database to efficient indexing of scientific databases), and (3) computational biology, where we are looking at sequence alignment algorithms.

\section{Accomplishments}

\section{Parallel Scientific Computing}

In many applications of parallel computing, distribution of the data unambiguously implies distribution of work among processors. But there are exceptions where some tasks can be assigned to one of several processors without altering the total volume of communication. We studied the problem of exploiting this flexibility in assignment of tasks to improve load balance. We first modeled the problem in terms of network flow and use combinatorial techniques for its solution. We propose two algorithms based on parametric search and augmenting paths. We then considered a continuous version of the problem, and formulated it as a linearly constrained optimization problem, i.e., min $\|\mathrm{Ax}\|_{\infty}, \mathrm{s.t} \mathrm{Bx}=d$. 'To avoid solving a hard-to-solve $\infty$-norm optimization problem, we showed that in this case minimizing the 2-norm is sufficient to minimize the $\infty$-norm, which reduced the problem to the well-studied linearly-constrained least squares problem. The continuous version of the problem has the advantage of being easily amenable to parallelization.
Experimental results clearly demonstrate effectiveness of our techniques. In a molecular dynamics application, we were able to reduce $58 \%$ imbalance to only $2 \%$ for 512 processors. For applications where overlapped Schwartz domain decomposition is used, we were able to produce balanced workloads in all instances, where the initial load imbalance values were as bad as $138 \%$, which means one processor is loaded twice more than average load. Work for the following year will be based on investigating possible applications of the idea to large scale projects such as the Scientific Discovery through Advanced Computing (SciDAC) accelerator project.

\section{Data Storage Management}

In many scientific domains, experiments generate large volumes of data, to raise the problem of how to store terabytes of data such as billions of tuples with hundreds of properties and run queries efficiently on them. Properties are either categorized or continuous variables. What has been proposed by the Scientific Data Management Group at National Energy Research Scientific Computing (NERSC) Center is to divide ranges of continuous variables into bins, and locate each item in one bin. This enables storage of the information with only bits, so that we have a " 1 " in a bin if the entry falls into the corresponding range or is in that category. For efficiency of queries, the information in one bin is stored as a bit slice. Notice that it is sufficient to store only the "I"s for each slice. Moreover, for storage efficiency, these vectors are compressed such that for a sequence of consecutive " 1 "s, we store the index of the first one, and the sequence length.

Effectiveness of this technique relies on positions of the "1"s in each slice, so that performance increases when "1"s are in consecutive positions, thus reordering tuples of the database to align " 1 "s to consecutive positions will improve performance. The reordering problem can be stated as a version of the well-known traveling salesperson problem. However the size of the graph and specific information on the graphs open some room for research on this instance of the traveling salesperson problem. We tailored techniques for this intance of the "Traveling Salesperson Problem," and experiments show that significant reduction in storage size and query times can be achieved by reordering the tuples of a database.

\section{Computational Biology}

Alignment and comparison of very large sequences is u fundamental problem in bioinformatics. Although there are good algorithms for relatively short sequences, aligning very large sequences (e.g., whole chromosomes) requires further research. We are investigating how to use parallel machines to speed up these computations. 


\section{Publications}

A. Pinar and B. Hendrickson, "Exploiting Flexible Assignable Work to Improve Load Balance," Proceedings of I4th ACM Symposium on Parallel Algorithms and Architectures, pages: 155-163, August, 2002.

A. Pinar and C. Aykanat, "Fast Optimal Load Balancing Algorithms for 1D Partitioning," Journal of Parallel and Distributed Conputing, submitted for publication.

A. Pinar, T. Tao, and K. (John) Wu, "Efficient Indexing for Scientific Databases," in preparation.

\section{Scalable Methods for Studying Collisional Breakup and Rearrangement Processes}

\section{Principal Investigators: William McCurdy and Thomas Rescigno}

Project No.: 02019

\section{Project Description}

Sophisticated first-principles calculations of collisional ionization have so far been limited to electron impact on the simplest target, atomic hydrogen. By contrast, electron collisions with heavier, multi-electron atoms have been limited in terms of the level of accuracy of the underlying methodologies employed and the types of processes that have been studied. For the case of positron impact, there are currently no detailed studies of collisional ionization, even for the case of one-electron targets.

The purpose of this project is to develop scalable methods for studying collisional ionization that will be practical to apply to multi-electron targets. We will extend our studies to cover breakup in the presence of rearrangement channels, developing methods to treat collisional ionization by positron, as well as electron, impact.

\section{Accomplishments}

The exterior complex scaling (ECS) method is the foundation of our approach for studying collisional breakup. This method, which has produced the first essentially exact treatment of electron-impact ionization of atomic hydrogen, provides a path to the computation of wave functions from which dynamical information can be extracted without having to explicitly impose detailed scattering boundary conditions. During this past year, we have focused our efforts on extending and improving the basic methodology so that we can handle collisional ionization of targets with two active electrons. Since application of exterior complex scaling to the timeindependent Schroedinger equation leads to systems of linear equations whose dimensionality scales poorly with particle number, we have explored the feasibility of applying this method in the time domain, thereby avoiding the direct solution of complex linear equations. With the time-dependent formulation of ECS, the system wave function is rigorously computed by propagating a wave packet on an exterior-scaled grid and accumulating its Fourier transform during the course of propagation. The fensibility of the method was established by applying it to a variety of 2-dimensional problems, including the TemkinPoet model for electron-hydrogen ionization, and showing that we could reproduce the results obtained with the original time-independent treatment. We were also successful in applying the method to a model 3-dimensional problem involving short-ranged interaction potentials. These results were published in Physical Review A.

These early results using the time-dependent method were obtained by employing a version of the split operator approach and a simple Cranck-Nicolson propagator along with a combined finite-element/ discrete variable representation of the Hamiltonian. Application to realistic problems with long-range Coulomb interactions requires large grids and long propagation times. To accommodate these needs, and to improve numerical accuracy as well, we have turned to Krylov-based propagation methods in our implementation of time-dependent ECS. In our case, Krylov-based methods rely on expanding the time evolution operator in a small subspace obtained by repeated application of the Hamiltonian matrix to a vector. This procedure is fast, is ideally suited to parallel implementation and, by eliminating the need for the splitoperator approximation, gives us complete control over the error in the propagation. Using these techniques, we have begun to investigate the 3-dimensional S-wave model for electron-helium ionization. Our initial results for excitation and total ionization cross sections, which compared well with the only other published theoretical treatment of this problem, were presented at the annual DAMOP meeting of the American Physical Society. Our goal is to provide the first computational results for the differential ionization cross sections at low energy in the S-wave model, for which no results presently exist.

A major challenge in the development of these essentially 'exact' grid-based methods for computing scattering wave functions is a prescription for extracting the differential ionization cross sections which give detailed information about the-energies and angles of the ejected electrons. This 
is an area of ongoing research. Unlike the representation of the wave function in an ordinary atomic close-coupling calculation, its numerical representation in this approach gives no immediate indication of how to separate elastic, discrete inelastic and ionization channels which physically overlap on any finite volume. For single-electron targets, distorted-wave rearrangement theory can be used to develop integral expressions for computing the ionization amplitudes directly from the computed wave function. For many-electron targets, however, the problem is vastly more complicated and the use of distorted-waves alone does not Iead to stable expressions for the ionization amplitude. We have instead developed an 'asymptotic subtraction' approach in which we first compute the two-body scattering amplitudes and then use them to eliminate the contribution of discrete-channel contributions to the computed wave functions. These 'corrected' wave functions can then be used either in integral expressions for the ionization amplitudes or to compute the differential ionization flux. This procedure, while not entirely satisfactory, can be systematically improved by increasing the size of the numerical grid and does appear to offer the prospect of providing the first single differential ionization cross sections for the electron-helium system. It can also be used to compute ionization in the presence of rearrangement collisions, as one encounters in collisional ionization by positron impact.

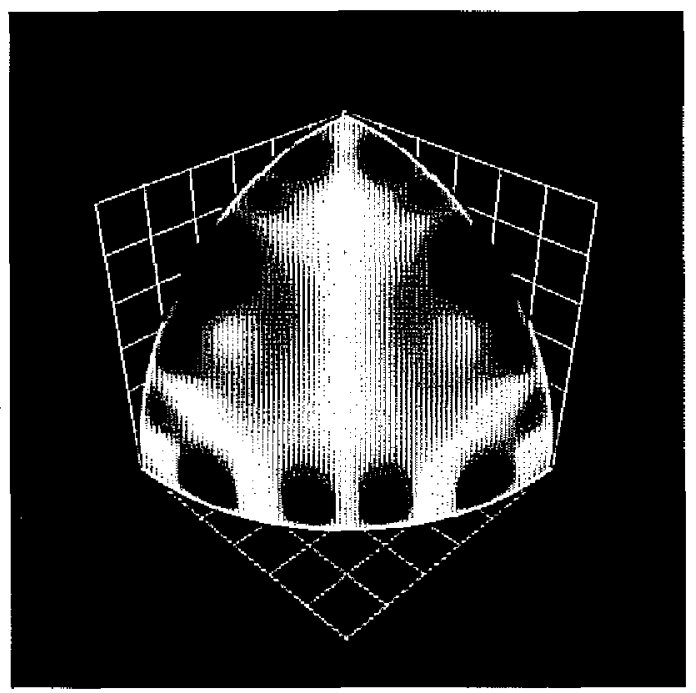

Figure 7: Wave function for a model three-dimensional simulation of electron impact on atomic helium. The concentration of density near the axes corresponds to single ionization events. The density near the center of the inage describes double ionization events which produce three free particles in the final state

\section{Publications}

C. W. McCurdy, M. Baertschy, W. A. Isaacs and T. N. Rescigno, "Reducing Collisional Breakup of a Systen of Charged Particles to Practical Computation: ElectronImpact Ionization of Hydrogen, Photonic, Electronic and Atomic Collisions," Invited Papers, Proceedings of the XXII International Conference on Photonic, Electronic and Atomic Collisions, Santa Fe, NM 200I (Rinton Press, Princeton, 2002).

C. W. McCurdy, D. A. Horner and T. N. Rescigno, "Timedependent Approach to Collisional Ionization Using Exterior Complex Scaling," Phys. Rev. A 65, 042714, (2002).

T. N. Rescigno and C. W. McCurdy, Collisional Breaktip in Coulomb Systems, Many-Particle Quantum Dynamics in Atoms and Molecules, edited by V. Shevelko and J. Ullrich (Springer-Verlag, Heidelberg), submitted for publication 2003. 



\section{Earth Sciences Division}

\section{Microbial Controls on Metals in the Environment}

Principal Investigators: Jillian Banfleld

Project No.: 02020

\section{Project Description}

This project is to conduct a molecular-level investigation of an acid mine drainage (AMD) biogeochemical system. The goal is to develop a detailed model that integrates measurements of the geochemical and biochemical characteristics of the system over time. This work requires that we monitor microbial populations in terms of their size and species makeup, as well as environmental parameters such as temperature, $\mathrm{pH}_{+}$ionic strength, metal concentrations, and redox potential. Into this framework we will incorporate genomic and gene expression data as these become available through ongoing and future collaborations. Through model-based analysis we will test hypotheses about the ecology and the form of the interconnections between geochemical characteristics and microbial activity.

Treatment of AMD solutions usually involves one of two strategies to remove metals: neutralization-oxidation, leading to oxide/oxyhydroxide precipitation and metal sorption reactions, or induction of unaerobic conditions through addition of organic compounds, leading to sulfate reduction and metal sulfide precipitation. The products of both oxidation and reduction reactions are nanocrystalline. We propose to investigate the dependence of metal sorption on nanocrystal size, the mechanisms by which metal ions such as lead and uranium are sequestered into iron oxyhydroxides, and their fate as nanocrystals grow and undergo transformations to new structures. We predict that the 1- to 3-nanometer diameter particles grow via an oriented aggregation mechanism and that the kinetics of the phase transformation are size dependent and vary with the crystal growth pathway. We will evaluate these hypotheses using sulfides synthesized via biological and non-biological pathways, hydrothermal kinetics experiments, electron- and $x$-ray-based characterization, and kinetic and structure modeling.

\section{Accomplishments}

In year 1 of this work we determined that a subset of the protists in acid mine drainage microbial communities have alphaproteobacterial endosymbionts [detected via fluorescence $i t$-situ hybridization (FISH) using speciesspecfic oligonucleotide probes]. We have demonstrated that the habitat within the protist has circum neutral $\mathrm{pH}$. This, in combination with absence of evidence for free-living cells, raises the question of whether the alphaproteobacteria is acid tolerant, and transferred only by cell division. Analysis of genes common to closely related cultivated bacterial species and mitochondria suggest a close evolutionary relationship between the alphaproteobacteria and mitochondria. The existence of intervening sequence of what appears to be functional ribonucleic acid (RNA) in variable region 1 of the alphaproteobacterial rRNA may reflect an early stage in the development of an obligate endosymbiosis. The paper reporting this first extreme acid sybiosis is in co-author review for immediate submission.

Significant effort in the past year has been dedicated to cultivation efforts by Ian Lo. We have been able to establish what appears to be pure culture of Leptospirillum (do not grow on plates, and must be isolated via solution subculturing). We have also isolated a new Thermoplasmalineage organism and shown that it grows anaerobically by coupling respiration of organic carbon to ferric iron reduction. This is the first documentation of an anaerobic pathway in the acid mine drainage system.

We have continued to work on Ferroplasma genome data and analyzed several candidate lateral gene transfer events that have shaped the metabolic activity of the AMD consortia. In particular, we have determined that there have been many separate transfers of individual genes and small gene clusters of genes between Ferroplasma and the distantly related archaeal Sulfolobus lineage. We also analyzed the history of transfers between Sulfolobus and the Thermoplasmatales linenges fairly closely related to Ferroplasma. Results indicate that different gene sets are involved. Evaluation of the functions of genes transferred indicates that certain clusters of orthologous groups (COG) (functional) groups are favored (e.g., amino acid synthesis).

We have analyzed the Ferroplasma genome for evidence of mechanisms of genome evolution. The genome contains a very large number of transposons from an anomalously large number of transposase families. We have also identified candidate prophage. Furthermore, via a new 
collaboration, we have confirmed the existence of an active bacteriophage population in our communities.

We have constructed libraries for analysis of environmental microbial community deoxyribonucleic acid (DNA) and obtained initial sequence data for the first of these (a small insert library). Mathematical analysis of the data indicates the total community metagenome size. Results indicate that the majority of each species population biomass in the community share highly related genomes, a completely new finding.

We have used coupled geochemical (Druschel) and moleculur microbiological (Baker) methods to define seven distinct communities within the AMD system that differ in microbial population, $\mathrm{pH}$, temperature, metal and sulfate concentrations. The libraries constructed in year 1 have now been fully analyzed and the results written up for publication. This work includes description of a cluster of new Thermoplasmatales lineages. In addition, a very large clone library was analyzed ( 384 clones) via high-throughput sequencing. The library was constructed for the target community for the community genomics analysis using specially-developed degenerate primers. Our results confirm the earlier claim that the communities have low species numbers. The results of this analysis, in combination with the metagenome size analysis, are in preparation for submission to Nature.

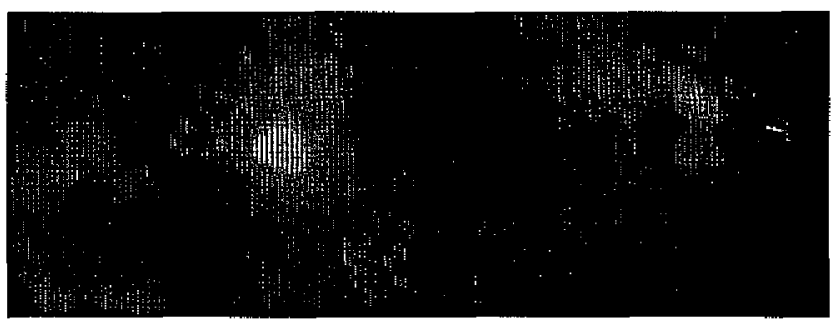

Figure 1: Epifluorescencr image of alphaproteobacterial endosymbionts within a protist in a pH 0.7 acid mine drainage microbial community. The bacterial endosymbionts are labeled with an oliognucleotide probe (lighter). DNA within the nucleus of protists is labeled with DAPI (darker).

\section{Publications}

J. Macalady and J.F. Banfield, "Molecular" Geomicrobiology: Genes and Geochemical Cycling," Frontiers: Earth and Planetary Science Letters, in press.

D.K. Newman and J.F. Banfield, "Geomicrobiology: Elucidation of the Molecular-scale Interactions that Underpin Biogeochemical Systems," Science, 296: 10711077, (2002).
B.J. Baker and J.F. Banfield, "Microbial Communities Associated with Acid Mine Drainage," FEMS Microbiology Rev, in press.

G.K. Druschel, B.B. Baker, T.H. Gihring, and J.F. Banfield, "Acid Mine Drainage Biogeochemistry at Iron Mountain, California," Geochemical Transactions, in preparation.

B. Baker, P. Hugenholtz, S. Dawson, and J.F. Banfield, "Circum Neutral pH Habitats within Extremely Acidophilic Protists Host a Rickettsiales Lineage with Intervening Sequence (IVS) in their rDNA," Applied and Environmental Microbiology, in preparation.

G. Tyson, P. Hugenholtz, W. Getz, J. Boore, and J.F. Banficld, "Microbial Communities: Many Cells, Few Genomes (tentative)," Nature, in preparation.

\section{Atmospheric Chemistry and Climate on Extrasolar Bodies: Experimental Constraints for Predictions of Habitability and Spectroscopic Signatures of Life}

Principal Investigators: Kristie Boering

Project No.: 02021

\section{Project Description}

We are investigating the production of aerosols and their optical properties in a variety of solur system atmospheres relevant to the giant planets, Saturn's moon Titan, and the early Earth and "terrestrial-like" extrasolar planets. As a first step, we are investigating photochemical haze production in both Titan-like atmospheres and terrestriallike atmospheres. For early Earth, the experiments may help answer a long-standing question: How did the early Earth maintain liquid water on its surface, despite a fainter Sun? Modeling work by James Kasting of Penn State suggests that the photolysis of $\mathrm{CH}_{4}$, produced biotically in an early Earth atmosphere composed of $\mathrm{CO}_{2}, \mathrm{~N}_{2}$, and water vapor, could produce a photochemical haze of particles as $\mathrm{CH} 4$ is photolyzed and then polymerized. Depending on the chemical composition and size of the particles, the haze could act to significantly warm (or cool) the surface. It could also provide an early uttra-violet (UV)-shield which would influence radiative transfer, the stability of organic molecules at the surface, and the photochemical lifetime of a number of other gases, and, hence, could have influenced the climate and evolution of life on Earth over the first 2 
billion years of Earth history. However, whether such an aerosol would warm or cool the surface and whether it would shield the surface from UV radiation is highly dependent on the resulting particle size distribution and index of refraction (and, hence, chemical composition) of the aerosol. Although some experimental data are available for Titan-like atmospheres, no data at all exist for terrestrial-like atmospheres. Thus, models that use arbitrary rates of particle formation are used for predictions of early Earth-like environments.

In order to address these issues, we proposed to complete and petform experiments with a unique new apparatus which enables us to measure in sitt net particle formation rates and optical properties in model gas mixtures exposed to UV radiation. Gas phase species are measured in reat time with an online mass spectrometer while particle formation rates are monitored simultaneously by optical scattering. The simultaneous evolution of both gas phase chemistry and aerosol production will provide a new double constraint for photochemical and microphysical models of planetary aerosols.

\section{Accomplishments}

In this project, we were able to expand research activities in a completely new direction - experimental "astrochemistry" -- with the promise of future significant interactions with Berkeley Lab, CalSpace/Space Sciences Lab, and faculty in the Astronomy department. Funding was used to support an exceptional second year graduate student, Mate Adamkovics, as well as equipment and supplies for our new experimental apparatus and travel to report our new results at national scientific meetings.

The design and fabrication of instrument components for a unique new apparatus in my laboratory which enables us to measure in situ net particle formation rates and optical properties in model gas mixtures exposed to UV radiation was completed during the first part of the project. Instrument optimization and performance characteristics were then studied, and experiments carried out on the production of aerosol through UV irradiation. We now have results for the irradiation of pure gas-phase $\mathrm{CH}_{4}$ at a number of different pressures and have submitted these results for publication in JGR-Planets. Gas phase species are measured in real time with an online mass spectrometer while particle formation rates are simultaneously monitored by optical scattering. The simultaneous evolution of both gas phase chemistry and aerosol production provides a new double constraint for photochemical and microphysical models of planetary aerosols. These early results already provide these constraints for the kinetics schemes in planetary atmospheric models, and we are beginning collaborations with several modeling groups on detailed analyses of these new data. We have also recently designed and implemented a new means of measuring and monitoring the particle size distribution and complex index of refraction through measurements of the angular dependence of particle light scattering through a computercontrolled rotation stage for the scattering detector. This latest addition to our experiment provides an additional capability unique to our apparatus for determining the aerosol optical properties "in situ" (i.e., while still suspended in the gas phase as opposed to measurement after deposition to a thin film which is the way this information has always been derived in the past).

Overall, this new suite of laboratory measurements is significantly enhancing, and with future experiments will continue to enhance, our understanding of the mechanism and kinetics of haze formation in planetary atmospheres and will guide a more accurate representation of these processes in models. Immediate applications, in addition to our longer-term goals of making more robust predictions of climate on the early Earth and extrasolar terrestrial-like planets, include more exacting constraints for use in the modeling of Titan's haze from previous observations of Titan's geometric albedo and thermal emission, as well as new observations expected from the Cassini orbiter and the Huygens probe in 2004 and from ground-based atmospheric image and spectroscopic data obtained with the Keck adaptive optics and other ground-based astronomical studies. Results will also be used to better predict the range of spectroscopic characteristics that may be observable in extrasolar terrestrial-like atmospheres, which, in turn, may help to refine the design requitements of space-based interferometers such as the Terrestrial Planet Finder. To continue research on these ideas with this new instrumentation, we are pursuing follow-on funding through National Aeronautics and Space Administration (NASA)"s planetary atmospheres program; the proposal is currently under review.

\section{Publications}

M.A. Adamkovics and K.A. Boering, "Photochemical Formation Rates of Organic Aerosols Through Timeresolved in situ Laboratory Measurements, ", Joumal of Geophysical Research - Planets, Decembet, 2002, submitted for publication.

M.A. Adamkovics and K.A. Boering, "Photochemical Formation Rates of Organic Aerosols Through Timeresolved in situ Laboratory Measurements," American Geophysical Union Fall 2002 Meeting, San Francisco, CA, December 9, 2002.

K.A. Boering and M.A. Adamkovics, "Hydrocarbon Hazes on Early Earth: Experimental Constraints on Photochemical Formation Rates and Optical Properties," Americarl Chemical Society Fall 2002 Meeting, Boston, MA, August $18,2002$. 
M.A. Adamkovics and K.A. Boering, "Photochemical Formation Rates and Optical Properties of Hydrocarbon Aerosol Haze Through Time-resolved, in situ, Laboratory Measurements," Second Astrobiology Science Conference, Moffett Field, CA, April 9, 2002,

\section{The Complete Genome Sequences of Hyperthermophilic Bacteria Can Tell Us About the Nature of the Early Earth's Environment}

Principal Investigators: Jeffrey Boore, Brent Mishler, and Carrine Blank

Project No.: 02022

\section{Project Description}

The purpose of this project is to generate clones in order to sequence the complete genomes of several uncultured hyperthermophilic bacteria that live in silica-depositing hot springs in Yellowstone National Park. Microorganisms will be collected in this study by incubating glass microscope slides (acting as growth surfaces) in specific near-boiling silica-depositing hot springs in Yellowstone National Park, Wyoming. The sampling of these springs will be noninvasive and non-destructive. Cells will be collected by scraping the growth surfaces with a razor blade and genomic chromosomal deoxyribonucleic acid (DNA) will be extracted from the cells. The DNA from lineages of interest will be concentrated and clone libraries will be constructed using stundard molecular biological techniques. Clones will then be submitted for sequencing at the Department of Energy/Berkeley Lab Joint Genome Institute (JGI) in Walnut Creek, California.Description

\section{Accomplishments}

Carrine Blank was a graduate student in the Department of Integrative Biology at U.C. Berkeley for spring and summer semesters of 2002. During that time, she completed phylogenetic analyses on the bacterial domain using whole genome sequences from 53 bacterial species. These sequences were obtained from public databases, aligned, and used for phylogenetic analyses using PAUP* (Phylogenetic Analyses Using Parsimony - *And Other Methods, $\mathrm{v}$ 4). A new compartmentalization method was used where monophyletic groups (groups with a single common ancestor) were identified, and smaller analyses were performed on these monophyletic groups, followed by larger analyses on multiple monophyletic groups. In the new procedure, the topology obtained by local analyses on monophyletic groups was used to constrain the relationships in that group in larger, global analyses. This new method was shown to improve the resolution of deep branching relationships in the bacterial domain.

Once a well-resolved phylogenetic tree topology for bacteria was obtained, the branching relationships among bacteria involved in mesophilic sulfate reduction, oxygenic (oxygen-producing) photosynthesis, and pyrite oxidation were compared with the geochemical record of sulfur isotope fractionation, oxidation of the atmosphere and shallow-water marine environments, and the timing of the increase in sulfate concentrations in the marine environment during the Archean and Proterozoic eras. Comparisons between the phylogenetic record and the geochemical record showed that age constraints could be assigned to the origin of these three groups of bacteria. Using whole bacterial genome sequences and the compartmentalization method for phylogenetic reconstruction, a well-resolved tree for the bacterial domain was obtained. While standard phylogenetic aralyses resulted in a moderately-well resolved tree, the compartmentalization procedure was successful at increasing the bootstrap support of all deepbranching relationships to an average value of $92 \%$. Wellresolved trees were only obtained when taxa with very long branches (i.e., the pathogenic bacteria which are evolving at elevated rates) were removed from the analyses, and when the most slowly evolving genes were used.

Comparisons of the divergence patterns of mesophilic sulfate reduction, oxygenic photosynthesis in the Cyanobacteria, and pyrite oxidation in the Proteobacteria and Low $\mathrm{G}+\mathrm{C}$ gram positive bacteria with the early Earth's geochemical record permitted the assigning of origination dates for these lineages. The inferred origin of mesophilic sulfate reduction is $\sim 2.4 \mathrm{Ga}$ ( $\mathrm{Ga}=$ billion years ago), the Cyanobacteria between 2.35 and $2.2 \mathrm{Ga}$, and the origin of pyrite oxidation between about $2.2 \mathrm{Ga}$ and $1.9 \mathrm{Ga}$. These age constraints are much later in the geologic record than have been previously assumed, however these previous assumptions were formed when a well-resolved phylogenetic tree for these organisms was lacking.

This work comprised two chapters of Dr. Blank's thesis, which was approved and submitted at the end of the 2002 summer semester.

\section{Publications}

C. Blank and B. Mishler, "Phylogeny of the Domain Bacteria: Using Compartmentalization to Better Resolve 
Ancient Divergences in the Tree of Life," in review, Molecular Phylogenetics and Evolution, August, 2002.

C. Blank, "Evolutionary Timing of Mesophilic Sulfate Reduction and 'The Great Oxidation Event': A

Phylogenomic Approach," Astrobiology, submitted for publication, October 2002.

C. Blank, B. Mishler, "Ancient Divergences in the Bacterial Tree of Life: A Total Evidence Genomic Approach", Gordon Conference on the Origin of Life, January 2002.

C. Blank, "Using the Geologic and Genomic Molecular Phylogenetic Records to Better Understand the Coevolution of Microbial Metabolisms and the Early Earth Environment," NASA Astrobiology Institute Annual Meeting, March 2002.

C. Blank, "Could Cyanobacteria Have Provided the Source of Oxidants for Banded Iron Formation?"2 2002 Annual Geologic Society of America Meeting, October, 2002.

\section{Aerobic Bioremediation of Landfills}

Principal Investigators: Terry Hazen, Curtis Oldenburg, and Sharon Borglin

Project No:: 00010

\section{Project Description}

The purpose of this research is to demonstrate and model aerobic bioremediation of municipal solid landfills as a more cost effective and environmentally sound way of managing municipal solid waste. This work is applicable not only to municipal solid waste, but also to remediation of solid waste landfills created by industry and the industrial processes of Department of Energy (DOE) and Department of Defense (DOD). The goals are to determine the critical biological and chemical parameters that control the ability of air injection and leachate recirculation in landfills to: (1) increase the biodegradation rate of the refuse mass, (2) decrease production of greenhouse gases $\left(\mathrm{CH}_{4}\right),(3)$ regulate biogenic temperature, (4) reduce metals leaching, (5) increase short-term subsidence, and (6) increase the long-term stability of the refuse mass. An increased subsidence of only $15 \%$ could translate to over $\$ 1$ billion in additional revenues for the 3500 landfills currently in operation in the United States.
Using data collected over the last year from a demonstration conducted at the Columbia County, Georgia, Landfill, and a literature evaluation, critical biological, physical, and chemical parameters have been identifted during the first year of the project. These potentially controlling parameters will be tested in the laboratory at Berkeley Lab using landfill simulation columns. The columns will be tested using various types of compaction, air injection strategies and rates, and leachate recirculation strategies and rates. During the first year, the landfill columns were designed and constructed and testing should begin shortly. The tests will be evaluated in relationship to the six goals stated above. These tests will be used to develop a numerical simulation model of aerobic bioremediation of landfills, building on the TOUGH2 code of Berkeley Lab. This approach will then be field demonstrated in the more arid environments at California municipal landfills and western DOE sites. Both the Yolo County and University of California at Davis Iandfills have been contacted.

\section{Accomplishments}

In FY02, further experimental work on the landfill bioreactors was focused on gaining more detailed data on the chemical constituents in the leachate and a more complete microbiological evaluation of both the municipal solid waste (MSW) and the leachate. Laboratory bioreactors built and filled with MSW in FY01 were run for a total of 500 days. In February 2002, the tanks were emptied and refilled with fresh refuse to replicate the results of the earlier experiments and to measure some additional parameters.

Extensive measurements were performed on the leachate coming from the landfill bioreactors, including metals, biological oxygen demand, chemical oxygen demand, organic carbon, phosphate, nitrate, ammonia, sulfides, and $\mathrm{pH}$. One of the key issues in landfill management is the control of leachate produced by the MSW. Engineering measures to prevent release of the leachate to the surrounding environment, as well as removal and treatment of leachate is expensive. The main economic and environmental advantages of aerobic and anaerobic bioreactors are that the leachate is recycled into the MSW, providing both a method of leachate treatment and disposal. The main findings from our quantitative measurements were that the anaerobic tank had elevated levels of several metals, $\mathrm{BOD}_{5}, \mathrm{TOC}, \mathrm{DOC}$, and dissolved nutrients. This indicates that the organic component of the MSW in the anaerobic tank was not as fully degraded as in the aerobic tank, and that aerobic treatment of MSW increases degradation rates and helps to reduce the environmental hazards associated with the metals and oxygen demand in the leachate. 
Further sampling of the leachate was undertaken to evaluate the microbiological community and activity in the bioreactor system. The bioreactor consists of three main components: the MSW, the gravel layer uses for drainage, and the leachate. Several assays were completed on all three components including cell counts, enzyme activity, lipid community analysis, and deoxyribonucleic acid (DNA) analysis. Key results were that the activity and cell count were higher in the anaerobic leachate than the aerobic leachate. However, activities in the gravel and in the MSW were higher in the aerobic reactors. This collaborated the findings that the leachate of the aerobic system was much cleaner, thereby not providing sufficient nutrients and food for microbial growth. Lipid extraction to determine the types of communities present in the reactors is complete, but analysis of the data is ongoing. Two undergraduate students and Professor from the University of Puerto Rico worked with us on the microbiological analysis last summer, an additional student also worked on the microbiological analysis. All three students presented posters at the end of the summer. They demonstrated that the community structure was more complex on the substrate from the aerobic reactor by DNA analysis, phospholipid fatty acid analysis and microbial activity measurements, but the opposite was true of the leachate coming from the reactors. They also showed that leachate from the Yolo County landfill was very similar in community structure to our anaerobic bioreactors.

The third main sampling effort was to continue to monitor landfill gas composition. In the replication experiment, establishment of methanogenic conditions was much more rapid due to inoculation available from the previous experiment. In the aerobic tank, oxygen respiration rates were similar to previous measurements. These data will be used to calculate degradation rates, carbon mass balances, and to demonstrate changes in the condition of the tanks over time.

We continued development and testing of T2LBM, the Landfill Bioreactor Model for TOUGH2. The main efforts were aimed at completing the users guide, and applying the model to the laboratory experiments. Application of T2LBM to the laboratory experiments showed that the model could simulate the depletion of oxygen and transition to anaerobic conditions during a shut-in test (where the flow of air in to the MSW is stopped for a period or a day or two). These radjal two-dimensional $(r-Z)$ results also include leachate flow downward through the MSW, gas flow upwards, and heating due to biodegradation.

Contact and collaborations were fostered with Yolo County Central Landfill in Woodland, Culifornia. Several visits were made to the landfill site to collect samples and to share information. This collaboration will result in future publications and possible collaboration on applications for funding. An EMSP proposal will be submitted for FY 2004. Collaboration with Technical Transfer has been established to develop an intellectual property license for smart storage technologies and for the T2LBM module for TOUGH2. The result of this collaboration will be the development of an expanded web site to advertise our capabilities to municipalities and government agencies who would be interesting in funding further research in the area of aerobic remediation of landfilled waste.

We also proposed and were approved to organize a special session in the Biogeosciences section at the American Geophysical Union (AGU) Full 2002 conference in San Francisco. This session will bring together an international group of scientists in the area of waste storage and biotreatment of landfilled waste. This session will help foster communication and collaboration between our research group and other scientists.

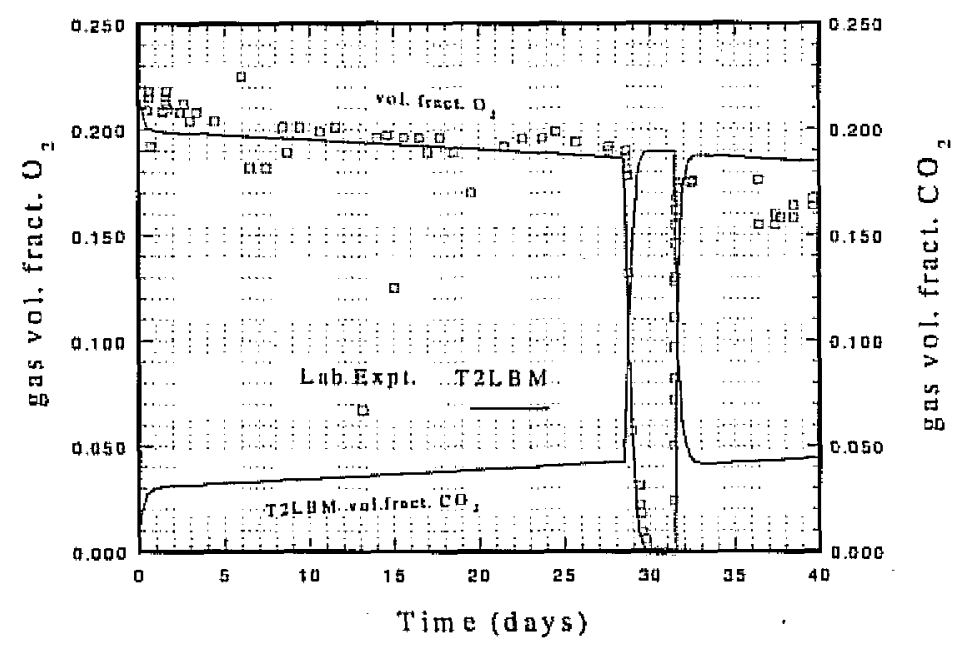

Figure 2: Volume fractions of $\mathrm{O}_{2}$ from the laboratory experiment and T2LBM and voltume fraction $\mathrm{CO}_{2}$ from T2LBM.

\section{Publications}

C.M. Oldenburg, T2LBM Version 1.0: Landfill Bioreactor Model for TOUGH2, LBNL-47961, November 2001.

C.M. Oldenburg, S.E. Borglin, and T.C. Hazen, ${ }^{+}$Process Modeling of Flow, Transport, and Biodegradation in Landfill Bioreactors," Fall Meeting of the American Geophysical Union, LBNL-48904, September 2001,

S.E. Borglin, T.C. Hazen, C.M. Oldenburg, and P.T. Zawislanski., "Mesoscale Laboratory Models of the Biodegradation of Municipal Landfill Materials," Fall Meeting of the American Geophysical Union, LBNL48884, September 2001. 
M. Greenberg, T. Hazen, S. Borglin, and C. Oldenburg, "Factors Controlling Metals Concentration in the Leachate from Aerobic and Anaerobic Laboroatry Landfill Bioreactors," Fall Meeting of the American Geophysical Union, LBNL-48928 Abs, September 2001.

\section{S. Borglin, T.C. Hazen, C. Oldenburg, and P.T.} Zawislanski, "Mesoscale Laboratory Experiments of Aerobic and Anaerobic Biotreatment of Municipal Solid Waste," in preparation.

S. Borglin, T.C. Hazen, C. Oldenburg, "Stabilization of Stored and Landfilled Waste using Aerobic and Anaerobic Biotreatment Technology," The Second Intercontinental Landfill Research Symposium, October 2002.

S. Borglin, T.C. Hazen, C. Oldenburg, "Smirt Storage: Stabilization of Stored and Landfilled Waste using Aerobic and Anaerobic Biotreatment Technology," 2002 Partners in Environmental Technology Symposium \& Workshop, December 3, 2002.

T.C. Hazen, "Aerated Landfills: Changing the Dry Tomb Paradigm," First International Conference on Environmental Recovery of Yugoslavia, September 2001.

C.M. Oldenburg, S.E. Borglin, and T.C. Hazen., "Simulations of Flow, Transport, and Biodegradation in Landfills," Fall Meeting of the American Geophysical Union, in preparation.

S.E. Borglin, T.C. Hazen, and C.M. Oldenburg, "Comparison of Leachate Quality from Aerobic and Annerobic Municipal Solid Waste Biorenctors," Fall Meeting of the American Geophysical Union, in preparation.

T.C. Hazen, S.E. Borglin, and C.M. Oldenburg, "Aerobic Landfill Bioremediation," In Situ and On Site Bioremediation: The Sixth International Symposium June 2003, in preparation.

S.E. Borglin, T.C. Hazen, "Microbial Community Structure in Aerobic Landfill Bioreactors," in preparation.

S.E. Borglin, T.C. Hazen, A.A. White, "Characterization of Metals Leaching from Aerobic and Anaerobic Landfill Bioreactors," in preparation.

A.A. White, S.E. Borglin, and T.C. Hazen, "Mesoscale Aerobic and Anaerobic Lindfill Bioreactors," submitted to Journal of Undergraduate Research, December 2002.

A.A. White, S.E. Borglin, and T.C. Hazen, "Mesoscale Aerobic and Anterobic Landfill Bioreactors," American Association of the Advancement of Science Meeting, in preparation.

\section{Coupling of Seismologic and Hydrologic Processes}

Principal Investigators: Michael Manga

Project No.: 02023

\section{Project Description}

We will document the coupling of earthquakes and hydrologic processes in natural geologic systems, both in space and in time. Understanding this coupling will allow us to predict the conditions under which hydrologic changes (for example, those caused by fluid injection, fluid extraction, and/or changes in subsurface temperature) may induce seismicity. Understanding natural systems provides a baseline for interpreting observations in engineered systems. We will search for statistically significant correlations in space and time between earthquakes and hydrologic processes. Two specific problems will be studied: (1) earthquake triggering by natural groundwater recharge and (2) changes in streamflow following earthquakes. Observed behaviors will be interpreted with poroelastic flow models.

\section{Accomplishments}

Earthquakes at some volcanoes, such as Mt. Hood in Oregon and Mt. Lassen in California, are located off-center from the axis of the volcano. We find statistically significant correlations (see Figure 3) between groundwater recharge and seismicity at $\mathrm{Mt}$. Food, Oregon. The time lag of about 150 days between recharge and seisnicity allows us to determine the regional hydraulic diffusivity $\left(10^{0} \mathrm{~m}^{2} / \mathrm{s}\right)$ and other hydrogeologic parameters (permeability of $10^{-13}$ $\mathrm{m}^{2}$, vertical matrix compressibility of $10^{-10} \mathrm{~m}^{2} / \mathrm{N}$ ). These values are comparable with those we determine independently by using coupled heat and groundwater flow models to interpret bore hole temperature data at Mt. Hood. We thus conclude that hydroseismicity occurs in this region and is caused by natural groundwater recharge. This result also suggests that faults in this area are near criticallystressed. Discerning whether hydroseismicity can be induced by pore fluid pressure changes as small as the 0.1 to 0.2 megapascal estimated during groundwater recharge, has implications for seismic risk evaluations related to reservoir impoundment, subsurface injection of waste fluids, carbon sequestration, geothermal energy exploration, and estimates of the state of stress in the crust. In addition, by employing this approach, hydrogeologic properties such as permeability and specific storage can be estimated on a large spatial scale. 
We have also investigated the effect of earthquakes on streamflow in order to understand the effect of seismic waves on pore pressures in aquifers. We analyzed the response of most of the gauged streams in California to historical earthquakes. We focused the analysis on Sespe Creek in southern California because its discharge changed after several earthquakes. The magnitude and occurrence of changes in sireamflow are best explained by consolidation of alluvial deposits. We also show that changes in streanflow cannot be caused by changes in uquifer permeability.

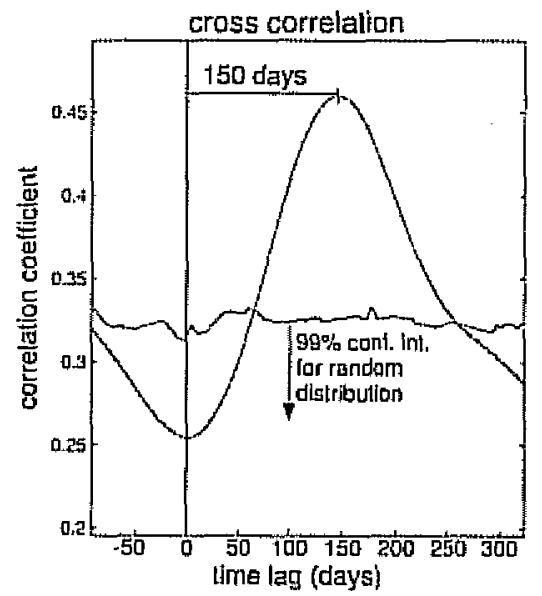

Figure 3: Normalized unbiased cross correlation coefficients as a finction of time lag for strean discharge in Salmon River, Oregon, correlated with total daily seismic moment at Mt. Hood, Oregon, for the 10-year period from 10-01-1986 through 09-30-1996. Stream discharge serves as a proxy for groundwater recharge. Also shown are the upper bounds of the $99 \%$-confidence intervals for random earthquake distribution

\section{Publications}

M.O. Saar and M. Manga, "Seismicity Induced by Groundwater Recharge at Mt. Hood, Oregon, and its Implications For Hydrogeologic Parameters," Eos Trans. $A G U, 83(47)$, Fall Meet. Suppl., Abstract G51A-0944, 2002 , accepted for publication.

M. Boone, M. Manga, and E.E. Brodsky, "Origin of Postseismic Streamflow Changes Inferred from Baseflow Recession and Magnitude-Distance Relations," Eos Trans. $A G U, 83(47)$, Fall Meet. Suppl., Abstract G51A-0946, 2002, accepted for publication.

M. Manga, E.E. Brodsky, and M. Boone, "Response of Streamflow to Multiple Earthquakes and Implications for the Origin of Postseismic Discharge Changes," Geophysical Research Letters, 2002, submitted for publication. Seismo.berkeley.edw/manga/paper60.pdf

\section{Development of Monitoring Strategies for Carbon Sequestration Verification Using Coupled Subsurface and Subaerial Simulation}

\author{
Principal Investigators: Curtis Oldenburg \\ Project No.: 02024
}

\section{Project Description}

The accumulation of vast quantities of $\mathrm{CO}_{2}$ in geologic sequestration sites entails health and environmental risk from potential leakage of $\mathrm{CO}_{2}$ to the near-surface environment. The objective of this project is to use detailed coupled simulations of subsurface and subaerial migration of $\mathrm{CO}_{2}$ from geologic sequestration sites to predict subaerial and indoor $\mathrm{CO}_{2}$ concentration distributions under a variety of geologic and meteorological conditions. These scenarios will provide the basis for determining aboveground and near-surface instrumentation needs for effectively monitoring carbon sequestration to ensure public safety,

Three areas of work will be carried out in this project: (I) development, verification, and application of coupled subsurface-subaerial flow simulation capabilities using TOUGH2, (2) assessment of state-of-the-art instrumentation and monitoring strategies for $\mathrm{CO}_{2}$ flow over variable topography and in buildings, and (3) analysis and interpretation of simulation results for design and specification of instrumentation and monitoring needs for detecting leaks under various scenarios.

\section{Accomplishments}

While the main single effort during the first year of this project was development of the simulation capability for modeling $\mathrm{CO}_{2}$ flow and transport, we worked in a variety of areas in support of the project. Specifically, in early FY02, we focused the project on the subsurface-subaerial coupling of $\mathrm{CO}_{2}$ seepage and atmospheric dispersion because this is where surface instrumentation is likely to be used. By subaerial region, we refer to the lowest part of the atmospheric surface layer just above the ground surface, where a dense gas can be expected to persist because wind velocity is small. We further refined the language of the project so that we refer to seepage as meaning $\mathrm{CO}_{2}$ crossing a boundary between porous media and ambient air, for 
example through the ground surface boundary or through a cracked concrete basement floor. In contrast to seepage, leakage is simply the migration of $\mathrm{CO}_{2}$ away from the intended sequestration target formation entirely within the subsurface.

Literature searches were carried out in the areas of $\mathrm{CO}_{2}$ monitoring, industrial dense gas dispersion, large natural $\mathrm{CO}_{2}$ emissions, and atmospheric transport modeling. We found instrumentation for environmental $\mathrm{CO}_{2}$ monitoring falls into two broad categories: (1) Infra Red Gas Analyzers (IRGA); and (2) Light Detection and Rangefinding (LIDAR).

The new gas simulation capability that we are developing handles the flow and transport of water vapor, $\mathrm{CO}_{1}, \mathrm{a}$ passive gas tracer, and air. This simulation capability is being developed within the TOUGH2 framework and models flow of $\mathrm{CO}_{2}$ upward through complex geologic structures in the saturated and vadose zones, and into the subuerial environment where topographic and meteorological conditions will control mixing and gas migration. The approach we are taking for the surface layer combines a standard logarithmic velocity profile prescribed at the lateral boundaries with active density-coupling within the calculation domain. Testing and verification of the new capabilities are underway.

Speciftc findings to date include the following: (1) Applications of published correlations of dense gas dispersion to carbon sequestration seepage scenarios suggest that (i) $\mathrm{CO}_{2}$ dispersion in the surface layer is an active (i.e., density-dependent) as opposed to passive mixing process in calm conditions even for very small fluxes (see Figure 4) and (ii) that utmospheric dispersion will disperse seeping $\mathrm{CO}_{2}$ after $10-100 \mathrm{~m}$ of downwind travel; (2) Correlation-based predictions need to be reconciled with numerous natural examples of $\mathrm{CO}_{2}$ emissions that resulted in human and animal deaths after much longer travel distances, suggesting the potential importance of calm conditions and topographic effects; (3) Our own simulations of leakage and seepage showed that seepage fluxes of $\mathrm{CO}_{2}$ tend to be small for expected carbon sequestration leakage scenarios, while the corresponding shallow soil-gas $\mathrm{CO}_{2}$ concentrations are relatively high. These findings will be combined with additional simulation-based predictions and knowledge of instrumentation sensitivities to develop effective sequestration monitoring and verification strategies.

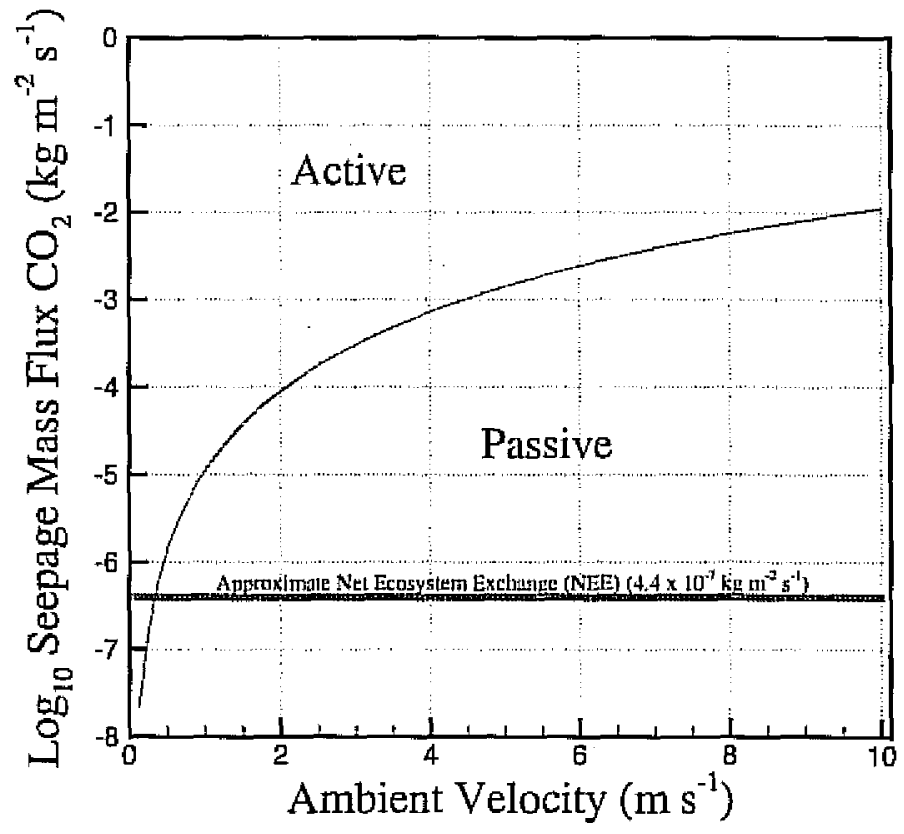

Figure 4: Criterion for active (density-dependent) vs. passive (no density dependence) flow as a function of $\mathrm{CO}_{2}$ seepage mass flux and ambient wind velocity. 


\section{Applying a Coupled Climate-Land Surface Regional Model to Deduce Trends in Soil Moisture from Air Temperature Data}

Principal Investigators: William Riley, Margaret

Torn, Marc Fischer, John Harte, and Helen He

Project No.: 02025

\section{Project Description}

The goal of this project is to develop a modeling framework to evaluate coupling and feedbacks between the land surface and atmosphere at regional to continental scales. To this end we have developed, from extant models (LSM1 and MM5), a fully coupled land-surface and atmosphere regional-scale model running at Berkeley Lab National Energy Research Scientific Computing (NERSC) Center. The coupled model allows for mechanistic treatment of the interactions between the surface energy balance and carbon exchanges via photosynthesis and predicts the net ecosystem exchange of $\mathrm{CO}_{2}$. Understanding and being able to accurately simulate energy and mass exchanges between the land surface and atmosphere is critical to our ability to predict the longterm impacts of anthropogenic activity, including continued fossil fuel combustion, land use, and watercycle changes. The development of this tool is also intended to assist other researchers who are interested in coupled interactions between atmospheric processes and carbon, energy, and water cycles.

With the coupled model we are addressing several questions: (1) Is there a discernable relationship in amplitude or phase between the diurnal temperature range and soil moisture? (2) How will large-scale human landuse, such as harvesting and fallow fields, impact regional atmospheric processes and near-surface air temperatures, soil moisture, and soil temperature? and (3) How will changes in the direct and diffuse fraction of shortwave radiation at the surface impact surface energy, water, and $\mathrm{CO}_{2}$ exchanges?

\section{Accomplishments}

We have successfully coupled the models and the code is now available and running on NERSC Center/Berkeley Lab. The parallel version results match bit-to-bit with sequential run results and the code runs $\rightarrow 38$ times faster with 64 CPUs than with a single CPU. We have tested the coupled model in two ways. First, we compared simulations to precipitation maps from several periods to test continental-scale predictions. Second, we used data from the three-year FIFE experiment to test the finer resolution results. This dataset contains short- and longwave radiation mensurements, air temperature, latent and sensible heat fluxes, and soil moisture and temperatures average over $225 \mathrm{~km}^{2}$ in Kansas. Figure 5 shows measured and predicted surface energy fluxes from this test for June 1988. Near-surface air temperature was also accurately predicted (not shown). Overall, the coupled model simulates energy exchange, precipitation, nearsurface air temperatures, and soil moisture and temperatures at least as well as the current land-surface model, and in addition provides consistent estimates of ecosystem $\mathrm{CO}_{2}$ exchange with the atmosphere.

We have performed simulations using a two-nest approach (100 km covering contiguous U.S. and $10 \mathrm{~km}$ centered over Oklahoma) to investigate the relationship between soil moisture and diurnal temperature range (DTR) and the phase and amplitude of diurnal variations of relevant quantities (e.g., air and soil temperatures, and heat fluxes). We compared nominal and dry (80\% of nominal soil moisture) simulations over months with little cloud cover and precipitation in order to isolate the impact of soil moisture. At the regional scale $(-300 \times 300 \mathrm{~km})$ no obvious patterns emerged. However, at the $10 \mathrm{~km}$ scale significant differences between treatments exist. In particular, for a month without rain, the drier soils resulted in significantly lower latent heat fluxes and an increase in the amplitude of the $2 \mathrm{~m}$ air temperature of $-0,4^{\circ} \mathrm{C}$. Differences between nominal and dry soil conditions in diurnal temperature phase were insignificant. We ure currently analyzing these patterns to determine whether these results are consistent across the landscape.

Land use can have significant impacts on regional climate. In northern Oklahoma, agriculture is the dominant land use, and wheat the predominant crop in winter through spring. During June of each year a significant fraction of the land surface is harvested and left fallow until the following planting. We are investigating the impact of this land-use change on regional-scale air temperatures, soil moisture, and precipitation. We have gathered Oklahoma land-use information (i.e., crop type and planting and harvest dates) at the county level and have incorporated this information into the coupled LSM1/MM5 model. Results from these simulations indicate that the effects of the June harvest on $2 \mathrm{~m}$ air and soil temperatures were easily detectable and followed a geographic pattern corresponding to land use and vegetation type. 
Vegetation canopies use diffuse solar radiation more efficiently than direct radiation. Anthropogenic activities such as combustion and land use and natural events such as volcanoes can impact global distributions of atmospheric aerosols, and thereby the distribution of diffuse and direct radiation. The differential response of vegetation to diffuse and direct radiation, results in substantially different energy and $\mathrm{CO}_{2}$ exchanges with the atmosphere. We have investigated the impact of this effect on the contiguous U.S. by simulating an increase in diffuse radiation consistent with increases seen after the Mt. Pinatubo eruption, while keeping the total solar radiation incident at the surface constant. Averaging the results across the contiguous U.S. shows that carbon uptake, under the higher levels of diffuse radiation over one summer month, increased by $\sim 7 \mathrm{~g} \mathrm{~m}^{-2}$, or $-25 \%$. This result is consistent for two different model years and with observations at several forest sites in the U.S. where continuous measurements of direct and diffuse radiation and net ecosystem exchange of $\mathrm{CO}_{2}$ were made in the years following the eruption.

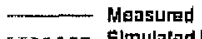

n....... Slmulated LSM1.
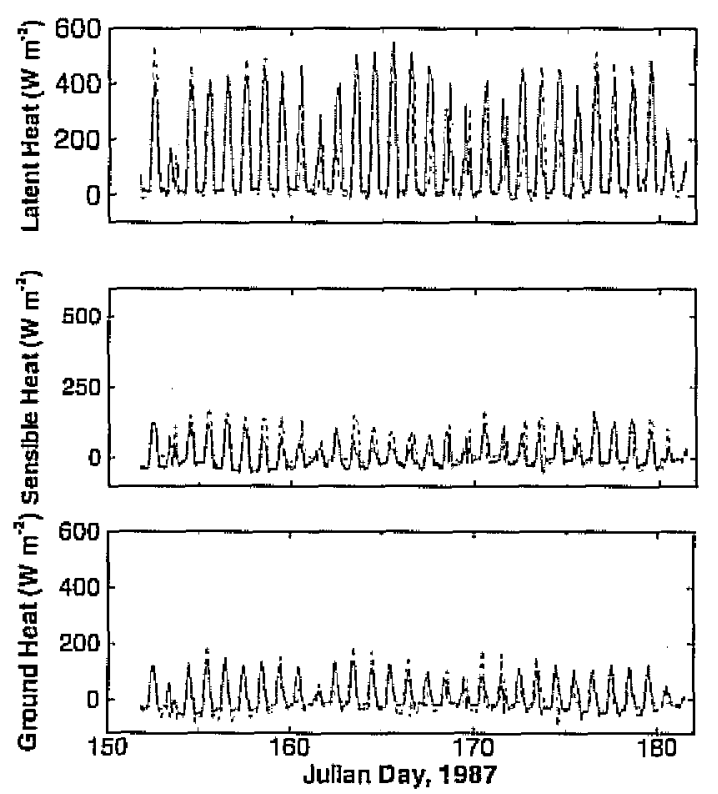

Figure 5: Simulated and measured latent, sensible, and grotund heat flutes for June 1987. Measutrements's tire from the FIFE experiment, with averages reported over a 225 $\mathrm{km}^{2}$ area in Kansas

\section{Reactivity of Nanoparticles in Natural Environments}

Principal Investigators: Glenn Waychunas, Jillian

Banfield, Hoi-Ying Holman, and Paul Alivisatos

Project No.: 02026

\section{Project Description}

This project is to investigate how nanoscale weathering and microbial biomineralization products impact the geachemistry of the environment. It will seed investigations into how new biological and geochemical processes combine to generate nanometer-scale particles, and the ways in which the reactivity and fate of these particles are size dependent. New knowledge will be applied to development of strategies for remediation of environments contaminated with metals sequestered within, or adsorbed onto, nanophase minerals. Eventually, these efforts are also expected to yield novel approaches for the creation of new impurity-doped and composite biomaterials for other technologies.

Preliminary results from ongoing small-scale research activities at Berkeley Lab and University of California, Berkeley, have revealed unexpected behavior associated with nanophase materials, suggesting that a focused research effort dealing with nanoscale particles in complex natural systems can yield important insights into environmental processes. We will investigate the pathways for nanomineral particle formation, growth, and assembly in the presence of naturally organic compounds by several complementary and mutually supportive efforts. Banfield's group will cultivate microorganisms that induce mineral precipitation due to their utilization of inorganic species as electron acceptors (Fe3+U6t, SO42-) or electron donors (Fe2+, S-), and characterize the products via transmission electron microscopy (TEM) and $\mathrm{x}$-ray diffraction (XRD). Holman's group will investigate the impact of organic species on the size distribution and growth morphology of nanomineral particles, concentrating on the specifics of organic binding at surfaces. Waychunas' group will characterize nanomineral size distributions and surface/bulk structure, and conduct real-time studies of growth and aggregation kinetics using small angle $\mathrm{x}$-ray scattering (SAXS) and $\mathrm{x}$-ray absorption spectroscopy (XAS). Alivisatos' group will investigate particle morphology and phase control via manipulation of size, growth pathway and solution chemistry. Together, these approaches will ensure new insights into the fundamental physical, chemical, and biological processes 
that control renctions at the surfaces of essentially ubiquitous nanosized particles in natural environments.

\section{Accomplishments}

\section{ZnS Nanoparticles}

The thermodynamics of small particles differ from the

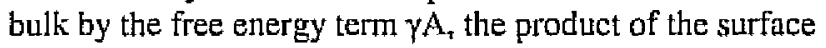
or interfacial free energy and the surface or interfacial area. As the surfaces of different crystal phases do not possess equal interfacial energies, a reversal in phase stability may occur with decreasing particle size, as observed in nanocrystalline alumina. We have determined that, for a fixed particle size, directly modifying yby interfacial water binding can cause a profound structural modification in nanocrystalline ZnS. Water binding to the nanoparticle surface reduces internal disorder and stabilizes a structure that is close to that observed in bulk $\mathrm{ZnS}$. This demonstrates the feasibility of room temperature surface-driven phase transformations in nanomaterials used for devices, sensors and novel materials. The dependence of structure on the surroundings has broad implications for the state of matter in dust and in the atmospheres of planets and in other parts of the universe where nanoparticles occur.

As a particle shrinks, the structure becomes increasingly dominated by its surface. Surface reconstructions minimize surface energy and may propagate throughout nanoparticles. Because the extent of structural distortion depends on the surface energy, the structural state should vary with the particle's environment. The surface tension of a nanoparticle varies with solution $\mathrm{pH}$. Other environmental factors that could modify the surface tension, and thus the structure, include ligand binding (1), non-specific interactions with molecules in solution, and aggregation. We have determined that the aggregation state can profoundly influence particle structure.

Our results show that the structure of $3 \mathrm{~nm} \mathrm{ZnS}$ nanoparticles differs from that of bulk $\mathrm{ZnS}$ and varies with particle aggregation state. Dispersed particles have a more disordered internal structure than aggregated particles of the same size. A reversible phase transformation between these structures can be induced by changing the aggregation state. Because the transformation occurs at room temperature, we infer that the atctivation energy is small and that nanoparticles are not trapped in a metastable state. These results reveal that the structures, and thus properties and reactivity of small particles are responsive to the particle's environment.

\section{Biogenic Nanoparticles}

We have conducted experiments to explore the underlying molecular mechanisms that control the structure and stability of biogenic $\mathrm{Cr}$ mineral phases. We selected Arthrobacter axydans from the Department of Energy's (DOE) Idaho National Engineering and Environmental Laboratory site as our model organism because it is probably one of the most Cr-resistant bacteria in vadose zones. Scanning electron microscope (SEM), Transmission Electron Microscope (TEM), and Synchrotron Radiation - Fourier Transform Infrared (SRFTIR) spectromicroscopy were used to observe the formation and speciation of $\mathrm{Cr}$ mineral phases on surfaces of A. oxydans and on the associated biomolecules.

Our results show that:

- In the presence of $\mathrm{Cr}(\mathrm{VI})$-compounds, $A$. oxydans produces $S$-layer like proteins (crystalline) that protrude outward $(-20-80 \mathrm{~nm})$ and form a composite 3-D structure (see Figure 6 and caption below).

- This S-layer protein is coated with $\mathrm{Cr}(\mathrm{V})$ compounds within a week of the first exposure, and then is completely transformed to Cr(III)compounds within one month.

- Very often the $\mathrm{Cr}(\mathrm{III})$-coated S-layers detach from $A$. oxydans and exist in the form of "laminated" $\mathrm{Cr}$ (III)-protein complexes with sizes from nanometer to micron scales. Their structure, morphology, and reactivity can have significant impact on the fate and transport of fluids and contaminants, the development and success of any remediation technologies, and our ability to predict natural attenuation for long-term $\mathrm{DOE}$ site stewardship.

\section{Nanomineral Growth and Aggregation}

We have begun an examination of the growth and aggregation behavior of the common nanomineral phase goethite $(\alpha-\mathrm{FeOOH})$ produced abiotically. Goethite is a ubiquitous in soils and sediments, always occurs in clusters of sub-micron to few micron crystallites, appears to be inherently nanoporous or nanodefective (not well characterized), and is a powerful sorbing agent for both metal contaminants and plant nutrients in the environment. The study is being done both on laboratory batehes prepared for TEM, X-Ray Diffraction (XRD) and laserscattering particle-size distribution characterization, and analogous material created in real time syntheses with continuous-flow and stopped flow reactors for in-situ small-angle $x$-ray scattering (SAXS) and wide-angle $x$-ray scattering (WAXS) studies at the Advanced Photon Source (APS) at ANL.

We are pleased to be collaborating with the scientific team at the ChemMatCARS sector at the APS where their insertion device beamline has a state-of-the-art dedicated SAXS/WAXS system that has just been commissioned. 
We will be one of the first users of this facility and will be working with the team to optimize its operation.

Coupled work on goethite nanoparticle sorption properties, specifically to understand the change in somption characteristics and the electrical double layer structure at the mineral-water interface as a function of nanoparticle size are also being undertaken at Stanford Synchrotron Radiation Laboratory (SSRL) and the APS.

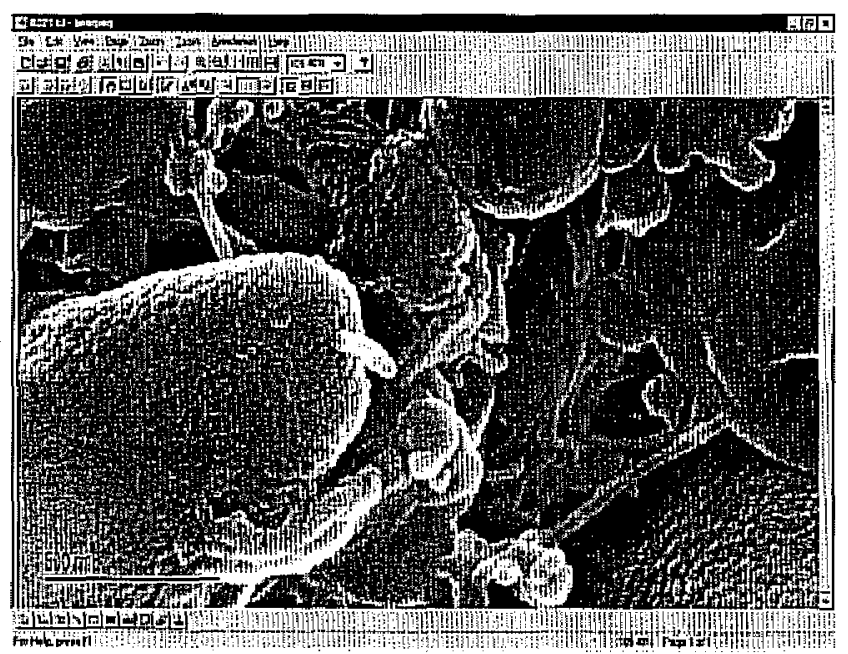

Figure 6: A SEM image of S-layer like protein crystallines produced by $\mathrm{Cr}(\mathrm{VI})$ - resistant Arthrobacter oxydans after the bacteria are exposed to $1000 \mathrm{ppm}$ cliromate. A. oxydans were isolated from the Idaho National Engineering and Environmental (INEEL) site.

\section{Publications}

H. Zhang, B. Gilbert, F. Huang, and J.F. Banfield, "Water-driven Structural Transformation in Nanoparticles," Nature, December 2002, submitted for publication.

F. Huang, H. Zhang, B. Gilbert, and J.F. Banfield, "Reversible Phase Transformations Controlled by Aggregation-disaggregation," Science, December 2002, submitted for publication.
C. Kim, J. Banfield, and G. Waychunas, "Characterization and Reactivity of Nanoparticulate Goethite," ACS National Meeting, New Orleans, 2003.

B. Gilbert, H. Zhang, F. Huang, and J.F. Banfield, "Synchrotron Analysis of ZnS Nanoparticle Structure and Surface-driven Structural Transitions," ACS National Meeting, New Orleans, 2003.

H. Zhang, F. Huang, B. Gilbert, and J.F. Bantield, "Phase Stability of Nanocrystalline ZnS by Thermodynamic Analysis and Experimental Study, "ACS National Meeting, New Orleans, 2003.

F. Huang, H. Zhang, B. Gilbert, and J.F. Banfield, "Surface State Controlled Nanocrystalline ZnS Structure Transformation," ACS National Meeting, New Orleans, 2003.

B. Gilbert, H. Zhang, F. Huang, M. Finnegan, G. Waychunas, and J.F. Banfield, "Special Phase Transformation and Crystal Growth Pathways Observed in Nanoparticles," ACS National Meeting, New Orleans, 2003.

B. Gilbert, H. Zhang, F. Huang, and J.F. Banfield, "Ligand Effects on the Structure of Nanoparticles," University of Wisconsin Synchrotron Radiation Center User's Meeting, October 25, 2002.

J.F. Banfield and $\mathrm{H}$. Zhang, "Nanocrystals in the Environment In: Nanocrystals and The Environment," Reviews in Mineralogy and Geochemistry, J.F. Banfield and A. Navrotsky, Eds. Vol. 44, 1-58., 2001.

G. Waychunas, "Structure, Aggregation and Characterization of Nanoparticles. In: Nanocrystals and The Environment," Reviews in Mineralogy and Geochemistry, J.F. Banfield and A. Navrotsky, Eds. Vol. 44, 105-166, 2001.

G. Waychunas, "Sulfate Binding in Nanocrystalline Fe Oxyhydroxides," invited paper, PNNL/EMSI Geochemistry Symposium, Richland, WA, 2002.

G. Waychunas, "How Do Bulk Crystal Surfaces Differ from Nanocrystal Surfaces?" NSF-NRC NanoGeoScience workshop presentation, LBNL, 2002, Associated report on NSF website. 



\section{Engineering Division}

\section{Fast Lipoprotein Identification Process}

\author{
Principal Investigators: Henry Benner and Ronald \\ Krauss
}

Project No.: 01040

\section{Project Description}

The measurement of lipoprotein (LP) particles in human blood is used to assess the risk of coronary heart disease (CHD). Risk is related to the size distribution and cholesterol content of lipoprotein particles. With appropriate analytical methodology, LP is classified by particle size into several well-known categories such as high density lipoprotein (HDL) and low density lipoprotein (LDL) along with additional less well-known classes and subclasses. This project evaluates a gas phase particle mobility technique for measuring the size distributions of LP for the purpose of designing a faster, more accurate and cheaper analytical method for LP. Gel electrophoresis is the most commonly used technique for revealing LP classes but it is slow and requires a welltrained operator and data interpreter. We have determined that gas phase particle mobility measurements can be used to display LP size distributions in about 3 min compared to a day or two for gel separations. Detecting mobility classified LP is based on nucleated condensation and allows LP concentrations to be determined accurately, thus circumventing the chromogenic problems encountered when lipid or protein stains are used to measure LP in gels. We have demonstrated that high risk patients can be distinguished from low risk patients on the basis of LP spectra measured with this technique.

\section{Accomplishments}

Lipoprotein particle (LP) size distributions are used to assess the risk of coronary heart disease (CHD) in humans. Classically, lipoprotein particle size distributions have been revealed by gel electrophoresis. A shift of only a few nanometers in modal diameter of low density lipoprotein particles is adequate to assign a patient to a high or low risk category. The gel technology requires several days to run and if this diagnostic test is to become available for wide spread screening of CHD risk a faster analytical methodology is needed.

We have introduced the use of gas phase particle mobility measurements to obtain LP particle size distributions. We determined that a number of significant benefits derive from mobility analysis of $L P$ compared to gel electrophoresis. To begin, mobility measurements performed at a resolution adequate to determine CHD risk from LDL size distributions can be obtained in about 3 min., which greatly increases the potential for high through put screening compared to a test that relies on gel electrophoresis. Additionally, the mobility measurement produces an output in digital form and circumvents the requirement for 'reading" a gel.

Mobility mensurements are based on aerodynamic and electrical properties of the LP particles and not on their chemical properties. Particle size distributions obtained with gel separations are influenced by the choice of stain used to reveal the distribution. Protein specific or lipid specific stains reveal the location of LP particles after a gel separation but because LP particles do not have a constant composition across all sizes, the intensity pattern of $L P$ in a stained gel is influenced by particle composition. The staining problem introduces a shift in apparent particle size and further complicates the accurate quantification of LP particles. Stains are not used in mobility measurements and therefore the staining bias is eliminated with mobility measurements, allowing more accurate measurement of particle size.

In the methodology we developed, LP particles pass thorough a mobility tube operated at atmospheric pressure, which classifies the particles according to cross sectional area, and then enter a detector that counts individual particles. The detection scheme is efficient, responds to individual particles, and is quantitative because it is based on obtaining a particle count. As a result of the design characteristics of the system, particle size spectra can be displayed as frequency (number of particles in a size bin vs. diameter) or weight (mass of particles in a size bin vs. diameter) spectra.

Another benefit derived from the use of gas phase mobility to measure LP particle size distributions is that particles are less likely to be distorted in shape as they travel through a gas mobility tube. The forces experienced by a particle in a mobility tube are small and allow the particles to maintain their spherical shape. Consequently mobility measurements produce a more accurate measure 
of particle size than gel electrophoresis where the shape of soft particles changes as they migrate tortuously through a gel matrix and as a result migration distance in a gel is less accurately assigned to particle size. Lipoprotein particle standards are available for calibrating gels but such standards are secondary standards and are only useful for assigning a relative particle diameter based on a relative migration distance and should be used cautiously when reporting absolute particle diameter.

The range of particle size addressable with mobility measurements spans several types of gels. High density lipoprotein (HDL) and LDL particle size analyses are routinely obtained with two separate types of gels. In other words, HDL and LDL size distributions can not be obtained from the same gel separation and because it is clinically valuable to measure the distribution of both types of particles, two gel runs are frequently set up to obtain the appropriate results. Furthermore, very low density lipoprotein (VILDL) particles and chylomicrons, two more types of lipoproteins, are difficult to measure with gel electrophoresis. The particle mobility technique we established is also able to reveal the distributions of these particles. The clinical value of VLDL and chylomicron size measurements has not yet been estublished in terms of CHD risk assessment but our methodology opens the possibility for such studies to be performed and may lead to further predictive capability.

We have analyzed a large number of plasma samples and compared classical methods for LP analysis with mobility measurements. We have observed that LP particle sizes obtained with gel electrophoresis correlate linearly with mobility measurements. The relationship between the two measurements is mobility diameter $=0.86$ (gel diameter). Mobility measurements are approximately linear with respect to particle concentration spanning more than two orders of magnitude. We have demonstrated conclusively that mobility measurements can be used to assess CHD. An example is presented in the attached figure.

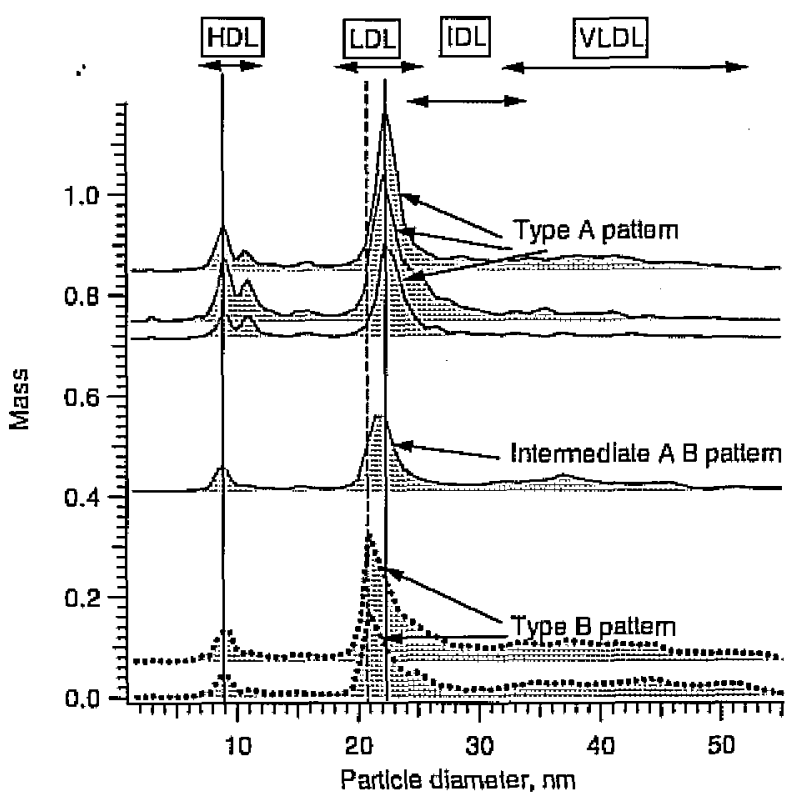

Figure 1: This figure was constructed with data obtained from a number of patients participating in a cholesterol study. Classical analysis techniques were used to identify five patients whose lipoprotein profiles distingtished them as having low, intermediate and high risk for coronary heart disease. Plasma from these five patients was then analyzed using gas phase mobility to measure their lipoprotein particles. The mobility size distributions (displayed) were in agreentent with classical measurements and led to the same rish assessment. Type A profile is indicative of low risk and type $B$ indicates high risk for coronary heart disease. $H D L$ and $L D L$ profiles were obtained from the same mobility scan meastirement. Gas phase mobility measurements also reveal simultaneously the size distributions of IDL and $\checkmark L D L$ particles in a plasma sample.

\section{Publications}

W. H. Benner, R. M. Krauss, P. J. Blanche, Ion Mobility Analysis of Biological Particles, LBL patent disclosure. 


\section{Microprojectile Accelerator for Targeted Biological Transformation}

Principal Investigators: Jian Jin and Abby Dernburg

Project No.: 02027

\section{Project Description}

This project was to develop an acceleration and targeting system to precisely bombard cells or organisms with micron-sized gold particles coated with bioactive substances. Such a device will provide a versatile means to deliver deoxyribonucleic acid (DNA) and other molecules into specific cells or subcellular compartments. By controlling the delivery of individual particles to the recipient tissue under microscopic observation, the placement, density, and deposition of the passenger material will be optimized. Using this technology, we will develop a novel approach to study protein function on a genome-wide scale.

More specifically, using the technologies of aerosol science and automated imaging acquisition/processing, afi automated system will be developed that implants micronsized gold particles coated with biochemical reagents into an array of selectively targeted areas ( $5 \mu \mathrm{m}$ diameter) of cells or organisms. The particles will be accelerated in an air stream at controllable speed up to sonic velocity allowing firm penetration of cell walls but minimal damages to the cells. The amount of bio-chemicals implanted in a target area will be controlled by adjusting the number of micro-particles implanted and the dosage carried by each particles. We will also buitd an automated microscopic imaging system to image and select interested regions from the sample and direct the implantation. Our performance goal will be to control placement to $\pm 5 \mu \mathrm{m}$ to allow selective targeting within cells and organisms. Using this device, we will optimize DNA transformation of the nematode $C$. elegans. This will enable us to express a library of fluorescent fusion proteins in order to identify interesting genes based an the localization of their products.

\section{Accomplishments}

After carefully investigated the ultimate application need of this project, we decided to employ the biolistics as the core technology. Biolistics is a method of transfection that involves the firing of DNA conted micro-particles at high speeds into a specimen. Currently, the most widely used method of transfection involves injection of DNA into a specimen using a needle. However, the needle-based injection method is severely hampered because it is always over-dosed since the injection volume can not be reduced to a single copy level. Additionally, the needle injection is more likely to cause permanent damages to the cells and is a labor intensive and low throughput process. Biolistics provides an alternative to this technique, and has been successfully used in the past as a method of transfection. In this appronch, the micrometer-sized particles, or microcarriers, that are coated with DNA are made of metal, and are fired into the specimen using compressed gas. Current biolistic devices have many limitations, including inability to deliver particles to a preselected area (in the order of few micron) in a precise, controllable and high throughput fashion and without damaging the target. Having a small target area would enable transfection of small and even microscopic specimens. The ultimate goal of this project is to develop a system that is capable of automatically locating target cells or organism of a living system and delivering very low number of copies of genes to the area causing a gentle and highly efficient genetic transformation.

We have designed and built a prototype "gene gun" that is capable of accelerating micro-particles to a desired penetration speed and directing them into a well-defined target region. To make this device capable of "firing bullets on demand", it is necessary to design the bullets. Currently, the bullets are prepared by coating a short piece of plastic tube with solution containing micro-particles. After dried, micro-particles stick on the inner surfaces of the tube. Upon the firing, the shock-wave generated by the quick release of compressed He gases tears these particles off the wall and the followed high speed jet stream washes and accelerates the particles to reach the desired velocity over the length ( 3 inches) of the gun barrel. The particles are confined by the $3 \mathrm{~mm}$ inner diameter of the barrel and final targeted area is defined by a pin-hole placed about 2 $\mathrm{mm}$ above the specimen. While the pin-hole effectively blocks the shock-wave so preventing deadly damages to the specimen, it also significantly slows down the particles before they pass through. Therefore, it is absolutely necessary to search and fine ture discharge pressure and pulse width of the gating valve to reach optimal bombardment condition once a pin-hole size is selected. We have optimized bombardment conditions for 100 and $50 \mu \mathrm{m}$ pin-holes and is working on $25 \mu \mathrm{m}$ pin-hole, and we don't believe we can reach the $5 \mu \mathrm{m}$ precision with current technology.

To characterize this system, we installed it on an inverted microscope, equipped with a charge-coupled device (CCD) camera. That allows easy characterization of actual bombarded region and penetration depth. To demonstrate its feasibility, we then shot live C. Elegans worms with 1.0 micro diameter golden particles coated with DNA 
repairing ligants. Our preliminary data suggested that the implantation was successful and some of worms had their DNA repaired and their off-springs showed restored mobility.

In order to improve its throughput and efficiency, we have added the automated sample handling and imaging capability to the system. Now it is possible to uutomatically scan through a sample plate and let the image processing software identify designated regions and carry out the implantation. The next step of automation is to implement the bullet loading and we will need to directly dispense drops of micro-particle containing solution onto the inner surface of the gun barrel to allow bombardment with high repetition rate. In the meantime, the biologists are conducting more tests on this system in order to figure out the optimal conditions such as DNA concentration and preparation protocol to improve the transformation rate. 


\section{Environmental Energy Technologies Division}

\section{Development of Cool Colored Shingles}

Principal investigators: Hashem Akbari and Paul
Berdahl

Project No.: 01019

\section{Project Description}

This project is designed to stimulate the development of more reflective roofing materials that would mitigate urban heat island temperature buildup and thus reduce the formation of urban smog and the use of air conditioning. Cool roofing products are generally not available for the residential sector. The solar reflectance of asphalt roofing shingles, the predominant roofing material used for residential buildings in the United States, is largely determined by the pigments in the coatings on the roofing granules used to surface the shingles. Since half of the heat from the sun arrives as invisible radiation in the nearinfrared spectrum, it is theoretically possible to create a "cool" shingle of any color by using a color pigment with high infrared reflectivity.

We are investigating how the optical and other physical (size, shape, etc.) properties of pigment particles affect the solar reflectance of roofing shingles. In particular, we are measuring the spectral reflectances and transmittances of coatings with individual pure pigments and thereby deriving the Kubelka-Murk (KM) coefficients, which describe the absorption and scattering strengths of the pigments at each wavelength in the solar spectrum. The spectral reflectance of a coating made with a mixture of pigments can be computed from these KM coefficients.

\section{Accomplishments}

Of the four objectives in this project - (1) collecting data on optical properties of pigment materials; (2) studying spectral reflectance and absorptance of coatings containing pigment particles; (3) studying spectral reflectance of coated granules; and (4) studying spectral reflectance of asphalt shingles covered with coated granules - the pigment characterization (Objective 2) received the primary emphasis.
We initially collected some data on fundamental optical properties of pigments. We then measured the spectral optical properties of a large catalog of single-pigment paints, including $20 \mathrm{cool}$ and conventional paints manufactured by our industrial partners (Ferro, BASF, and Shepherd Color) and 32 other generic colors. Three samples were prepared for each paint: a free film, a film over opaque white, and a film over opaque black. Freefilm diffuse transmittance, free-film diffuse reflectance, and diffuse reflectance over black and over white were measured as a function of wavelength across the solar spectrum. Diffuse absorptance was computed by subtracting the sum of the transmittance and reflectance from unity. We developed software that performs detailed a mathematical analysis of these measurements, yielding the pigments' "Kubekla-Munk" optical scattering (S) and absorption (K) (see Figure la).

Our algorithm exploits the presence of more film measurements (three reflectances, one transmittunce) than unkrowns pigment properties (two coefficients) to gauge the error in the derived $S$ and $K$ coefficients. When we determined pigment properties from two of the four measurements (e.g., reflectance over white and reflectance over black), we generally found good agreement between measured and computed values of the remaining two film properties (e.g., free-film reflectance and transmittance) (see Figure 1b).

Many of the cool dark pigments identified by this project are mixed metal oxide pigments such as $\mathrm{Cr}_{2} \mathrm{O}_{3}: \mathrm{Fe}_{2} \mathrm{O}_{3}$, often doped with additional oxides such as $\mathrm{TiO}_{2}$ and $\mathrm{Al}_{2} \mathrm{O}_{3}$. The simple green pigment, $\mathrm{Cr}_{2} \mathrm{O}_{3}$, while often identified as infrared reflective, in fact was inferior to mixed metal oxide substitutes. Organic pigments identified as suitable for cool conting formulations include the phthalocyanine blues and greens, and quinacridone red.

Many coatings tend to be translucent in the near infrared. Therefore, we have determined that adequate coating opacity and/or an infrared-reflective substrate are required for optimum coating performance.

For the remaining two objectives, we collaborated with a granule manufacturer (ISP Minerals) to compare the reflectances of asphalt shingles surfaced with cool colored granules to the reflectances of smooth films made with the same granule coatings. We found that the shingles typically had only half the reflectance of the smooth paint films because the color coatings on the granules were so thin as to be infrared-transparent. The roughness of the asphalt shingles also reduces their reflectance. 
During the second and final year of this project, we applied for and received funding from the California Energy Commission for a new, multi-year project on research, development, and demonstration of cool colored roofing materials. This project is in collaboration with
Oak Ridge National Laboratory, and involves pigment, roofing granule, and roofing manufacturing companies. Our efforts to characterize and optimize the performance of granules and shingles will be continued in the new project.
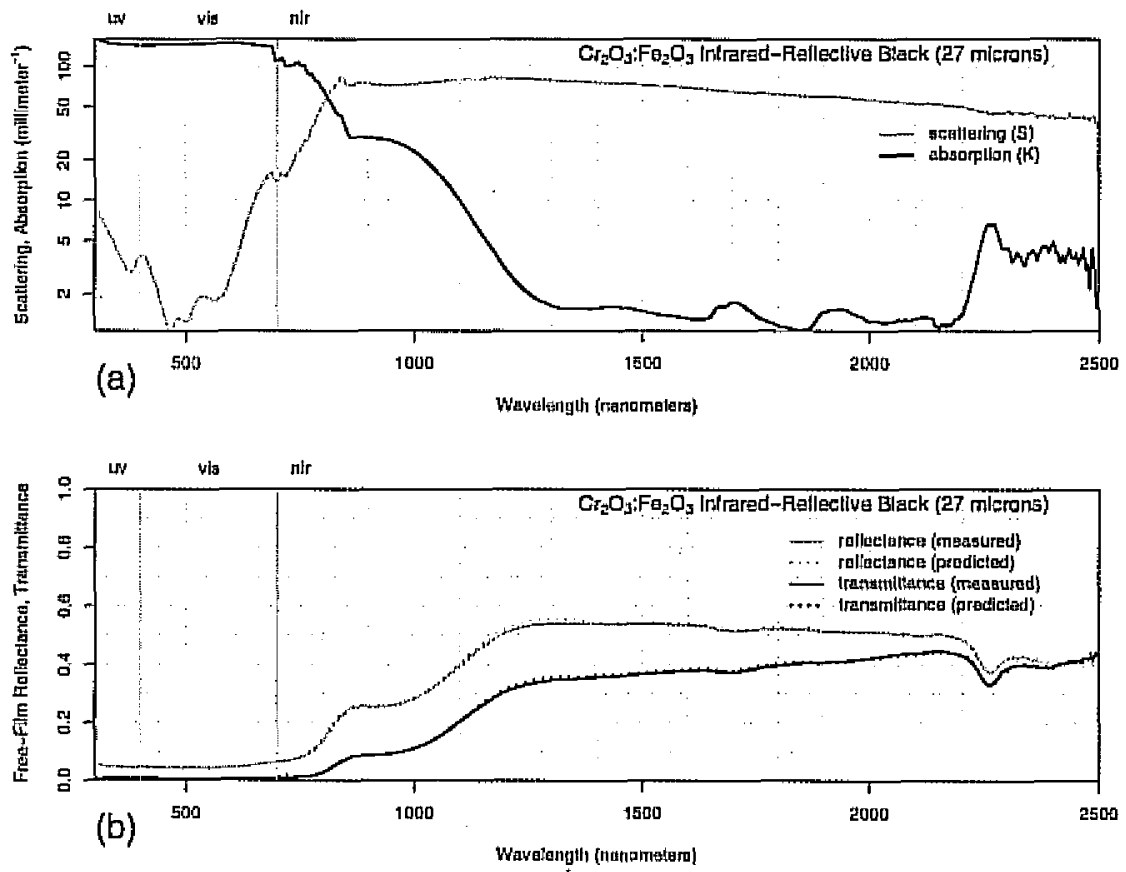

Figure 1: Optical properties of an infrared-reflective black paint. Shown are (a) calculated Kubelka-Munk, scattering and absorption coefficients based on the reflectances of a 27-micron thick film meastured over white and black backgrounds, and (b) the measured and predicted transmittances and reflectances of a 27 -micron-thick free film.

\section{Publications}

R. Levinson, P. Berdahl, and H. Akbari, "Solar-Spectrum Optical Properties of Pigments for Cool Roofing Materials," draft paper in preparation.

H. Akbari and P. Berdahl, "Cool Colored Roofing Granules," Invention disclosure, IB 1722P, September 2001, patent application filed October 22, 2002..

\section{Miniaturized Systems for Particle Exposure Assessment}

Principal Investigators: Michael Apte, Lara Gundel, and Anthony Hansen

Project No.: 02028

\section{Project Description}

This project is developing prototype instrumentation to advance knowledge about the relationship between exposures to particles and human health, a national research priority. At present, statistically-adequate studies of relationships between health outcomes and particle 
concentrations and composition are prohibited by high cost and limited capabilities of suitable instrumentation.

Using advanced sensor technology, we are prototyping the next generation of low-cost compact instrumentation for particulate matter (PM) exposure assessment. The Miniaturized System for Particle Exposure Assessment (MSPEA) will measure aerosol mass, size and composition simultaneously.

In this project we are attempting to utilize a low-inertial particle collection mechanism that has been under-utilized in existing particle measurement technologies. The physics of this mechanism scale well with microelectromechanical mass measuring devices such that the particle mass sensor can be integrated into very small packages-potentially to the scale of microchips.

The optical properties of the most abundant and harmful airborne particle species (i.e., diesel exhaust, environmental tobacco smoke, and woodsmoke) are well understood, enabling us to design simple optical probes that can differentiate between them when they are collected on a reflective or transparent surface. Advances in microelectronic light sources and detectors, and fiber optics will enable us to integrate miniature optical probes into the miniature particle mass monitor.

\section{Accomplishments}

The success of this work has led to submission of a record of invention. The proprietary nature of the inveniion precludes disclosure of the technical details of the MSPEA sensor. Thus the discussion below is presented in broad terms.

In the first year, we tested four major components of the MSPEA sensor:

- An acoustic wave device (AWD) was used as real-time mass sensor and particulate matter (PM) deposition surface;

- A low inertial particle collection (LIPC) mechanism that precipitates PM fron the air onto the AWD surface, where it is captured by VanderWaal's forces;

- An optical probe system configured to monitor the AWD surface as particle mass loads it; and

- A low-flow system that provides a monitor inlet size cut for PM10 (rejecting PM with aerodynamic diameter $>10 \mu \mathrm{m}$ ) or PM2.5 (rejecting PM with aerodynamic diameter $>2.5 \mu \mathrm{m})$, based upon sizedependent characteristics of particles.

We performed tests to optimize the LIPC. We identified suitable collection characteristics with sensor configurations on the 1-millimeter scale. Chamber experiments with high concentrations of environmental tobacco smoke (ETS) yielded a sharp AWD response, with collection of about $80 \mathrm{ng}$ of particles on the AWD substrate within a few minutes. Visual inspection of the AWD surface showed a distinct "track" of particle deposition on the substrate surface (see figure). Identical tests with the LIPC turned off yielded no mass changes and no observed deposit. Chamber tests show that the AWD signal tracks PM concentration data collected using an optical particle counter very well, as evidenced in the figure.

We used a commercially available optical reflection/backscattering probe to show that it is possible to measure the change in absorbance of the AWD surface at two wavelengths as a function of particle loading. The probe was positioned about $2 \mathrm{~mm}$ above the surface of the AWD. The AWD reflection was strongly attenuated at one light wavelength due to the characteristic absorbing properties of the ETS, while the reflectance at a different wavelength was only slightly attenuated (see Figure 2). This pattern is expected for ETS. Although not yet fully verified for the MSPEA system configuration, it is expected theoretically, and has been shown experimentally in other optically-based instruments, that diesel exhaust $P M$, a black inorganic carbon aerosol, would absorb equally at both of these wavelengths. Furthermore, other PM species would respond with different ratios of absorbance at these wavelengths. Although not fully integrated into the device yet, our results prove the concept of integration of a real-time reflectance probe used to measure optical attenuation due to absorbance. The commercial optical equipment discussed above will easily be replaced by off the shelf opto-electronics. Optical fibers can be configured to pipe the light to and from the sample substrate.

The upper size bound of the aerodynamic diameter size distribution of particles collected by the device can be controlled to match current Environmental Protection Agency (EPA) reference method inlet cut sizes of PM10 and PM2.5 using a method discussed in our record of invention. Using this method, and by adjusting the inlet tube diameter and sampling flow rate, it is possible to achieve the desired cut-point size. Size cut data were not collected using the miniaturized geometry in this first year. 


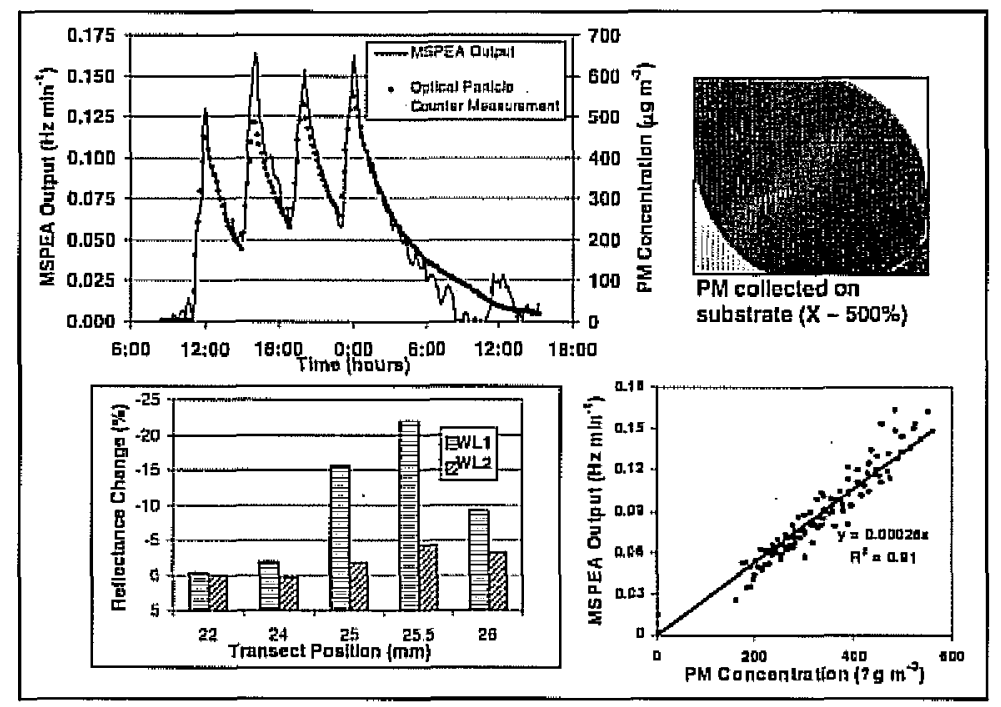

Figure 2: Figure of MSPEA sensor testing results during Year 1 of LDRD project. Shown is a real time plot of particle matter concentration ws. time during smoking of four cigarettes in an environmental chamber (upper left) for MSPEA and an optical particle counter (OPC). The OPC and MSPEA data are also plotted against each other (lower right). PM loading can be seen visually on blown up pltotograph of the MSPEA sampling substrate (upper right). Optical probe response at two wavelengths to environmental tobacco smoke on loaded pait of substrate is shown (lower left).

\section{Publications}

M.G. Apte, L.A. Gundel, A.T. Hansen and D.R. Black, Miniaturized System for Particle Exposture Assessment, Record of Invention.

\section{Simulation and Interpretation of the Effects of Transmission Congestion on Restructured Wholesale Electricity Markets}

Principal Investigators: Joeseph Eto and Chris Marnay

Project No.: 02046

\section{Project Description}

The purpose of this L.DRD is to initiate development of an integrated suite of analytic tools, data, and methods for assessing the national significance of transmission bottlenecks, and of options to address them. We hypothesize that supercomputing may be an essential tool for enabling better analysis of transmission bottlenecks and will devote special attention to assessing the improvements that could be made possible by introduction of supercomputing techniques to analyze transmission system bottlenecks.

Three tasks will be undertaken. First, available power flow and economic models will be reviewed and their suitability for enhancement evaluated. Second, an analysis of congestion contracts in the New York region will be conducted. And third, a research plan for expanding the performance of power flow tools will be developed.

\section{Accomplishments}

Our research suggests that detailed economic and electric models are required to accurately assess critical transmission bottlenecks in the nation's electric power system. The extensive economic and engineering models and tools needed to properly and completely address the problem requires further development that will benefit from specialized software expertise and supercomputing facilities.

Recent compelling reports, including the National Transmission Grid Study, show significant differences in electricity prices across regions of the country due to deficiencies in the nation's transmission infrastructure. The transportation-type models used in such studies clearly identify important economic centers and point to congested interfaces that inhibit beneficial trade between centers. These models do not claim to, and cannot, identify the specific location and importance of physical limiting apparatus. However, one might erroneously infer that 
limiting constraints occur at interfaces between areas. Since these models use a highly simplified and aggregated model of the physical network, more detailed studies are employed to identify individual limiting components.

Detailed engineering models show that a number of different constraints limit favorable transfer between areas including the limited capacity of transmission lines connecting areas. It is also common for internal voltage constraints to be the primary limiting factor. In some cases the power transfer between neighboring areas is limited by distant constraints outside both of the areas contractually involved. To conduct a comprehensive study of the interface between two areas requires a model detailing those areas and far beyond. In addition to the results of these models, empirical studies of market data reveal that the precise time and location of congestion in the network is difficult to predict. New York Independent System Operator (ISO) transmission congestion contracts show that traders failed to accurately predict congestion. The temporal and spatial volatility of congestion remains poorly understood.

A study of bottlenecks on the national level will benefit from the ability to analyze detailed models of each of the three synchronized interconnected grids. The eastern interconnect, essentially comprising the states east of the rocky mountains excluding Texas, has a nominal power flow model with approximately 35,000 buses, 45,000 lines, and more than 5,000 generators. It is a nonlinear model requiring specialized to techniques for evaluation. In addition to the analysis of a nominal system model, it is necessary to evaluate the system under a myriad of limiting conditions to account for the absence of equipment, due to maintenance and unplanned outages. The size of the model and the vast number of operating conditions to consider make a thorough analysis challenging.

A detailed electric model is not enough. To identify critical transmission bottlenecks we need an accurate economic model to evaluate the financial impact of congestion and relief. To this end we suggest that a repeated auction market model simulation is required. We recognize that enhancements to the grid will alter the behavior of the market. We suggest that a market simulation with software agents representing participants will enable a more accurate analysis of the benefits of transmission expansion. The value of the market simulation will increase with the inclusion of a technique to identify cases in which market manipulation by participants is possible. Reducing the possibility of market manipulation through transmission expansion, and other means, will eliminate unintended artificially inflated electricity prices. Presently available commercial electricity market simulation tools do not use detailed electric system models and cannot be used for our purposes at this time. Custorn software development is in warranted.
To design an electricity market structure that stimulates efficient transmission investment, it is absolutely necessary to understand and predict congestion. Therefore, we will recommend that further research be initiated to develop a needed tool to unite economic and engineering models to correctly identify the limiting physical conditions associated with critical transmission bottlenecks. The tool will couple an economic market simulation model with a detailed electrical model. It may incorporate the Federal Energy Regulatory Commission (FERC) standard market design with the ability to use software agents to represent market participant behavior, and identify cases of excessive market manipulation. The electric model needs to encompass at least an entire synchronized interconnected area. Such a detailed model will require enormous computing power and will benefit from supercomputing applications.

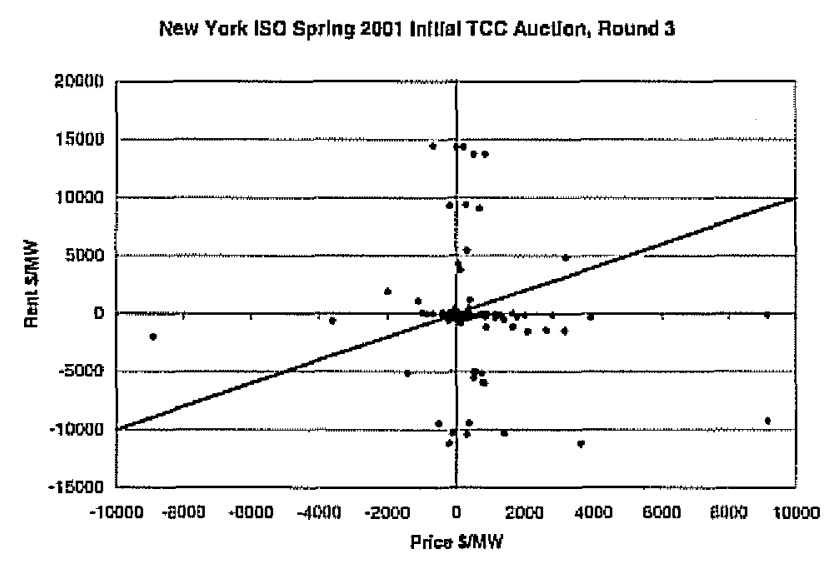

Figure 3: This graph shows results from one particular auction leld in New York; one in which participants should be purchasing congestion rights as a hedge against congestion costs. Points on the 45-degree line shown imply individual contracts whose buyers have correctly predicted congestion. The scattered data points indicate that the market participants have predicted congestion poorly.

\section{Publications}

A.S. Siddiqui, E. Bartholomew, C. Marnay, and S.S. Oren, "The Effectiveness of Transmission Congestion Contracts in Hedging Transmission Congestion Risk in New York State," POWER Conference, March 2003, December 2002. submitted for publication. 


\section{Indoor Bioaerosol Detection and Quantification by Polymerase Chain Reaction (PCR)}

Principal Investigators: William Fisk and Regine GothGoldstein

Project No.: 01020

\section{Project Description}

Indoor airborne bioaerosols contribute to a broad range of health effects including communicable respiratory illness such as influenza and common colds. Measurements of the airborne concentrations and sizes of the virus or bacteria causing communicable respiratory illnesses are critical for research on the relationship of exposures to health effects, for studies of the influence of building characteristics on exposures, and for evaluations of exposure mitigation measures. Measurement techniques have been especially limited for airborne virus, such as human rhinovirus (HRV), which cause approximately half of common colds. Although controlled laboratory studies have indicated that HRV infections are transmitted, in part, through the inhalation route, we have no detailed information on the size of aerosols with HRV. We have only minimal information on the periods during illness when aerosols containing HRV are shed, the airborne HRV concentrations that cause illness, and the potential benefits of increasing building ventilation rates or filtration efficiency. Accordingly, the objectives of this project were to develop and demonstrate a quantitative polymerase chain reaction (PCR) assay for airborne HRV, as an alternative to current onerous sampling and analysis methods, and to use this assay to study the size distribution human-produced airborne particles containing rhinovirus.

The approach to this work is encapsulated within the following five tasks. Task 1 was the development and validation of the quantitative $\mathrm{PCR}$ assay for rhinovirus, using published primers to amplify a conserved region of the rhinovirus genome. The PCR target was quantified with the real-time PCR Lightcycler system. The quantitative performance of the assay was determined with a quantitative standard (HRV QS) obtained by cloning a 425 base-pair fragment of the HRV genome into a bacterial cell line. In Task 2, we determined whether the assay could detect HRV in nasal lavage samples from subjects with common cold symptoms and quantified the amount of HRV in the samples. Task 3 selected a sampling system for aerosals that could contain HRV and developed and tested a means of recovering HRV from the collected samples. Recovery efficiency was determined using samples spiked with known quantities of HRV. In Task 4, we determined whether the sampling process and assay, used together, could detect an artificially-produced aerosol containing HRV obtained from a nasal lavage sample. Finally, in Task 5 volunteers with cold symptoms worked for approximately two hours in a ventilated chamber while samples of aerosols in multiple size ranges were collected from within the chamber. The quantitative PCR assay was used to analyze the samples for HRV.

\section{Accomplishments}

For the first task, we were successful in developing quantitative PCR assay for HRV. The assay has a linear response over a 10,000 -fold range in mass of HRV with a variance of $8 \%$ at the $5000 \mathrm{fg}$ level and $20 \%$ at the $50 \mathrm{fg}$ level (see Figure 4). A quntitation limit of $5 \mathrm{fg}$ of HRV QS was observed, below this level quantitation is no longer valid.

For the second task, after completing testing of the assay, nasal lavage samples were collected from six volunteers with head cold symptoms and one volunteer with allergy symptoms who were recruited during a 1 month period in the summer. Four of the six head cold specimens were found to be HRV positive (an HRV incidence rate of 57\%) while the specimen from the allergy sufferer was negative and served as a negative control. The concentrations of $\mathrm{HRV}$ in 4 of these positive lavage specimens ranged from $0.2 \mathrm{pg} / \mathrm{ml}$ to $21 \mu \mathrm{g} / \mathrm{ml}$ of lavage fluid. To the best of our knowledge, this was the first-ever quantification of HRV in such samples. Thus, we determined that the assity could detect and quantify HRV in nasal lavage samples.

For collection of samples from air, we selected a six-stage viable cascade impactor with $50 \%$ size cuts of $7,4.7,3.3$, 2.1, 1.1 and 0.65 microns. A $45 \mathrm{~mm}$ diameter teflon-coated glass fiber filter was used as a final stage (to collect particles with sizes $<0.65$ microns). Samples were extracted from the collection surfaces using a lysis buffer. Through analyses of samples spiked with known amounts of HRV, we determined that $60 \%$ of the HRV was recovered with a variance of $30 \%$. Initial experiments with the HRV QS show that the FRV quantitation standard was not degraded during the sampling process with the six-stage impactor

To verify that the sampling and analysis process was suitable for airborne HRV, we measured an HRV aerosol (artificially produced in a sealed chamber) using the cascade impactor to collect airborne particles. A strongly positive nasal lavage specimen was diluted to a final concentration of $6 \mu \mathrm{g} / \mathrm{ml}$ of HRV. A total volume of $3 \mathrm{ml}$ $(18 \mu \mathrm{g} \mathrm{HRV})$ was released into a $27 \mathrm{~m}^{3}$ chamber then 2.2 $\mathrm{m}^{3}$ of air was sampled with the impactor. Each stage of the 
impactor was analyzed for HRV by the quantitative assay. We were successful in detecting and quantifying the HRV in the collected samples.

For the final task, after developing and validating the HRV assay, seven volunteers exhibiting head cold symptoms worked for approximately two hours in our research chamber while we collected samples of particles from the chamber air with the multi-stage impactor. Based on analyses of nasal lavage samples, five of the volunteers were negative for HRV (an incidence rate of $29 \%$ ). The two HRV positive volunteers, who displayed only light symptoms (some coughing and no sncezing), were weakly positive for HRV by nasal lavage. However, no HRV could be detected on any stage of the impactor. Thus, to date we have not been able to quantify the size distribution of airborne particles with HRV. Our calculations show that HRV viral production in the nasal mucosa of infected individuals is great enough to suggest the feasibility of detecting HRV in acrosol samples. We measured the viral load in one nare of an individual of approximately $1 \times 10^{7}$ HRV genome equivalents. Taking into account the assay sensitivity and the sampling and recovery efficiencies, we need to collect $3 \times 10^{3}$ HRV organisms or $0.03 \%$ of the viral load in one nare. With one completion of this LDRD project, we wilt seek other support to enable studies with future subjects.

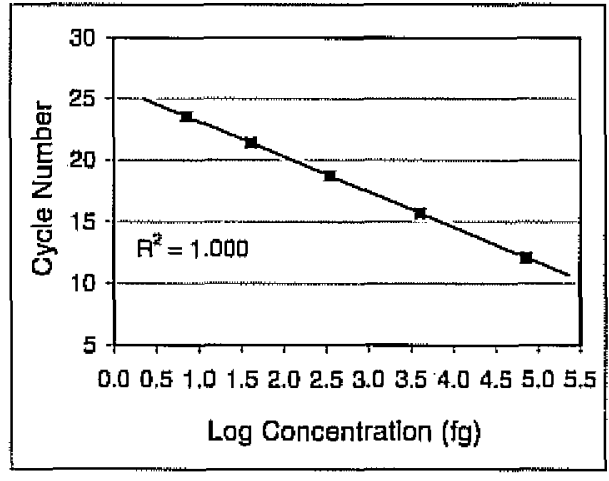

Figure 4: Standard curve for quantitation of $H R V$ by $P C R$ assay developed in this LDRD project.

\section{Publications}

M.L. Russell, R. Goth-Goldstein, M.G. Apte, and W.J. Fisk, "Method for Measuring the Size Distribution of Airborne Rhinovirus," Indoor Air 2002, Ninth International Conference on Indoor Air Quality and Climate, vol., pp. 40-45, Indoor Air 2002, Inc., Santa Cruz, CA, June 30 - July 5, 2002.

M.L. Russell, R. Goth-Goldstein, M.G. Apte, and W.J. Fisk, "The Size Distribution of Airborne Rhinovirus," Draft completed, awaiting final data to be submitted to Indoor Air Joumal.

\section{Health Effects of Indoor and Outdoor Particle Concentrations, Assessed with Epidemiology}

\author{
Principal Investigators: Mark Mendell
}

Project No.: 02029

\section{Project Description}

The scientific goals of this project are (1) to advance scientific knowledge about the health effects of particles, by evaluating the association of respiratory symptoms with exposures to indoor and outdoor particle concentrations and determining the protective effects of filtration systems; and (2) to evaluate the feasibility of conducting an innovative epidemiologic study to assess health effects of outdoor particles separately from those of outdoor gaseous pollutants, and also to assess the benefits of enhanced filtration in protecting a susceptible population against outdoor particles.

This study will contain two main elements. The first element focuses on analysis of a unique epidemiologic data set from 100 US office buildings, to determine if respiratory symptoms are associated more strongly with indoor than with outdoor concentrations of particles, and more with fine than with coarse particles. The second element involves evaluating the feasibility of several innovative epidemiologic studies to assess health effects of indoor exposures to particles originating outdoors. These studies use differences in air filtration to isolate effects of particle and gaseous pollutant exposures and to quantify benefits of higher efftciency filtration for susceptible populations. Evaluated designs will include a large observational follow-up study of major health events, in multiple facilities with differing protection of occupants from outdoor particulate pollutants, and a smaller intervention study assessing the effects on clinically monitored health parameters of improved protection from particulate pollutants in residential buildings.

\section{Accomplishments}

In our analyses thus far of data collected by the US Environmental Protection Agency (EPA) from a representative set of 100 office buildings, we have found that concentrations of fine and coarse particles, both inside and outside the buildings, were low. Concentrations of particles, inside or outside, were not related to lower respiratory or mucous membrane symptoms among the occupants. On the other hand, we have discovered two 
surprising and inexplicable relationships in our data, within the preliminary statistical models we have constructed thus far. The more efficient the particle filtration in the heating, ventilation and air-conditioning (HVAC) system of the buildings studied, the more frequently the occupants reported some health symptoms within the building. For instance, in buildings with the most efficient filters compared to those with the least efficient filters, occupants were about $80 \%$ more likely to report lower respiratory symptoms (wheeze, tight chest, shortness of breath, or cough) and about $45 \%$ more likely to report neurologic symptoms (headache, fatigue, or difficulty concentrating). Also, occupants within buildings where particle filters are changed more often (monthly vs. less) were over $50 \%$ more likely to report symptoms of eye, nose, or throat irritation within the building. We are continuing to explore whether these relationships are due to statistical confounding by other related factors not yet included in the model.

In our evaluation of the feasibility of studies to assess health effects of indoor exposures to particles originating outdoors, we have now defined four different approaches for consideration. Three are epidemiologic field studies, and one is a statistical analysis and modeling project. The three field study approaches are: to study a large number of skilled nursing facilities for the elderly, using measurements of how much the buildings shelter the occupants from outdoor particles, along with routinely collected health outcome data; to study a small number of susceptible subjects, such as the elderly or asthmatics, to compare how much their residences shelter them from outdoor particles with some physiologic measure of a health effect, possibly including interventions to increase the sheltering for some subjects; and to study intensively several subjects previously documented to have a physiologic response to outdoor particle concentrations, to see if removing fine particles from their indoor environments reduces their response to the outdoor concentrations. The statistical modeling approach would involve estimating, based on available statistical tools, corrected health risk estimates for the effects of outdoor fine particles, by correcting the estimated errors resulting from use in risk models of particle concentrations measured at central sites outdoors in each region, while exposure to these particles for most individuals actually occurs indoors, in buildings with a varying degrees of sheltering from outdoor particles.

Thus far, we have ruled out the statistical modeling approach, because it does not seem likely to lead to further research questions, and we have ruled out the large field study in nursing homes, because the required health outcome data is not routinely collected in these facilities. We are collecting more detailed information about the other two field study approaches, to assess their feasibility. 


\section{Life Sciences Division}

\section{Quantitative Spatial and Temporal Resolution of Multicellular Interactions}

Principal Investigators: Mary Helen Barcellos-Hoff, Damir Sudar, Bahram Parvin, Abby Dernburg, Daniel Callahan, and Richard Schwarz

Project No.: 01021

\section{Project Description}

The purpose of this collaborative interdivisional project was to provide an integrated resuurce for imaging of biological models in context, i.e. systems biology. Quantitative imaging of complex biological systems is a critical challenge for understanding how the expression of the genome results in 300 coordinated multicellular behaviors in the human body. Using genomic information to understand the biology of complex organisms requires comprehensive knowledge of the dynamics of phenotype generation and maintenance. A phenotype results from selective expression of the genome, creating a history of the cell and its response to the extracellular environment. Several thousand antibodies and reagents exist for differentiating a cell's specific protein components. Some antibodies can also discriminate between functional variants of a protein caused by modifications such as phosphorylation status, protein conformation, and complex formation. Inherent biological variability and genomic instability are additional factors that support the requirement for large-population analysis. Defining cell phenomes requires tracking the kinetics and quantities of multiple constituent proteins, their cellular context, and their morphological features in large populations. Such studies should also include responses to stimuli for use in generating and testing functional models.

Current sequential measurements obtained with various microscopy techniques preclude detailed analysis of multidimensional responses. Quantitation of spatial and temporal concurrent behavior of multiple markers in large populations of multicellular aggregates is hampered by labor-intensive methods, a lack of quantitative tools, and the inability to index information. Ideally, one would track the kinetics and quantities of multiple target proteins, their cellular context, and morphological features in large populations. Future innovation is needed at the intersection of microscopy, all biology data acquisition strategies, image analysis, and high speed computing.

\section{Accomplishments}

The BioSig imaging bioinformatic system for characterizing phenomics (http://vision.lbl.gov/Projects/BioSig) is a result of the Parvin and Barcellos-Hoff ongoing collaboration. This system provides a data model for capturing experimental annotations and variables, computational techniques for summarizing large numbers of images, and a distributed architecture that facilitates distant collaboration. Recent accomplishments include Callahan and Parvin's refinement of a data model for capturing the dynamic changes in the micro-environment visuo-servoing optical microscopy studies and development of a new graphical interface using Scalable Vector Graphics (SVG) technologies for annotation of experimental variables in BioSig.

As a result of the requirements for analyzing multicellutar confocal images from the Barcellos-Hoff laboratory, Parvin has developed additional methods for automatic detection of cell structures and localization of protein expression from a volumetric dataset. Detection of an individual nucleus reveals morphological features like size and shape, can be used to map the multicellular organization, and enables localization of intercellular signaling components as a function of treatment. Automated analysis and counting of can be hampered by abundant speckle noise, which frequently has a signature similar to target proteins in the volumetric dataset. The detection of other features, in this case individual nuclei provides the necessary "context" to filter speckle noise and enables automatic counting and characterization of target protein expression (see figure). A software tool for calibrating the fluorescence intensity and its leakage at multiple excitation frequency coupled with a cooled charge-coupled device (CCD) camera has been developed as well.

In microscopy, Sudar has implemented the use of structured light to perform optical sectioning. A piezo-mounted Ronchi grating located in the field diaphragm of the epifluorescence microscope permits the acquisition of three images with the grating period shifted by $1 / 3$ each. A simple linear calculation yields an optically sectioned image with the out-of-focus information highly attenuated. Structured light shares many of the benefits of restoration microscopy over confocal microscopy such as low photodamage or bleaching and white light excitation resulting in high flexibility in fluorochrome choice. 
The biological research questions that can benefit from this technology include understanding the effects of ionizing radiation, mapping proteins during fly development, studying chromosome segregation in worms, and monitoring cancer cells response to chemotherapy drugs. The ability of investigators to advance in biology is enhanced by state of the art imaging expertise and novel imaging strategies. Implementation of new imaging methodologies and development of novel imaging algorithms-in conjunction with current Berkeley Lab capabilities in visual servoing, high-speed recording, and analysis - allowed the development of essential capabilities for simultaneous quantitative analysis of large multicellular populations. Computational modeling of the resulting biological data may ultimately provide hypothesisgenerating analysis of complex responses and provide a basis for predictive modeling in terms of risk and human health.

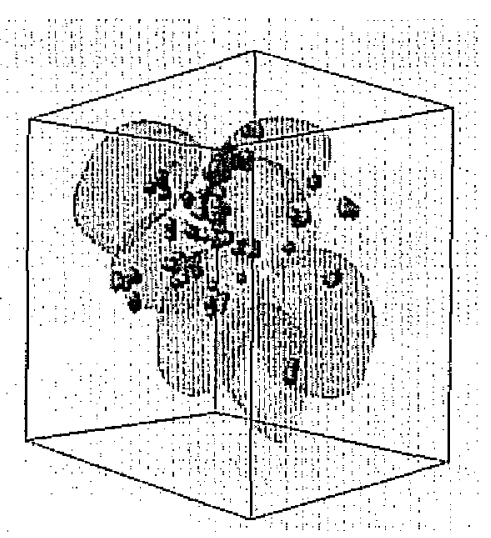

Figure 1: Localization using 3D context image analysis of junctional complexes (green) inaged by confocal microscopy in an aggregate of cultured cell nuclei (shaded).

\section{Publications}

C. Ortiz De Solorzano, S. Costes, D.E. Callahan, B. Parvin and M.H. Barcellos-Hoff, "Applications of Quantitative Digital Image Analysis to Breast Cancer Research," Microscopy Research and Techniques, 2002, 59: 119-127.

B. Parvin, Q. Yang, G. Fontenay, and M.H. Barcellos-Hoff, "BioSig: An Imaging Bioinformatic System for Studying Phenomics," IEEE Computer, 2002, 35: 65-71, vision.lb.gov, under publication.

Q. Yung, B. Parvin, and M. H. Barcellos-Hoff, "CHEF: Convex Hull and Eliptic Features for Detection of Blobs in Biological Images," International Conference on Pattern Recognition, Quebec City, Canada, August 2002, vision.lb.gov, under publication.
B. Parvin, Q. Yang, G. Fontenay, and M.H. Barcellos-Hoff, "An Imaging Bioinformatic System for Phenotypic Studies," IEEE Trans. On System, Man, Cybernetics, in press.

\section{Positron Emission Tomography (PET) Ligands for the $\mathrm{N}$-methyl-D-aspartate (NMDA) Receptor Channel}

Principal Investigators: Anat Biegon

Project No.: 01022

\section{Project Description}

The ultimate purpose of this project is to develop and validate a new brain imaging agent which would be used to optimize and monitor treatment of stroke and brain injury and to provide new insights into the etiology and treatment of addictive behavior and other brain disorders linked to increased or decreased activity of glutamate, the major excitatory neurotransmitter in the brain. The immediate goal is to synthesize, screen, and optimize new radiopharmaceuticals suitable for $i n-v i v o$, non-invasive imaging of activated brain glutamate NMDA receptors by positron emission tomography (PET).

New molecules suitable for labeling with positron emitters (Fluorine-18, Carbon-11) will be synthesized from lead compounds and screened by in-vitro binding methods for improved affinity and lipophilicity. Specificity to the NMDA receptor will be assessed by a combination of invitro binding and autoradiographic techniques. In-vivo selectivity and kinetics will be evaluated by autoradiography and biodistribution studies. The best molecule will be labeled with Carbon-11 or Fluorine-18, and investigated in dogs for biodistribution, dosimetry, and safety. Two-species subacute toxicology will be evaluated with the "cold" compound if necessary, in preparation for evaluation in humans.

\section{Accomplishments}

\section{Chemical Synthesis and In Vitro Screening}

Fluorine-containing derivatives of Cerestat (candidates for F-18 labeling): 4 new molecules were synthesized and purified, the affinity of screened molecule was lower than that of CNS5161. 
Candidates for C-11 labeling: 4 new molecules (ethers, iodo and bromo derivatives) of CNS5161 were synthesized and purified. Screened molecules had Kds in the 5-100nM range. Thus, CNS5161, with a Kd of $2-3 \mathrm{nM}$, is the best candidate for radio-labeling.

Radiochemistry: Significant advances have been made towards preparing a precursor for single -step radiolabeling with Carbon-11.

\section{In Vivo Studies}

Intravenous injection of [3F]CNS5161 in untreated animals resulted in a reasonable brain uptake and a regional distribution reminiscent of the distribution of NMDA receptors. Animals treated with NMDA to enhance channel opening had higher region/cerebllum ratio and the distribution (high in hippocampus and cortex, lower in striatum, lowest in cerebellum and white matter) was a close match to NMDA receptor distribution.

\section{Publications}

A.R. Gibbs, H. Morimoto, H.F. VanBrocklin, P.G. Williams and A. Biegon, "Synthesis of N-(2-chloro-5methylthiophenyl)-N'-(3-methyl-thiophenyl)- $\mathrm{N}^{\prime}-$ $\left[{ }^{3} \mathrm{H}_{3}\right]$ methylguanidine, $\left.\left[{ }^{3} \mathrm{H}_{3}\right] \mathrm{CNS}-5161\right]$, , Journal of Labelled Compounds and Radiopharmaceuticals 45:395400 (2002).

A. Biegon, S. Hanrahan, M. Ono, and M. Alvarado, "Development of CNS-5161 for NMDA Receptor Imaging," Journal of Nuclear Medicine, June 2002.

J M. Gerdes, P.A. Wilson, and A. Biegon, ${ }^{+C}$ Computational Studies of 3-D Molecular Similarity by Ligand Superposition: Generation of a Pharmochore Model of NMDA Noncompetitive Inhibitors," Journal of the American Chemical Society (ACS), March 2002.

D.B. Bolstad, P.A. Wilson, J.M. Gerdes, A. Gibbs, and A. Biegon, "Ligand Studies for the NMDA Receptor channel: Synthesis and Binding Profiles of N-(2-chloro-5thiomethylphenyl)- $\mathrm{N}^{\prime}$-(3-substituted-phenyl)-N' methylguanidines," Journal of the American Chemical Saciety (ACS), March 2003, submitted for publication.

A. Biegon, M. Alvarado, M. Ono, S.E. Taylor, and H.F. VanBrocklin, "A Novel Tritiated Non-competitive NMDA Antagonist $\left[{ }^{3} \mathrm{H}\right] \mathrm{CNS}-5161:$ In Vitro and In Vivo Characterization," in preparation.

\section{A High-sensitivity In-Vivo Crosslinking Method}

Principal Investigators: Mark Biggin

Project No.; 01023

\section{Project Description}

Metazoans are distinguished from microbes by their extraotdinarily complex arrangements of cells. The differences between cells within an animal are fundamentally differences in gene expression; and one of the most prominent levels at which gene activity is regulated is at the level of ribonucleic acid (RNA) transcription. Sequence-specific deoxyribonucleic acid (DNA) binding proteins, anyone of which can bind tightly to only a limited number of DNA sequences, are the principle agents used to selectively control gene transcription. Our long-term goal is to characterize the range of DNA sequences bound by transcription factors in animals, the forces that determine the range of DNA sequences bound, and the function of factors bound to their targets. This goal is challenging for many reasons: for example, animal regulatory networks are vastly more complex than those of microbes; metazoan promoters and genomes are much longer; and many metazoan factors recognize shorter, more degenerate DNA sequences. All of which makes it impossible to predict the in-vivo pattern of DNA binding from simple measurements of in-vitro DNA affinities.

To overcome the above challenge, we previously developed a method that directly measures relative occupancy of specific factors on DNA in intact, living animals. We have examined in-vivo binding of the homeoprotein family of developmental regulators in the fruit fly Drosopirila melanogaster as this is probably the most experimentally tractable system available: Drosophila is an outstanding model organism for genetic and transgenic analysis; the developmental regulatory cascade initiating development is best understood in this organism; and the complete genome sequence is available-allowing system-wide studies to be conducted. Our results indicate that the pattern of homeoprotein DNA binding differs dramatically from that predicted by indirect experiments. These proteins bind in a surprising manner to sites throughout the length of most genes. Further, complementary genetic experiments indicate that each homeoprotein regulates the expression of a majority of genes; and a variety of molecular data imply that most of these genes are directly controlled. Our work aims to better characterize the above observations and to determine how transcription factors that bind so broadly 
can coordinate complex and precise events such as morphogenesis.

There are two limitations of our current in-wivo DNA binding data. First, we can only measure interactions to 0.5 to $2 \mathrm{~kb}$ regions of DNA that contain 5 to 15 recognition sites for a specific factor. Thus, we cannot be certain which particular elements are occupied, hampering our ability to determine the functional significance of the widespread DNA binding we observe. Second, we can only measure binding to one DNA fragment at a time. It would be much better if we could look at binding to thousands of DNA regions simultaneously. This project seeks to overcome these two problems.

\section{Accomplishments}

In the first year, we set up a genomic microarray in-vivo crosslinking method that can measure binding to thousands of one $\mathrm{kb}$ genomic fragments at one time. This work was done in collaboration with the Berkeley Drosophila Genome Project as part of a large multi laboratory collaboration that Mark Biggin is organizing to study the transcriptional network in the early Drosopltila embryo. We have now further increased the sensitivity of the assay and shown that the microarray method is quantitative. We have also adapted an affinity capture resin for use in this procedure that works to higher efficiency than those used previously and can analyze larger DNA fragments. We have also set up a microarray data base to analyze our results. We will continue to optimize the sensitivity of our protocol and are working with Affymetrix to design a high density array that covers a larger portion of the Drosophila genome.

\section{Dynamic Reorganization of Chromosome Architecture During Meiosis}

\section{Principal Investigators: Abby Dernburg}

Project No:: 01024

\section{Project Description}

All sexually reproducing organisms rely on a special cell division process called meiosis to generate gametes, which contain only a haploid number of chromosomes. The central event of meiosis is a reductional division in which pairs of homologous chromosomes must segregate to different daughter nuclei. The accuracy of this unique division depends on recombination, the presumed raison d'être of the entire process, which in turn requires that each chromosome must find and pair with its appropriate homologous partner. Errors in mejosis lead to missegregation, with disastrous consequences if an embryo inherits an abnormal number of chromosomes.

Meiosis presents a dramatic example of large-scale nuclear reorganization in response to a specific differentiation program. Many aspects of this process are still mysterious, with no clear precedent in our molecular understanding of biology. How do chromosomes first encounter their partners? How does each recognize its homolog? How do they achicve side-by-side alignment along their entire lengths? How does the protein scaffold called the synaptonemal complex assemble between partner chromosomes? Within this structural context, what regulatory mechanisms ensure that each chromosome undergoes reciprocal recombination while placing tight restrictions on the number and placement of crossovers? How are these nuclear events coordinated with the developmental program giving rise to sperm, oocytes, spores, or pollen? The mechanisms used by the meiotic cell to orchestrate all of these complex processes are virtually unknown.

We will study the complex macromolecular structures that are built and disassembled during meiosis. We will harness both the molecular genetic and cytological advantages of the tiny soil-swelling nematode $C$. elegans. This integrated approach will bridge the gap between individual gene products and large-scale meiotic structures.

The long-term goal of this project will be to understand the relationship between structural elements and their function in the meiotic nucleus. In our initial experiments we will:

- Visualize and quantify the dynamic properties of meiotic chromosomes.

- Probe the role of chromosome architecture in controlling the distribution of crossovers.

- Investigate the essential role of the meiotic "pairing center" and associated proteins.

The project will integrate several technological approaches, including three-dimensional imaging, functional genomics, and genetics. We will use genetics to identify and characterize novel components of the chromosomeorganizational machinery that remodels the nucleus during meiosis. Using high-resolution fluorescence imaging, we will localize molecular components at the subcellular level to learn how they contribute to nuclear modeling. 


\section{Accomplishments}

The past year has seen significant expansion of this project in terms of personnel and experimental approaches.

The primary person working towards our first aim, biodynamic analysis of chromosome dynamics, is Peter Carlton, a postdoctoral fellow. His work has been expedited, and has enabled the construction of a biolistic device for worm transformation. He has begun a characterization and optimization of this device. In addition, he is developing expression vectors to He has also applied for individual follow-on funding from the NSF to support this aspect of the project.

We have identified a novel and exciting link between crossover regulation and chromosome architecture (Aim 2) that has formed the basis for two new projects in the lab. The key observation is that perturbation of chromosome synapsis on a single chromosome alters the pattern of crossovers on afl chromosomes in a way that indicates that meiotic regulation has been relaxed. We have preliminary evidence that this is due to the activation of a "synapsis checkpoint" that monitors the interactions between homologous chromosomes. Such a checkpoint has been proposed to exist in mammalian spermatogenesis. The nematode offers an excellent system to investigate this mechanism at a molecular level, which we are now pursuing. This project has been pursued through genetic and cytological experiments by a graduate student, Elsa Lee, and a rotation student, Alice Watson. Peter Carlton will analyze this phenomenon in terms of its effects on chromosome dynamics.

Our understanding of the role of the meiotic pairing center (Aim 3) has been refocused by a different recent observation in the lab: We now now that there is an apoptosis pathway in the germline that effectively culls nuclei with unrepaired double-strand breaks from the pool of potential gametes. This result has far-reaching implications for the genetic analysis of meiosis, since it indicates that the effects of any mutation that results in fuilures in chromosome synapsis und/or recombination may be only weakly reflected in the progeny produced by an affected animal - the most strongly-affected nuclei are essentially "invisible" to genetic analysis. To understand the direct effects of any mutation, we can introduce a mutation in a caspase that is required for the apopotosis pathway, and we are now characterizing a number of interesting mutants in the lab by comparing their effects with and without a functional germline apoptosis.

Moreover, this observation has provided new ways to identify lesions in the meiotic pairing center - while a pairing center deletion causes only a weak defect in an otherwise wild-type worm, in a caspase mutant worm it causes at very strong, dominant disruption of chromosome segregation. Thus, we are using this apoptosis-defective background as a way to screen for new Pairing Center mutations. This work is being carried out by a graduate student, Carolyn Phillips, who joined the lab in May, 2002.

Another new lab member is a postdoctoral fellow, Needhi Bhalla. Her project is focused on identification of proteins that interact with the meiotic Pairing Centers (part of Aim 3). To do this, she has initiated a genome-wide screen taking advantage of recently-developed RNA interference technology. This allows us to analyze specific gene products for potential meiotic roles using a library of bacteria that express double-stranded RNA corresponding to each $\mathrm{C}$. elegans gene. While this kind of screen requires more extensive set-up effort than conventional genetic approaches, it can lead to identification of functions that would not be seen in other ways and it vastly accelerates molecular analysis, since it bypasses the requirement to clone any gene product. Needhi has identified one potentially interesting candidate in her pilot screens, and will embark on a full-genome screen in early 2003 . In addition to this approach, she is planning to take advantage of the Lab's new capabilities in mass spectroscopic analysis of protein complexes to carry out a proteomic analysis of chromosome-associated factors in meiosis.

These latter two activities have now received some followon funding by an NIH RO1.

\section{Publications}

A.F. Dernburg, and G.H. Karpen, "A Chromosome RNAissance," Cell 111:159-162, October 18, 2002. http://www.cell.com/cgi/content/full/111/2/159/?highlight= dernburg

\section{Experimental and Computational Analysis of the Mechanisms and Logic of Eukaryotic Transcriptional Regulation}

\author{
Principal Investigators: Michael Eisen
}

Project No.: 00016

\section{Project Description}

The focus of our work is on characterization of the mechanisms and logic of transcriptional regulation in the yeast Stccharomyces cerevisiae, and, more recently, in the fruitfly Drosophila melanogaster. In yeust, we are particularly interested in understanding how variation in gene expression contributes to variation in phenotype, and 
in understanding how sequence variation in non-coding regions of the genome generate variation in gene expression. We employ a combitnation of experimental and computational approaches to address these questions. Our experimental work utilizes whole-genome deoxyribonucleic acid (DNA) microarrays to monitor the expression of every gene in the genome. We use these arrays to monitor gene expression changes following environmental perturbations (changes in temperature, nutrient availability and the concentration of toxic or potentially toxic metabolic byproducts such as ethanol). We are currently performing identical experiments in yeast strains with different genetic backgrounds (we work with a variety of laboratory strains and commercial and naturally isolated wine yeasts) and characterize differences in the environmental responses of the different strains.

The goal of these studies is to relate the observed differences in gene expression responses to differences in the cells' phenotypic response to the treatments. We hope both to understand how significant a role transcriptional regulation plays in phenotypic variation, and to understand the relationship between specific gene expression changes and specific phenotypic responses. In parallel, we are developing computational methods to explore how transcriptional information is encoded in the yeast genome sequence, to build predictive models that relate genome sequences to gene expression patterns, and to understand how variation in genome sequences will alter gene expression responses. Finally, we are beginning a project that combines phenotypic analysis, gene expression analysis and whole-genome genetic mapping techniques to directly link complex organismal phenotypes to gene expression and genome sequence.

We believe the method we are using will be a prototype for understanding the genetic basis of complex traits in more complex systems such as humans. In Drosophila, we are also interested in understanding how transcriptional regulatory information is encoded in (fully-sequenced) Drosophila genome, with a focus on the transcriptional regulatory cascades that choreograph the complex. developmental events that specify the form and function of the adult fly. Unlike in yeast, where transcriptional information for a given gene is contained in a relatively small piece of DNA immediately upstream of the gene, in animals like Drosophila, transcriptional information for a single gene is often spread over tens of thousands of basepairs. Therefore, the proximal chaltenge in analyzing the Drosophila (and also human and mouse) genomes is in identifying the regions of the genome that contain transcriptional information. We have begun to develop and apply novel computational methods to identify such regions.

\section{Accomplishments}

\section{Experimental Analysis of Gene Expression in Yeast}

We continue to routinely print and use whole-genome yeast microarrays consisting of 70 basepair synthetic oligonucleotides for each of the organism's 6,200 annotated genes. We have examined gene expression responses to changing temperature, starvation, diauxia (the shift from fermentable to non-fermentable carbon sources), anoxia, variable ethanol concentrations and exposure to copper sulfate in severat laboratory strains and natural isolates. Much of the response is conserved amongst these phenotypically distinct strains, but there are striking differences among the stains that can be related to their phenotypic differences. We are preparing two manuscripts describing this work for publication in the next year.

\section{Computational Analysis of Transcriptional Regulation in Yeast}

Organisms simplify the orchestration of gene expression by coregulating genes whose products function together in the cell. Many proteins serve different roles depending on the demands of the organism, and therefore the corresponding genes are often coexpressed with different groups of genes under different situations. This poses a challenge in analyzing whole-genome expression data, because many genes will be similarly expressed to multiple, distinct groups of genes. Because most commonly used analytical methods cannot appropriately represent these relationships, the connections between conditionally coregulated genes are often missed. We used a heuristically modified version of fuzzy k-means clustering to identify overlapping clusters of yeust genes based on published gene-expression data following the response of yeast cells to environmental changes. We have validated the method by identifying groups of functionally related and coregulated genes, and in the process we have uncovered new correlations between yeast genes and between the experimental conditions based on similarities in gene-expression patterns. To investigate the regulation of gene expression, we correlated the clusters with known transcription factor binding sites present in the genes' promoters. Our results give insights into the mechanism of the regulation of gene expression in yeast cells responding to environmental changes. Fuzzy k-means clustering is a useful analytical tool for extracting biological insights from gene-expression data. Our analyses suggests that a prevalent theme in the regulation of yeast gene expression is the condition-specific coregulation of overlapping sets of genes.

In collaboration with groups in the UC Berkeley Biostatistics, Statistics and Computer Science departments, we have developed an important new class of 
computational methods to infer the binding specificities of transcription factors from genome sequences. These methods integrate insights and data from structural biology into existing de novo binding site detection algorithms. We developed four distinct computational implementations of our general strategy and have demonstrated that they all significantly outperform existing methods under realistic conditions. Five manuscripts describing this work were recently submitted to Genome Biology.

\section{Computational Analysis of Transcriptional Information in the Drosophila Genome}

Just as coding genes in animals are organized in separate modular units (exons), transcriptional information in animals is also modularly organized. As stated in last year's report, we developed a computational method to identify cis-regulatory modules (CRMs) in the Drosophila genome based on the density of predicted binding sites for transcription factors that are known to act in a combinatorial manner. When we applied this method to find CRMs responsive to transcription factors active in the early Drosophila embryo, we were able to successfully recover 14 or 19 known CRMs, and identified at least 30 new binding site clusters that are potentially unrecognized CRMs. A strongly statistically significant number of these novel clusters are adjacent to genes that have patterned expression in the early embryo. We tested one of these novel clusters experimentally and confirmed that it is, indeed, a CRM that controls the expression of the adjacent gene. The methods were described in a paper in the Proceedings of the National Academy of Sciences (PNAS). Several additional predictions made in this paper have subsequently been confirmed by experiments performed by Sue Celniker's group in the Berkeley Drosophila Genome Project.

We have extended this work in several ways, developing more sophisticated methods for detecting clusters of binding sites based on hidden Markov models, and have performed extensive comparative analyses of the noncoding regions of the Drosophila melanogaster, Drosophila pseudoobscura and Anopheles gambiae genomes. A National Institutes of Health (NIH) grant submitted based on this work received a very high priority score and is almost certain to be funded starting April 2003.
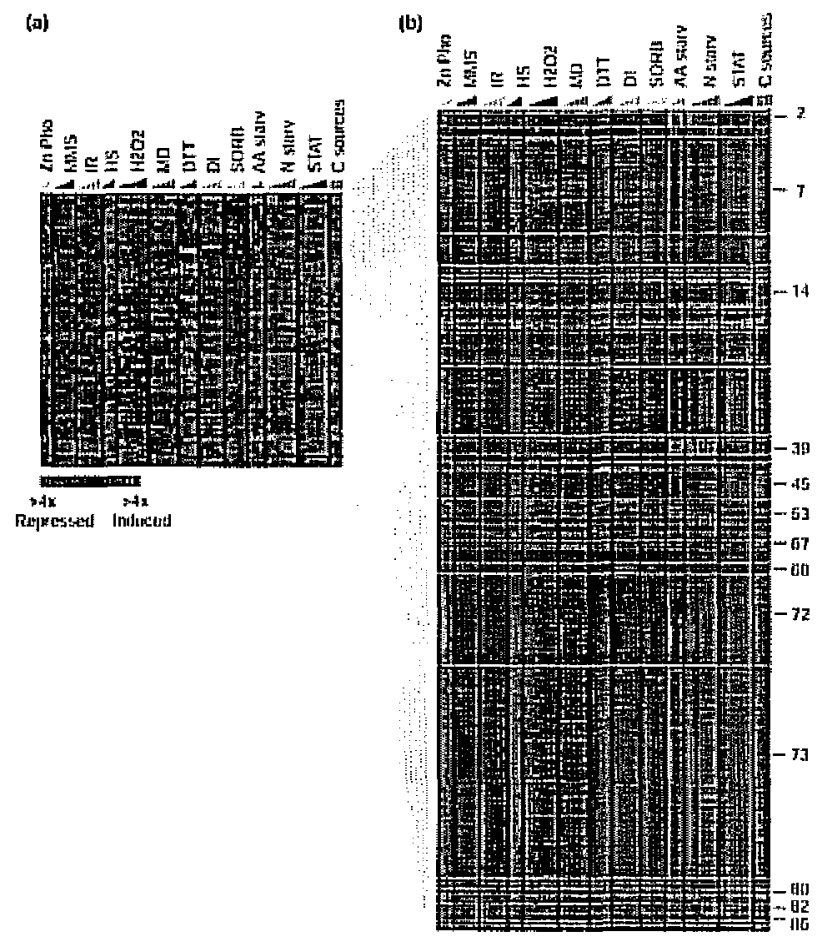

Figure 2: (a) Each row in this diagram represents one of the 91 centroids identified by fizzy $k$-means clustering. The data representation is the same as described in Figure 2 (b). Genes were assigned to each of the centroids shown in (a) using a membership cutoff of 0.08 , as described in the text. Each cluster of selected genes is separated by a horizontal gray line. Examples of functionally related clusters of gentes are indicated by numbers to the right of (b): cluster 2, amino-acid biosynthesis genes; cluster 7 . genes induced as part of the environmental stress response; cluster 14, mitochondrial protein synthesis genes; cluster 39. genes involved in nitrogen utilization; cluster 45, oxidative phosphorylation and respiration components; cluster 53, specific amino-acid transporters; cluster 67, glycolysis genes; cluster 68, proteasome components; cluster 72, secretion, protein synthesis, and membrane synthesis genes; cluster 73 , genes repressed as part of the environmental stress response; cluster 80, aminoacid biosynthesis genes; cluster 82, G2/M cyclins; cluster 86 , histone genes. 


\section{Publications}

B.P. Berman, Y. Nibu, B. Pfeiffer, P. Tomancak, S. Celniker, M.S. Levine, G.M. Rubin, and M.B. Eisen, "Exploiting Transcription Factor Binding Site Clustering to Identify CIS-regulatory Modules Involved in Pattern Formation in the Drosophila Genome," PNAS, vol. 99, no. 2. pp. 757-762, January 22, 2002.

http://rana.lbl.gov/papers/Berman_PNAS_2002.pdf

S. Keles, M. van der Laan, and M.B. Eisen, "Identification of Regulatory Elements Using a Feature Selection

Algorithm," Bioinformatics, vol. 18, no. 9, pp. 1167-1175, September 2002.

http://rana.1bl.gov/papers/Keles_Bioinformatics_2002.pdf

A.P. Gasch and M,B. Eisen, "Exploring the Conditional Coregulation of Yeust Gene Expression through Fuzzy Kmeans Clustering," Genome Biology, 3(11):research0059.I0059.22 , October 10, 2002.

http://rana.Ibl.gov/papers/Gasch_GB_2002.pdf

D.Y. Chiang, A. Moses, M. Kamvysselis, E.S. Lander, and M.B. Eisen, "Discovery of Conserved Sequence Pair Templates Associated with Gene Expression Differences in Yeast," Seventh Annual hiternational Conference on Research in Computtational Molecular Biology (RECOMB 2003), April 2003, accepted for publication.

M.B. Eisen, "All Motifs Are Not Crented Equal: Siructural Properties of Transcription Factor - DNA Interactions and the Inference of Sequence Specificity," Genome Biology, submitted for publication.

K. Kechris, E. van Zwet, P. Bickel, and M.B. Eisen, "Detecting DNA Regulatory Motifs by Incorporating Positional Trends in Information Content," Genome Biology, submitted for publication.

E. van Zwet, K. Kechris, P. Bickel, and M.B. Eisen, "Estimating Motifs Under Order Restriction," Genome Biology, submitted for publication.

S. Keles, M. van der Laan, B. Xing, and M.B. Eisen, "Detecting Regulatory Motifs with Entropy Constraints in DNA Sequences, Genome Biology, submitted for publication.

D.Y. Chiang, A. Moses, M. Kamvysselis, and E.S. Lander, M.B. Eisen, "Discovery of Conserved Sequence Pair Templates Associated with Gene Expression Differences in Yeast, Genome Biology, submitted for publication.

\section{Structural Studies of Presenilin-1, a Membrane Protein Critical to the Onset of Alzheimer's Disease}

Principal Investigators: Bing Jap

Project No.: 02030

\section{Project Description}

Presenilin- 1 is a major component of $\gamma$-secretase, which is believed to play a crucial role in the onset of Alzheimer's clisease. Presenilin-1 and its homologue, presenilin-2, are the catalytic components of $\gamma$-secretase, which cleave the C-terminal end of amyloid precursor protein (APP). This processing of APP together with its cleavage at the Nterminal end by $\tilde{\beta}$-secretase produces amyloid $\beta$ fragments (AB) that are associated with the formation of amyloid plaques in the brain of Alzheimer's patients. Presenilin-1 is a novel aspartyl protease having its catalytic active site embedded in the membrane. Mutations of this membrane protein and/or its homologue presenilin-2 have been associated with the cause of an aggressive form of Alzheimer's disease and are potential targets for therapeutic drugs.

The aim of this project is to obtain sufficient quantities of purified presenilin-1 in an effort to produce crystals for subsequent $x$-ray crystallography structure determination of this membrane protein. This involves the cloning of the gene, establishing a suitable overexpression system, purification and crystallization trials. When suitable crystals are produced, efforts outside the scope of this project will be needed to determine the molecular structure of presenilin-1. The structure of this membrane protein should provide us with atomic level details of this novel enzyme, yjeld insights into the mechanism of its proteolytic activity within the membrane bilayer and help in the design of therapeutic drugs.

\section{Accomplishments}

We have cloned the presenilin-1 gene from a human brain c-DNA library. Attempts at the overexpression of the gene using $E$. coli expression systems have produced insignificant levels of expression. This is not unusual for the overexpression of human proteins, especially human membrane proteins.

We have also cloned presenilin- 1 in a yeast expression system where it is currently expressing at a relatively low 
level, but significantly higher than was previously

expressed in E. coli. As this expression level is significantly less than ideal for large scale purification purposes, we will also continue to investigate alternative expression systems including other yeast strains and mammalian cell lines.

Recently, it has been determined that the active $\gamma$-secretase is actually a complex of at least three additional membrane proteins. One of these proteins, nicastrin, has been shown to play a role in the regulation of substrate proteolysis. Nicastrin contains a single transmembrane region with a large extra-membrane domain. We have successfully cloned this membrane protein for overexpression in $E$. coli. To improve the probability of success in obtaining suituble crystals, we are also preparing to construct a nicastrin clone in which the transmembrane domain has been removed.

\section{Tracking Proteins in Cells Using Light and Soft X-ray Microscopy}

Principal Investigators: Carolyn Larabell

Project No:: 01025

\section{Project Description}

$\mathrm{X}$-ray microscopy can be used to localize proteins in whole, hydrated cells at 5 to 8 times better resolution than possible with light microscopy. Until recently, however, the information was presented as a two-dimensional projection of three-dimensional data. We are currently developing the technology to do tomographic reconstructions of whole cells using $\mathrm{x}$-raty tomography. We will use light microscopy to examine the dynamics of specific proteins that have been tagged with the fluorescent marker called green fluorescent protein (GFP) in live cells. After the dynamics of the protein have been determined, the cells will be fixed, labeled with commercially available antibodies to GFP, and then examined in the $\mathrm{x}$-ray microscope to determine the subcellular location of the protein. The use of the $x$-ray cryo-tomography will generate unique, high-resolution three-dimensional information about the subcellular localization of the protein throughout the entire cell. The development of this methodology will provide a highthroughput method for structure-function analyses of proteins.

Our initial studies will be designed to demonstrate the feasibility of these studies. We will transfect human mammary epithelial cells with GFP constructs for proteins with characteristic labeling patterns. The dynamics of the fluorescent proteins will be examined by monitoring the living cells using confocal or multi-photon microscopy. Optical sections of the cells will be collected over time and three-dimensional reconstructions of the labeled proteins within the cell will be obtained. After the live cell studies are completed, the cells will be fixed and labeled using commercially available anti-GFP antibodies followed by immunogold labeling for the $x$-ray microscope. A series of images at 150 different angles will be collected for tomographic reconstruction.

\section{Accomplishments}

We have tracked numerous GFP-tagged proteins using both standard confocal and multi-photon microscopy in thin and thick specimens. The resolution achieved with light microscopy is approximately 200 nanometers. In many cases, we need better resolution to determine the precise location of the proteins. We previously used soft $\mathrm{x}$-ray microscopy to obtain this information and achieved better than 50 nanometer resolution. Because the images obtained were 2-D projections of 3-D data, it was obvious that we would have to obtain tomographic reconstructions to accurately determine the location of those proteins. Therefore, we have been developing the capacity to obtain 3-D reconstructions of whole, hydrated cells.

We used $x-$ ray cryo-tomography to obtain 3-D reconstructions of whole, rapidly frozen yeast cells. The yeast we examined, Saccharomyces cerevisiae, is an important model for understanding cellular and molecular processes in enkaryotes. It was the first eukaryote sequenced and many of its protein-encoding genes (approximately $30 \%$ ) have mammalian homologs. In addition, almost $50 \%$ of the human genes implicated in heritable diseases in humans have a homologue in yeust. Consequently, this is an important model organism for modern proteomic analyses. We are developing the ability to obtain tomographic reconstructions of immunolabeled yeast to facilitate analyses of the three-dimensional distribution of specific proteins involved in signaling pathways.

Live yeast at varying stages of the cell cycle were positioned in a $10 \mu \mathrm{m}$ diameter glass capillary to facilitate collection of unobstructed images of the yeast. A series of yeast were first examined in the light microscope then rapidly frozen and placed in the $x$-ray microscope where they were maintained at cryogenic temperatures. For each reconstruction, 45 full-field projection images were collected at $4^{\circ}$ intervals through $180^{\circ}$ of rotation. All 45 images were aligned to a common axis of rotation using fiducial markers (60 nanometer diameter gold particles added to the capillary) and the program "Spider". These tilt series were then viewed as individual projection images as well as played back as a movie. A single projection image 
of an intact yeast, which is in the early budding stage, and portions of several nearby yeast can be seen in Figure 1A. Numerous overlapping vesicles and organelles can be seen within the yeast (small white circles) but it is difficult to distinguish the precise boundaries since this is a projection of all structures located in the five-nicron thick yeast. Two of the 60 nanometer diameter gold particles are easily discerned, demonstrating the superb resolving power of $x$ ray microscopy. Since each projection image contains information about all of the structures in the 5-micron diameter yeast compressed into one imige, interpretation of the data requires tomographic reconstruction. The tomographic reconstructions were calculated using a weighted back-projection aIgorithm, and one of the sections from a reconstruction is shown in Figure 1B. The individual organelles and vacuoles are now distinctly resolved. Since the organic material in the yeast absorbs approximately an order of magnitude more than the surrounding water, these structures are visualized based on their $\mathrm{x}$-ray absorption coefficient; consequently it is not necessary to use contrast enhancement reagents. Furthermore, the information obtained is quantifiable. Once the data has been obtained, computer algorithms can be used to selectively reveal the internal structures.

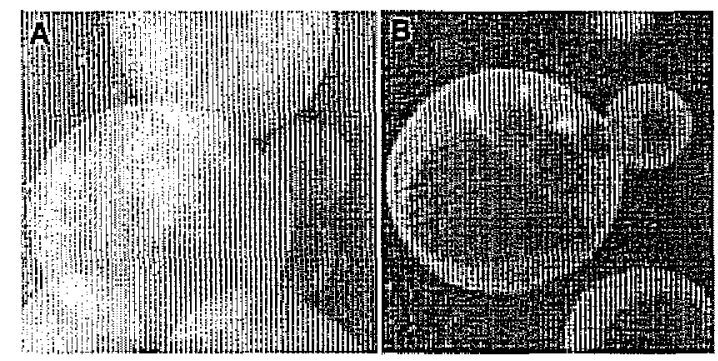

Figure 3: X-ray cryo-tomography of rapidly frozen, intact yeast, Saccharomyces cerevisiae. $(A)$ shows a single projection inage of the five-micron diameter yeast. (B) shows a single section of the computer reconstructed data. The densest structures (lipid droplets) appear white.

\section{Publications}

W. Meyer-Ilse, D. Hamamoto, A. Nair, S.A. Lelievre, G. Denbeaux, L. Johnson, A.L. Pearson, D. Yager, M.A. LeGros, and C.A. Larabell, "High Resolution Protein Localization Using Soft X-ray Microscopy," J. Micros. 201, 395-403, 2001.

\section{Systems Biology: Biological Input- output Devices}

Principal Investigators: Saira Mian

Project No.: 02031

\section{Project Description}

Biological systems surpass current silicon-based technologies in areas such as sensor fusion, pattern recognition, adaptability and information storage, retrieval, processing and related tasks. Irrespective of the level of resolution at which it is viewed, a molecular, (sub)cellular, multicellular or higher level system transforms internal and external inputs to yield outputs that maintain homeostasis or ensure a smooth transition to a new state. The common feature is large numbers of potentially unreliable parts interconnected in unknown, irregular and time-varying ways cooperating to generate a coherent and unified response.

Biological systems are in vivo input-output devices with the capacity to perform parallel and distributed computing in a robust, reliable, secure, error- and fault-tolerant manner. Enhanced knowledge of the means by which this complex task is solved provides an opportunity to control existing devices, fashion novel devices for designed purposes and create fundamentally new information systems and technologies.

The aims of this project are the development and application of mathematical, statistical and computational methods for investigating input-output devices. The two complementary approaches being employed to realize these goals are data-driven modeling and conceptual modeling.

\section{Accomplishments}

Biologists and clinicians are adopting high-throughput genomics, proteomics and related technologies to assist in interrogating normal and perturbed systems such as unaffected and tumor tissues. In cancer biology for example, molecular profiling studies of different types of specimens are motivated largely by a desire to create clinical decision support systems for accurate cancer classification and to identify robust and reliable targets for intervention, diagnosis and imaging ("biomarkers"). These specific biological tasks can be equated with the general 
statistical problems of classification and prediction, and relevant feature identification.

The past year has focused on (1) sparse solutions to highdimensional classification and regression problems and (2) robust optimization and linear programming as techniques for solving these statistical tasks. Although the associated theory and attendant algorithms have been studied in engineering and related disciplines, they have not been exploited in computational biology. When applied to a variety of real world genomic and proteomic data sets, the results demonstrate the potential and utility of such ideas.

For example, nominal sparse hyperplanes were proposed as statistical algorithms for classifiying samples or data points into one of two categories (classification and prediction) and defining a small subset of discriminatory features (relevant feature identiftcation). Formulating the underlying problem as a linear program allowed widely available, open source software packages to be used. Unanticipated biological insights and novel specific predictions of interest to experimentalists and physicians were generated when sparse hyperplanes were employed for retrospective studies of molecular profiling data.

\section{Publications}

L.R. Grate, C. Bhattacharyya, M.I. Jordan, and I.S. Mian, "Integrated Analysis of Profiling and Sequence Data," Mechantisms of Ageing d Development, in press.

L.R. Grate, C. Bhattacharyya, M.I. Jordan, and I.S. Mian, "Simultaneous Relevant Feature Identification and Classification in High-dimensional Spaces," in Proceedings, Workshop on Algorithms in Bioinformatics, September 2002, Rome, Italy.

C. Bhattacharyya, L.R. Grate, A. Rizki, D.C. Radisky, F.J. Molina, M.I. Jordan, M.J. Bissell, and I.S. Mian, "Simultaneous Relevant Feature Identification and Classification in High-dimensional Spaces: Application to Molecular Profiling Data," Signal Processing, in press.

S. Singh, O. Steinberg-Neifach, I.S. Mian, and N.F. Lue, "Analysis of Catalytic and Non-catalytic Sunbits of Telomerase in Candida Albicans: A Direct Role for Est $1 p$ in Telomere Extension," Eukaryotic Cell, 1, pp. 967-977 (2002). 



\section{Materials Sciences Division}

\section{Inorganic Liquid Crystals}

Principal Investigators: A. Paul Alivisatos, Peidong

Yang and Jean Frechet

Project No.: 02032

\section{Project Description}

The goal of this project is to develop a novel class of liquid crystals based upon inorganic nanorods of semiconducting, metallic, and magnetic systems. Liquid crystals of organic molecules and macromolecules are widely studied for their theoretical interest, as well as for their practical utility in a wide range of technologies, including flat panel displays. We propose to take advantage of recent developments in the control over the sites and shapes of inorganic nanocrystals to prepare a new class of liquid crystals.

To accomplish this, inorganic nanocrystals of $\mathrm{CdSe}, \mathrm{Au}$, $\mathrm{Co}$, and $\mathrm{BaCro}_{4}$ will be produced by colloidal wet chemical techniques. The shapes of the nanocrystals will be precisely controlled by employing binary mixtures of organic surfactants to modulate the growth rates of different crystallographic directions. The nanorods will be examined by a wide range of analytical techniques, including $x$-ray diffraction (XRD), transmission electron microscopy (TEM), (x-ray photoelectron spectroscopy) XPS, and atomic force microscopy (AFM). Nanorod liquid crystal phases will be investigated by examining optical birefringence from dense solutions of nanorods, as well as by small angle $\mathrm{x}$-ray scattering. Once liquid crystal phases are obtained, we will attempt to order them using external electric and magnetic fields.

\section{Accomplishments}

During this year we successfully investigated the liquid crystalline phases of inorganic nanocrystals. Our first report appeared as a cover article in Nano Letters In that report we used polarized microscopy to observe the formation of liquid crystalline phases of CdSe nanorods at high density in cyclohexane. Since the initial report, we have made significant progress as follows:

- We examined the response of CdSe nanorods to external electric fields. In this study we showed that the nanorods have a large permanent electric dipole moment, in the neighborhood of 200 Debye for a $3 \mathrm{~nm} \times 60 \mathrm{~nm}$ rod. The knowledge of dipoledipole induced interactions between rods is essential for future study of the liquid crystals.

- We investigated the deposition of liquid crystal layers onto substrates by TEM and we showed that complex patterns can be arranged and controlled.

- We investigated the nanorod liquid crystals by small angle $x$-ray scattering and by nuclear magnetic resonance (NMR), in order to determine phase diagrams.

- We have initiated a study of the liquid crystalline phases of disk-shaped iron oxide nanocrystals.

All four of these items ate in the stage of being written up for publication.

\section{Publications}

L. Li, L. Manna, J. Hu and A.P. Alivisatos, "Semiconductor Nanorod Liquid Crystals," Nano Letters, 2, No. 6, 557-560, June 2002.

L. Li., et al., "Origin and Scaling of the Permanent Dipole Moment in CdSe Nanorods," Phys. Rev. Let., January 2003, in press.

L. Li, et al., "Semiconductor Nanorod Liquid Crystals and their Assembly on a Substrate," Advanced Materials, December 2002, in press.

\section{Functional Bone-like Materials: a Biomimetic Synthetic Approach}

Principal Investigators: Carolyn Bertozzi

Project No.: 01026

\section{Project Description}

The goal of the proposal is to develop new materials that are tough yet lightweight and fracture resistant. Such materials would find widespread use in industry. Nature creates a material-bone-that embodies these properties and our goal is to design artificial materials using natural bone as a guide. Bone consists of a microcrystalline inorganic composite called hydroxyapatite, embedded in an 
organic matrix that provides flexibility and elasticity. This paradigm will be recreated using synthetic constructs. Organic materials will be designed for micronucleation of inorganic crystals and the composites analyzed with respect to physical properties.

Organic microcrystalline materials will be generated from polymerized diacetylene arrays generated by self-assembly. Polydiacetylenes form various organized structures on the micron scale, such as tubes, liposomes, and ribbons, and these organized structures will be used to promote the crystallization of hydroxyapatite $\left[\mathrm{Ca}_{10}\left(\mathrm{PO}_{4}\right)_{6}(\mathrm{OH})_{2}\right]$, the ceramic found in natural bone. The microcrystalline nature of the organic scaffold will dictate the organization of inorganic crystals in the bulk material. The resulting composites will be further incorporated into a hydrogel polymer scaffold to provide physical shape and elasticity. Physical measurements of toughness and fracture resistance will be performed.

\section{Accomplishments}

Bone consists of microcrystalline calcium apatites and collagen, an elastic porous protein matrix that is decorated with mineral-nucleating phosphoproteins. Our rational design of artificial bone-like material uses natural bone as a guide.

Hydrogel polymers that possess anionic groups suitably positioned for nucleating biominerals, and therefore mimic the natural function of the collagen-phosphoprotein matrix in bone, were designed to direct template-driven biomineralization in a microscopically controlled fashion. Different mineralization techniques were developed to enable the formation of 3-dimensional hydrogel-calcium phosphate composites with either porous microstructures suitable for potential tissue penetration or excellent polymer-mineral interfacial aftinity (Figure A) that is desirable for bone mimics with enhanced biocompatibility and interfacial mechanical properties. Chemical, structural and mechanical properties of these novel hydrogels and composite materials were characterized by contact angle measurements, fourier transform infrared spectroscopy (FTIR), scanning electron microscope energy dispersion spectroscopy (SEM-EDS), X-ray diffraction (XRD) and micto-indentation analyses.

In-vitro cell cultures have shown that both osteosarcoma TE85 and normal human osteoblasts (NHOst) were able to attach, spread and proliferate on these bonelike composites. The expressions of important osteoblastic marker proteins upon the attachment to these materials were examined.

To further control the order and conformation of the mineral nucleating ligands display on a nanoscopic level, a library of self-assembling bolaamphiphilic lipids functionalized with anionic surface residues were designed and synthesized. We have shown that these lipids can selfassemble and polymerize into highly crystalline nanoribbons and sheets under ultraviolet (UV) irradiation, and are capable of templating the growth of crystalline apatite crystals when exposed to various mineralization solutions.

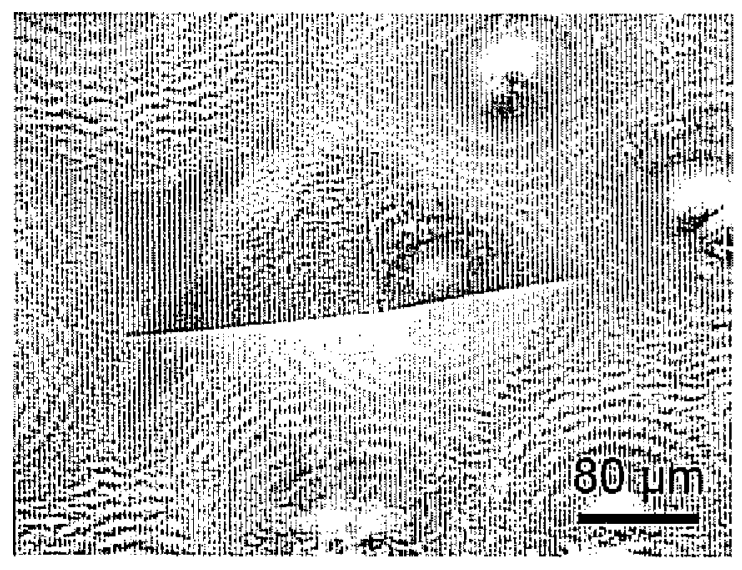

Figure 1: SEM showing an indent formed on the surface of a pHEMA-calcium phosphate (CP) composite using a Vickers microindenter with a load of $5 \mathrm{~N}$. The CP layer did not delaminate, indicating excellent interfacial adhesion between the polymer substrate and the mineral layer.

\section{Publications}

J. Song, E. Saiz and C. R. Bertozzi, "A New Approach to Mineralization of Biocompatible Hydrogel Scaffolds: An Efficient Process Towards 3-Dimensional Bonelike Composites," Journal of the American Chemical Society, 2002, in press.

J. Song, E. Saiz and C. R. Bertozzi, "Preparation of pHEMA-CP Composites With High Interfacial Adhesion via Template-Driven Mineralization," Joturnal of the European Ceramic Society, Submitted for publication, 2002

J. Song, E. Saiz, A. P. Tomsia and C. R. Bertozzi, "Design, Synthesis and In Vitro Evaluation of Biomimetic Bonelike Composites and Novel Bioactive Glass Coatings," Materials Science Forum, Trans Tech, Switzerland, In preparation, 2003

J. Song and C. R. Bertozzi, "Functional Bone-like Materials via Biomimetic Syntheses, Society for Biomaterials Workshop: Nanobiomaterials," Invited talk, April 24, 2002

J. Song, E. Saiz and C. R. Bertozzi, "Biomimetic hydrogel - Hydroxyapatite Composites as Artificial Bone-like Materials, International Workshop on Ceramic and Metal 
Interfaces: Control at the Atomic Level," Invited talk, June $23-27,2002$,

J. Song and C. R. Bertozzi, "Bio-inspired Functional SelfAssembling Conjugated Polymers," MRS 2003 Spring Meeting, Abstract submitted, April 21-25, 2003

\section{Condensation of Indirect Excitons in Coupled Quantum Well Nanostructures}

Principal Investigators: Daniel Chemla and Leonid

Project No.: 01027

\section{Project Description}

A system of spatially indirect (interwell) excitons in coupled quantum wells (CQWs) provides a unique opportunity for experimental study of two-dimensional (2D) light bosons thermalized down to ultralow temperatures. The low-temperature, high-density excitonic matter is a potential candidate for observing Bose-Einstein condensation. Recent experimental studies of indirect excitons in CQWs have revealed nonclassical signature: seen in exciton transport and photoluminescence that are not explained within single exciton models and indicate possible coherency in the exciton system. In this project, we propose novel coherent spectroscopy experiments that could reveal macroscopic quantum effects in the system of indirect excitons and, in particular, could identify the exciton condensation using new CQW samples specially designed for this project and novel experimental techniques with increased sensitivity to quantum coherence.

The coherence in an exciton system in CQW heterostructure can be directly detected by the coherence of the light it emits. We have prepared new AlAs/GaAs and GaAs/A1GaAs samples especially designed and optimized for this project. We propose to study the second and the fourth order correlation functions of the light emitted by these samples through: (1) (a) the speckles of Rayleigh scattering and (b) the study of the photon statistics by the measurement of the correlation of intensities registered by two different detectors; (2) the search for the excitonic insulator state by a time -resolved photoluminescence experiment; and (3) to realize experiments for exciton condensate in the traps, similar to the recent experiments for the atom Bose-Einstein condensate.

\section{Accomplishments}

This last year, we demonstrated that the magnetoexciton effective mass is determined by the coupling between the center-of-mass motion and internal structure and becomes overwhelmingly larger than the surn of the electron and hole masses in high magnetic fields.

We also calculated the internal structures and dispersion relations of spatially direct and indirect excitons in single and coupled quantum wells in a magnetic field perpendicular to the well plane. We found a sharp transition from a hydrogenlike exciton to a magnetoexciton with increasing the center-of-mass momentum at fixed weak field. At that transition the mean electron-hole separation increases sharply and becomes $P / B$, where $P$ is the magnetoexciton center-of-mass momentum and $B$ is the magnetic field perpendicular to the quantum well plane. The transition resembles a first-order phase transition. The mignetic-field-exciton momentum phase diagram describing the transition was constructed. The calculated dispersion relations and effective masses are in agreement with the experimental data. We discussed the impact of magnetic field and sample geometry on the condition for observing exciton condensation.

Finally, we observed condensation of indirect excitons at the bottom of potential traps, similar to that of atom BEC. In our experiments the traps were formed by fluctuations of the in-plane random potential. The exciton condensation was directly verified by the spatial shrinkage of the exciton cloud near the bottom of the potential trap as the temperature is reduced.

\section{Publications}

Yu. E. Lozovik, I. V. Ovchinnikov, S. Yu. Volkov, L. V. Butov, and D. S. Chemla, "Quasi-two-dimensional Excitons in Finite Magnetic Fields," Physical Review B 65, 235304. 2002.

L.V. Butov, C.W. Lai, A. L. Ivanov, A. C. Gossard, and D. S. Chemla, "Towards Bose-Einstein Condensation of Excitons in Potential Traps, Nature 417, 47, 2002. 


\section{Nanoscale Electronic Phase Separation: A New Paradigm for Complex Electronic Materials}

Principal Investigators: J. C. Séamus Davis, Dung-

Hai Lee, and Joseph Orenstein

Project No.: 02033

\section{Accomplishments}

The two major accomplishment of the first year of this project were:

- discovery of the remarkable and unexpected extent of inhomogeneity in a class of high- $T_{t}$ superconductors.

- exploration of its consequences for quasiparticle scattering, electrical conductivity, and the superconducting transition temperature, $\mathrm{T}^{*}$.

The extent of inhomogeneity in the bismuth-based cuprate superconductor $\left(\mathrm{Bi}_{2} \mathrm{Sr}_{2} \mathrm{CaCl}_{2} \mathrm{O}_{8+6}\right.$ (BSCCO) was revealed through scanning tunneling spectroscopy. Spectroscopy with atomic scale resolution was performed in an ultra-low vibration environment on BSCCO surfaces cleaved at low termperature and in high vacuum. The stability of the system permitted mapping the local density of states (LDOS) with atomic resolution and position registry throughout a large area (several hundred Angstroms on a side). The LDOS maps revealed enormous variation in both the amplitude and shape of the superconducting gap as a function of position on the sample surface. The gap magnitude varies by $50 \%$ with a change in position of only a few tens of Angstroms. This extent of spatial inhomogeneity was entirely unanticipated, despite extensive characterization of these materials with other spectroscopic techniques.

Superficially, the patterns of inhomogeneity appear to be random in nature. However, motivated by theoretical work by members of our team, the LDOS maps were investigated by Fourier transform analysis. Fourier transforms of the LDOS maps are shown in the accompanying figure 2 for several values of the quasiparticle energy. The presence of well defined spots in several of the momentum space images (those in the range from -12 to $-20 \mathrm{meV}$ are particularly striking) show that variation of LDOS at each energy results from the superposition of ripples with welldefined wavelength and orientation in real space. Further analysis shows that the wavevector of the ripples changes (or disperses) systematically with energy. Again relying on theoretical insights developed in this project, the shape of the Fermi contour and the variation of the gap energy along it were determined from the dispersion relations. Finally, the observation of inhomogeneity in the density of states suggests a similar inhomogeneity in the local superfluid density. Our group showed, both theoretically and experimentally, that such iinhomogeneity explains longstanding mysteries concerning the ac electrical conductivity of many cuprate superconductors. 


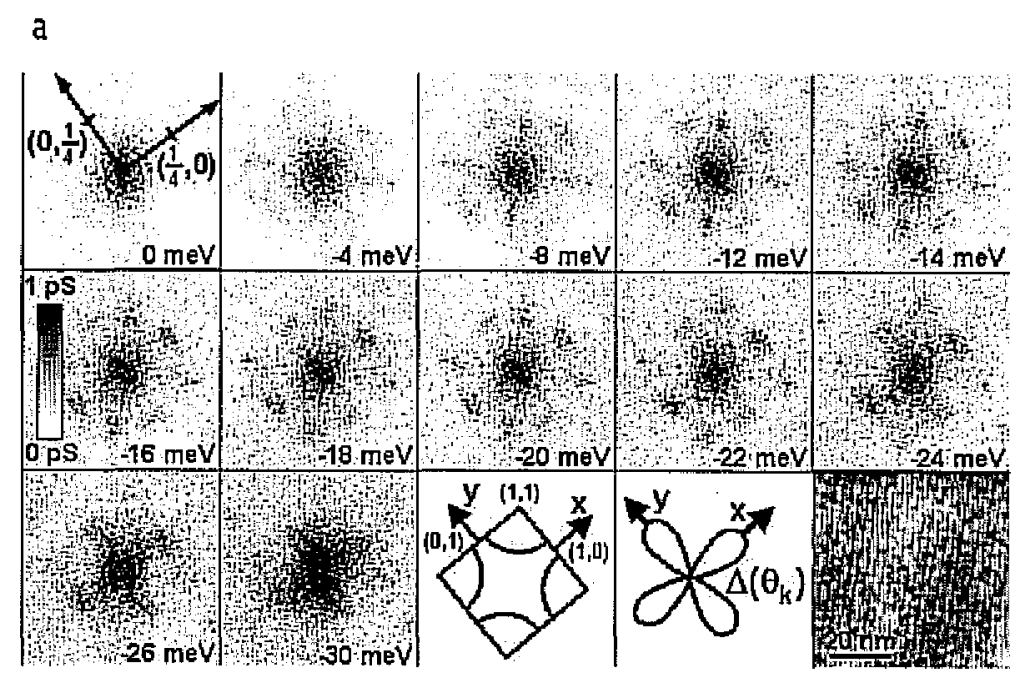

Figure 2: Images of quasiparticle interference in an inhomogeneons high-T $T_{r}$ stperconductor. Each frame depicts the spatial Fourier transform of the local density of states (LDOS) of quasiparticles at the energy indicated. The two illustrations show the supercondicting gap as a function of direction in momentum space (right panel) and the Fermi contour of the normal state (left panel).

\section{Publications}

K.M. Lang, V. Madhavan, J.E. Hoffman, E.W. Hudson, H. Elsaki, S. Uchida, J.C. Davis, "Imaging the Granular Structure of High- $T_{\mathrm{c}}$ Superconductivity in Underdoped $\mathrm{Bi}_{2} \mathrm{Sr}_{2} \mathrm{CuCu}_{2} \mathrm{O}_{8+5}$ " Nature, 415, 412 (2002).

J.E. Hoffman, K McElroy, D.-H. Lee, K.M. Lang, H. Eisaki S. Uchida, and J.C. Davis, "Imaging Quasiparticle Quantum Interference in $\mathrm{Bi}_{2} \mathrm{Sr}_{2} \mathrm{CaCu}_{2} \mathrm{O}_{8+8}$, Science 297, 1148 (2002).

K. McElroy, R.W. Simmonds, J.E. Hoffmän, D.-H. Lee, J. Orenstein, H. Eisaki, S. Uchida, and J.C. Davis, "Determining the Momentum-space Structure of Cuprate Quasiparticles from Quantum Interference Images," Nattire, accepted for publication.

J. Orenstein, "Optical Conductivity of a Superfluid Density Wave," Physca $C$, accepted for publication.

N. Gedik, J. Orenstein, R. Liang, D.A. Bonn, W.N. Hardy, "Propagation of Nonequilibrium Quasiparticles in a High$\mathrm{T}_{\mathfrak{t}}$ superconductor," Science, submitted for publication.

N. Gedik, J. Orenstein, R. Liang, D.A. Bonn, W.N. Hardy, "Dynamics of nonequilibrium quasiparticles in $\mathrm{YBa}_{2} \mathrm{Cu}_{3} \mathrm{O}_{6.5,}, "$ in preparation.

Steven A. Kivelson, Dung-Hai Lee, Eduardo Fradkin, Vadim Oganesyan., "Competing Order in the Mixed State of High Temperature Superconductors'," Phys Rev. B 66, 144516 (2002).

D.-H. Lee, Duality between Unidirectional Charge-DensityWave Order and Superconductivity'," Phys. Rev. Lett. 88, 227003 (2002).

Q-H Wang, J. H. Han and D-H Lee, "Pairing Near the Mott Insulating Limit," Pltys. Rev. B 65, 054501 (2002).

\section{Impurity Overlayers in Semiconductor Epitaxy: Expanding the Limits of Alloy Composition and Nanostructure Synthesis}

Principal Investigators: Oscar Dubón, Jr.

Project No.: 01028

\section{Project Description}

The presence of a surface layer of segregating impurities during epitaxial growth can produce dramatic changes in the morphology of the resulting film. The impurity overlayer, or surfactant, changes the arrangement of substrate adatoms and the mobility of depositing atoms and 
therefore represents an experimental parameter that can be exploited to influence growth kinetics. Control over growth kinetics is an essential ingredient for the effective manipulation of self-organizing processes that lead to the formation of periodic semiconductor nanostructures including island arrays and alloy superlattices.

The surfactant typically consists of only one or a fraction of one atomic layer of impurities. However, at greater thickness the impurity overlayer itself behaves differently due to strong intralayer interactions and rapidly assumes bulk-like behavior. "This change in overalyer properties provides additional opportunities to control film morphology. In this project, we have undertaken a study of impurity overlayers in the two thickness regimes and their influence on the growth processes.

A layer of lead atoms is used in the molecular beam epitaxial growth of group-IV-based alloys to probe surfactant effects on spontaneous pattern formation. The choice of $\mathrm{Pb}$ as a surfactant is based on recent experiments showing that a $\mathrm{Pb}$ layer in $\mathrm{Ge}$ and $\mathrm{Si}$ thin-film epitaxy dramatically reduces the minimum temperature for epitaxial growth, and, unlike other commonly used surfactant species, $\mathrm{Pb}$ does not degrade the electrical properties of the films. Alloys highly enriched with the element Sn are the primary focus of this study. These materials provide novel possibilities for the investigation of alloy compositional modulation in highly mismatched systems.

It is known that gold behaves in a surfactant-like manner in silicon and germanium homoepitaxy when it is on the order of one atomic layer in thickness. However, for gold layers nanometers in thickness (and greater), heating to temperatures above $360^{\circ} \mathrm{C}$ leads to a molten solution of $\mathrm{AL}$ with substrate atoms of $\mathrm{Ge}$ or $\mathrm{Si}$ dissolved in it. The melted layer breaks up into small droplets which then catalyze growth by supersaturation of the droplet with the vapor flux of the depositing species in a so-called vapor-liquid-solid process. This approach has been widely used for the synthesis of semiconductor nanowires. We are investigating the use of gold layers to synthesize germanium island arrays.

\section{Accomplishments}

To date we have deposited a variety of thin-films. In addition to homoepixial films of $\mathrm{Si}$ and $\mathrm{Ge}$, SiGeSn alloys on Ge substrates have been grown. For growth at a substrate temperature of $300^{\circ} \mathrm{C}$ and growth rates on the order of $1 \mathrm{As}^{-1}$, up to 1.1 at $\%$ of $\mathrm{Sn}$ may be incorporated into the film. The Sn distribution across the thickness of the film is uniform. Tin in excess of this amount segregates to the surface during growth and forms islands. Growth at 100 ${ }^{\circ} \mathrm{C}$ showed a significant reduction in Sn segregation with a rapid decay in crystalline quality with increasing film thickness. Initial experiments using $\mathrm{Pb}$ as a surfactant have been performed. It is expected that the $\mathrm{Pb}$ overlayer will promote epitaxy at the lower growth temperatures needed to suppress segregation. While it appears that the presence of $\mathrm{Pb}$ leads to improved epitaxy, further experiments are necessary to clearly determine the effect of the $\mathrm{Pb}$ overlayers on film morphology.

Using gold layers up to $3 \mathrm{~nm}$ in thickness, we have grown germanium islands on $\mathrm{Ge}(111)$ at a substrate temperature of $700^{\circ} \mathrm{C}$. The islands appear to grow epitaxially by the vapor-liquid-solid (VLS) process, retaining the symmetry of the substrate. In general, each $\mathrm{Ge}$ island is decorated by a solidified gold droplet (with Ge dissolved in it) as shown in figure 3. The lateral dimensions of the islands increase with increasing gold layer thickness while the island height depends on the deposition time at a given Ge deposition flux. In addition, the island growth rate is significantly higher (by approximately one order of magnitude) than the equivalent film growth rate observed under similar deposition conditions in the absence of gold. These characteristics are very similar to those found in the synthesis of semiconductor nanowires using the VLS approach. However, the growth of islands rather than wires should provide additional flexibility in the patteming of nanostructure arrays and their planar integration using conventional semiconductor processing. Experiments are underway to further reduce island dimensions and to develop patterning routes for island arrays. 


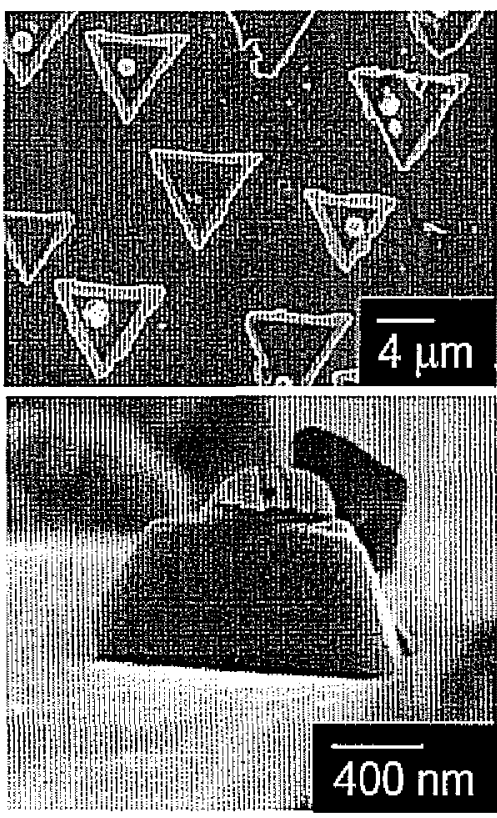

Figure 3: Ge islands. The top scanning electron micrograph is a top view intage of the islands grown using a starting Aulayer thickness of $3 \mathrm{~nm}$. The bottom nicrograph shows an island grown using a starting layer thickness less than $1 \mathrm{~nm}$. The round features on the island terraces are solidified Au-rich droplets.

\section{Publications}

D. M. Isaacson and O. D. Dubon, "Strain Symmetrization and Epitaxial Stabilization of Gey(SnxSi1-x)1-y Alloy Films on Ge (001)," LBNL report, in preparation.

\section{Holographic Imaging with X-rays: Fluorescence and Fourier Transform Holography}

Principal Investigators: Charles Fadley and Malcolm Howells

Project No.: 00030

\section{Project Description}

The goal of this project is to develop two promising new techniques for imaging matter in three dimensions using $\mathrm{x}$-rays. The first technique is hard $\mathrm{x}$-ray fluorescence holography (XFH), which has already demonstrated the ability to image atomic positions in three dimensions with accuracies of $\sim 0.2$ angstrom over a range of about 10 Angstroms around a given emitter. XFH will be extended to provide 0.1 -angstrom resolution over larger spatial regions and to yield enhanced elemental and magnetic sensitivity. The second technique is soft $\mathrm{x}$-ray holography and diffraction imaging (SXH/DD), which currently.is able to image wet life-science samples at 50-nanometer resolution in two dimensions. This will be further developed to yield ultimate resolutions of $\sim 10$ nanometers in three dimensions.

To accomplish this, an existing $x$-ray fluorescence holography experimental station will be upgraded in several ways for use at the Advanced Light Source (ALS) on bend-magnet beamlines (9.3.1 and 11.3.1), with the aim to place this system on a superbend or wiggler beamline in the future. The $\mathrm{x}$-ray energy discrimination and detection will be via high-speed solid-state detectors developed with special Berkeley Lab expertise. Experiments will be performed in both the direct (singleenergy) and inverse (multi-energy) XFH modes, as well as with resonant absorption, to enhance contrast, clemental sensitivity, and magnetic sensitivity. An existing soft $x$-ray holography experimental station will also be modified to permit the use of ALS beamline 9.0.1 for SXH/DI with a complex reference object (e.g., a random array of ultrasmall pinholes) and via speckle diffraction pattern analysis. New theoretical methods for inverting both hard $x$-ray and soft $x$-ray holograms/diffraction patterns to yield images will also be explored. There is a high degree of synergism between the two aspects of this work.

\section{Accomplishments}

\section{$X$-Ray Fluorescence Holography (XFH)}

In this past year, we have further developed the XFH experimental system and carried out preliminary experiments with it. Using this system, it has proven possible to reliably measure holograms with modulations of only a few tenths of a percent for several materials. These materials include simple structures such as $\mathrm{MnO}$ in (001) surface orientation and vanadium in (111) orientation, and more complex structures that will be further studied in the future: a colossal magnetoresistive (CMR) material $\mathrm{La}_{1-x} \mathrm{Sr}_{x} \mathrm{MnO}_{3}$ and a fivefold-symmetric quasicrystal (AIPdMn).

Some of the experimental data obtained was used for testing new XFH reconstruction algorithms. We have successfully demonstrated holographic imaging of diffraction structure factors and also shown that "superresolved" holographic reconstructions can be obtained by using iterative deconvolution methods. The latter study also compared several possible algorithms for obtaining enhanced resolution via $\mathrm{XFH}$, including some suggested 
by SXH/DI studies, and has paved the way for future developments in this field. Some results of this work are shown in Figs. (a)-( $f$ ), and two publications resulting from this work have appeared in Physical Review B. We have also shown theoretically that the use of resonantly-excited holograms should make it possible in future experiments to directly identify the neighbors to a given atom, thus yielding "atomic imaging in true color", with a publication appearing in Physical Review B on this as well.

Beyond this theoretical work, we have improved our experimental system in several ways. The main limitation of the first experimental setup was the energy range of beamline 9.3.1 at the ALS: only the region between 6 and $7 \mathrm{keV}$ was utilizable for $\mathrm{XFH}$, with energies up to about $12 \mathrm{keV}$ (corresponding to a wavelength of 1 angstrom) being optimal for XFH work. This limitation affects the number of elements that can be utilized and the ultimate resolution of the reconstructed holographic image. One of the first priorities from the experimental point of view was thus to adapt the set-up originally designed to work in an in-vacuum soft- $x$-ray chamber to an in-air hard-x-ray experimental hutch on beamline 11.3.1. This work has been accomplished, and some first data have been obtained on this beamline at higher incident $\mathrm{x}$-ray energies.

An additional instrumental improvement was to arrange for temperature-dependent studies at up to about $600 \mathrm{~K}$, while at the same time still being able to rotate the sample at $3600^{\circ}$ per second for noise-reducing signal averaging in the XFH measurements. This was accomplished by using a fixed shielded heater coil irradiating the back of the sample, and monitoring temperature with an infrared pyrometer looking at the sample surface (as crosscalibrated with the sample at rest using a thermocouple). We successfully tested the system on beamline 9.3.1, and adapted it to beamline 11.3.1. Some first temperaturedependent data obtained for a CMR sample (discussed further below) is currently being analyzed.

The only remaining limitation in the experimental system is that illumination of the solid state detector by a Bragg reflection from the sample can cause the detector to become saturated, making the hologram more difficult to interpret. A new detector system using a graphite analyzer is being planned to surmount this probtem, with support from the ALS for its construction.

A principal experimental project now underway with the improved system is to study the behavior of the $\mathrm{Mn}$ nearest neighbors in a newly-observed temperaturedependent electronic phase transition of a CMR material $\mathrm{La}_{1-\pi} \mathrm{Sr}_{x} \mathrm{MnO}_{3}(\mathrm{x}=0.3$ and 0.4$)$. This transition, which occurs $50-100 \mathrm{~K}$ above the Curie temperature, has been seen via soft $x$-ray spectroscopic measurements at the ALS (esp. core and valence photoemission), and it involves a dramatic change in the magnetic moment of $\mathrm{Mn}_{\text {r }}$ as well as the charge state of the other constituents ( $\mathrm{La}, \mathrm{Sr}$, and $\mathrm{O}$ ). The spectroscopic data also suggest a possible separation of electronic phases as temperature is raised. Temperature-dependent XFH will now be used to determine whether the electronic structure change in going from one phase to another as temperature is raised is also accompanied by significant atomic position changes. This aspect of the project addresses some key issues in the broader field of strongly correlated materials research, including also high-temperature superconductors.

\section{Soft $X$-Ray Holography (SXH) and Diffractive Imaging (DI)}

The aim of this project is to reconstruct images of nanostructures from their diffuse soft- $x$-ray transmission speckle (diffraction) patterns, and it has evolved from initial studies using a more holographic Fourier-transform method. The phase problem in imaging from speckle is solved using the Fienup oversampling method. During FY 2001 we developed a vacuum chamber with monochromator, pinholes, sample, and detector position adjustments. We then concentrated on sample preparation and the development of an off-axis zone plate monochromator for high-power undulator radiation.

We have now built a new monochromator that is able to sustain the high power undulator radiation, by coating the SiN substrate with $A l$ to aid in dispersing the heat, while not significantly increasing the absorption. With this improved setup, we were able to concentrate on a suitable test sample for image reconstructions at about $10 \mathrm{~nm}$ resolution.

Some experimental results are shown in Figures (g) and (h) below. Three experimental conditions must be met: the object to be imaged must be small (less then about $500 \mathrm{x}$ 500 resolution elements), isolated, and consist of strong scatterers with features with sizes of the order of the required resolution. We have used 50 nanometer gold balls dispersed from solution onto a small ( $2 \times 2$ micron $\left.^{2}\right)$ SiN window as a test sample. Better samples were then prepared at Livermore using an atomic force microscopy (AFM) to ensure isolation of the object. The resulting images are the first high resolution $(-10$ nanometers) single-particle (non-crystalline) $x$-ray images to be successfully reconstructed from such speckle data alone. The support (boundary) of the object needed to solve the phase problem, was obtained from the support of the autocorrelation function. This work with the AFM also showed the power of "prepared objects," such a single gold ball near a cell, for tomographic imaging. This work has been submitted to Physical Review Letters. 

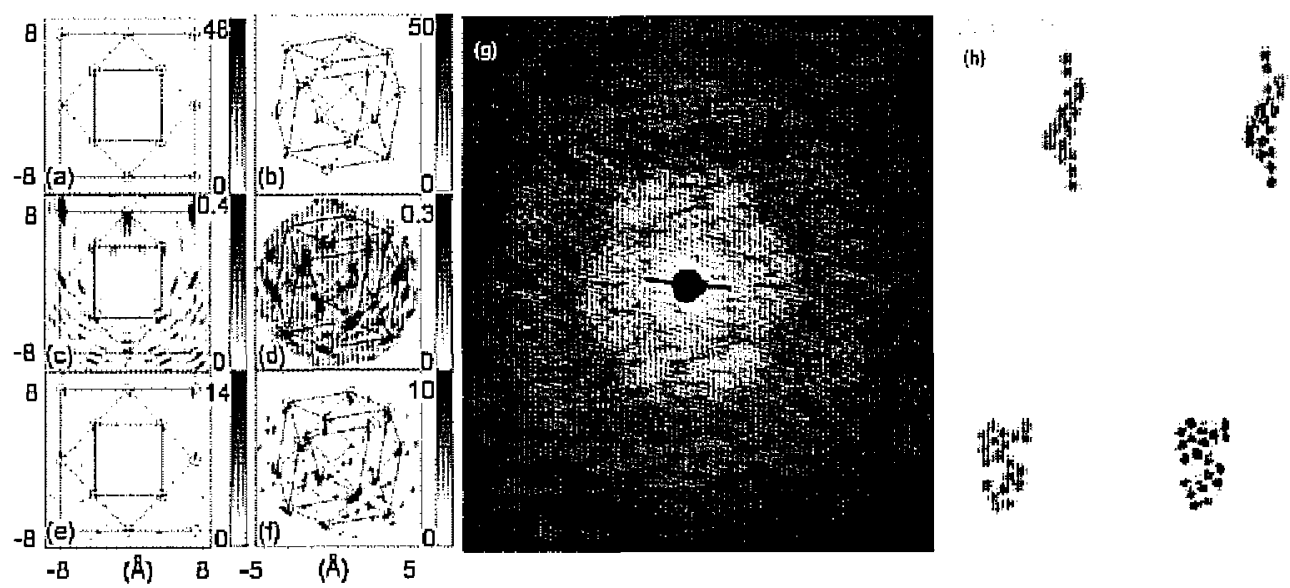

Figure 4: $(a)$-(f) Comparison between $x$-ray fluorescence holographic inages obtained by the standard holographic reconstruction algorithm and an improved iterative deconvolution method: (a),(b)--Original charge distributions (a) $X Z$ plane and $(b) 3 D$ view: $(c),(d)$--Intages corresponding to $(a),(b)$ from the standard holographic reconstruction. (e),(f)--Corresponding images from the iterative deconvolution method. The greyscale shows the maximum and minimum values. $(g)-(h)$ Restults from soff $x$-ray inaging via soft $x$-ray holography/diffraction imaging using speckle patterns: $(g)$-Soft $x$-ray transmission speckle pattern at $588 \mathrm{eV}$ from a cluster of gold balls with $50 \mathrm{~nm}$ diameter. The first minimum in the pattern occurs at $\sin \theta / \lambda=1.394 / d$. ( $h$ ) Comparison of reconstructed soft $X$-ray image (right) and SEM images of the same gold ball clusters (left). Each ball hats a diameter of $50 \mathrm{~nm}$. The $X$-ray image was reconstructed from $2.5 \mathrm{am}$ diffraction data alone.

\section{Publications}

S. Marchesini et al, "Holographic Analysis of Diffraction Structure Factors," Phys. Rev. B, 66, 094111 (4 pages) September 1, 2002.

hitp://ink.aps.org/abstract/PRB/v66/e094111

S. Omori et al, "Resonant $x$-ray Fluorescence Holography: Three-dimensional Atomic Imaging in True Color," Phys. Rev. B, 65, 014106 (5 pages) January 1, 2002. http://ink.aps.org/abstract/PRB/v65/e014106

M. Tegze et al., Comment on "X-ray Absorption Holography," Phys. Rev. Lett., 89, 279601, December 30, 2002. http://linkaps,org/abstract/PRL/v89/e279601

S. Marchesini, C. S. Fadley, "X-ray Fluorescence Holography: Going Beyond the Diffraction Limit," Phys. Rev. $B$, in press.

M. Howells et al., "Off-axis Zone Plate Monochromator for High-power Undulator Radiation," SPIE 4783, September 2002.

$\mathrm{H}$. He et al., "Inversion of X-ray Diffuse Scattering to Images Without Secondary Imaging," Phys. Rev. Lett., submitted for publication.

M. R. Howells, "X-ray Microscopy by Phase-retrieval Methods at The Advanced Light Source," XRM, July 29 August 2, 2002, Grenoble, France, Journ. de Phys. IV, submitted for publication.
H. He, et al., "Experimental Lensless Soft-X-ray Imaging Using Iterative Algorithms: Phasing Diffuse Scattering," Acta Crys. $A$, in press.

\section{Self-Assembling Arrays of Nanocrystals Templated by Cytoskeletal Proteins}

Principal Investigators: Matthew Francis

Project No:: 02034

\section{Project Description}

Actin is a remarkably versatile structural protein, serving both as a major component of the cytoskeleton and as the central scaffold of the muscle contractile apparatus. The utility of actin as a structural building block arises from its ability to form helical polymers that maintain their rigidity over lengths in excess of $10 \mu \mathrm{m}$ (in comparison, double stranded deoxyribonucleic acid (DNA) displays this property for only ca. 50 nanometers). Since these filaments are readily produced in vitro when the protein monomers are placed under the proper concentration, salt, and temperature conditions, modified actin subunits could 
provide a new method for arranging attached materials into periodic linear arrays through self-assembly (e.g. 2 in Figure 5a). It is anticipated that these arrays could serve as useful components of optical devices, and could be used to prepare nanoscale circuits after the subsequent deposition of additional metals.

To this end, this project focuses on (1) the design of a modular coupling strategy for the attachment of inorganic materials to protein surfaces, (2) the synthesis of watersoluble, uncharged nanocrystals with the appropriate functional sites for protein attachment, (3) the synthesis of actin conjugates modified in specific locations that do not interfere with self-assembly, (4) polymerization of the resulting conjugates into linear arrays, and (5) deposition of elemental silver along the template strands to yield metallic nanoscale conductors ( 3 in Figure 5a).

\section{Accomplishments}

The key to the success of this process is the design of a modular coupling method for the attachment of a variety of materials, including gold nanocrystals, to protein surfaces. For this purpose, we have chosen to use hydrazone formation reactions, as these linkages can be readily formed under physiological conditions that will not denature the protein components. Thus, initial experiments have focused on the preparation of actin molecules bearing pendant ketones, component 4 , and the synthesis of gold nanocrystals possessing hydrazide functional groups, component 5 as in Figure 5b.

Although there are many established preparations for watersoluble gold nanocrystals, these methods typically yield particles with charged surface functional groups (i.e. citrate or sulfonated phosphines). While these nanocrystals have been widely used in immunogold labeling procedures, the variable charge states of the particles interfere with many protein purification techniques, and can lead to agglomeration through nonspecific interactions with charged protein functional groups. In order to avoid these difficulties, we have developed a procedure for the preparation of goid nanocrystals possessing an uncharged passivation layer terminated with hydroxyl groups. Thus, the method of Hutchison and coworkers was used to prepare $A u_{10 t}$ clusters, and the surface ligands of these particles were exchanged with aliphatic mercapto-alcohols of various lengths. The size of the particles was determined to be $1.95 \pm 0.5$ nanometers by electron microscopy, and no changes in the size distribution were observed upon ligand exchange. In order to introduce the hydrazide functional groups, the ligand exchange method of Fitzmaurice was. followed using a mixture of mercapto-alcohols and mercapto-hydrazides. By altering the hydrazide/alcohol ratio, the number of hydrazides on the nanocrystal surfaces can be controlled.

Monomeric actin has been obtained from rabbit muscle tissue following literature procedures, and the protein has been purified using gel filtration. The protein consists of four subdomains of uneven size arranged in a square pattern; fiber assembly aligns these monomers in a back-toback orientation with a periodicity of 5.5 nanometers. As one of the subdomains protrudes substantially from the helical core in these fibers, a cysteine residue on this portion of the protein has been identified in previous studies as an exposed position for modifications that do not interfere with polymerization. This tolerance to substitution provides an ideal location for the introduction of ketones, as the subsequently attached nanocrystals should exert a minimal impact on the assembled fiber structure.

Thus, ketone attachment has been accomplished by alkylating cys 374 with iodoacetamides. We have prepared substrates for this purpose using an efficient synthetic route, yielding water-soluble iodoacetoamides in excellent yield. The ethylene glycol portion introduces a water-soluble spacing group that can be adjusted in length as necessary. After polymerization of the actin monomers, the desired cysteine was alkylated with the iodoacetamide to afford bioconjugate component 4 . The presence of the new functional group was established using a ketone-selective Western blot assay. Current efforts are directed toward the coupling of the nanocrystals to this new functional group through hydrazone formation. 


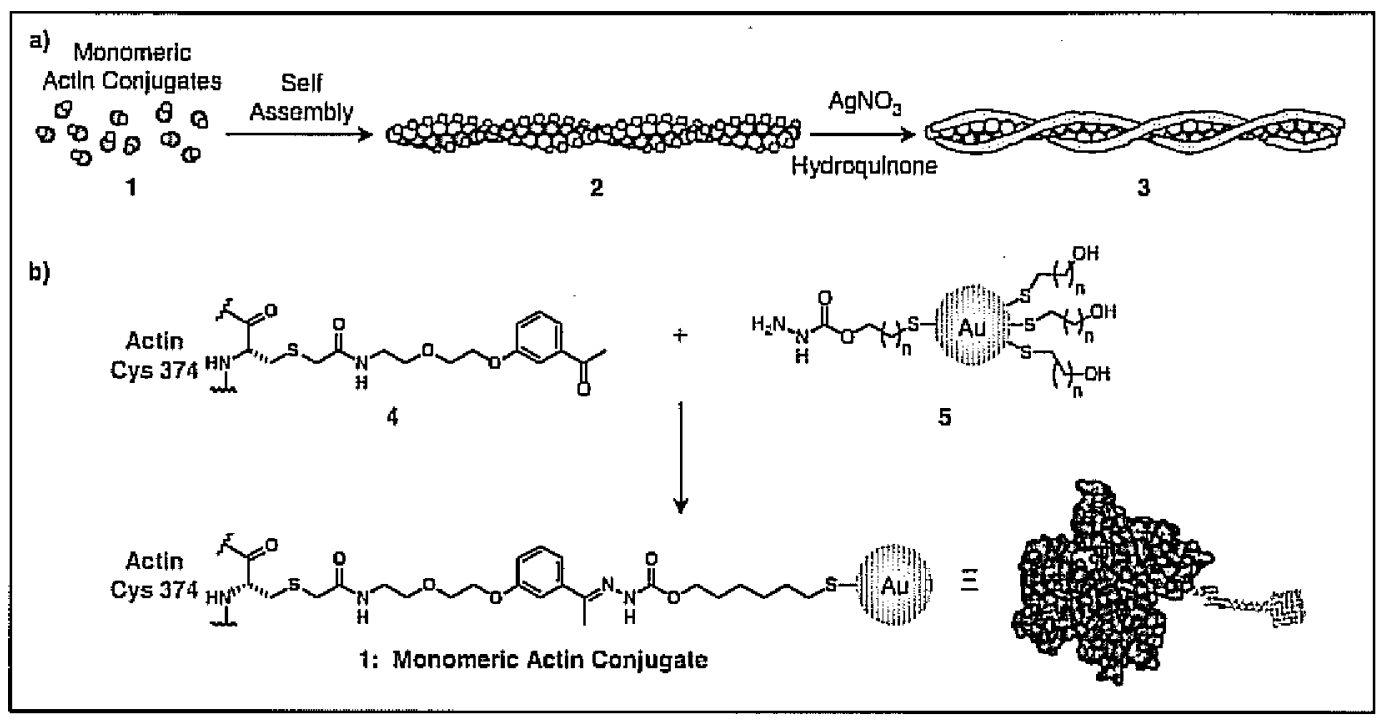

Figure 5: Synthesis of linear nanocrystalline arrays through actin polymerization. (a) After the self-assembly process, arrays such as 2 could be converted into nanoscale conductors by using the gold nanocrystals to seed the deposition of silver metal (3). (b) The actin-gold conjugates will be prepared using a modular coupling strategy. Components 4 and 5 have been synthesized, and are currently being joined though hydrazone formation.

\section{Investigation of High-pressure Surface Reactions with a Molecular Beam of Reaction Products}

Principal Investigators: D. Frank Ogletree and Miquel Salmeron

Project No:: 02035

\section{Project Description}

Fundamental studies of surface processes and reactions at high pressure have been outside the realm of surface science-the soncalled "pressure gap". One step in closing the pressure-gap was taken at Berkeley Lab over the last three years with our development of a high-pressure photoelectron spectroscopy (HPPES) instrument operating at the Advanced Light Source (ALS) beamline 9.3.2.

Surface reactions can be investigated with unprecedented detail at pressures in the Torr range using FPPES to analyze the composition and electronic structure of the catalyst surface and its adsorbed molecules, while simultaneously analyzing the gas-phase reactants and products within a molecular mean-free-path of the surface. Photoemission under high pressure conditions can detect gas-phase molecules at partial pressures of $\sim 50 \mathrm{~m}$ Torr.

\section{Accomplishments}

The HPPES instrument has been used to investigate several catalytic systems under reaction conditions during the last year.

Work on the partial oxidation of methanol to formaldehyde over copper oxide has been done. While the industrial reaction takes place at $1 \mathrm{Bar}$, most of this pressure is an inert carrier gas. The partial pressures of reactant and product molecules and the catalyst temperature range in the industrial reaction are the same as in our HPPES study at the ALS. The oxidation state of the catalyst surface can be monitored as a function of temperature. As temperature increases, the electronic structure of the copper surface changes from an oxide with a band gap to metallic, correlated with a decrease in $\mathrm{CO}_{2}$ production due to total oxidation and an increase in $\mathrm{CH}_{2} \mathrm{OH}$ production, the desired partial-oxidation product (see figure 6). The effects of feed-gas stoichiometry and the transient response of the catalytic system were also investigated.

Another study investigated the partial oxidation of n-butane to maleic anhydride (a polyester resin precursor) over an industrial vanadium phosphorous oxide catalyst. This and the previous methanol oxidation study were carried out in collaboration with the inorganic chemistry department of the Fritz Haber Institute (Prof. R. Schogl, Berlin, Germany). 
Work on the coadsorption of $\mathrm{CO}$ and NO on $\mathrm{Rh}(111)$ was also done. This is a model reaction for automotive exhaustgas catalytic conversion. At high pressures $\mathrm{CO}$ adsorbs in top and three-fold hollow sites on $\mathrm{Rh}(111)$. These adsorption sites can be distinguished by high-resolution photoemission spectroscopy with a synchrotron photon source. Preliminary results show that when even small amounts of $\mathrm{NO}$ are added to the $\mathrm{CO}$ gas, NO molecules rapidly displace $\mathrm{CO}$ from the 3 -fold adsorption sites on $\operatorname{Rh}(111)$, while the top sites continue to be occupied by $\mathrm{CO}$ molecules.
A new investigation of the interaction of the $\mathrm{NaCl}(001)$ surface with water vapor has been initiated. By reducing the substrate temperature slightly below $0^{\circ} \mathrm{C}$, it is possible to increase the water vapor partial pressure continuously up to the deliquescence point, where the salt crystal is converted to a saturated brine solution. This process, which has important implications for environmental chemistry, produces irreversible changes in the salt surface composition. The salt investigations have been carried out in collaboration with Prof. John Hemminger (Chemistry, University of California, Irvine).

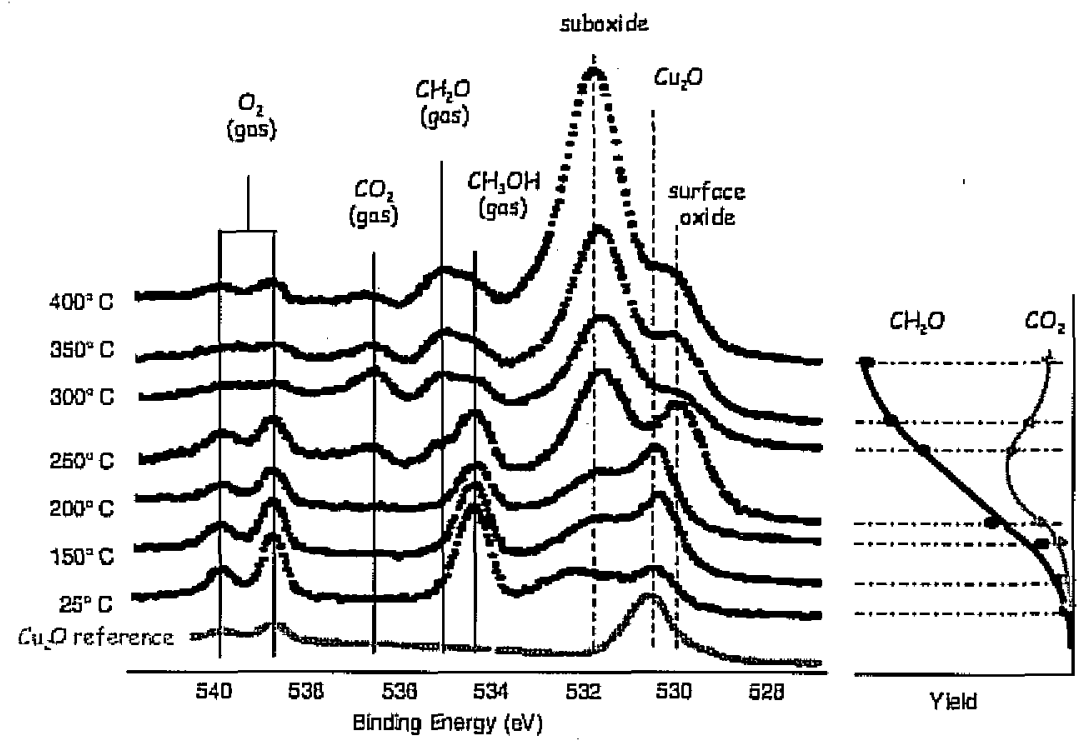

Figure 6: Temperature-dependent HPPES XPS spectrum of copper oxygen during partial oxidation of methanol showing gas phase reactants $\left(\mathrm{O}_{2}, \mathrm{CH}_{3} \mathrm{OH}\right)$, products $\left(\mathrm{CH}_{2} \mathrm{OH}, \mathrm{CO}_{2}\right)$ and copper-oxygen peaks (indicated by vertical dashed lines). The inset graph at right shows product yield measured by mass spectroscopy, which correlates well with the direct XPS measurements above the sample surface. Reaction pressure 0.4 Torr, $\mathrm{CH}_{3} \mathrm{OH}: \mathrm{O}_{2}$ ratio 3:1.

\section{Publications}

M. Havecker, R.W. Mayer, A. Knop-Gericke, H. Bluhm, E. Kleimenov, A. Liskowsi, D. Su, R. Follath, F. Requejo, D. F. Ogletree M. Salmeron, J.A. Lopez-Sanchez, J. K. Bartley, G. J. Hutchings, and R. Schlögl, "lin situ Investigation of the Nature of the Active Surface of a Vanadyl Pyrophosphate Catalyst Curing n-butane Oxidation to Maleic Anhydride," November 2002, submitted for publication.

H. Bluhn, M. Hävecker, A. Knop-Gericke, V.I. Bukhtiyarov, D.F. Ogletree, M. Salmeron, R. Schlögl, " $I n$ situ XPS Investigation of the Methanol Oxidation Over Copper," AVS National Symposium, Denver, Colorado USA, November 2002.

http://www.avs.org/symposium2002/paper.asp?abstractID= 772
M. Salmeron, "Ice-vapor Interfaces by Photoelectron Spectroscopy," APS March Meeting 2002 (invited), Indianapolis, IN, USA, March 2002. http:/www.aps.org/meet/MAR02/baps/abs/S7860001.html

D. F. Ogletree, "Closing the Pressure Gap: Photoemission Above $10 \mathrm{mBar}$," XXIII CBRAVIC Brazilian Congress of Vacuum Science and Industrial Applications, (invited) Floranopolis, Brazil, July 2002. 


\section{Photoemission Study of Magnetic Quantum Well Interaction}

\author{
Principal Investigators: Zi Qiang Qiu
}

Project No.: 02036

\section{Project Description}

Quantum Well (QW) states are now well recognized as a general property that plays the key role in generating spin dependent phenomena in magnetic nanostructures. In the last a few years, it has been shown that the formation of QW states is responsible for the oscillatory magnetic coupling and the giant magnetoresistance. At current stage, most effort has been focused on the understanding of a single QW system and its relation to the magnetic interlayer coupling. In this project, we go beyond the single QW to investigate the coupling of QW states from different layers, and then the new magnetic properties in these nanostructures. Electronic states of the $\mathrm{QW}$ structures will be measured using angle resolved photoemission spectroscopy (ARPES) at bearnline 7.0.1.2 and magnetic properties will be measured using Photo-Electron Emission Microscope (PEEM) at beamline 7.3.1.1. Then the direct connection between the magnetic behaviors and the electronic confinement will be established in magnetic nanostructures.

\section{Accomplishments}

Double quantum well (QW) system of $\mathrm{Cu} / \mathrm{Co}(1 \mathrm{ML}) / \mathrm{Cu}$ grown on $\mathrm{Co} / \mathrm{Cu}(100)$ were investigated by photoemission spectroscopy. Energy spectra of the valence band from one of the $\mathrm{Cu} \mathrm{QWs}$ have been measured as a function of the other $\mathrm{Cu}$ QW thickness. The results show strong resonance between the two Cu QW states across the I monolayer $\mathrm{Co}$. In particular, we observe that quantum well coupling removes degeneracy of the two $\mathrm{Cu} \mathrm{QW}$ states, resulting in a state crossing effect. Phase accumulation model is developed to explain these observations.

$\mathrm{Ni} / \mathrm{Fe} / \mathrm{Co} / \mathrm{Cu}(100)$ system was investigated. Unlike bulk bcc ferromagnetic $\mathrm{Fe}$, the epitnxial grown fcc $\mathrm{Fe}$ in this structure is antiferromagnetic in the 4-12 monolayer thickness range. By analyzing element specific domain images, we found that there exists oscillatory interlayer coupling between the $\mathrm{Co}$ and $\mathrm{Ni}$ layers across the fcc $\mathrm{Fe}$ layer. With the thickness and temperature dependent studies, we found strong evidence that the fcc Fe layers at both the $\mathrm{Fe} / \mathrm{Co}$ and $\mathrm{Fe} / \mathrm{Ni}$ interfaces are ferromagnetic live in the 4-12monolayer thickness range, and that the live layer at the $\mathrm{Fe} / \mathrm{Ni}$ interface disappears as the temperature exceeds the Curie temperature of the Ni layer. Moreover, the oscillatory interlayer coupling between the $\mathrm{Co}$ and $\mathrm{Ni}$ layers results in an oscillation of the Ni layer Curie temperature

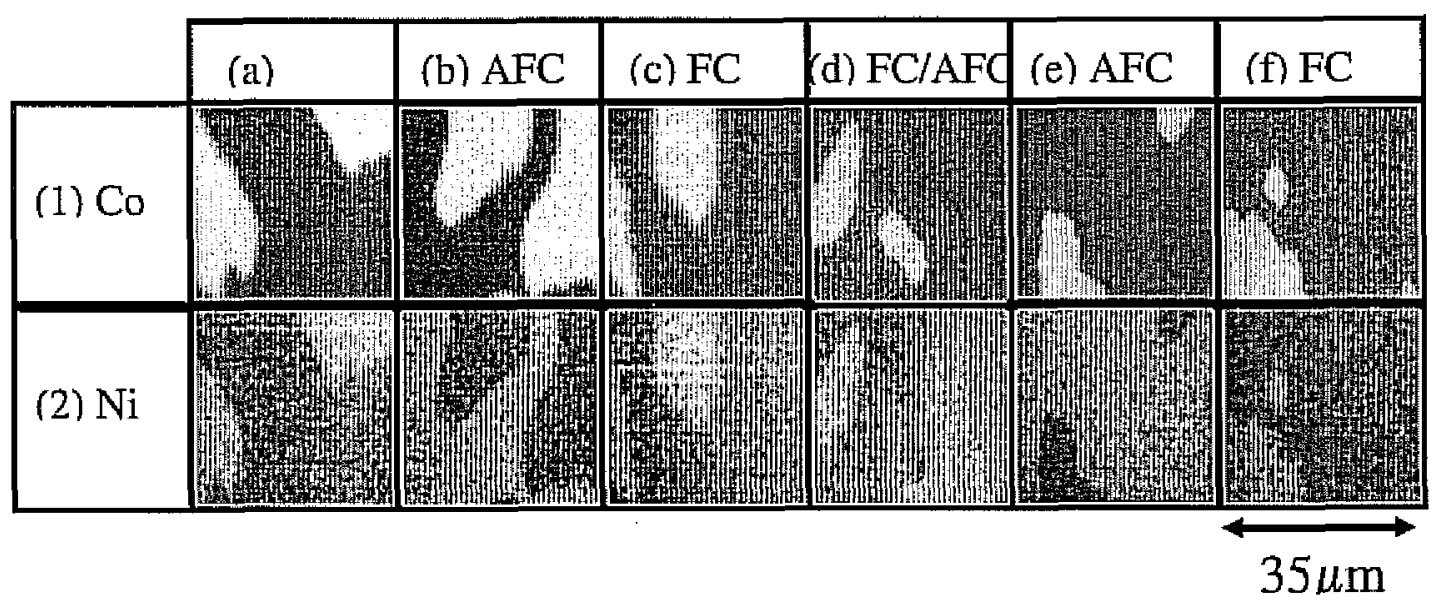

Figure 7: Magnetic domain images of $\mathrm{Cu}(1 /$ monolayer $[\mathrm{MLJ}) / \mathrm{Ni}(4.7 \mathrm{ML}) / \mathrm{Fe} / \mathrm{Co}(1 \mathrm{OML}) / \mathrm{Cu}(100)$. The rows represent (I) Co and (2) $\mathrm{Ni}$, and the columns are for (a) $\mathrm{dFe}=2.0 \mathrm{ML},(\mathrm{b}) \mathrm{dFe}=4.3 \mathrm{ML}$, (c) $\mathrm{dFe}=7.3 \mathrm{ML}$, (d) $d F e=8.9 \mathrm{ML}$, (e) $d F e=10.3 \mathrm{ML}$, and $(f) \mathrm{dFe}=12.6 \mathrm{ML}$. Oscillatory interlayer coupling can be clearly seen by looking at the relative domain color of the $\mathrm{Ni}$ and Co films. 


\section{Publications}

Z.Q. Qiu and N.V. "Smith, Quantum Well States and Oscillatory Magnetic Interlayer Coupling," J. of Physics: Condensed Matter, 14 (2002) R169.

W.L. Ling, Eli Rotenberg, H.J. Choi, J.H. Wolfe, F. Toyama, S. Paik, N.V. Smith, and Z.Q. Qiu, "Double Quantum Well States in $\mathrm{Cu} / \mathrm{Co} / \mathrm{Cu}$ Grown on $\mathrm{Co}(001)$," Phys. Rev. B, 65, 113406 (2002)

Y.Z. Wu, C. Won, A. Scholl, A. Doran, F. Toyoma, X.F. Jin, N.V. Smith, and Z.Q. Qiu, "Interfacial Magnetism of fcc Fe and the Effect of the Oscillatory Interlayer Coupling on the Ni Magnetic Properties in Ni/Fe/Co/Cu(100), "P/lys. Rev. B, 65, 214417 (2002).

Hyuk J. Choi, W.I. Iing, A. Scholl, J.H. Wolfe, S. Anders, U. Bovensiepen, F. Toyama, S. Paik, F. Nolting, and Z.Q. Qiu, "Spin Reorientation Transition in Magnetically Coupled Fe/Cu/Ni/Cu(001)," Phys. Rev. B, 66, 014409 (2002)

Y.Z. Wu, C.Y. Won, E. Rotenberg, H.W. Zhao, F. Toyoma, N.V. Smith, and Z.Q. Qiu, "Dispersion of Quantum Well States in $\mathrm{Cu} / \mathrm{Co} / \mathrm{Cu}(001), "$ Pliys. Rev. B, Accepted for publication.

C. Won, Y,Z. Wu, N. Kurahashi, K.T. Law, H.W. Zhao, A. Scholl, A. Doran, and Z.Q. Qiu, "Evidence of the Oscillatory Magnetic Anisotropy in $\mathrm{Ni} / \mathrm{Co} / \mathrm{Ni} / \mathrm{Cu}(100)$," Phys. Rev, B, submitted for publication.

\section{Superconducting $\mathrm{MgB}_{2}$ Nanofibers and Nanotubes}

Principal Investigators: Peidong Yang

Project No.: 02037

\section{Project Description}

Whether there is a limit to how thin a superconducting wire can become before losing its superconducting character is both an important fundamental question and a practical issue in the miniaturization of superconducting electronic circuits. Past studies of superconducting nanowires have focused mainly on low transition temperature $\left(T_{c}\right)$ systems (below 10K) such as $\mathrm{In}, \mathrm{Pb}$, and MoGe due to the difficulty in synthesizing/fabricating superconducting nanowires with higher $\mathrm{T}_{\mathrm{c}}$. The newly discovered $\mathrm{MgB}_{2}$ superconductor, with its remarkably high transition temperature $\mathrm{T}_{\mathrm{c}}-39^{\circ} \mathrm{K}$ and simple crystal structure, represents a promising system to explore the effects of dimensionality and size on the superconductivity. $\mathrm{MgB}_{2}$ is a hexngonal $\mathrm{AlB}_{2}$-type compound, consisting of alternating hexagonal $\mathrm{Mg}$ layers and graphite-like B layers. In fact, graphite is iso-electronic with $\mathrm{MgB}_{2}$. In this proposal, we propose to research the possibility of $\mathrm{MgB}_{2}$ nanotube and nanofiber formation and characterize their size- and dimensionality-dependent superconducting properties. This research offers important superconducting nanoscale building blocks for the future nanoelectronic applications.

A home-buitt high-temperature laser-deposition system will be used in this study. Two possible routes will be adopted during the synthetic process. One is the vapor-liquid-solid nanowire growth process that has been developed in this lab, the other one is the catalytic nanotube growth process, which was generally used to produce carbon and boron nitride nanotubes. The first approach will yield solid-core nanofibers while the second approach could produce hollow nanotubes. Both processes will use catalysts with specific compositions. It should be feasible to synthesize these two different nanostructures under different conditions considering the unique crystal structure of the $\mathrm{MgB}_{2}$ (being layered and isotropic at the same time). Theoretical calculation on the stability of $\mathrm{MgB}_{2}$ nanotubes and their electronic structures will provide further guidance for this work. Important synthetic parameters that will be explored include different catalysts (e. g. Fe, Ni, Co, Mo etc.), reaction temperature and pressure. Scanning electron microscopy, transmission electron microscopy, composition analysis, magnetization measurement and electron transport measurement will be used to thoroughly characterize these nanostructures so that the correlation between the size, dimensionality, curvature of the nanostructures and their superconducting properties can be extracted correctly.

\section{Accomplishments}

Superconducting $\mathrm{MgB}_{2}$ nanowires were synthesized using a two-step vapor transport reaction process. Amorphous boron nanowires were first synthesized using a chemical vapor transport process. The boron nanowires were then transformed into $\mathrm{MgB}_{2}$ nanowires through a solid-vapor reaction process. The $\mathrm{MgB}_{2}$ nanowires generally have diameters of $50-400 \mathrm{~nm}$ and lengths up to ten micrometers. Magnetization measurement indicates these nanowires are superconducting with transition temperature of $-33^{\circ} \mathrm{K}$. The $\mathrm{MgB}_{2}$ nanowire represents a promising system to explore the effects of dimensionality and size on the superconductivity and might be used as low-dissipation interconnects in nanoscale electronics.

Besides the amorphous $\mathrm{B}$ to $\mathrm{MgB}_{2}$ transformation studies, we have also explored numbers of different metals and found that $\mathrm{Pt}$ is the best catalyst for the VLS growth of pure single cтystalline boron nanowires. This initial study on 
boron nanostructure formation serves as the foundation for our continuing efforts in exploration of novel growth phenomenon including boron and metal boride nano-tubular growth. Difficulties inherent in the pure boron system are the formation of dense boron and metal boride pellets to prevent splashing of the material during ablation, the extremely high temperatures needed to form eutectics with metals and the ease at which boron incorporates impurity elements into solid-state crystal structures. These problems, particularly, the oxidation of the resulted nanostructures, were also encountered during the direct ablation of $\mathrm{MgB}_{2}$ and $\mathrm{AlB}_{2}$ targets. To avoid the potential oxidation and contamination problems, we have constrtcted a ultra-high vacuum (UHV)-pulsed laser ablation system. We are currently systematically investigating the nanostructures produced under different conditions (metals, temperatures and pressures) in this UHV system to examine the possibility of $\mathrm{MB}_{2}(\mathrm{M}=\mathrm{Mg}, \mathrm{Al})$ single crystalline nanowire and nanotube formation.

In addition to the above attempts on $\mathrm{MgB}_{2}$ nanotube formation through direct laser ablation, a different approach has been taken recently. This new approach uses carbon nanotubes or other semiconductor nanowires as physical templates for making single crystalline nanotubes, i.e., epitaxial casting. For example, uniform $\mathrm{GaN}$ nanotubes were synthesized using a chemical vapor deposition process. Well-facetted single crystalline $\mathrm{ZnO}$ nanowires were used as templates for epitaxial growth of thin $\mathrm{GaN}$ layers and were subsequently removed via thermal reduction and evaporation. These GaN nanotubes have inner diameters of $30 \mathrm{~nm}$ to $200 \mathrm{~nm}$, and the wall thicknesses of 5-50 nm. Transmission electron microscopy studies indicate that the nanotubes are single crystalline with a wurtzite structure and are oriented along the <0001> direction. These single crystalline wurtzite GaN nanotubes differ fundamentally from theoretically predicted $\mathrm{GaN}$ nanotubes, where a metastable graphitic GaN structure was proposed. This is, to the best of our knowledge, the first example of single crystalline nanotubes of materials without layered structure. Similar "epitaxial casting" approaches could be used to produce nanotubes of other materials including the $\mathrm{MB}_{2}(\mathrm{M}=\mathrm{Mg}, \mathrm{Al})$ systems.
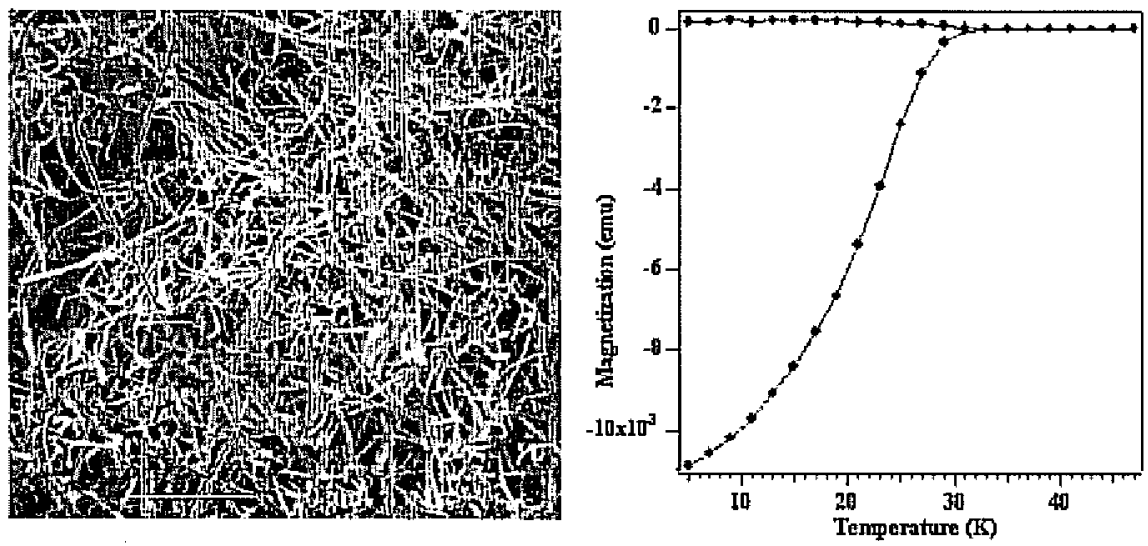

Figure 8: Scanning electron microscopy image of $\mathrm{Mg}_{2}$ nanofibers and magnetization of these $\mathrm{Mg}_{2} B_{2}$ nanowires as a function of temperature. Data are shown for measurements ander conditions of zero field cooling (ZFC) and field cooling (FC) at 100 Gauss.

\section{Publications}

Y. Wu, B. Messer, P. Yang, "Superconducting $\mathrm{MgB}_{2}$ Nanowires," Adv. Mater. 13, 1487, 2001

Y. Li, J. Wang, Z. Deng, Y. Wu, X. Sun, S. Fan, D. Yu, P. Yang, "Bismuth Nanotubes," J. Am. Chem. Soc. 123, 9904,2001

J. Goldberger, R. He, H. Choi, P. Yang, "Single Crystalline Gallium Nitride Nanotubes," Nature, submitted for publication. 


\section{Synthesis and Control of Molecular Machines}

Principal Investigators: Alex Zettl and Michael

Crommie

Project No.: 02038

\section{Project Description}

The main goal of this project is to develop new synthetic nanomachines and nanomotors from individual molecular components. Nanomachines will be designed and fabricated in a molecule-by-molecule fashion to perform specific mechanical tasks under a variety of operating conditions, greatly exceeding the operating parameter range of existing biological motors. Functional molecules will be assembled at surfaces using highly parallel selfassembly techniques as well as single-molecule manipulation. Scanned probe techniques will be used to characterize molecular machine operation at the angstrom level. Nanolithographic techniques will be combined with molecular deposition to fabricate and actuate electromechanical devices at the nanometer scale.

\section{Accomplishments}

We have made significant progress along a number of fronts involving NEMS (nanoelectromechanical systems) fabrication of molecular motors and local probe measurement of nanostructures. This work has tecently attracted longer term funding through a joint grant from the DOE.

Much of our effort over the last year was spent determining a viable methodology for utilizing multi-wall carbon nanotubes (CNT's) as key structural/electronic components in nanoscale machines. The idea here is to use the concentric shells of multiwall nanotubes as low friction bearing surfaces in electric motors. We have successfully demonstrated the deposition of CNT's on silicon oxide substrates, and have contacted these tubes with rigid bearing "mounts". We have also deposited three independent stators around the shaft and attached a rotor to the nanotube. The rotor has been successfully undercut in our fabrication process. These developments set the stage for the construction of a fully operational electric nanomotor.

In addition to the work on CNT's, we have optimized a home-made variable temperature scanning tunneling microscope or microscopy (STM) system and are now able to perform high-resolution spectroscopic studies of molecular systems on semiconducting and metallic surfaces. We have probed indium nanowires on clean $\mathrm{Si}(100)$ surfaces as a possible molecular machine scaffold and and also as a potential means for forming electrical contact to molecular assemblies. Indium nanowires only 1 atom wide were self-assembled at the $S i(100)$ surface and probed using STM spectroscopy. In order to understand the indium nanowire response, we first characterized the underlying $\mathrm{Si}(100)$ electronic structure. This resulted in the discovery of a new surface state feature in the $S i(100)$ density of states that we identify as a $\pi$-state band. We also found that Si surface-state electronic structure is very sensitive to local electric fields induced by the STM tip. This atomic-scale band-bending effect was characterized for different STM tip-sample distances (see Fig. 9)

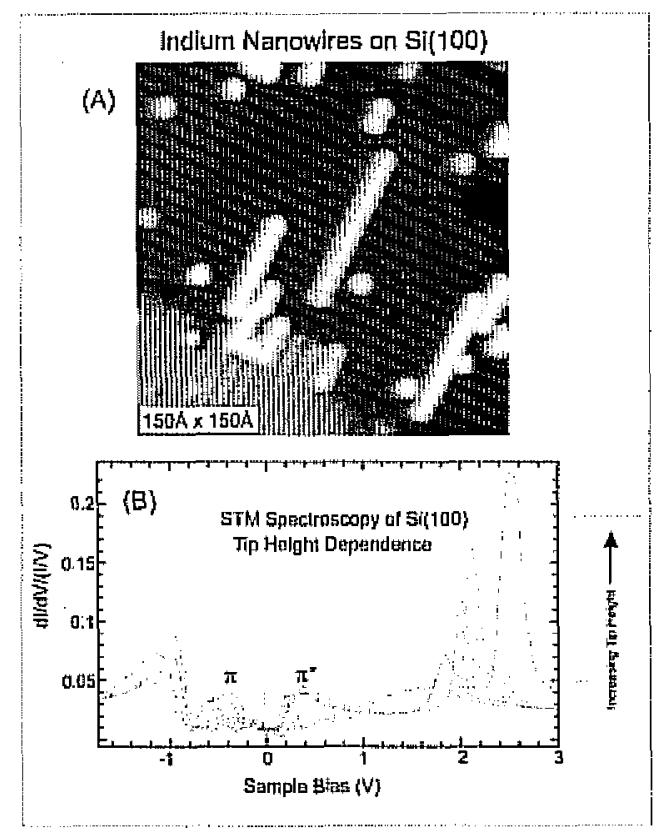

Figure 9: (A) $150 \AA$ x 150A STM inage of single-atomwide inditum nanowires on Si(100) surface.

(B) Result of STM spectroscopic measurentents on Si(100) surface. Si(100) $\pi$ and $\pi^{*}$ states are clearly visible, as well as band-bending effects (i.e., spectroscopic shifts) as STM tip position is changed.

\section{Publications}

K. Nagaoka, M. Comstock, A. Hammack, M. F. Crommie, "Atomic-scale Bandbending at the Si(100) Surface," December 2002, in preparation for submission to Physical Review B., Rapid Communications. 


\section{Nuclear Science Division}

\section{Effective Field Theory and Few- Nucleon Systems}

\author{
Principal Investigators: Paulo Bedaque \\ Project No.: 02039
}

\section{Project Description}

The goal of this project is to extend the effective field theory methods recently developed for the two-nucleon sector to systems involving three or more particles (including bulk matter). In particular, we aim at developing model independent, rigorous and precise calculations of processes involving electroweak currents on triton- $3 \mathrm{He}$.

More specifically, we plan to perform a third-order calculation of the triton decay that can then be applied to determine the short distance contribution needed for a $1 \%$ level calculation of neutrino-deuteron break up (relevant for Sudbury Neutrino Observatory (SNO\}) and proton-proton fusion (fundamental for solar models).

Due to the similarities between the physics of the lowenergy nucleon-nucleon interaction and that of some atomic species we will examine applications to the physics of atomic traps (especially close to a Feshbach resonance).

\section{Accomplishments}

Previously, the renormalization and power counting in the three-body system were understood only to leading order of the low energy expansion. In order to extract interesting physics, calculations should be done, at least, to third order, what corresponds to a precision level of a few percent (approximately, depending on the observable). We provided a very general argument estimating the influence of short distance nucleon-nucleon interaction on the low energy observables that can be encapsulated in a very simple rule. The three-body interactions are essentially promoted two orders as compared to the naïve dimensional analysis estimate. The leading three-body interaction, for instance, become a leading order effect, as previously known. The sub-leading three-body interaction appears at the third-order, not on fifth order, as dimensional analysis suggests, and so on. This enhancement applies only to interactions satisfying the Wigner SU(4) symmetry.
As a testing ground of this scheme we performed a thitd order calculation of elastic neutron-deuteron scattering. A few technical improvements had to be made for the numerical solution of the Faddeev equation. We confirmed the independence in relation to the arbitrary value of the cutoff used, the convergence of the low energy expansion and obtained results in agreement with the experiments available. More than that, we showed that our uncertainties are dominated now by the input parameters coming from the two-nucleon sector and that, consequently, these. calculations (or any other model calculation) cannot be improved until better measurements of these input parameters are made. However, the uncertainty implied by the low energy expansion seems to be enough to have an impact in processes of astrophysical and/or theoretical importance, in particular the triton beta-decay that can be used to calibrate neutrino-deuteron interactions.

Motivated by our interest in nuclear and neutron matter and by the explosive progress in the physics of atomic traps near Feshbach resonance we used some of the methods and ideas generated in the few-nucleon physics to the study of dilute gases. First we explored the influence of the threebody correlations on the thermodynamic properties of bosonic gases. In the dilute (virial) expansion, the first correction is determined entirely by the two-body scattering length, according to a classical result by Beth and Uhlenbeck. We computed the next correction, which describes the three-particle correlations. We found it to be a universal function of just one parameter. Contrary to our initial expectation no dramatic effects arise when a threebody bound state forms at threshold.

A more dramatic effect however, can be seen at very low temperatures, where the virial expansion is irrelevant. We found that that the effective three-body interaction terms, that is typically a small term, can be made arbitrarily large by fine-tuning the two-body scattering length, and become as important as the two-body interaction terms. Further, being repulsive, it can stabilize a system that would be otherwise unstable due to the attractive two-body interactions. Since two-body scattering lengths can be manipulated essentially at will close to a Feshbach resonance, this allows for the creation of a whole new class of self-bound quantum liquids, the only ones besides the two classical examples (helium liquid and nuclear matter). Moreover, the properties of this state can be computed, using effective theory techniques, from first principles. 


\section{Publications}

A. Bulgac, P.F. Bedaque, "Atomic-Molecular Condensates with Large Positive Scattering Length," Condensed Matter, cond-mat/0210217, October 2002, submitted for publication.

P.F. Bedaque, A. Bulgac, and G. Rupak, "Quantum Corrections to Dilute Bose Liquids," Condensed Matter, cond-mat/0209274, September 2002, submitted for publication.

P.F. Bedaque, G. Rupak, H.W. Griesshammer, and H.W. Hammer, "Low-Energy Expansion in the Three Body System to All Orders and the Triton Channel," Nuclear Theory, nucl-th/0207034, July 2002, Nuclear Physics A, accepted for publication.

P.F. Bedaque and G. Rupak, "Dilute Resonating Gases and the Third Virial Coefficient," Condensed Matter, condmat/0206527, June 2002, Physical Review B, accepted for publication.

P.F. Bedaque (LBNL, Berkeley), U. van Kolck, "Effective Field Theory for Few Nucleon Systems," Nuclear Theory, nucl-th/0203055, January 2002, Antntal Reviews on Nuclear and Particle Plysics, accepted for publication.

\section{Design of Digital Signal Processing Electronics for High-Resolution Radiation Detectors}

Principal Investigators: I-Yang Lee

Project No.: 01032

\section{Project Description}

This project was carried out to initiate the development of digital electronics and associated data processing software for future high-resolution radiation detectors, such as the GRETA (Gamma-Ray Tracking Array) project lead by the Nuclear Science division of Berkeley Lab. Specifically, the goal of this LDRD project is to design, manufacture and test an 8-channel, $100 \mathrm{MHz}$ analog to digital converter (ADC) board to (1) instrument the GRETA module prototype array and (2) to serve as a basis for a more complex 40-channel ADC board required for the full GRETA array. The board also meets the specifications outlined at the digital electronics workshop held at Argonne National Laboratory (2001) for a general-purpose digital signal processing board for the low-energy nuclear physics community.

Unlike commercially available ADC boards, specialized real-time signal processing is required on the board itself to perform real time data reduction, implement the complex trigger logic the system requires, and emulate the required elements of traditional analog systems. Furthermore, this board requires very high readout rates due to both the high detector counting rates $(20 \mathrm{kHz})$ and the large amount of data produced per gamma-ray interaction (100 bytes).

The board itself is designed around 8 commercial $100 \mathrm{MHz}$ pipeline ADC chips whose data streams are fed into a single Virtex 2 Field Programmable Gate Array (FPGA) which is responsible for all on board processing. This state of the art FPGA also contains internal memory buffers that are employed to allow for up to $10 \mu \mathrm{s}$ of latency for more flexible trigger configurations, The FPGA provides 8 leading edge discriminators for triggering and 8 constant fraction discriminators for amplitude independent signal timing. The energy of each signal is determined by application of a trapezoidal filter to the incoming signal. An adjustable window is also generated allowing one to extract only the part of the signal required for subsequent signal decomposition thus eliminating extraneous data early in the data acquisition process. Data is sent to the acquisition computer through a standard VERSA Module Eurocard (VME) bus allowing the card to be used not just with the GRETA project but also in the wider low-energy nuclear physics community.

\section{Accomplishments}

The design of the 8-channel board began in January 2002. Futl simulations of the complex VHSIC Hardware Description Language (VHDL) code that implement the above functions in the FPGA have been carried out successfully. Layout of the board was completed in September 2002 and three cards were manufactured and populated. Tests of the analog and digital sections of the card are currently being carried out by the board design engineers. These tests are proceeding on schedule and injected signals have been successfully processed by the board and read from the VME bus. We expect to carry out preliminary tests of the board with Ge detectors beginning in December 2002. Following successful tests, 15 of these boards will be integrated into a 120-channel data acquisition system for the GRETA module prototype. 


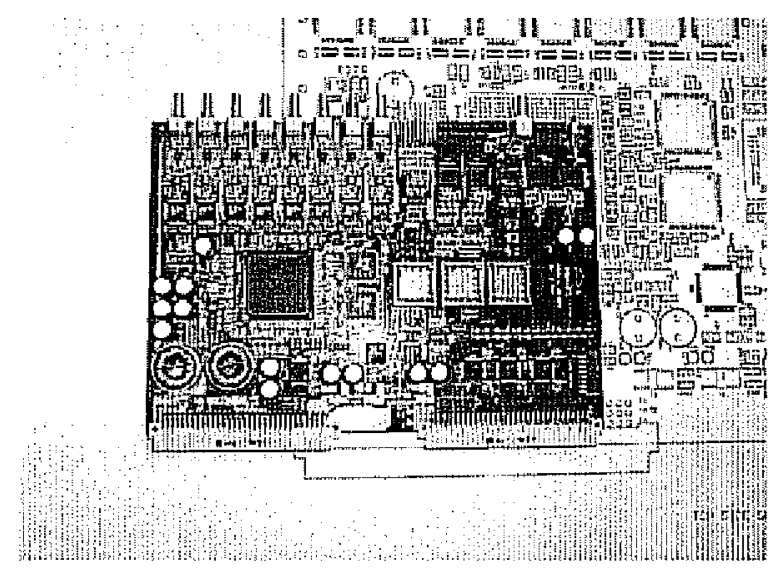

Figure 1: The prototype digital signal processing board has eight pulse shape digitizer channels each with a 100 MHz 12-bit ADC. Data are processed on board using a Virtex II FPGA and read out via a VME bus.

\section{Publications}

I.Y. Lee, "Status of GRETA (slide report)," AGATA Electronics and Data Acquisition Meeting, , submitted for publication, 9/19/02.

http://www,nsg.tsl,uu,se/agata/padova-sep2002/

\section{Detector Research and Development for Low Energy Solar Neutrino Detectors}

Principal Investigators: Kevin Lesko

Project No.: 02047 made for the first experimental evidence for double beta decay. With the evidence for neutrino mass and mixing mounting, the scientific case for additional experiments utilizing low solar neutrinos and additional more sensitive double beta decay experiments is increasingly strong. These experiments all share a common sensitivity to internal and external backgrounds encompassing both ubiquitous radioactive species and cosmic-ray generated species. The first part of this LDRD is aimed at studying these backgrounds and to investigate techniques for shielding or reducing them in next-generation experiments.

The primary reason for constructing solar neutrino, dark matter, double beta decay or low level counting experiments in a deep underground facility is to shield such detectors from the backgrounds induced by cosmic rays. When cosmic rays traversing the rock surrounding these detectors, they can create very energetic neutrons (with energies upwards of hundreds of $\mathrm{MeV}$ ) through spallation. It is possible for these neutrons to enter the sensitive regions of these experiments and fake the physics signals being sought for without triggering the veto counters that are sensitive primarily to charged particles. Another class of neutral-particle background originating from the rock, albeit with much lower energy $\sim 10 \mathrm{MeV}$, comes from $(\alpha, n \gamma)$ and $(\alpha, p \gamma)$ reactions in the rock. Typical rock and many construction materials have a ${ }^{238} U$ or ${ }^{232} T$ th chain concentration at the parts-per-million levels. Because of the large rock volume, the flux of fast neutrons and $\gamma$ 's emerging from the rock surface of the cavity can be a significant source of background to these experiments. These radiative $\alpha$-capture reactions occur not only in the rock, but also inside the construction material of the detectors due to the presence of minute amount of ${ }^{238} U$ or ${ }^{232} T$ Th. Decays of the daughters in the ${ }^{238} U$ or ${ }^{232} T h$ chains emit $\alpha$ 's, $\beta$ 's and $\gamma$ 's, which must also be included in estimating the background for these experiments.

In the design of experiments with very low count rates, shielding of the aforementioned backgrounds is of utmost importance. We have been developing a framework to understand these backgrounds at different depths underground and the strategies for shielding these backgrounds in the next generation of low count rate experiments of astrophysical interests.

\section{Accomplishments}

Our framework for this project is built on GEANT, a popular modeling program for detector and physics simulations. There are reasons for choosing this package instead of building a customized framework. Unlike other widely available simulation packages [(e.g. electron gamma shower (EGS)], which focus on solving very specific 
problems, GEANT is a collection of libraries that allows a wide range of particle interactions at different energy scales to be simulated. Because it is developed, distributed and maintained by the European Laboratory for Particle Physics near Geneva, Switzerland (CERN), adequate user support (e.g. bug fixes) is provided. In addition, GEANT is supported at the computing system at which this work is performed, the Parallel Distributed Systems Facility (PDSF) cluster maintained by the National Energy Research Scientific Computing (NERSC) Center. This allows us to concentrate on the physics study instead of cross platform compatibility issues.

In the past few months we have carried out a number of calculations at PDSF. These calculations mainly concern the problem of $\gamma$-ray shielding. Upon the GEANT framework, we studied the shielding requirements for a typical next generation low energy $p p$ solar neutrino experiment that uses neutrino-electron elastic scattering as the principal detection mechanism. An example of this type of experiment is the Helium Roton Observation of Neutrinos (HERON). In this experiment, the central sensitive region is a cryogenic liquid (superfluid Helium), surrounded by layers of moderators for background shielding and temperature control. The large amount $s$ of material in the cryostat is of particular concern in generating these backgrounds.

Using the PDSF cluster, we have been able to determine with high statistics the $\gamma$-ray backgrounds from different sources. The Figure shows the fraction of $\gamma$-rays originating from the rock cavity that penetrate into HERON for $\mathrm{E}_{\gamma}=2.6$ $\mathrm{MeV}$ and $8.6 \mathrm{MeV}$, which are respectively the typical $\gamma$-ray energies for decays of ${ }^{298} \mathrm{U}$ or ${ }^{232}$ Th daughters and radiative $\alpha$-capture reactions. The results of this study were compared to a low statistics study performed by the HERON collaboration, and the consistency between the two studies has been demonstrated. Our study also provided a baseline for the required cleanliness of construction materials in this experiment.

We have recently embarked on a more ambitious program to determine the total neutron backgrounds due to muon spallation in the rock and from the radiative $\alpha$-capture reactions. This will be achieved by building upon the work mentioned under the general GEAN'T framework.
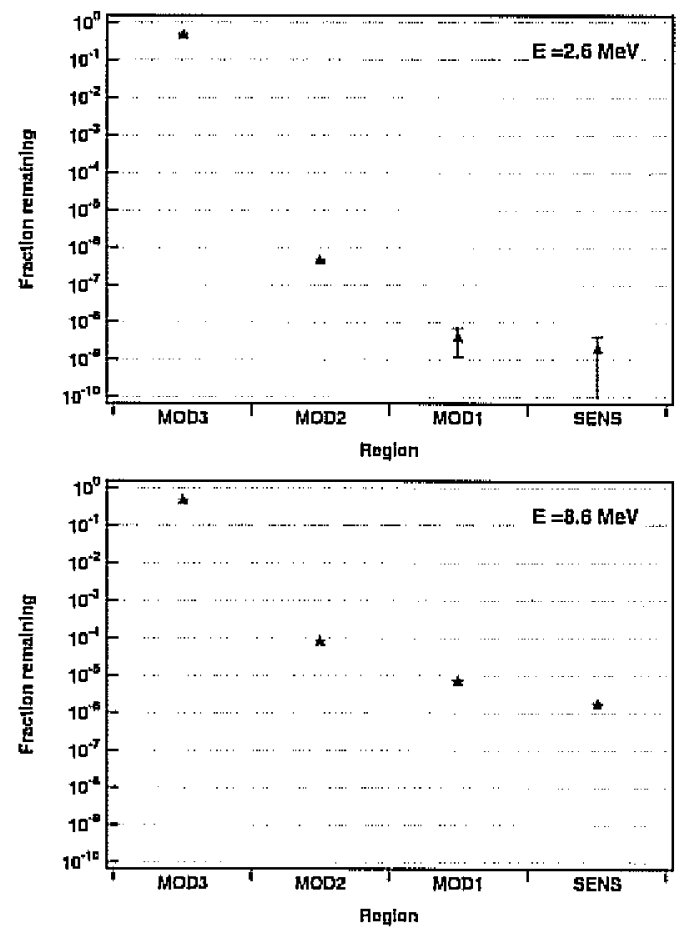

Figure 2: Calculation of the fractional leakage of background $\gamma$-ray from the rock cavity into a proposed solar nettrino detector (HERON). The labels in the abscissa represent different shielding regions (MODX) and the sensitive region (SENS) in the detector. 


\section{Research on a Next Generation Vertex Detector}

Principal Investigators: Howard Wieman, Fred Bieser, Stuart Kleinfelder, and Howard Matis

Project No.: 02048

\section{Project Description}

This project will develop an improved vertex detector technology suitable for the next generation experiments at Relativistic Heavy Ion Collider, Brookhaven National Laboratory (RHIC), heavy ions at Large Hadron Collider, CERN (LHC) and Next Linear Collider (NLC). There is tremendous interest in high energy heavy ion physics, including the Solenoidal Tracker at RHIC (STAR), PHENIX, and PHOBOS collaborations) in installing new thin, high-resolution vertex detectors to measure open charm through detection of D mesons. We propose development work on a recently demonstrated technology, Active Pixel Sensors (APS) in standard complementary metal-oxide semiconductor (CMOS), which could be the most effective solution for meeting the vertex detector requirements at future high-energy colliders. There are several demanding technical issues, electronic and mechanical, that must be solved before practical detectors can be made using this new technology.

We start with the recent demonstration that minimum ionizing particles can be detected with APS constructed in standard CMOS technology. We will address a number of design issues in order to move this promising demonstration of APS into a practical detector system design. Methods for on-detector-chip data zero suppression and readout will be developed. Issues of mechanical support and electronic interconnection of multiple chips into a detector structure will be investigated. The appealing uspect of this CMOS approach is the opportunity to combine detector and readout functions on a single chip using the latest readily available commercial IC technology.

\section{Accomplishments}

Using MOSIS (a service for integrated circuit prototype and fabrication), we have produced two APS chip designs with the 0.25 micron standard CMOS process of TSMC (Taiwan Semiconductor Manufacturing Company, Ltd). Tests with $\mathrm{Fe}^{55} \mathrm{X}$-rays and the $1.5 \mathrm{GeV} / \mathrm{c}$ electron beam at the Advanced Light Source (ALS) confirm the results of the original work by LEPSI/ReS (Laboratoire d'Electronique et de Physiques de Systemes Instrumentaux, Strasbourg) with other CMOS processes showing that these devices provide good signal to noise with minimum ionizing particles. Some results from these tests:

- The noise per pixel after correlated double sample (CDF) signal processing is 17 electrons rms.

- The most probable signal amplitude with minimum ionizing particles is 450 electrons.

- The signal amplitude is consistent with charge collection from the underlying 8 micron epi tayer.

- The charge loss due to recombination before signal collection is not significant.

- The measured leakage current is $0.9 \mathrm{fA}-1.6 \mathrm{fA}$ per pixel. This adds a background component that must be subtracted, but the associated shot noise will not be significant for our charge integration times between frame reads.

- A measure of the diffusion pattern of the signal in the epi layer shows that $89 \%$ of the signal from a point ionization in the epi layer is collected in the 5 by 5 array of pixel nodes centered on the point.

Comparison of these results with anticipated detector requirements of $98 \%$ detection efficiency for min-I particles and accidental hit tolerance of less than $100 \mathrm{hits} / \mathrm{cm}^{2}$ show that detectors with slightly better signal to noise are required. The currently available and demonstrated options are CMOS processes with thicker epi layers. Improved charge collection, i.e. reduced spreading of the signal to multiple nodes is still a possible option using the photo-gate technology, but this remains to be demonstrated in practice.

We have measured the radiation tolerance of our devices using $55 \mathrm{MeV}$ protons at the 88 " cyclotron. The findings from this study are:

- The basic CMOS operation shows no degradation with our maximum fluence of $1013 \mathrm{p} / \mathrm{cm} 2$.

- The radiation exposure reduces the signal by $\sim 40 \%$ for 1012protons $/ \mathrm{cm} 2$ (140 kRad). The implied lose mechanism is bulk damage in the epi layer which creates trapping centers.

- The radiation exposure increases the leakage current by $\sim 1 \mathrm{fA}$ for 1012 protons $/ \mathrm{cm} 2$ (140 kRad). This is a source of shot noise, but this will not be the limiting factor. 
- An estimate of the life time as a detector in the RHC radiation environment is 30 years. This is based on the predicted RHIC luminosity and the NIEL (Non Ionizing Energy Loss) scaling. The estimated accumulated dose in the 30 year life time is $140 \mathrm{kRads}$. This is for RIIC operation at 4 times design luminosity.

We have continued to refine simulations to better quantify the limits and benefits of an APS vertex detector for detecting $\mathrm{D}$ mesons. The results of this effort show that a detector of our proposed design would reduce the data collection time required for useful statistics by a factor of 50. The simulations continue to show significant benefits for reducing detector and beam pipe mass.

We have made good progress on a mechanical conceptual design that addresses issues of ultra thin detector support, cabling and cooling and rapid detector installation and replacement. Structures have been built to test the concept of using thinned silicon ladders. Silicon ladders have been thinned and polished to 50 microns and support and handling tests are being done. Our initial but very preliminary results show that these thinned silicon pieces are quite robust and significantly easier to handle than we had expected.

\section{Publications}

S. Kleinfelder, "Integrated X-ray and Charged Particle Active Pixel CMOS Sensor Arrays Using an Epitaxial Silicon Sensitive Region," Proceedings of the SPIE Hard $X$-Ray and Gamma Ray Detector Physics IV, July 2002. http://www.ece.uci.edu/-stuartk/SPIE2002_xray_talk.pdf

H.S. Matis, F. Bieser, S. Kleinfelder, G. Rai, F. Retiere, H. Ritter, K. Singh, S.E. Wurzel, and H. Wieman, "A CMOS Active Pixel Sensor for Charged Particle Detection," 2002 IEEE Nuclear Science Symposium and Medical Imaging Conference, Conference Record Archive, 2002, accepted for publicution.

H.S. Matis, F. Bieser, S. Kleinfelder, G. Rai, F. Retiere, H. Ritter, K. Singh, S.E. Wurzel, and H. Wieman, "Charged Particle Detection using a CMOS Active Pixel Sensor," IEEE Transactions On Nuclear Science (TNS), 2002, submitted for publication. http://macmatis.lbl,gov/LEEE_procedings_v2.doc 


\section{Physical Biosciences Division}

\section{Cooperative Effects Determining Fidelity in Cellular Recognition}

Principal Investigators: Jay Groves and Arup

Chakraborty

Project No.: 01035

\section{Project Description}

Cells interact with each other and their environment through myriald membrane-associated receptors and signaling molecules. Dynamic spatial reorganization of membrane receptors is a critical aspect of recognition and signal transduction mechanisms in a number of systems. Activation of T-lymphocytes is a prominent example. Initiation of the immune response requires large-scale, spatial self-assembly of T-cell receptors (TCR) engaged with major histocompatibility protein (MHC)-peptide complexes on an antigen presenting cell (APC). Formation of such an immunological synapse triggers an immune response. Although the cooperative reorganization of membrane proteins leading to the formation of functional immunological synapses has recently been observed, a physical understanding of the forces driving their organization remains elusive. We plan to mount a quantitative investigation of the physical characteristics and principles governing molecular reorganization events during initial stages of cellular recognition and signaling. Elucidating these principles may also reveal unexpected drug targets as well as biologically inspired strategies for recognition and patterning useful for materials design.

Our approach is aimed toward elucidating how physicochemical parameters determine the formation of spatiotemporal patterns. This will allow us to delineate the conditions that lead to the formation of signaling junctions. An investigative platform that combines novel membrane experiments and synthetic biochemistry with sophisticated theoretical calculations and computer simulations has been formulated to meet our goals.

\section{Accomplishments}

\section{Synaptic Pattern Formation}

As discussed in last year's report, the membrane introduces a fluctuating two-dimensional reaction environment in which topographical constraints and restricted component access strongly influence receptor binding and spatial organization. Our model sheds light on the observed sensitivity of the immune response to specific MHC-peptide mutations. Preliminary studies with the model we have developed also suggest a possible reason for the differential morphology of T-cell and NK-cell synapses based on protein binding kinetics. Another salient characteristic of the NK-cell synapse is that it forms without need for any active cytoskeletal intervention, and this underscores the hypothesis that synapses form by self-organization processes.

\section{Experimental Systems}

Provocative results emerging from our recent theoretical studies create an urgent need for experimental systems, which can help bridge between in vivo observations and the computational analyses mentioned above. As a first step along this path, we have initiated development of a totally reconstituted inter-membrane junction consisting of a supported membrane onto which a second membrane is deposited by rupture of a giant vesicle. Both membranes exhibit lateral fluidity, thus enabling molecular reorganization processes such as those that occur in the junction between living cells. This system recreates the environment of a cell-cell junction and will serve as a platform for our reconstitution experiments.

Initial studies of the immune synapse suggest that mechanical bending of the membrane drives protein sorting. Correspondingly, topographical patterns in the membrane reflect the underlying protein organization. The ability to image these patterns is critical to understanding reaction mechanisms in the synapse. We have recently introduced two strategies for imaging nanometer-scale topographical features in reconstituted membrane junctions. The first is based on intermembrane fluorescence resonance energy transfer (FRET). Our studies of glycolipids and proteins with well-known structures have demonstrated that intermembrane FRET can resolve membrane spacing with Angstrom resolution. We have used this to resolve spontaneously forming patterns in the reconstituted system. Significantly, the patterns visible in the polyethylene glycol (PEG)-lipid junction are caused by the same mechanism (membrane bending energetics) that our model predicts drive synaptic pattern formation in living T-cells. 
The second topographical imaging strategy is based on optical standing wave interferometry. This enables real-time mapping of the membrane surface with nanometer precision and provides resolution extending hundreds of nanometers from the surface. Using this system, we have been able to directly observe membrane thermal fluctuations, which create an entirely new set of opportunities for the study of molecular events at intermembrane junctions. Fluctuation amplitudes are exquisitely sensitive to forces that couple the membrane together. Reduced amplitude thermal

fluctuations indicate binding interactions between the two membranes. The strategy is unique in that it resolves binding events in addition to spatial localization. This process, which can be thought of as a form of "molecular sonar", provides us with a new and powerful window into the dynamical properties of ligand receptor engagement at intermembrane junctions.
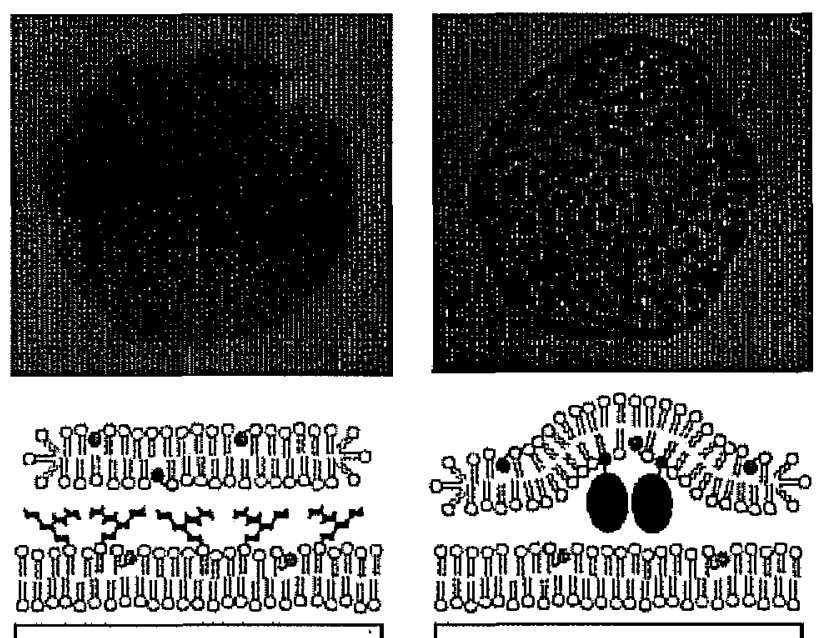

Subistratu

Substrate

Figure 1: Membrane bending induced pattern formation in a reconstituted intemembrane junction. Images are obtained using a novel intermembrane fluorescence resonance energy transfer technique that provides nanometer scale topographical resolution of the interface.

\section{Publications}

A.P. Wong and J.T. Groves, "Molecular Topography Imaging by Intermembrane Fluorescence Resonance Energy Transfer," Proc. Natl Acad. Sci. USA, 99, 22, 14147-14152, 2002.

S.J. Lee, Y. Hori, J.T. Groves, M.L. Dustin, and A.K. Chakraborty, "Correlation of a Dynamic Model for Immunological Synapse Formation with Effector Functions:
Two Pathways to Synapse Formation," TRENDS linmunology, 23, 10, 492-499, 2002.

S.J. Lee, Y. Hori, J.T. Groves, M.L. Dustin, and A.K. Chakraborty, "The Synapse Assembly Model," TRENDS Immlunology, 23, 10, 500-502, 2002.

T.R. Weikl, J.T, Groves, and R. Lipowsky, "Pattern Formation During Adhesion of Multicomponent Membranes," Eturopltys. Lett, 59, 6, 916-922, 2002.

A.P. Wong and J.T. Groves, "Topographical Imaging of an Intermembrane Junction by Combined Fluorescence Interference and Energy Transfer Microscopies," J. Am. Chem. So. 123, 12414-12415, 2001.

S.Y. Qi, Jay T. Groves, and Arup Chakraborty, "Synaptic Pattern Formation During Cellular Recognition," Proc. Nat'l Acad. Sci. USA, 98, 12, 6548-6553, 2001.

\section{Molecular Recognition and Protein/Protein Interactions in Signal Transduction}

Principal Investigators: Thomas Earnest; Randy Moon (HHMI, U. of Washington)

Project No.: 01034

\section{Project Description}

The interactions of proteins with other proteins, ribonucleic acid (RNA), or deoxyribonucleic acid (DNA) give rise to a great number of molecular and cellular functions. As genomic sequencing projects produce large numbers of samples, the need to characterize these samples in terms of their function becomes of great importance. We will use members of the Wnt signaling pathway-important in development and cancer-to explore interaction partners using molecular and cellular biology, along with synchrotron-based biological crystallography of the molecular complexes. We will also initiate strategies and methods to automate the exploration, expression, and structural studies of functionally interacting biomolecules.

In particular, we will use two-hybrid analysis to systematically investigate and identify the interactions of proteins in the Wnt pathway with other proteins or sets of proteins. Once these interacting partners are identified, coexpression in a variety of expression systems, followed by purification, crystallization, and structure determination of 
the complexes will proceed. Three-hybrid screens will be attempted once binary complexes are identified. The ability to discover, express, and crystallize higher-order complexes will be pursued for structural studies at the Berkeley Center for Structural Biology (BCSB) at the Advanced Light Source. Verification of interactions will proceed by molecular biological, biochemical, structural, and cellular methods. Development of robotic methods and approaches for high-throughput protein-protein interaction studies will be a high priority.

\section{Accomplishments}

Expression of proteins and protein complexes can be optimized by parallel insertion into numerous expression. vectors in E. coli and baculovirus-infected insect cell lines. We have developed a procedure for parallelizing attempts to achieve higher levels of expression by making several constructs with differing lengths and end residues on the $\mathrm{N}$ and C-terminals, utilizing green fluorescent protein as a fusion protein at the C-terminal of the protein of interest as a marker for expression and solubility. This method has been used to screen constructs (tagged arid untagged) to more rapidly determine which constructs (or pairs of binding-partner proteins) are more likely to be successful when used for purification, crystallization, and structure determination.

When a full-length protein or protein complexes do not express well it is often necessary to try expressing the domains which make up the proteins as separate constructs. This is a time consuming process since constructs that are very stmilar, differing in length by only one to ten amino acids at either end, can behave very differently. It is important to explore multiple parameters to increase the chances of finding constructs that express well. We are working with a system that combines two main ideas, recombination based cloning rather than restriction enzyme based cloning and the utility of C-terminal green fluorescent protein (GFP) tags to monitor folding to allow us to rapidly create and assay multiple constructs. The recombination based cloning system utilizes the Gateway system (Invitrogen, Carlsbad, California). A multifunctional vector, $\mathrm{pDEST}-\mathrm{HGH}$ has been developed, for use with the Gateway system. Constructs which result from the insertion of cDNA into this vector contain an $\mathrm{N}$ terminal His-tag and a C-terminal GFP-His tag. Our constructs insert a hexa-histidine tagged, and stop codon in the polymerase chain reaction (PCR) step. The LE392(DE3) cell line contains tRNA's which will read through this stop codon. In a "normal" cell line this vector will produce protein with an $\mathrm{N}$-terminal His-tag while in the LE392(DE3) cell line this construct will produce a mixture of protein with an N-terminal His-tag and protein with an
N-terminal His-tag and a C-terminal GFP-His tag. The intense fluorescence from the GFP allows the percentage with the C-terminal GFP-His tag to be assayed by pelleting the cells and exposing them to ultra violet (UV) light.

Using a test case which illustrates the utility of this system Drosophila Groucho, a protein that has five domains has been investigated. Using the described domain boundaries and hydropathy plots as guides we choose 3 forward primers for each of the domains. There are 4 reverse primers for the C-terminal domain, no reverse primers for the $\mathrm{N}$-terminal domain and 3 reverse primers for the internal 3 domains. Nine of the twenty-nine constructs did not have any fluorescence under a UV lamp and the remaining 20 did fluoresce under UV excitation. All of the proteins which did fluoresce gave high yields of soluble protein. Other members of the Wnt pathway (CtBP, CBP/beta-catenin) have been extensively subjected to twohybrid screening and coexpression, purification, crystallization trials and the correlation of fluoresence with over-expression of soluble protein has been high.

We have developed this methodology with a focus toward high-throughput production of proteins and protein complexes and recently taken steps toward automating and parallelizing this process.

\section{Publications}

B.N.R. Cheyette, J.S. Waxman, J.R., Miller, N. Khlebtsova, K.I. Takemaru, L.C. Sheldahl, J. Webster, E.P. Fox, N.J. Biery, J.D. Brown, T.N. Earnest, and R.T. Moon, "Dapper, a Dishevelled-Associated Antigonist of the Wnt//-catenin Signaling Pathway, is Required for Notochord and Head Formation, Developmental Cell 2, 449-461, April 2002.

N. Friedland, L. Hung, B. Cheyette, J. Miller, R. Moon, T. Earnest, "Conformational Flexibility in the PDZ Domain of Dishevelled Induced by Target Binding," in preparation. 


\section{Teraflop Challenges in Single-Particle Electron Crystallography}

Principal Investigators: Robert Glaeser, Kenneth
Downing, Eva Nogales, Esmond $\mathrm{Ng}$ and Ravi Malladi

Project No.: 00017

\section{Project Description}

The goal of this project is to define efficient mathematical approaches (and algorithms) that produce a threedimensional reconstruction from high resolution, cryoelectron microscopy (EM) images of single protein molecules. These tools must make it possible to automatically identify between $10^{5}$ and $10^{6}$ single particles and automatically merge these images to produce the threedimensional reconstruction. In order for this to be practical, identification and merging should be accomplished with less than $-10^{17}$ floating point operations $\left(\sim 10^{5}\right.$ teraflop). The long-term goal is to be able to carry out structural studies of large, multi-subunit protein complexes at high resolution, using electron microscope images of fields that contain -100 particles each. Merging data from single particles is equivalent to crystallization in silico. By eliminating the need for biochemical crystallization, and by reducing data collection and three-dimensional reconstruction to about one day each, single-particle electron crystallography will achieve a level of high throughput that is similar to the speed of $x$-ray crystallography. There will be no delay in screening for crystallization conditions, however. Furthermore, structural studies will be made possible for complex molecular assemblies that represent increasingly problematic challenges for $x$-ray crystallography, but which are increasingly more favorable for cryo-electron microscopy.

To accomplish this, existing methods of automatic particle identification will be refined by the addition of conceptually new mathematical tools. The goal is to improve particle identification to a level such that false negatives fall below $25 \%$ and false positives fall below $10 \%$. In parallel with this effort, we will port existing software suites (SPIDER, EMAN and others) to the Alvarez Linux cluster and the SP supercomputer at the National Energy Research Scientific Computing (NERSC) Center, and we will use existing data sets (one with almost $10^{5}$ particles) to demonstrate that routine, high-throughput merging of very large data sets is feasible on this machine.

\section{Accomplishments}

Work has continued during this past year on the problem of closing off particle contours between two particles that are quite close to one another. One of our proposals, i.e., to take into consideration the sign of the gradient at the edge, has proven to be less effective than expected. Effort has therefore concentrated on using modified stopping criteria for the "shrink wrap" contouring approach, and work in this direction is continuing. These efforts have been augmented by the investigation of traditionally used cross-correlation (matched filter) setrches, applied to images that were smoothed with the edge-preserving algorithm. A paper describing this latter work has been submitted for publication.

Parallelized versions of the portions of the SPIDER code that represent the most time-consuming steps in singleparticle data processing have been developed with the message-passing interface (MPI) standard. These modules have now been incorporated into a user-version of the SPIDER software package that runs on the large "Alvarez" Linux cluster at Berkeley Lab. A separate, parallelized version of SPDER is also running on the NERSC Center IBM SP machine (Seaborg), and this version has been used to gain experience with ideas that can ultimately be used on teraflop or higher machines. New theoretical work on matrix-free construction of preconditioners together with numerical testing has been applied to problems of 3-D reconstruction in single-particle data processing, and a paper submitted on this work is now in press.

A revised National Institutes of Health (NIH) Program Project grant proposal was submitted to NIH for the Feb. $1_{\text {. }}$ 2002 deadline. This proposal was based upon the preliminary results obtained during the work that was completed with this LDRD funding. The proposal has received a favorable review, and we hope that it will be funded by NIH in spring 2003.

\section{Publications}

W.V. Nicholson, R. Malladi, "Correlation-based Methods of Automatic Particle Detection in Electron Microscopy Images with Smoothing by Anisotropic Diffusion," Journal of Microscopy, submitted for publication.

C. Yang, E.G. Ng, P.A. Penczek, "Matrix-free Constructions of Circulant and Block-circulant Preconditioners," Journal on Nunerical Linear Algebra with Applications, in press. 


\section{Novel Synchrotron Experiments to Determine Hydration Forces for Molten Globules and Model Proteins for Extremophiles}

\author{
Principal Investigators: Teresa Head-Gordon
}

Project No.: 01036

\section{Project Description}

The aim of this proposal is to advance the transferability of our solution-scattering/simulation technique to new areas of biological interest, namely that of molten globule proteins, and hydrated, model protein systems that are explored at extremes of pressure and temperature. These new experiments will allow us to address two important biological systems. First is the structural organization of the hydrophobic core of molten globule proteins, a thermodynamic protein-folding intermediate that is thought to be a good model of kinetic intermediates for generic proteins. Second, the same techniques will allow us to explore the changes in physical hydration forces over large ranges in temperature and pressure, to address a primary research question in the area of extremophile organisms: how proteins necessary for life under these extreme conditions can remain stable and functional.

The experimental repertoire of protein-based structural techniques has resulted in a good understanding of a protein's secondary structure and tertiary structure contacts, while approaches to characterize the role of the hydration environment in terms of structure and forces in folding are comparatively minimal at present. We have combined our expertise in solution-scattering experiments, simulations, and theory, to define an approach to determine hydration structure and forces in protein solutions. We propose to perform novel selenium-edge and second-order difference, solution-scattering experiments at the Advanced Light Source using selenium-methionine derivatives of both amino acid monomers and the protein $\alpha$-lactalbumin that will reveal the structural organization of the hydrophobic core. We will also extend these same experiments to understand hydration at extremes of temperature and pressure.

\section{Accomplishments}

\section{Relevance for Molten Globule Studies}

At dilute concentration under ambient conditions we have extracted a $\mathrm{g}_{c}(r)$ for leucines in aqueous solvent from experimental intensities using wide angle scattering that, when converted to a potential of mean force, shows free energy of stabilization at contact and separated by a water layer. At higher concentrations of up to 2.0 molar (an insufficient amount of water for monomers to have their own hydration shell), the experiments and simulations indicate that water stabilizes mono-dispersed and small clusters of amino acids, as opposed to more complete segregation of the hydrophobic groups. This is surprising in that the system is very hydrophobic by protein sequence standards, and therefore supports our hypothesis that a collapsed but water impregnated core could well be transferable into the context of late stages of folding of polypeptide chains.

This year we have investigated the low resolution structural organization, as a function of concentration ( $0.05-2$ molar) of various hydrophilic and hydrophobic amino acids in aqueous solution, at $\mathrm{pH} 7$ and room temperature. Small angle $x$-ray scattering experiments were conducted at the APS/BioCat beamline to provide information about size, shape and interaction of the structural organization arising as the solute concentration increases, for $Q$ values ranging between 0.008 and 0.4 angstrom ${ }^{-1}$. Following the evolution of the size and shape analyzed from the small angle scattering as a function of concentration has allowed us to determine more definitively the long-ranged structure, if any, of these solutions. Preliminary analysis suggests an organization of a polydisperse core model, with dimensions on the order of 10 angstrom, i.e. small clusters (2-4 amino acids) with a modified aqueous solvent shell.

A recent study of $\alpha$-lactalbumin mutated all hydrophobic amino acids to leucine, and all molten globule signatures were found to be present in this "minimized core." The recovery of amino acid and water correlations from a combination of experiment and simulation is the information needed to describe differences in structural organization of the hydrophobic core between native and molten globule states of $\alpha$-lactalbumin. The simulated quantities described above will aid the extraction of the residue correlations in the core, and provide a structural interpretation to those experiments. We are working with David King on synthesis of the minimized core sequence of $\alpha$-lactalbumin using fully deuterated leucine for eventual study using neutron scattering, and synthesis of a possible new minimalist core using Se-Met to study by $x$-raly scattering.

\section{Pure Water Studies}

We have reported a new, high-quality $\mathrm{x}$-ray scattering experiment on pure ambient water using synchrotron beam line 7.3.3 at the Advanced Light Source at Berkeley Lab. We have written a Chemical Reviews article that describes the current status of the field regarding water structure determination by experiment and simulation. We have 
recently completed $\mathrm{x}$-ray experiments on neat water taken over a temperature range of $2^{\circ} \mathrm{C}$ to $77^{\circ} \mathrm{C}$ using a synchrotron beam line at the ALS. While the common procedure is to report both the intensity curve and radial distribution function(s), the proper extraction of the realspace pair correlation functions from the experimental scattering is very difficult due to uncertainty introduced in the experimental corrections, the proper weighting of $\mathrm{OO}$, $\mathrm{OH}$, and $\mathrm{HIH}$ contributions, and numerical problems of Fourier transforming truncated data in Q-space. Instead we consider the direct calculation of $x$-ray scattering spectra using ab initio density functional theory, and compare to our own theoretical analysis.

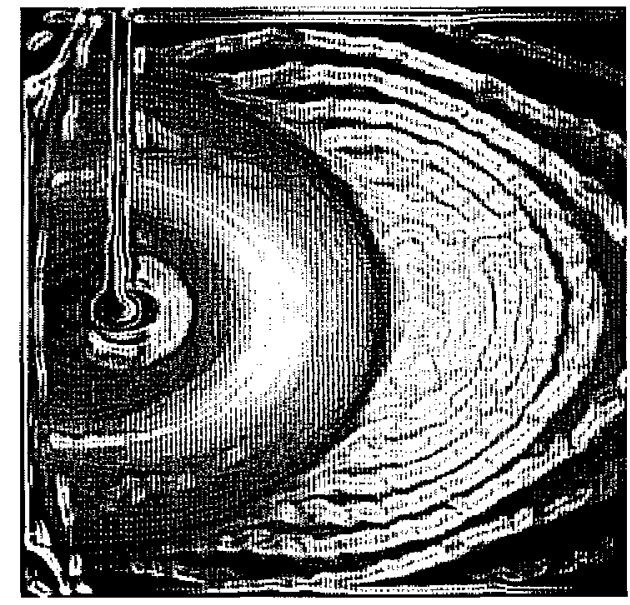

Figure 2: Detector image of synchrotron $x$-ray intensity data of room temperature water with film of liquid water over image.

\section{Publications}

G. Hura, D. Russo, R.M. Glaeser, M. Krack, M. Parrinello, and T. Head-Gordon, "Water Structure as a Function of Temperature From $X$-ray Scattering Experiments and Ab Initio Molecular Dynamics," Phys. Chent., 2003, in preparation.

T. Head-Gordon, G. Hura, J. Sorenson, R.M. Glaeser, "Pure Water Structure and Hydration Forces for Protein Folding, New Kinds of Phase Transitions: Transformations in Disordered Substances," Proc. NATO Advanced Research Workshop, Kliwer, Dordrecht, V8: 403-415, 2002.

T. Head-Gordon and G. Hura, "Water Structure from Scattering Experiments and Simulation," Chemical Reviews 102: 2651-2670, 2002 .

\section{Allosteric Mechanisms in Proteins Involved in Cell Signaling}

Principal Investigators: John Kuriyan

Project No.: 02040

\section{Project Description}

Crucial to the proper functioning of the cell is an elaborate control mechanism that interprets signals from the environment and then makes decisions controlling cell structure, transcription, and replication. My laboratory is studying the molecular mechanisms underlying these systems by focusing on the proteins involved in transducing signals generated by cell surface receptors. In the past this work has been driven primarily by $x$-ray crystallographic analysis of three-dimensional protein structures, a crucial first step in developing molecular mechanisms. This project is aimed at initiating studies in new directions that will combine biophysical analysis of protein function with the generation of mutations aimed at probing particular aspects of function.

The primary focus is on tyrosine kinases related to the protein coded by the Src oncogene. The members of the Src family of tyrosine kinases are controlled by tyrosine phosphorylation at two distinct sites that have opposing actions on the catalytic activity of the protein. Inadvertent activation of these proteins can result in uncontrolled cell growth and transformation of cellular phenotypes, the first steps towards cancer. We will use a semi-synthetic strategy to attach a fluorescent probe to the $\mathrm{C}$-terminal region of the Src kinase. The interaction between the C-terminal tail of the protein and the phosphotyrosine binding $\mathrm{SH} 2$ domain will be probed using fluorescence spectroscopy, as a function of the activation state of the kinase. This will provide information on the coupling between the $\mathrm{C}$-terminal region of the protein and the catalytic center, located more than 40 angstroms away. This information will be correlated with the results of molecular dynamics simulations and mutagenesis on the protein.

\section{Accomplishments}

We are currently focusing on two new and additional experimental approaches to the dynamics of protein kinase domains. First, we have initiated nuclear magnetic resonance (NMR) studies on protein kinase domains in collaboration with David Wemmer. The biggest challenge with these proteins, in terms of NMR studies, is to develop 
a bacterial expression system so that sufficient amounts of protein can be produced for isotopic labeling in a costeffective manner. Normally, most eukaryotic protein kinases are produced in insect cells, using a baculovirus expression system. Isotopic labeling is prohibitively expensive in this system. We have developed a bacterial expression system for two kinases, Calcium/calmodulindependent protein kinase-I (CamK-I) and p21-activated protein kinase (PAK) that are well suited for NMR studies. Initially, we are focusing on PAK. In this case we can produce approximately $50 \mathrm{mgs}$ of purified protein from about 1 liter of bacterial cell culture. Preliminary NMR spectra obtained in David Wemmer's laboratory (TROSY) show that it will be feasible to investigate the dynamics of this protein kinase by NMR.

Our second approach is to use yeast genetics to characterize the effects of mutations in the Src and Abl kinase domains, so as to map out the differences in the regulatory properties of these closely related kinases. This approach is being used to rapidly screen through large numbers of mutations in these proteins, with an aim of identifying those that will be interesting to study further in great depth. At the moment approximately 300 mutant forms of these kinases have been designed and generated, and they are awaiting phenotypic characterization in yeast.

Once results are obtained from these directions, we will return to the use of site specific fluorescent probes to study the kinetics of the activation process in protein kinases.

\section{Orphan Guanylate Cylase Receptors in C. Elegans}

\section{Principal Investigators: Michael Marletta}

Project No.: 02041

\section{Project Description}

Biological sensors occupy an essential biological niche in prokaryotic organisms to complex animals. Evolution builds in the required specificity and sensitively necessary for function. In some cases a molecular level understanding has emerged but we are far from fully appreciating the wide variations in sensor design.

Nitric oxide (NO) signaling is a particularly interesting example. Critical functions for this toxic, free-diffusible diatomic gas are now well established, including key role in cellular signaling and the host response to infection. In cellular signaling NO is biosynthesized by the constitutive isoforms of the enzyme nitric oxide synthase (NOS). Calcium and calmodulin (CaM) strictly control the activity of these enzymes. This activation is transient and highly regulated in order to avoid toxicity associated with the nonspecific chemical reactivity of NO. A highly specific and sensitive NO receptor is expected given the toxicity of $\mathrm{NO}$, and this is indeed the case in the form of the soluble isoform of guanylate cyclase (sGC). After activation by NO, this enzyme converts guanosine triphosphate (GTP) to cyclic guanosine monophosphate (cGMP) and pyrophosphate. cGMP subsequently brings about tissuespecific physiological responses such as blood vessel dilation via relaxation of vasetular smooth muscle. $5 G C$ is a heterodimeric protein composed of $\alpha$ - and $\beta$-subunits. The $\beta$-subunit contains a 5-coordinate, ferrous protoprophyrin IX heme that serves to bind NO. Once bound, $\mathrm{SGC}$ is activated about 400 -fold over basal activity.

Spectroscopic methods in combination with domain expression (residues $1-385$ of the $\beta$-subunit) and mutational analysis have been used to extensively characterize the heme environment in sGC. This environment has evolved to have several critical pieces that allow it to serve in the capacity noted above. Most notably, the heme in sGC does not bind oxygen. Since the concentration of $\mathrm{O}_{2}$ would dwarf that of $\mathrm{NO}$ in the target cell, this lack of binding of $\mathrm{O}_{2}$ is critical for NO to operate in an aerobic environment while using a porphyrin receptor. In addition, sGC and the heme domain construct bind NO with exceptionally fast on-rates, also permitting NO to function at very low cellular concentrations.

We have become interested in whether heme-binding cyclases exist in organisms like $C$. elegans. Our extensive characterization of the heme environment and catalytic activity has allowed us to predict similar heme binding motifs from genome sequence information. Sequence analyses of the $C$. elegans genome showed that it contains 7 $\beta$-type subunits. There are no predicted $\alpha$-subunits. This is a surprise given that all mammalian soluble cyclases characterized to date are $\alpha / \beta$ heterodimers. Even more interesting is that the sequence analyses place five of the $C$. elegans putative cyclases closest to the $\beta 2$-subunit. Homologues to the $C$. elegans putative cyclases can be found in $D$. melanogaster as well. The conclusion from our sequence analyses that the worm and fly putative $\beta 2$ subunit cyclases will bind heme.

In this project, we will continue to explore the nature of these predicted cyclases from $C$. elegans and attempt to answer long-term questions about the function of these proteins, extend our sequence comparisons to the globins in order to define the molecular determinates of oxygen 
sensors vs. NO sensors, truncate our $\beta 1(1-385)$ heme domain and attempt to solve the structure by nuclear magnetic resonance (NMR) and develop a construct to express $\beta 1(1-385)$ with $\alpha 1(1-400)$.

\section{Accomplishments}

\section{Cloning of the $\beta 2$ homologues}

We have cloned the five putative sGC's that are most closely related to the 32 -subunit $(g c y-32, g c y-34, g c y-35$, gcy-36, and gcy-37) by Reverse Transcriptase-Polymerase Chain Reaction (RT-PCR) and 5' and 3' RACE (Rapid Amplification of CONA ends). With the exception of the $5^{\prime}$ untranslated region (UT'R) for gcy-35, we have finished cloning these $\beta 2$ homologues. cDNA from a $C$. elegans mixed-stage cDNA library (Stratagene) and ribonucleic acid (RNA) from mixed-stage worms were used as templates.

\section{Neuronal localization}

We have completed the localization of $g c y-32, g c y-34$, gcy$35, g c y-36$, and $g c y-37$ are all localized to the candidate sensory neurons URX, AQR, PQR. gcy-35 also localizes to other neurons, possibly the well-studied touch receptor neurons.

\section{C. elegans heme domain constructs}

Based on our experience with a heme domain construct derived from the 81-subunit of rat SGC (residues 1-385), we have designed many gcy-35 constructs for expression in $E$. colli. The resulting studies were encouraging in that cell pellets from the cells containing the plasmid were red, suggesting the presence of heme. However, all the expressed protein was present in inclusion bodies. We have been unable to solve this problem, hence our interest in focusing on the rat $\beta 1(1-385)$ and sensor design.

\section{Knock-outs and genetic ablations}

Although the localization of the $\beta 2$ homologues to URX. $A Q R$, and $P Q R$ has provided some information about function, we would like to understand their role more completely. We have ablated URX, AQR and $P Q R$ so that we can determine the function of these relatively uncharacterized neurons. We are currently beginning to evaluate the phenotype of these worms.

\section{Truncated 1 1-(1-385)}

We are in the process of cloning truncated heme domains for NMR structure studies. All attempts to crystallize the 385 amino acid domain have failed. Preliminary evidence shows that a 260 residues fragment is stable and binds heme. We are working out the details of expression as well characterization of this new construct and will carry out some initial NMR experiments in collaboration with Dr. David Wemmer.

\section{Heterodimeric heme domain constructs}

The nature sensor is a heterodimeric protein. In an effort to more fully mimic the native enzyme, we have designed a polycistronic construct to co-express residues $1-385$ in the $\beta 1$-subunit and residues $1-400$ in the $\alpha 1$-subunit. We have just recently finished the construction of this plasmid and the protein is expressed. We are following up on this very exciting result. Characterization studies will commence soon and structural studies will be initiated in collaboration with D. John Kuriyan.

\section{Publications}

D.S. Karow, D. Pan, P. Pellicina, J. Kuriyan, R. Mathies, M.A. Marletta, January 30, 2003, in preparation.

D.S. Karow, J. Chang, J. Gay, C. Bargmann, M. A. Marletta, January 30, 2003, in preparation.

\section{Development of Techniques for Structural Analysis of Large, Multi- Subunit Eukaryotic Transcription Complexes}

Principal Investigators: Gerry McDermott

Project No:: 02042

\section{Project Description}

The path from gene to protein begins with transcription of deoxyribonucleic acid (DNA) to ribonucleic acid (RNA). In eukaryotes, transcription is performed by RNA Polymerase II (Pol II), a large multi-subunit macromolecular complex. Initiation and control of Pol II activity also requires up to forty additional proteins working in a concert. The majority of these proteins are involved in the formation of a PreInitiation Complex. Formition of the Pre-Initiation Complex begins when TBP (the TATA box binding protein) binds to a specific DNA sequence upstream of the gene to be transcribed. Once this occurs, a number of Transcription Factors (TFs) assemble, together with Pol H, to form a large protein-DNA complex. This complex is capable of basal levels of transcription. Complete regulation of transcription is governed by additional proteins, for example activators, that bind to specific DNA 
sequences, and interact with the Pre-Initiation Complex, in particular the multi-subunit complex TFID.

Structural information is now available on some components of the transcription machinery. Recently, the $X$-ray crystallographic structure of Pol $\Pi$ was determined to atomic resolution. However, clarifying a large body of experimental work, and understand transcription at the molecular level, requires high resolution structural information on TFWD, and other multi-subunit complexes.

The aim of this project is to develop techniques for the crystallization and structure determination of large multisubunit transcription complexes.

\section{Accomplishments}

This project began mid-year, and significant progress was made. Protocols have been established for over-expression and purfication of several individual subunits of the preinitiation complex, or their interacting partners. Our primary work has been to target proteins homologous to the TATA box binding protein, namely TRFI and TRF2. These proteins have been expressed at high levels in $\mathrm{E}$. coli and are now being subjected to initial crystallization trials, together with in vivo experiments such as gel mobility assays to determine optimal DNA construct for crystallization of protein-DNA complexes. We have also developed expression and purification protocols for the activator ADF1. This is currently being subject to crystallization trials in apo form, and as a complex with TFID subunit proteins.

\section{Conformation and Reaction Dynamics at the Single-Molecule Level}

\author{
Principal lnvestigators: Haw Yang
}

Project No.: 02049

\section{Project Description}

This research project seeks to develop single-molecule spectroscopic techniques for advancements in the design and application of single-molecule assays. The emerging picture of inferring biomolecule functionality from its 3dimensional structure is incomplete without a fundamental understanding of the microscopic dynamics. The capability of single-molecule spectroscopy offers a unique means to bridge the gap between the static structure and the dynamic functionality.

We will focus on developing single-molecule spectroscopic techniques to take the leadership in the design and application of single-molecule assays. The emerging picture of inferring biomolecule functionality from its 3-D structure is incomplete without a fundamental understanding of the microscopic dynamics. The capability of single-molecule spectroscopy offers a unique means to bridge the gap between the static structure and the dynamic functionality. It is the goal of this project to develop strategies that will allow, at the single-molecule level, a quantitative description of the dynamics and mechanisms that underlie a complex biological system.

\section{Accomplishments}

During the initial three-month period of 2002, we have successfully accomplished the design, construction, and initial characterization of a single-molecule (SM) spectrometer. The SM spectrometer was assembled to optimize the SM detection under various experimental conditions. Its modular design also allows for ease of maintenance and addition of detection units. Figure $3 \mathrm{~A}$ shows the spectrometer in operation.

A portion of the funding was directed to procurement of biochemical instrumentation and reagents that were necessary for sample preparations. Reaching this objective allowed us to prepare samples for characterization of the imaging aspect of the spectrometer. The experiments were carried out by raster-scanning a test sample, which was composed of single BSA-TMR (BSA, bovine serum albumin; TMR, tetramethylrhodamine dye) constructs immobilized on surface-activated coverslips. A representative image of such SM constructs is shown in Figure 3B. The full-width-half-maximum of a typical peak was approximately $300 \mathrm{~nm}$, as expected for diffractionlimited images. Also observed were other characteristics of single-molecule emissions, including step-wise photobleaching and intermittent blinking of the peaks. While optimization of the spectrometer continues, work on developing new single-enzyme assays is also underway. 

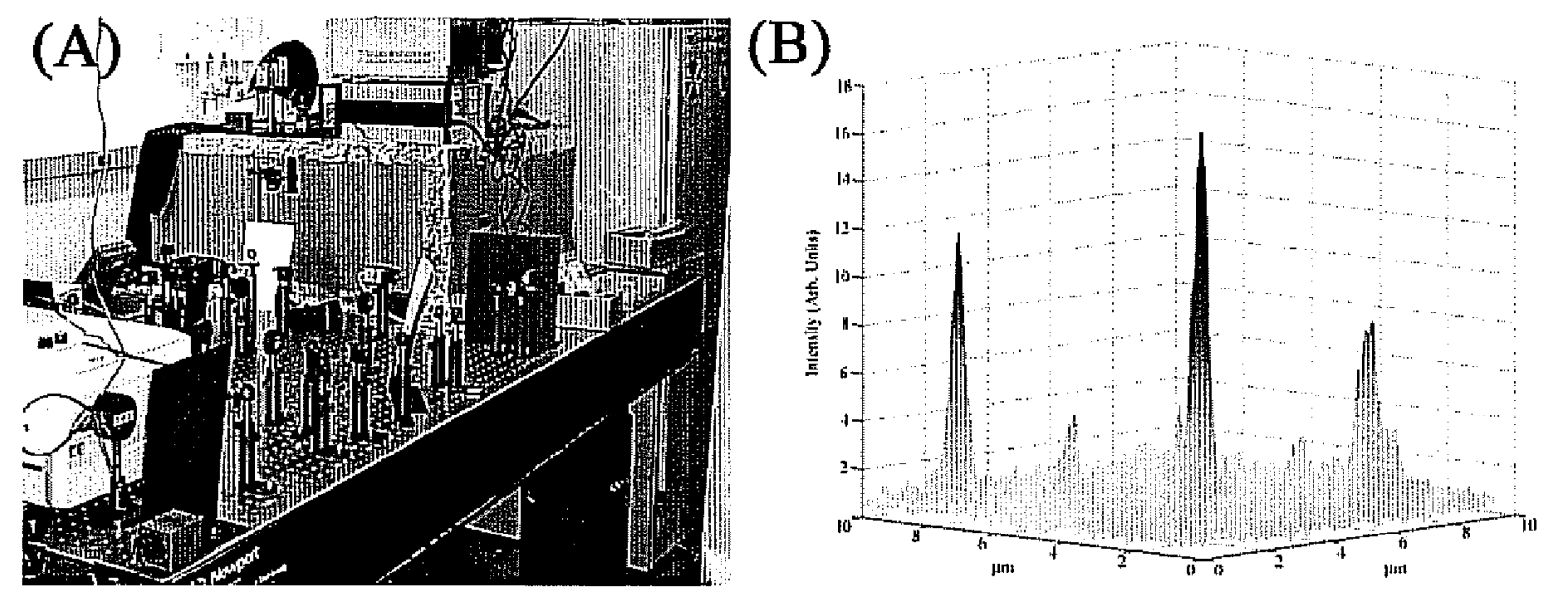

Figure 3: (A) The single-molecule spectrometer in operation. (B) A raster-scamed image of single tetramethylrhodamine (TMR) molecules immobilized on glass microscope coverslips. 


\section{Physics Division}

\section{Modeling of High Energy Physics Detectors}

Principal Investigators: Ian Hinchliffe and Murdock
Gilchriese

Project No:: 01037

\section{Project Description}

Monte Carlo simulation of detectors is a valuable tool for understanding the capabilities of the detector components and for planning strategies for using the detectors to reveal new physics processes. A crucial component of that will be the integration of the inner detectors into the ATLAS simulation and reconstruction. Two components of the inner detector, the pixels and silicon strip detectors, are being partially constructed at Berkeley Lab. A common framework describing the geometry of the system is essential. This will help us to understand how the inner detector can be used in the search for new physics signatures.

The new object oriented simulation tool, GEANT-4, has recently become available. Validation of the modeis underlying this tool is essential. This validation needs to take place well in advance of the tool's actual use. GEANT4 has important applications outside High Energy and Nuclear Physics including medical, accelerator, and space physics studies. This proposal will undertake a detailed comparison of the predictions of GEANT-4 with a system, the ATLAS pixel test beam, where data are available. This will enable validation and refinement of the underlying physics models in GEANT-4. Detailed comparisons of the predictions of GEANT -4 with actual data are required. Comparison will be made with test-beam data to be obtained at CERN in the future. A GEANT-4 model of the ATLAS pixel test beam will be made and will be integrated into the ATLAS software framework. Results from this will be used for a detailed validation of GEANT-4. The results will be used to adjust the underlying GEANT-4 model.

\section{Accomplishments}

During FY02, we used the Monte Carlo simulation of the detector to study the measurement of tau polarization in the
ATLAS detector. These early studies were carried out using the GEANT-3 simulation because the GEANT-4 program was not sufficiently developed and debugged. This work has led to the development of improved reconstruction algorithms. Work was completed that allows the integration of the GEANT-4 simulation package into the ATLAS framework. Work has now begun on designing and implementing new classes to describe the geometry of the detector. These classes will be used by both the GEANT-4 simulation and reconstruction. The first subsystem to be described is the silicon strips. Another important activity for FY02 has been the investigation of the impact of staging one of the pixel layers on physics signatures in the ATLAS detector.

The present focus of activity is on the use of GEANT-4 program that we will exercise as part of the next Data Challenge scheduled to begin in January 2003. We began to develop a description of the geometry of the Silicon strip and pixel detectors that will be used in this data challenge which will the first to use GEANT-4. We will use the data produced to test the GRID-based physics analysis model that we expect to be used for the real data in 2007.

The geometry description for the Pixel system in GEANT-4 was completed in October 2002. This provides a complete description of the materials in the Pixel detectors of the ATLAS detector in the form of GEANT-4 based C++ classes. This description was used as a prototype for testing. the implementation of the new GeoModel description of the complete ATLAS geometry from which the geometry for simulation and reconstruction will be obtained. It was demonstrated that the classes derived from this model could properly reproduce geometry of the GEANT-4 classes. Detailed comparisons of the material distributions have shown that this new description is at least us good as the old (FORTRAN) GEANT-3 system that ATLAS is currently using. A digitization scheme for the pixels was also produced and will be used as the basis for the digitization of the Silicon detector system. This GEANT-4 activity forms part of the first complete GEANT-4 description of the ATLAS detector that is expected to begin validation and testing on January 2003. This will enable ATLAS to retire the old FORTRAN based simulation and is a vital step in moving the entire offline code base to a modern object oriented design. 


\section{POLARBEAR: An Experiment to Measure Polarization Anisotropy in the Cosmic Microwave Background}

\author{
Principal Investigators: Adrian Lee and Helmuth \\ Spieler
}

Project No.: 01038

\section{Project Description}

This project will conduct research and development in support of a new experiment to measure Cosmic Microwave Background (CMB) polarization. The new experiment, called POLARization of the Background millimEter bAckground Radiation (POLARBEAR) combines the proven MAXIMA (Millimeter Anisotropy eXperiment Imaging Array) telescope and observation strategies with a new detector technology based on superconducting transition-edge sensors (TES).

The Cosmic Microwave Background is proving to be a peerless laboratory for cosmology and fundamental physics. Recently, measurements of the spatial temperature anisotropy by MAXIMA and BOOMERANG (Balloon Observations of Millimetric Extragalactic Radiation and Geophysics) have given strong support to inflationary models with a density close to critical. We have also been able to measure the density of baryons and cold dark matter, and the spectral index of the primordial power spectrum. Perhaps the most exciting promise of polarization measurements lies in theit ability to probe the inflationary epoch, i.e. as early as $10 \exp (-38)$ seconds after the Big Bang. Gravity waves produced at these early times propagate forward and produce structure in the photonbaryon fluid at the surface of last scattering, observable by its signature in the polarization of the CMB. Detection and characterization of this polarization will require a large leap in experimental capability. The key new technology for POLARBEAR is the large-format array of voltage-biased superconducting bolometers that are based on a superconducting film biased in mid-transition. Several tasks will be performed to examine the technical details of such a detector.

\section{Accomplishments}

We have continued the general design of and testing of components for the POLARBEAR receiver to arrive at a conceptual design for a full proposal. The main thrust areats have been the cryogenic system, the integration of antennas, bandpass filters and sensors, and the readout. These thrust areas are the same as for the previous year, but significant progress has been made.

POLARBEAR will use two refrigerators, a "pulse-tube" cryocooler to achieve an intermediate temperature of $2.5 \mathrm{~K}$, and a ${ }^{3} \mathrm{He} / 4 \mathrm{He}$ closed-cycle refrigerator to cool the detectors to $250 \mathrm{mK}$. The use of a cryocooler instead of liquid cryogens will facilitate the long integration times required for the success of this experiment. This year, we have fabricated a new test dewar that is a direct testbed for the final POLARBEAR dewar. The new 15" diameter dewar can house the pulse tube cooler and the $3 \mathrm{He} / 4 \mathrm{He}$ refrigerator intended for use in the final experiment. (Last year we were using a much smaller dewar that could not contain the final $3 \mathrm{He} / 4 \mathrm{He}$ refrigerator.) The new dewar will allow us to test the stability and noise properties of our bolometers under the same conditions as in the final experiment. The first cooldown of the new cryostat will be in December 2002.

We have just begun testing our first antenna-coupled bolometer prototype. Planar antennas with dual polarization (for coupling to the telescope), microwave band-defining filters, and bolometers are integrated onto a single substrate. The tests are just beginning, but the preliminary results look promising. We can see that the detectors are polarization-sensitive and that the noise properties are as expected.

We have been investigating the design of an economical Superconducting QUantum Interference Device (SQUID) readout for POLARBEAR. In the long term, we will use a SQUID-based readout multiplexer that we have originated. This multiplexer will enable arrays of several thousand elements. However, before we design and implement such a large array, we will deploy the receiver with a smaller array to test the detectors and observation strategy. The first step will be a -100 element array that we can build using a commercial SQUIDs and a custom set of SQUID readout electronics. We have built and tested a prototype of the custom room-temperature SQUID readout. Using this readout, we have now taken data with both single SQUIDs and a series array SQUID made by Seiko in Japan. We have explored the grounding and shielding requirements for the SQUID system. We have achieved adequate performance with both the single and array SQUIDs for the -100 element array. 


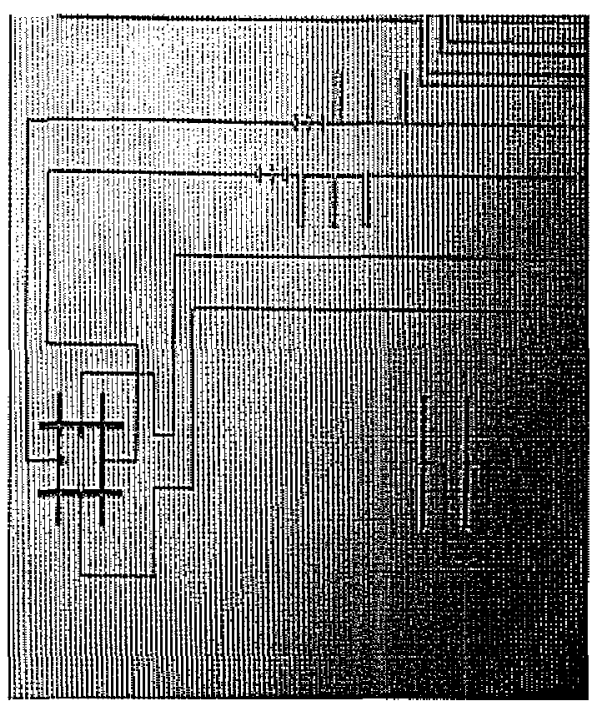

Figure 1: Photograph of prototype chip of antennacoupled bolometers. A $\mathrm{Nb}$ ground plane and $N \mathrm{~N}$ traces over oxide insulator are visible. For scale, the dipole antentas are ronghly $1 \mathrm{~mm}$ in length. The planar anteannas are double slot dipoles. Slot dipoles are simply slots cut in the ground plane, and are the Babinet complements of metal dipoles. The antennas accept radiation from the telescope, but suppress radiation from the surroundings. The radio frequency $(R F)$ energy from the antennas propagates along "microstrip" transmission lines, through frequency-band defining resonant filters, and finally is absorbed in the bolometer. The antenna to the left receives two orthogonal polarizations, and the signals from these antennas are differenced after the bolometers to form a differential polarimeter. This photo represents a new design compared to FYOI. This new design is currently undergoing test (see text for details).

\section{Publications}

A.T. Lee, H.M. Cho, J. Gildemeister, N.Halverson, W. Holzapfel, J. Mehl, M. Myers, T. Lanting, P. Richards, E. Rittweger, D. Schwan, H. Spieler, H. Tran, "Voltagebiased TES Bolometer for the Far-infrared to Millimeter Wavelength Range," Millimeter and Submillimeter Detectors for Astronomy, Proceedings of the SPIE, Kona, Hawaii (2002), submitted for publication. July 2002.

M. F. Cunningham, J. N. Ullom, T. Miyazaki, S. E. Labov, John Clarke, 'T. M. Lanting, Adrian T. Lee, P. L. Richards, Jongsoo Yoon, and H. Spieler, "High-resolution Operation of Frequency-multiplexed Transition-edge Photon Sensors," Applied Physics Letters, 81, Issue 1, pp. $1-184$.

\section{Foundations for a SuperNova/ Acceleration Probe (SNAP)}

Principal Investigators: Michael Levi, Saul

Perlmulter and Greg Aldering

Project No.: 00026 (\& 27)

\section{Project Description}

In FY00, Berkeley Lab funded two separate projects. Project 00026 is to tackle technical challenges in developing a large charge-coupled device (CCD)-mosaic camera having near-infrared (IR) sensitivity. Project 00027 is to develop techniques to significantly increase discovery of nearby supernovae-which is particularly constrained by data handling bottlenecks - for reducing the systematic uncertainties in our current understanding of supernova observations. Consequently, the two selfcontained reports follow.

For FY01, because both scientific problems are crucial to the possible development of the proposed Department of Energy/National Aeronautics and Space Administration SNAP satellite, they were considered effectively a single project. Nonetheless, both scientific problems are crucial for further general advances in astrophysics and astronomy and therefore are of ongoing significance independent of the ultimate success of the SNAP satellite proposal.

\section{Sub-Project 00026: Development of an Advanced Imaging Array}

\section{Project Description}

A major milestone has been achieved with the recent success in fabricating near-IR sensitive, large-format 2000 by 4000 pixel CCDs. We must now find ways to exploit this technology to the fullest. In particular, these devices can be placed in advanced cameras for observational astronomy and lead the new development of major scientific instruments for astrophysics research. We intend to develop a small-scale instrument, optimize it, and then use the demonstrated performance as a centerpiece for several new directions, which could include a major instrument for ground-based astronomy. 
For this project, we propose developing the capability to produce large CCD-mosaic cameras, from the design stage, to a complete scientific camera that can be used at a major telescope facility. The design would be applicable to many of the rext-generation ground-and space-based observatories. The currently available CCD camera designs are not as sophisticated-in their mechanical, electronics, readout, or computing designs-as the stateof-the art high energy physics detector systems. Thus, at Berkeley Lab, we are well suited to developing verylarge-area CCD arrays that can be used for very-deep high-resolution surveys of the early universe. The development would be in the context of creating a large instrument for a world-class wide-field telescope. This would allow us to aggressively pursue the uniqueness of our technology while exploiting the strengths of Berkeley Lab in creating large multi-faceted instruments.

\section{Accomplishments}

In previous years we have built the necessary clean room capabilities and have acquired liquid nitrogen Dewars needed to cool the CCDs. We have purchased two commercial CCD controllers and interfaced them to a Sun Workstation for testing. We have also built models of final production mounts that support backside illumination where a CCD is glued directly to a compatible metal. This scheme supports four-sided, close packing of the CCDs so that large-area arrays can be assembled.

The focus of the FY2002 activity was on the design of mechanical aspects of a camera composed of a 3 by 3 mosaic of large-format CCDs. Our commercial partner, DALSA, has produced excellent front-illuminated CCDs on $150 \mathrm{~mm}$ wafers. The major technical challenge is handling the $675 \mu \mathrm{m}$ after it has been thinned to 200-300 $\mu \mathrm{n}$ in preparation for polishing the backside. We have pursued three alternatives to address this issue. We have now developed a process for packaging CCDs so that we can carry out systematic measurements of their electrical characteristics (diffusion, interpixel response, cross-talk, uniformity of quantum efficiency, and radiation tolerance).

We have worked on a production capability to package devices with reliable connectivity. This must be done without electrostatic discharge (ESD) damage and without excessive mechanical stress on the CCDs. We have used the Berkeley Lab speckle interferometer to study detector distortion due to differential coefficient of thermal expansion of the CCD, the substrate and the mount. About a dozen configurations were tested and we have found an acceptable solution.

During the last year we have also been working on the implementation of the CCD mounting design to be used for general distribution of the devices to ground-based telescopes, in particular the new cameras under construction for the nearby Supernova Fuctory. We have carefully measured the mechanical deformation in the CCDs at the micron level as they are cooled to operating temperature. The deformation was due to the thermal mismatch of the silicon to the support material and the glued used to attach the two. We also continue to study the electro-optical performance impact of the strain induced in the CCD after cool down, again due to the thermally mismatched materials used to mount the CCDs. We have assembled the first devices that can be used for astronomical observations. We are also exploring the use of silicon as the CCD support material to eliminate all thermal mismatch issues.

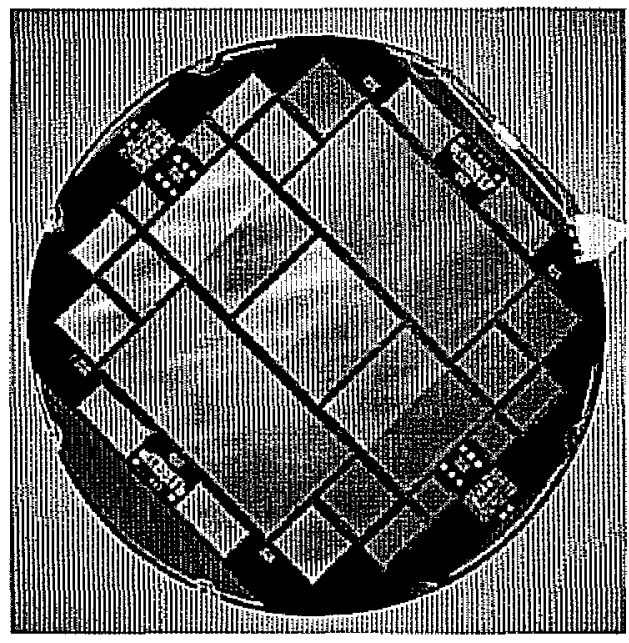

Figure 2: $150 \mathrm{~mm}$ DALSA-fabbed wafer. Large rectangular devices are $2 k \times 4 k, 15 \mu m$; large square devices are $2.8 k \times 2.8 k, 10.5 \mu \mathrm{m}$ with 4 -corner readout.

\section{Publications}

G. Aldering, for the SNAP Collaboration, "Overview of the SuperNova Acceleration Probe (SNAP)," Proceedings of the SPIE Conference on Astronomical Telescopes and Instrumentation, August 22-28, 2002, submitted for publication. http://snap.Ibl.gov/review/spie/SPIE_Overview.ps

M. Lampton, and C. Bebek, for the SNAP Collaboration, "The SNAP Focal Plane," proceedings of the SPIE Conference on Astronomical Telescopes and Instrumentation, August 22-28, 2002, submitted for publication. http:/snap.lbl.gov/review/spie/focalplane_revC.pdf 


\section{Sub-Project 00027: Nearby Supernova Search with Ten-Fold Increase in Efficiency}

\section{Project Description}

The study of cosmology has entered a new era in which empirical data can play a decisive role in determining the correct theory of the history and fate of the universe. While previously this theory was heavily dependent on esthetic considerations, we now are beginning to have a range of experimental /observational tools that can directly measure the relevant cosmological parameters. Among these tools, supernovae stand out as potentially the most direct, least model-dependent, for studying the energy-densities of the universe and the relative contributions of mass energy and vacuum (or "dark") energy. Over the past 15 years, Berkeley Lab has developed this supernova tool to the point that its current main result-the evidence for the existence of significant vacuum energy-is now considered to be a crucial element of the current State of the Universe report by much of the astrophysics community.

The next key step is to take the range of techniques that we have developed and perform the definitive measurements that will make the supernova results one of the solid foundations on which future cosmological investigations will butld. By studying an order-ofmagnitude more supernovae in a much larger, systematic program, we can address each of the main remaining sources of uncertainty (primarily systematics). The "supernova values" for the mass density, vacuum energy density, and curvature of the universe will then become the benchmarks for the other, more model-dependent cosmological measurement techniques. In particular, the cosmic microwave background measurements will be able to use these values as both a starting point and a benchmark as they fit the eleven or so parameters to which their power spectrum is sensitive.

This project will provide Berkeley Lab with the capability to dramatically scale up discovery of nearby supernovae. Instead of finding dozens of supernovae during a semester, we will gear up the search to discover several hundred and therefore are calling this a Supernova Factory (SNfactory). We are learning how to do this in an efficient manner, and learning which telescopes are most suitable for the task. In particular, the NEAT [Near Earth Asteroid Tracking program operated by the Jet Propulsion Laboratory (JPL)] search runs are going to increase. Over a three-month span, we would expect NEAT to find over 200 supernovae in the Hubble Flow.
We must greatly improve the computational tools for managing the dataflow, data analysis, scheduling, and multi-telescope coordination. We have identified the following items as critical tasks. They are all feasible, but will require innovative large-data-set handling, and novel software for coordination of an international collaboration of hurnans, computers, and telescopes. More complete automation of the search. Just controlling and monitoring the dataflow, required most of the time of two experienced scientist/ programmers during the period of the pilot study search. This will have to be automated for year-round searching. Automation of target sky-tield selection, and distribution to the appropriate telescopes (e.g., NEAT). We will need automation of the evaluation process of search observations and appropriate rescheduling.

We need to improve automated candidate pre-screening by at least a factor of 30 . We must develop more reliable network connections and use dedicated machines. We will need automation of follow-up observation scheduling for the multiple telescopes that will observe the supernovae after discovery. Automation is also needed of analysis of follow-up data feedback of these results for the planning and scheduling of further follow-up observations.

\section{Accomplishments}

There has been major technical progress in FY02. The wireless $45 \mathrm{Mbs}$ connection to Palomar that was installed in FY01 has been running for 475 days with only a few days with outages. This has permitted us to transfer images each night for analysis at Berkeley. The seurch pipeline is rurning regularly and almost unattended on Parallel Distributed Systems Facility (PDSF) computers at the National Energy Research Scientific Computing (NERSC) Center. So far we have archived 20 terrabytes of data and searched over $2 \times 10^{5}$ images. We have reported more than thirty-four Supernova candidates to International Astronomical Union Circulars. All twentyseven of the submissions with spectroscopy by others have been confirmed as SNe. We have 7 additional candidates which are probable SNe based on follow-up lightcurves. This is the best record ever for a first-year SN search.

In the instrumentation area there has also been important progress. For the Supernova Integral Field Spectrograph (SNIFS), optics fabrication has begun and proceeding on schedule. The first spectrograph CCD camera has been completed and is being shipped to our French collaborators. We have procured $C C D$ controllers and closed-cycle coolers for 2nd and 3rd CCD cameras. Berkeley Lab-produced $2 k \times 4 k$ CCD's have been packaged. The science grade CCDs needed for the redsensitive channel of SNIFS are expected soon. 
We have completed the prototype software for the supernova search so that we can now handle data from NEAT. During the second half of FY2002, we have evaluated a number of software systems developed for other astronomical projects. We have acquired OPUS (Operational Pipeline Unified Systems) and SPIKE from the Space Telescope Science Institute and Aspen and Casper software from the Jet Propulsion Laboratory. These programs manage the flow of data from telescopes and then schedule new observations. We determined that the scheduling aspect was the highest priority for us and we concluded that SPIKE was in the best state for us to use immediately. We have now got a version of SPIKE working with our data and it is generating schedules for a model telescope.

A Memorandum of Understanding (MOU) for use of University of Hawaii 2.2-m was completed November 2001. The MOU for use of Near Earth Asteroid Tracking data has also been finalized.

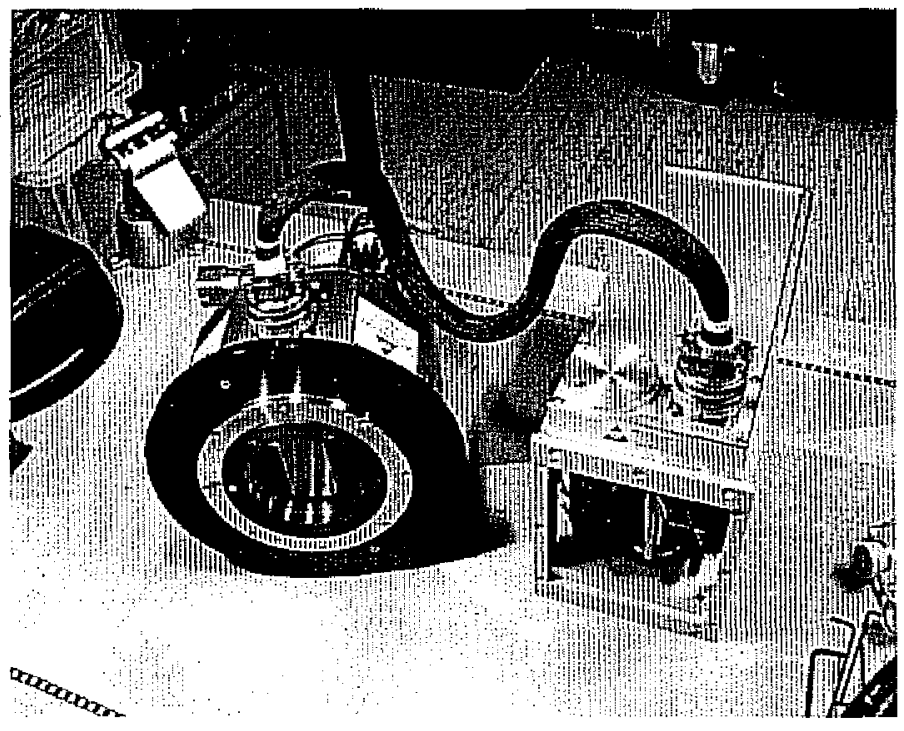

arch 2002, there was a Scientific Assessment Group for Experiments in Non-Accelerator Physics (SAGENAP) review of the project. This led to subsequent strong endorsement of the SNfactory by SAGENAP. In June 2002, there was a laboratory directors review in France. This resulted in a strong endorsement of the project's progress to date and application of further engineering effort to help maintain schedule for the commitments from France.

A major milestone for the project in FY02 was a detailed review of the SNAP R\&D plan by the Department of Energy. In this review, the proposed SNAP instrument suite was described and technical progress was presented. The design and testing of the CCDs and associated camera were a critical part of this review. The conclusion of the review panel was that the SNAP science case had been convincingly made and that the proposed instrument set was adequate to do the SNAP science mission. This means that the SNAP project is ready for Critical Decision $0(\mathrm{CD}-0)$ and can begin to receive project funding.

Figure 3: Bltu-sensitive Camera for the Stipernovi Integral Field Spectrograph (SNIFS) 


\section{Cross-Divisional}

\section{Computational Methods for Electronic Structure Codes in Materials Science (Ground-State and Excited-State Properties)}

\author{
Principal Investigators: Andrew Canning and Steven \\ Loule
}

Collaborator: Lin-Wang Wang

Project No.: 00007

\section{Project Description}

A fundamental understanding of the properties of a material requites the knowledge of its electronic structure as determined by the valence electrons. In particular as electronic components move towards the nanometer scale, quantum effects become increasingly important and can only be predicted and understood from accurate calculations of the electronic structure.

This project aims to develop state-of-the-art electronic structure methods and parallel codes and make them available to the materials science community and in particular the Department of Energy (DOE) community and National Energy Research Scientific Computing (NERSC) Center users. These codes will perform ground state as well as excited state electronic structure calculations. The ground state calculations are based on density functional theory (DFT) using pseudopotentials to represent the atomic core regions and the local density approximation (LDA) or the generalized gradient approximation (GGA) for the electron-electron interaction. The wavefunctions for the electrons are expanded in plane-waves. Codes for electronically excited states will also be developed using a first-principles approach developed by Louie and collaborators based on the GW approach (The "G" in GW refers to the dressed Green's function of the electron and the "W" is the screened coulomb interaction.) In the case of optical properties where an electron-hole pair (exciton) is formed the interaction between the excited electron and the hole is included through the two particle Green's function. The excited state codes require the ground state wavefunctions and energies as inputs so the complete set of codes is required for an excited state calculation.
Development of these codes and methods will be applications driven from physical problems relevant to the Materials Sciences Division (MSD) and Advanced Light Source (ALS) divisions at Berkeley Lab. These types of electronic structure calculations are very demanding of computer power and are a natural match for the large parallel computers at NERSC Center.

\section{Accomplishments}

\section{Ground State-Methods and Code}

In the density functional theory (DFT) code PARAllel Total Energy Code (PARATEC) i molecular dynamics scheme has been introduced into the code allowing ground state molecular dynamics simulations to be performed. The Grassman conjugate gradient method for metals has been further refined to include partially occupancy for the iterative diagonalization step. This is the first time an allbands conjugate gradient method has been formulated that uses partial occupancy for the iterative diagonalization step. This code has been used to interpret the results of Prof. Qiu's (UC Berkeley Lab) group for experimental studies of quantum well states in thin copper films grown on a cobalt substrate. These experiments were carried out at the ALS at Berkeley Lab. The new Grassman metal method was ctucial for converging some of the quantum well systems. More work has also been carried out on the web site (www.nersc.gov/projects/paratec) to add in more details about the performance of the code as well as including details of the new features of the code in the users guide.

\section{Excited State Methods and Codes}

The development of a method to calculate forces for excited states has been completed. We have developed a mathematical formalism to perform the calculations in the GW-BSE framework (The "G" in GW refers to the dressed Green's function of the electron and the " $W$ " is the screened coulomb interaction, BSE refers to the Bethe-Salpeter equation for the two-particle Green's function) and have implemented the method for parallel computers. The method has been tested on molecular systems (carbon monoxide and ammonia) and is as accurate as state of the art quantum chemical methods while scaling much better ( $\mathrm{N}^{4}$ instead of $\mathrm{N}^{6}$ or higher). The calculation of forces is as expensive as performing a single BSE calculation and is easy to perform with our new implementation. We are now studying the excited state relaxation of two systems: (i) selftrapping of excitons in silicon dioxide (alpha-quartz), and (ii) the cis-trans isomerization of the molecule azobenzene upon photoexcitation to the first excited state. 
We are now studying the optical properties of carbon nanotubes using our GW-BSE method. For metallic tubes, the screening of electron-hole pairs is significantly different from the standard semiconducting state. Therefore, we have modified the GW-BSE codes to correctly deal with the metallic screening and have fully tested our implementation. For the metallic tubes, the GW-BSE results for the optical spectra both agree well with experiments and also explain aspects of the spectrum that are not explicable using simpler interband models based on density functional theory. Unusually, certain metallic tubes display exciton binding. In semiconducting tubes, we find very strong quasiparticle and excitonic effects and are investigating the source of these effects.

Our GW-BSE codes have advanced to the point that other research groups are able to utilize them for scientific purposes. As part of the Computational Materials Sciences Network (CMSN) collaboration, the research group of Prof. Chelikowsky at the University of Minnesota is currently using our GW-BSE codes in a collaborative project. We are computing the optical properties of a series of diatomic molecules and comparing them to results from timedependent density functional theory (TDLDA). The findings show the limits of applicability of TDLDA and will hopefully point to new, improved energy functional within the density-functional formalism.

\section{Publications}

D. Raczkowski, A. Canning and L-W Wang, "The Grassman-metal All Band Iterative Diagonalization Method for Total Energy Calculations," Phys. Rev, B., submitted for publication, December 2002.

S. Istmail-Beigi and S.G. Louie, "Excited-state Forces within the Ab Initio Bethe-Salpeter Formalism," Phys. Rev. Lett., submitted for publication, August 2002.

S. Ismatil-Beigi C. Spataru and S.G. Louie, "Optical Properties of Carbon Nanotubes," Phys. Rev. B., in preparation.

\section{Investigation of Charge Transfer in Organic Electronics using Ultrafast Spectroscopy and Targeted Synthesis}

Principal Investigators: Charles Harris, John Arnold; John Kerr, and Stephen Johnson

Project No.: 02010

\section{Project Description}

This project is for the investigation and understanding of the dynamics and mechanisms of charge transfers at interfaces in organic electronic devices. Organic light emitting diodes (LEDs), organic photovoltaic cells, and organic electronic devices such as field-effect transistor (FETs), and memory are examples of such devices where there is much recent excitement, while more mature fields such as electrodeposition and lithium batteries continue to require fundamental understanding to realize their potential. In all of these applications, progress is hindered by a lack of fundamental understanding of the interfacial charge transport processes. It is the goal of this research proposal to focus a unique combination of capabilities upon this critical bottleneck area and to take leadership in the analysis and design of interfacial structures that lead to the development of robust and efficient, long-lived devices that will play a major role in meeting the nation's energy efficiency goals for the future.

Three major components of the research are envisioned:

- Application of ultra fast (femtosecond) spectroscopy techniques to organic-cathode interfaces for small molecule and polymer LEDs and to organic photovoltaic systems that utilize fullerenes, photo-sensitizers and conducting polymers. This phase will develop the full potential of the ultrafast spectral techniques to model interfaces relevant to practical devices.

- Synthesis of appropriate new molecules to facilitate the interfacial analyses and to provide relevant structure-function information.

- Construction and testing of devices that incorporate the new interfacial structures based on designs derived from the spectral analysis and structure-function relationships.

This approach not only diagnoses failure mechanisms but also leads to solutions that yield practical devices. 


\section{Accomplishments}

Ultrafast two-photon photoemission has been used to study electron solvation at two-dimensional metal/nitrile interfaces. The molecular motion that causes the excess electron solvation is manifested as a dynamic shift in the electronic energy. Although the initially excited electron is delocalized in the plane of the interface, interactions with the adsorbate can lead to its localization. A method for determining the spatial extent of the localized electron in the plane of the interface has been developed. The spatial extent of the electron was measured to be on the order of a single adsorbate molecule.

Time-resolved two-photon photoemission was also used to investigate the two-dimensional electron solvation by methanol, 1-propanol, 1-butanol, and 1-pentanol overlayers on a $\mathrm{Ag}(111)$ surface. For each system at coverages higher than one monolayer, several image potential state series with time-dependent energies were observed by using twophoton photoemission. This indicates multiple timedependent local workfunctions that can originate from simultaneous multiple coverages. The energy relaxation is attributed to the rotation of the adsorbate molecular dipoles to solvate the electron and is highly coverage dependent. This rotation lowers the electron-layer interaction energy, causing a dynamic reduction of the local workfunction. This represents the solvation of the electron by the adsorbate layer. A classical electron-disk dipole potential supports the conclusion that the dynamic variation in the innge potential state energies results from a local workfunction effect and indicates that the evolution of the energy results from the time-dependent projection of the molecular dipole onto the surface normal.

In addition, an effusion cell for controlled molecular beam epitaxial (MBE) growth of electroactive thin films in ultrahigh vacuum was constructed and used in preliminary experiments to understand the layer growth of trisquinolinolato aluminum onto a silver cathode. Further experimentation will examine the dynamics of electrons in these and other organic thin films.

\section{Publications}

A.D. Miller, I. Bezel, K.J. Gaffney, S. Garrett-Roe, S.H. Liu, P. Szymanski, C.B. Hartis, "Electron Solvation in Two Dimensions," Science, 297: 1163-1166 (2002).

S.H. Liu, A.D. Miller, K.J. Gaffney, P. Szymanski, S. Garrett-Roe, I. Bezel, C.B. Harris, "Direct Observation of Two-Dimensional Electron Solvation at an Alcohol/Ag(111) Interface," J. Phys. Chem. B, 106: 1290812915 (2002) 



\section{Acronyms and Abbreviations}

\begin{tabular}{|c|c|c|c|}
\hline $\begin{array}{l}\text { ADC } \\
\text { AFM }\end{array}$ & $\begin{array}{l}\text { analog to digital converter } \\
\text { atomic force microscopy }\end{array}$ & FIIA & $\begin{array}{l}\text { Fourier transform infrared } \\
\text { spectroscopy }\end{array}$ \\
\hline AFRD & $\begin{array}{l}\text { Accelerator and Fusion Research } \\
\text { Division }\end{array}$ & $\begin{array}{l}\text { FY } \\
\text { GFP }\end{array}$ & $\begin{array}{l}\text { fiscal year } \\
\text { green fluorescent protein }\end{array}$ \\
\hline ALS & Advanced Light Source & GGA & generalized gradient \\
\hline ANL & Argonne National Laboratory & & approximation \\
\hline $\begin{array}{l}\text { APC } \\
\text { ARPES }\end{array}$ & $\begin{array}{l}\text { antigen presenting cell } \\
\text { angle resolved photoemission }\end{array}$ & GRETA & $\begin{array}{l}\text { Gamma-Ray Energy Tracking } \\
\text { Array }\end{array}$ \\
\hline & spectroscopy & HDL & high density lipoprotein \\
\hline BNL & Brookhaven National Laboratory & HEP & High Energy Physics \\
\hline $\begin{array}{l}\text { BNCT } \\
\text { BOOMERANG }\end{array}$ & $\begin{array}{l}\text { Boron Neutron Capture Therapy } \\
\text { Balloon Observations of }\end{array}$ & HPPES & $\begin{array}{l}\text { High-pressure photoelectron } \\
\text { spectroscopy }\end{array}$ \\
\hline & $\begin{array}{l}\text { Millimetric Extragalactic Radiation } \\
\text { and Geophysics }\end{array}$ & $\begin{array}{l}\text { HPSS } \\
\text { HRV }\end{array}$ & $\begin{array}{l}\text { high performance storage system } \\
\text { human rhinovirus }\end{array}$ \\
\hline CCD & charge-coupled device & IR & infrared \\
\hline CERN & $\begin{array}{l}\text { the European Laboratory for } \\
\text { Particle Physics near Geneva, } \\
\text { Switzerland }\end{array}$ & $\begin{array}{l}\text { IRAM } \\
\text { JGI } \\
\text { JPL }\end{array}$ & $\begin{array}{l}\text { intelligent RAM } \\
\text { Joint Genome Institute } \\
\text { Jet Propulsion Laboratory }\end{array}$ \\
\hline $\begin{array}{l}\mathrm{CH} \\
\mathrm{CHD}\end{array}$ & $\begin{array}{l}\text { Chemical Sciences Division } \\
\text { coronary heart disease }\end{array}$ & P'OASIS & $\begin{array}{l}\text { laser Optics and Acceleration } \\
\text { Systems Integrated Studies }\end{array}$ \\
\hline CMB & cosmic microwave background & LBNL & Ernest Orlando Lawrence \\
\hline CMOS & $\begin{array}{l}\text { Complementary metal-oxide } \\
\text { semiconductor }\end{array}$ & LDL & $\begin{array}{l}\text { Berkeley National Laboratory } \\
\text { low density lipoprotein }\end{array}$ \\
\hline CMR & colossal magnetoresistive & LDA & local density approximation \\
\hline $\begin{array}{l}\mathrm{COG} \\
\mathrm{CPU}\end{array}$ & $\begin{array}{l}\text { clusters of orthologous groups } \\
\text { central processing unit }\end{array}$ & LDAD & $\begin{array}{l}\text { Laboratory Directed Research and } \\
\text { Development }\end{array}$ \\
\hline CQW & coupled quantum well & LEDs & light emitting diodes \\
\hline $\operatorname{CS}$ & Computing Sciences & $\mathrm{LHC}$ & Large Hadroni Collider, CERN \\
\hline $\mathrm{cW}$ & continuous wave & LP & lipoprotein \\
\hline DFT & density functional theory & LSD & Life Sciences Division \\
\hline DNA & deoxyribonucleic acid & MAXIMA & Millimeter Anisotropy eXperiment \\
\hline DOD & Department of Defense & & Imaging Array \\
\hline $\begin{array}{l}\text { DOE } \\
\text { DRAM }\end{array}$ & $\begin{array}{l}\text { Department of Energy } \\
\text { dynamic random access memory }\end{array}$ & MCF & $\begin{array}{l}\text { Macromolecular Crystallography } \\
\text { Facility, ALS }\end{array}$ \\
\hline ED & Engineering Division & MES & molecular environmental science \\
\hline EDS & Energy dispersion spectroscopy & MPI & message passing interface \\
\hline EETD & $\begin{array}{l}\text { Environmental Energy } \\
\text { Technologies Division }\end{array}$ & $\begin{array}{l}\text { MSD } \\
\text { NASA }\end{array}$ & $\begin{array}{l}\text { Materials Sciences Division } \\
\text { National Aeronautics and Space }\end{array}$ \\
\hline EM & electromagnetic & & Administration \\
\hline FNAL & $\begin{array}{l}\text { Fermi National Accelerator } \\
\text { Laboratory }\end{array}$ & NCAR & $\begin{array}{l}\text { National Center for Atmospheric } \\
\text { Research }\end{array}$ \\
\hline EPA & Environmental Protection Agency & NEMS & nanoelectromechanical systems \\
\hline ESA & European Space Agency & NERSC & National Energy Research \\
\hline ESD & Earth Sciences Division & & Scientific Computing \\
\hline $\begin{array}{l}\text { ESDG } \\
\text { ETS }\end{array}$ & $\begin{array}{l}\text { Earth Science Data Grid } \\
\text { environmental tobacco smoke }\end{array}$ & NEXAFS & $\begin{array}{l}\text { near-edge } x \text {-ray adsorption fine } \\
\text { structure }\end{array}$ \\
\hline EXAFS & $\begin{array}{l}\text { extended } x \text {-ray absorption fine } \\
\text { structure }\end{array}$ & $\begin{array}{l}\mathrm{NIH} \\
\mathrm{NMR}\end{array}$ & $\begin{array}{l}\text { National Institutes of Health } \\
\text { nuclear magnetic resonance }\end{array}$ \\
\hline $\begin{array}{l}\text { FHWM } \\
\text { FETs }\end{array}$ & $\begin{array}{l}\text { full width at half maximum } \\
\text { field-effect transistor }\end{array}$ & NOAA & $\begin{array}{l}\text { National Oceanic Atmospheric } \\
\text { Administration }\end{array}$ \\
\hline $\mathrm{FISH}$ & Fluorescence in situ hybridization & NSD & Nuclear Science Division \\
\hline $\mathrm{AF}$ & fluorescence-resonance-energy & NSF & National Science Foundation \\
\hline fs & $\begin{array}{l}\text { transfer } \\
\text { femtosecond }\end{array}$ & $\begin{array}{l}\text { PBD } \\
\text { PCB }\end{array}$ & $\begin{array}{l}\text { Physical Biosciences Division } \\
\text { printed circuit boards }\end{array}$ \\
\hline
\end{tabular}




\begin{tabular}{|c|c|c|c|}
\hline PCR & polymerase chain reaction & SNAP & SuperNova/Acceleration Probe \\
\hline PD & Physics Division & SNO & Sudbury Neutrino Observatory \\
\hline $\begin{array}{l}\text { PDE } \\
\text { PDSF }\end{array}$ & $\begin{array}{l}\text { Partial Differential Equation } \\
\text { Parallel Distributed Systems }\end{array}$ & SQUID & $\begin{array}{l}\text { Superconducting QUantum } \\
\text { Interference Device }\end{array}$ \\
\hline & Facility & SSAL & Stanford Synchrotron Radiation \\
\hline PEEM & Photo-Electron Emission & & Laboratory \\
\hline & Microscope & STAR & Solenoidal Tracker at RHIC \\
\hline $\begin{array}{l}\text { PET } \\
\text { PI }\end{array}$ & $\begin{array}{l}\text { positron emission tomography } \\
\text { principal investigator }\end{array}$ & STM & $\begin{array}{l}\text { scanning tunneling microscope or } \\
\text { microscopy }\end{array}$ \\
\hline PIM & processor-in-memory & SVD & singular value decomposition \\
\hline & Particulate matter & SXH & soft $x$-ray holography \\
\hline POLARBEAR & $\begin{array}{l}\text { POLARization of the Background } \\
\text { millimEter background Radiation }\end{array}$ & $\begin{array}{l}\text { SXRD } \\
\text { SXRF }\end{array}$ & $\begin{array}{l}\text { scanning } x \text {-ray diffraction } \\
\text { synchrotron } x \text {-ray fluorescence }\end{array}$ \\
\hline $\begin{array}{l}\text { PPDG } \\
\text { QW }\end{array}$ & $\begin{array}{l}\text { Particle Physics Data Grid } \\
\text { Quantum Well }\end{array}$ & SXTM & $\begin{array}{l}\text { scanning transmission } x \text {-ray } \\
\text { microscope }\end{array}$ \\
\hline $\mathrm{sf}$ & radio frequency & TCR & T-cell receptors \\
\hline RHEED & $\begin{array}{l}\text { reflection high energy electron } \\
\text { diffraction }\end{array}$ & $\begin{array}{l}\text { TEM } \\
\text { TES }\end{array}$ & $\begin{array}{l}\text { Transmission Electron Microscope } \\
\text { transition-edge sensors }\end{array}$ \\
\hline $\mathrm{RHIC}$ & $\begin{array}{l}\text { Relativistic Heavy Ion Collider, } \\
\text { Brookhaven National Laboratory }\end{array}$ & $\begin{array}{l}\text { TRNA } \\
\text { UC }\end{array}$ & $\begin{array}{l}\text { transfer RNA } \\
\text { University of California }\end{array}$ \\
\hline RNA & ribonucleic acid & UHV & ultra-high vacuum \\
\hline SAXS & small-angle $x$-ray scattering & UV & ultraviolet \\
\hline & Office of Science, DOE & VLDL & very low density lipoprotein \\
\hline SciDac & $\begin{array}{l}\text { Scientific Discovery through } \\
\text { Advanced Computing }\end{array}$ & $\begin{array}{l}\text { VOC } \\
\text { VSOM }\end{array}$ & $\begin{array}{l}\text { volatile organic compound } \\
\text { visual servoing optical microscopy }\end{array}$ \\
\hline SEM & scanning electron microscope & WAXS & wide-angle $x$-ray scattering \\
\hline SF-FTIR & $\begin{array}{l}\text { Synchrotron Radiation Fourier } \\
\text { Transform Infrared }\end{array}$ & $\begin{array}{l}\text { XAFS } \\
\text { XANES }\end{array}$ & $\begin{array}{l}\text { x-ray absorption fine structure } \\
\text { x-ray absorption near-edge }\end{array}$ \\
\hline AC & Stanford Linear Accelerator & & structure spectroscopy \\
\hline & Center & XAS & $x$-ray absorption spectroscopy \\
\hline SLIM & $\begin{array}{l}\text { structured light-imaging } \\
\text { microscope }\end{array}$ & $\begin{array}{l}\text { XFH } \\
\text { XRD }\end{array}$ & $\begin{array}{l}x \text {-ray fluorescence holography } \\
\text { x-ray diffraction }\end{array}$ \\
\hline
\end{tabular}

